

\section{Design Transactions}

Rethinking Information Modelling for a New Material Age

UCLPRESS B 


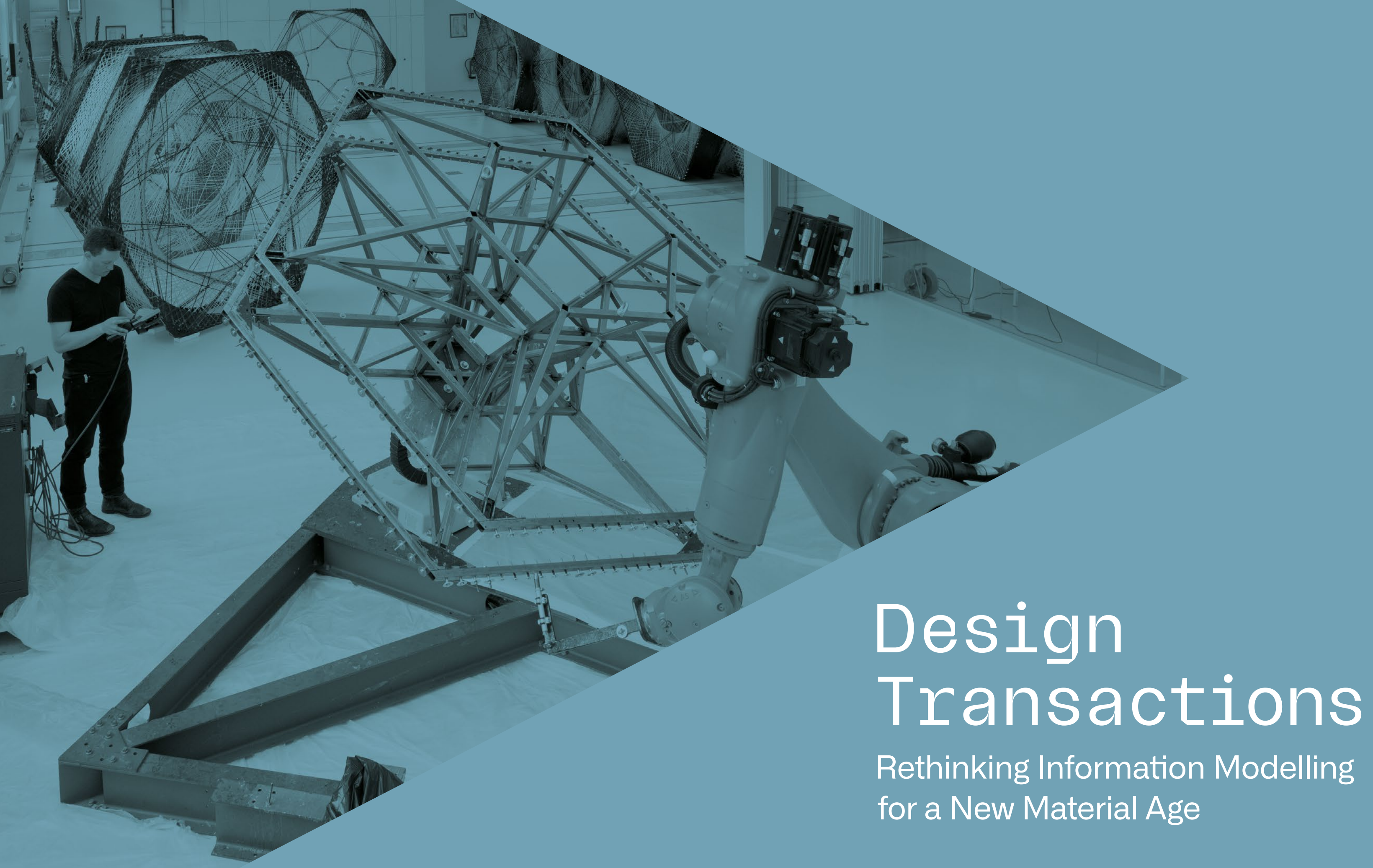




\section{Contents}

\section{INTRODUCTIONS}

$8 \quad 11$

Design Transaction

1.2

Thensen and Martin Tamke

Innochain: A Template for Innovative

Collaboration

an Knippers, Bob Sheil and

Mette Ramsgaard Thomsen

221.3

Design Methodologies: Rethinking Transaction Between the Drawn and the Made

Bob Sheil, Mathilde Marengo and Ulrika Karlsson

301.

Perspectives: Transactions and Trajectories Bob Sheil and Mette Ramsgaard Thomsen

MODELLING STRATEGIES

ANDNEWWORKFLOWS

$42 \quad 2.1$

New Paradigms for Digital Prefabrication in Architecture

Fabian Scheurer and Hanno Stehling

$50 \quad 2.2$

Information-Rich Exploration

in the Early Design Phase

Zeynep Aksöz

$56 \quad 2.3$

Computational Extensibility and Mass Participation in Design

Paul Poinet and Al Fisher

$62 \quad 2.4$

Alternative Means of Digital Design Communication

Dimitrie Stefanescu

$68 \quad 2.5$

Negotiating Structured Buildin Information Data

Nathan Miller and David Stasiuk
74 Research Summary

Design Exploration

in the Early Design Phase

Zeynep Aksöz

76 Research Summary

Morphogenetic Fluid Dynamics

Angelos Chronis

78 Research Summary

Multiple States of Equilibrium for

Bending-Active Tensile Structures

Evy L. M. Slabbinck

80 Research Summary

Alternative Methods for Data Transaction

Dimitrie Stefanescu

82 Research Summary

Integrated Material Practice in Freeform

Timber Structures

Tom Svilans

84 Further Perspectives

New Workflows and Collaborations

Kenn Clausen, Sean Lineham, Morten Norman

Lund, Line Rahbek and Kåre Stokholm

Poulsgaard

DESIGN INTEGRATION

$92 \quad 3.1$

Design Integration: Global Technologica

Advancement and Local Culture

In dialogue: Areti Markopoulou and Philip F. Yuan

$98 \quad 32$

Agency of Material Production Feedback

in Architectural Practice

Tom Svilans, Jonas Runberger and Kai Strehlke

$106 \quad 3.3$

Acoustic Wall: Computational and Robotic

Design Integration of Four Primary Generators

Isak Worre Foged, Anke Pasold and

Mads Brath Jensen

$114 \quad 3.4$

Coreless Filament Winding: From Academia to Practice

James Solly, Jan Knippers and Moritz Dörstelmann
$122 \quad 3.5$

All That Is Porous: Practising

Cross-Disciplinary Design Thinking

Vasily Sitnikov

128 Research Summary

Bend\&Block: A Passive Form-Giving Strate

Efilena Baseta

130 Research Summary

Adaptive Robotic Carving

Giulio Brugnaro

132 Research Summary

How to Effectively Collaborate at Scale

in a Connected Digital Environment

Paul Poinet

134 Research Summary

Ice Formwork: Challenging the Sustainable Production of Non-Regular Geometry in Concrete

136 Research Summary

Virtual Prototyping Tools for a Winding-Based Composite Fabrication Technique

James Solly

138 Further Perspectives

Data in Design Practice

Sean Hanna

NOVEL STRATEGIES FOR MATERIALISATION

$144 \quad 4.1$

Rewired Engineering: The Impact of

Customisation and Interoperability on Design

Edoardo Tibuzzi

$150 \quad 4.2$

Drone Spraying on Light Formwork for Mud Shells Stephanie Chaltiel, Maite Bravo,

Diederik Veenendaal and Gavin Sayers

$158 \quad 4.3$

Industrialising Concrete 3D Printing:

Three Case Studies

Nadja Gaudillière, Justin Dirrenberger,

Romain Duballet, Charles Bouyssou,

Alban Mallet, Philippe Roux and Mahriz Zaken
$66 \quad 4$

Integration of Material and Fabrication

Affordances within the Design Workflow

Giulio Brugnaro, Silvan Oesterle, Sean Hanna,

$174 \quad 4.5$ Peter Scully and Bob Sheil

Making Timberdome

$180 \quad 4.6$

作

Robots for Skill Digitisation

Johannes Braumann

188 Research Summary

Building with Earth and Drones

Stephanie Chaltiel

190 Research Summary

Information Modelling for Assembly Planning, Sequencing and Optimisation

Ayoub Lharchi

192 Research Summary

Digital Clay: Hybrid Manufacturing

Arthur Prior

194 Research Summary

Flectofold: From Academic Research

to Architectural Application

Saman Saffarian

196 Research Summary

Concrete Deposition: Choreographing Flow

198 Further Perspectives

Performative Materials and Systems,

and Additive Manufacturing Futures

Johannes Braumann and Isak Worre Foged

REFLECTIONS

$206 \quad 5.1$

Innochain: External Perspectives

Mark Burry and Christoph Gengnage

Mark Burry and Christoph Gengnage

212 BIOGRAPHIES

23 ACKNOWLEDGEMENTS

224 COLOPHON 


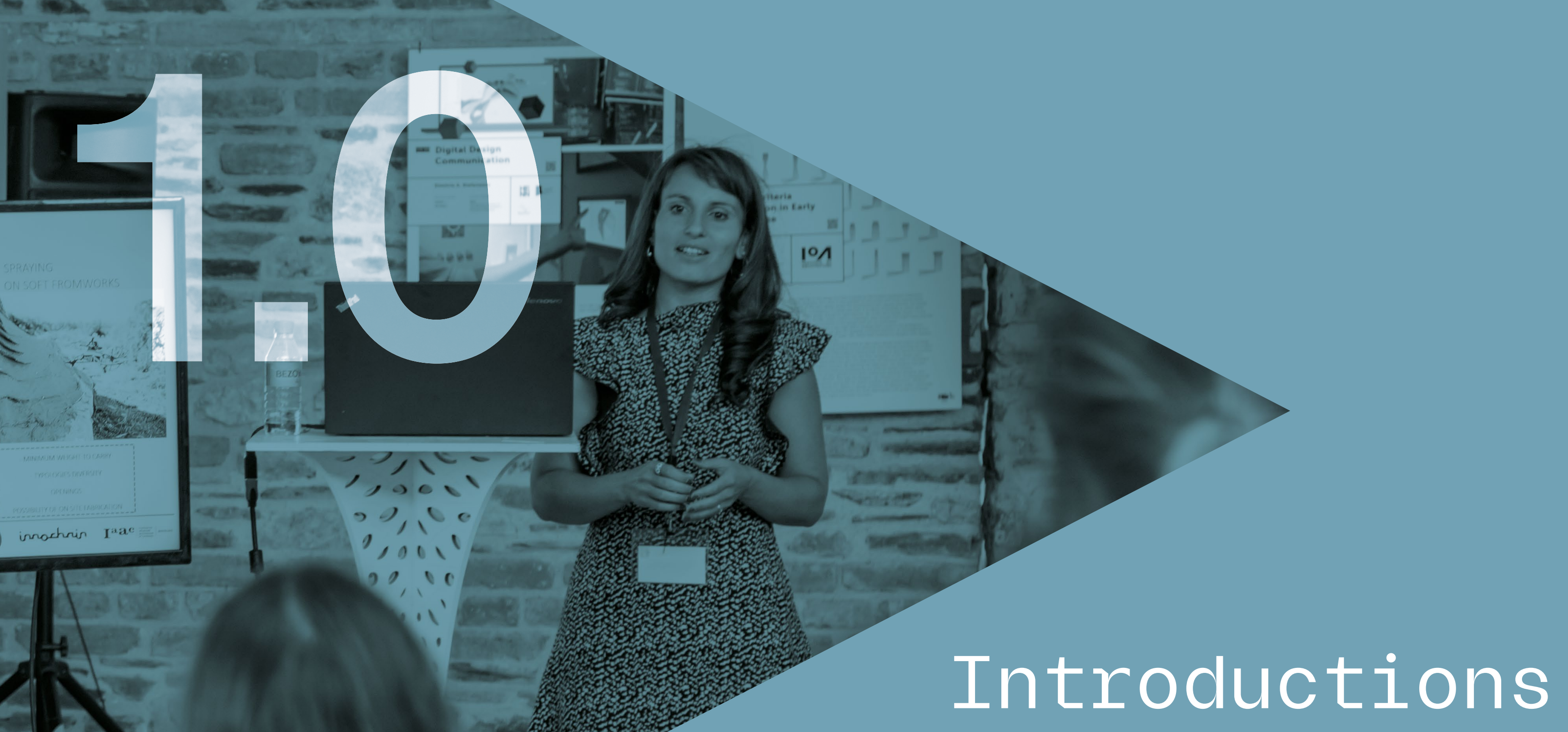




\section{Design Transactions}

Mette Ramsgaard Thomsen and Martin Tamke

Centre for Information Technology and Architecture (CITA), the Royal Danish Academy of Fine Arts, Schools of Architecture, Design and Conservation
Design Transactions asks what the future of building culture will be. It asks how new, shared computational platforms are changing our disciplines, examining how the digitisation of tools affects the way architecture is conceived designed and made. Questions arise as we enter a new era of advanced modelling, informed by new concepts of Big Data computing, cloud-based collaboration and steered robotic fabrication: What might collaboration look like in the future? How can knowledge across the design change be interfaced and fed back for a more informed and materially-sensitive practice? What is the future for automation in architecture?

Today, computational design is ubiquitous in buildin practice; the tools of design, analysis, specification and manufacture are now primarily digital. While tools vary in sophistication and programmability they share

a common disital foundation. This makes them

fundamentally open to interfacing which in turn,

has led to the conception of a digital chain via which

information is communicated, connected and extended inform vision has framed building practice for the last 15 years vision has framed building practic

Yet, despite this, the building industry remains unable to reap the benefits of technological progress. Practice remains fractured and issues of ownership, disciplinebased silo-thinking and legal propretary boundaries continue to obstuct meaningul sharing and innovation. This paralys has significant consequences inovation. the inality to sect the un consequences, including of building entect the urgently-needed restructuring loss of proutive, which has contributed to a profound famously, one of the least efficient industries, having hit a plateau in production growth over the last 20 years. here construction-related spending accounts for $13 \%$
1\& 2. Practice Futures - Building Design for a new Ma Fulalage by showcasing reserch by the programme's Smedie Exhibition Hall, KADK, 2018 .

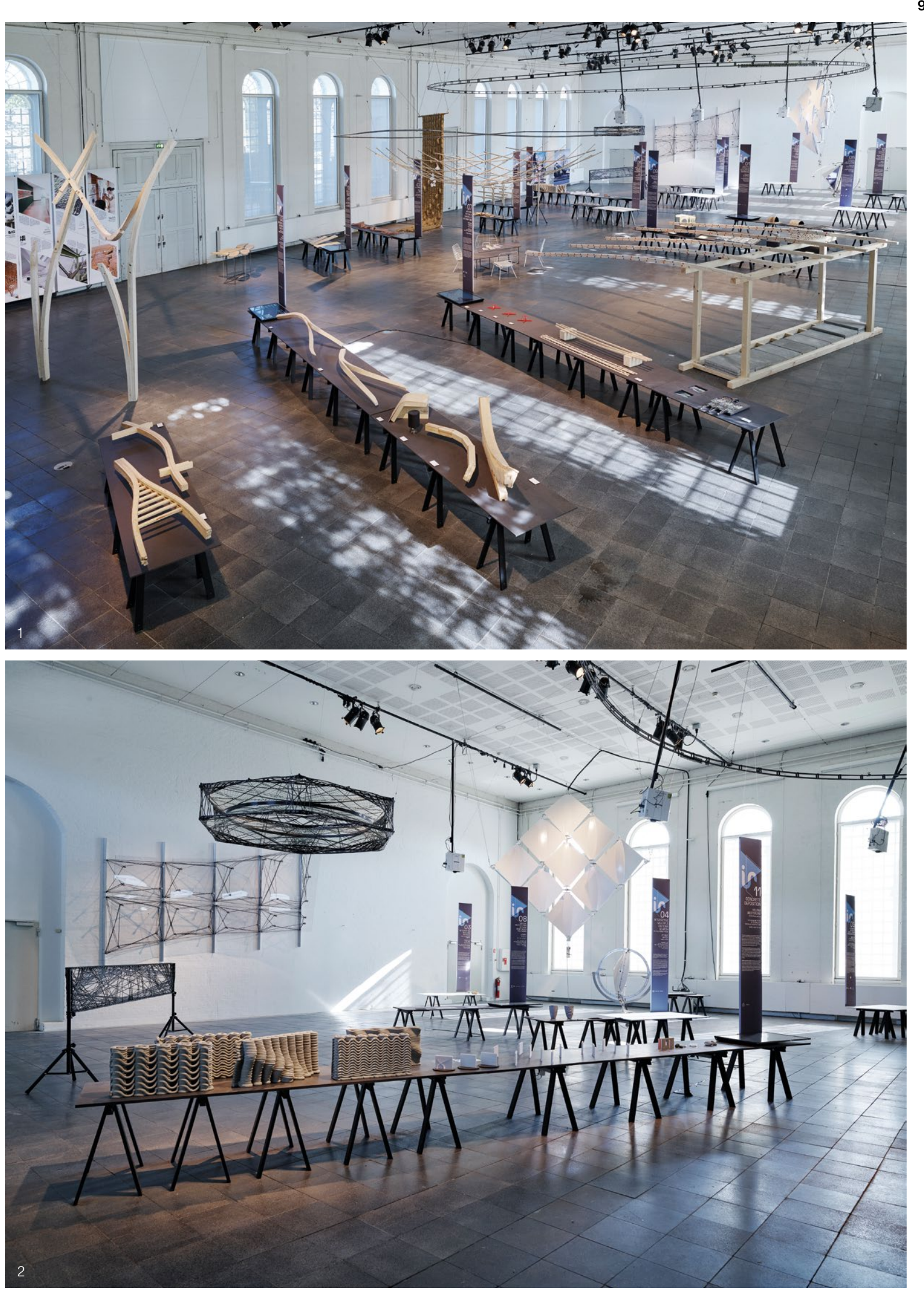




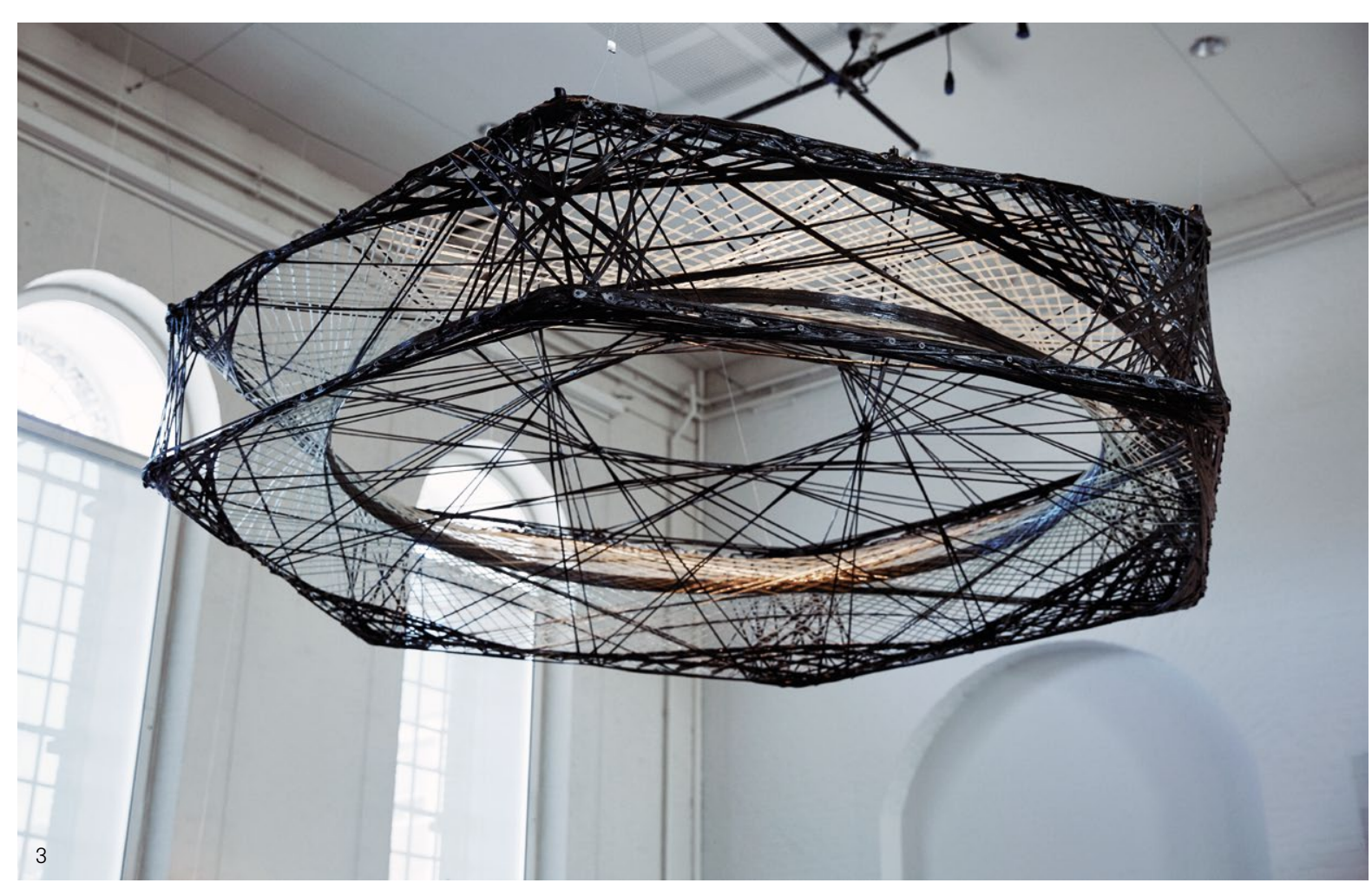

of the world's GDP, the sector's annual growth has

increased by only $1 \%$ over the past 20 years (Barbosa et al., 2017). As we encounter new contexts

of environmental crisis, resource scarcity and climate

change, these consequences expand beyond the

economic realm and into the ethical. It is therefore

imperative that we find our way across the constraints

that limit the potential of the digital chain, to deepen

its positive impact and extend its disciplinary reach.

The urgent call for innovation is trifold: to identify new

methods to optimise our building practices and new

us to rethink the resources, energy consumption and

climate adaptation of our buildings: to challenge the way

climate adaptation of our bulldings, to challenge the way

and to profoundly rethink the techno

and to profoundy rethich

moving us from
additive ones.

Computational design is continually evolving, and new tools present new opportunities. Digitisation and the creation of new methods to optimise construction processes and material performance remain key to benefitting from the innovation taking place (Ramsgaard foomsen, 2019). The first generation of computation focused on developing shared industry standards, such as the Bullding Information Modelling (BIM) paradigm and the IFC standards, as well as maturing design-tofabrication protocols that enabled individualised and mass-customised fabrication (Klinger, 2008). Knowledge
transfer across the fields of Big Data, advanced sensing

3. Prototype Canopy Component. Elytra
Filament Pavilion. Exhibited by James Solly, ITKE. Photo: Anders Ingvartsen. and robotic steering is challenging how we understand these models. Contemporary practice is reassessing what kind of information the model should contain, how it is shared and how it interfaces and back-propagates or 'handshakes' with other models, thus refining and reshaping the digital chain.

This fundamental re-examination of what advanced computation affords is also apparent in the continuous rethinking of fabrication. If the first generation of digitisation mainly focused on known material systems, contextualised by existing industrial processes, the a new generation of computational design research understands fabrication as a means of challenging the material practices of bullding cultures. The interfacing of host of new and old materials, such as high-performance fibres (Ramsgaard Thomsen, 2015; Menges, 2015), me plates (Nicholas, 2016), concrete (Sitnikov, 2014) and earthen materials (Samec, et al., 2019), presents new alternatives to how we understand building systems.

\section{Design Perspectives}

Design Transactions captures the cutting edge of these innovations. Connecting research in academia and in practice, the book presents case studies examining the future methodologies and underlying paradigms of an information-rich design practice.

The projects showcased here challenge our thinking of how the practices of design, analysis and fabrication can intersect, and the perspective is purposefully wide. By engaging the breadth of the design chain and its disciplines, we ask how opportunities for advanced computation, integrated simulation and performance evaluation, human-computer interaction and robotic fabrication can create tangible differences in the way we build. What is at stake here is, on the one hand the future of the design model and the collo one hand, the of building practice, and on the other, the way we understand our fabrication culture and the meterial systems with which we build By exponding the simple systems with which we buld. By expanding the simple engape and capitise on analysis, to protype - to engage and capith morecomplex requirements - to cloud-based com ro automation and robotic fabrication - we explore the datarich design data rich design inquires, en ble collon

\section{Foundations}

Design Transactions takes its point of departure from the cross-disciplinary research network Innochain. the cross-disciplinary research network Innochain. Innochain is a European Union Horizon 2020 Innovation Training Network that brings together six internationally recognised academic research facilities (with a focus on and 14 pioneering industry partners from architecture engineering, design software development and generation of interdisciplinary practitioners with specia inter-sector knowledge that traverses academia and industry on the specifics of the expanded digital design chain. With a focus on new computational design strategies, advanced design-integrated analysis and robotic fabrication systems, Innochain has established 15 new research collaborations across multiple disciplines and practices. The research horizon of these projects is intentionally wide, spanning from the applied and industry-ready context to the speculative. All projects have a foothold in academia and engage with different industry partners, creating novel relationships across the value chain. Some employ industry-based case studies for research investigation; some use industry partners' design information as empirical data; and others retain a speculative stance, asking 'blue sky' questions on how practices might change when

engaging new technologies.

As a result, the work produced via the Innochain network is extremely diverse. The projects ask what new computational methods there are, from the examination of design interfaces for interdisciplinary collaboration to open cloud-based communication platforms, and nove means of integrating computational fluid dynamics (CFD) simulation to the Big Data strategies of machine learning for design investigation. The work presented here also explores the materialisation of architecture developing novel ways of casting concrete with ice formwork, large-scale additive manufacture with concrete, clay, and drone-spraying mud structures. From their respective vantage points, the authors ask how advanced computational technologies can enable sustainable informed and materially-smart design solutions.

What the projects have in common is an experimental design-led research method focused on the development of either prototypical applications - demonstrating new computational paradigms - or full-scale materia prototypes testing

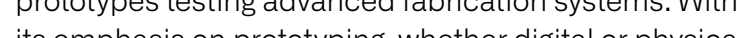
Innochain engages directly with the investigated methods and technocies, positioning the rel methods and technologles, positioning the research inquires with a network of

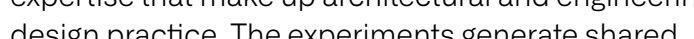
design practice. The experinents generate shared empirical data that can be tested, analysed and evaluated by the different research teams across the network, producing results that, in turn, can industrial partnership. 
12

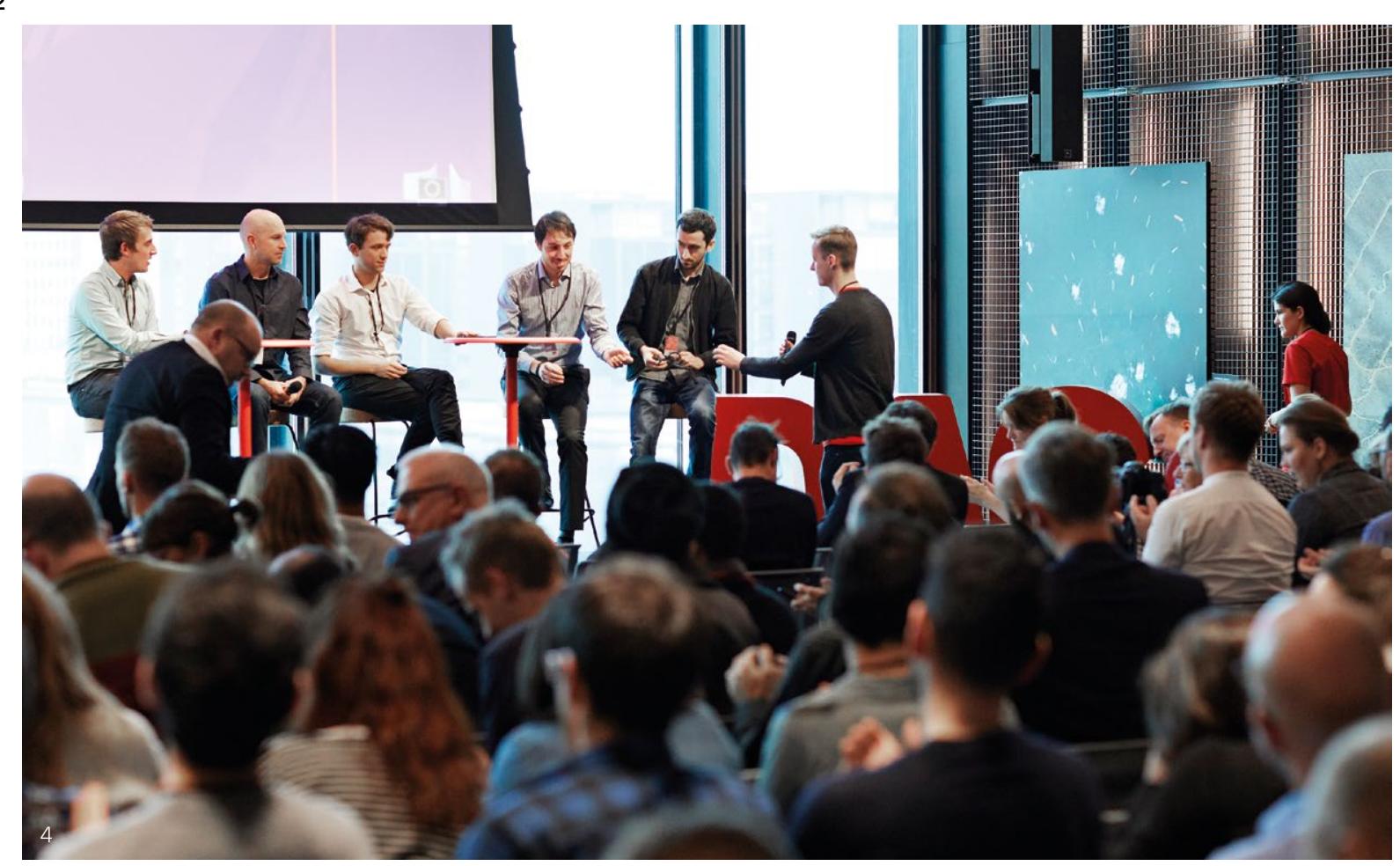

Expanding Information Modelling

Design Transactions shares some of the primary research findings of Innochain, while presenting the wider context of these inquiries. It also brings together the most prolific keynote presentations and peerreviewed papers from the Innochain conference 'Expanding Information Modelling' (November 2018, Danish Architecture Centre, Copenhagen). With three Danish Architecture Centre, Copenhagen). With the
core themes providing the structure for the book 'Advanced Modelling Strategies and New Wook 'Advanced Modelling Strategies and New Workflows', 'Design Integration' and 'Material Strategies' - the key 'Advanced Modelling Strategies and New Workflows' Advanced Moch his thateges and New Work Hor collects case studies that focus on the analysis,
synthesis and communication of data in design th synthesis and communication of data in design think be established andintegrated or th design practice. "Design Integration' focuses on the emergence of hyb practices in the bulding industry and discusses how this digtal chain can be structured, how collaboration between partners at different ends of the design chain can be productive and how early design thinking with structural and material information can lead to smarter design solutions. Material Strategies, meanwhile, questions the potential of computer-controlled design-to-fabrication strategies to effect efficient, environmentally safe and sustainable building practices. The chapter discusses how these new work Hows can be structured and how they present a rethinking of the relationship between man and machine, radically questioning established processes.

Each chapter is framed by an introductory keynote piece. Here, reflections are brought together to explore design futures. Fabian Scheurer and Hanno Stehling of Design-to-Production discuss the design and reflect on their work on complex-form solid timberframe buildings and how feedback between the design phase and fabrication led to new design opportunities. Philip $F$. and fabrication led to new design opportunities. Philip F. principal of Archi-Union, and Areti Markopoulou, Academic Director at the Institute for Advanced computationally-steered design and mas how

computationally steered design and manufacturing crafts. And Edoard Tibuzzi from AKTI ceramines how (d) computational design is engineering practices. practice and project presentations, ranging from consolidated large-scale design offices with dedicated research groups to comparatively young, small-scale With a strong collaborative perspective, these With a strong collaborative perspective, these presentations ask profound questions regarding how we understand collaboration, what infrastructures are needed to support real design feedback and how formalised material understanding can support robotic
fabrication systems. manufacture of the Headquarters for Swatch. They

Yuan, Professor of Tongji University in Shanghai and

The keynos are followed by contributions from

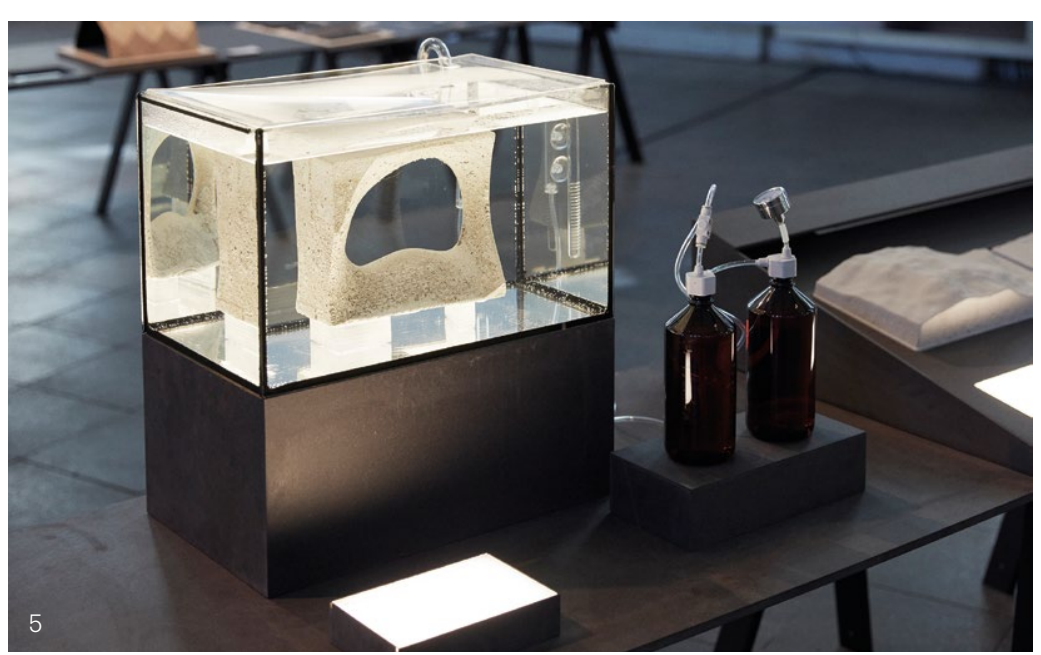

References

Barbosa, F., Woetzel, J., Mischke, J.,
Ribeirinho, M.J., Sridhar, M., Parsons, M. Be tram, N. and Brown, S. 2017, Reinventing Washington DC, McKinsey Global Institute.

Klinger, K.R., 2008, 'Relations: Information Architecture' in Kolarevic, B. and Klinger, $K$ (eds.), 2016, Manufacturing Material Effects.
Rethinking Design and Making in Rethinking Design and Making in
Architecture, London, Routledge, $.25-36$ Kolarevic, B., 2003, 'Digital Morphogenesis' in Architecture in the Digital Age: Design
and Manufacture, London, Spon Press, .13. Menges, A. and Knippers, J., 2015, ‘Fibrous
Tectonics' in Architectural Design, Vol. 85 , Tectonics' in Ar
No. 5, p.40-47.

Conclusion

Design Transactions is intended to offer readers a sense of the future direction of computational design across building practice, with case studies of new collaborative practice enabled by computational advances that radically rethinks what the digital chain can be. While the array of examples presented here remain prototypical, they are actively building the kind of foundational methods and technologies that are needed for a sectorwide change, reshaping how partnerships within building practice can work together.

This volume has been made possible thanks to the many contributions from the various networks it brings together. We are thankful for the collaborative effort and generous knowledge-sharing of all the contributors, as well as the invaluable input of the wider Innochain as well as the invaluable input of the wider Innochain

network. These include industry partners: Foster + Partners; White; BIG; Henn; ROK; Cloud 9; BuroHap
str.ucture; Design-to-Production: Smith Innovation: str.ucture; Design-to-Production; Smith Innovation; and FibR; as well as the associated partnership Robots. and FibR, as wal Bartlt Scho actic part Bartetts the institute of Architecture (IOA) School of Stutgar, the Instilute of Archilecture (IOA), School for the facture $K$, for the facllation provided by. We Horzon 2020 Marie Skłodowska-Cuneactions, togetherwith various industry partnerships and institutions, all of which have helped BLOXHUB Science Forum for its collabothe thank BLOXHUB Science Forum
Nicholas, P.,Zwierzycki, M., Stasiuk, D., Nergaard, E.C., Leinweber, S. and
Ramsgaard Thomsen, M., 2016, 'Adaptive Meshing for Bi-directional Information Flows: A Multi-Scale Approach to Integrating Feedback between Design
Simulation, and Fabrication' Adriaenssens, S., Gramazio, F., Kohler, M., Menges, A. and Pauly, M. (eds.), Advances vdf Hochschulverlag AG an der ETHZ Zürich, p.260-273.

Ramsgaard Thomsen, M., 2019, "Radical Cross-Disciplinarity: Laying the
Foundations for New Matrib Lratices in Construction Robotics. https://doi.

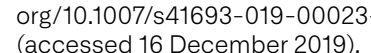

Ramsgaard Thomsen, M., Tamke, M., H.L., Gengnagel, C., and Schmeck, M. 2015, 'Hybrid Tower, Designing Soft Structures' in Thomsen, M.R., Tamke, M., Gengnagel, Faircloth, B. and Scheurer, F. (eds.),
Modelling Behaviour: Design Modelling
Symposium 2015, Berlin, Springer, p.87-99. Šamec, E., Srivastava, A. and Chaltiel, S. Monolithic Shells' in Proceedings of the International Conference on Sustainable (SMSS2019): Challenges in Design and Management of Structures (conference paper), Rovinj, Croatia.

Sitnikov, V., 2014, 'Monolith Translucent
Lattice' in Proceedings of ACADIA 14: Design Agency, Projects of the 34th Computer Aided Desirn An Archiation for Los Angeles, p.263-264.

4. Innochain conference 'Expanding
Information Modelling', Danish Architecture Centre, 2018. Photo: Philip Ørneborg. 5. Ice Formwork de-moulding process
demonstrator, Vasily Sitnikov KTT. Photo: Anders Ingvertsen 


\section{Innochain: A Template for Innovative Collaboration}

\section{Jan Knippers}

Puilding Structures and Structural Design (ITKE) at the University of Stuttgart Bob Sheil

The Bartlett School of Architecture, UCL

Mette Ramsgaard Thomsen

Centre for Information Technology and Architecture (CITA), the Royal Danish Academy of Fine Arts,

Schools of Architecture, Design and Conservation

Education, research and practice have evolved cyclically within the disciplines of architecture and engineering.

Visions are in continual dialogue with challenges,

experiments with experiences, plans with projects,

prototypes with constructs, and theories with

practicalities. Connecting each of these cycles is

a constellation of nodes and flows, with centres of

excellence and concentrations of activity. Intellectual

and physical mobility across this matrix is oscillating

and the resulting consequences are played out in the

perpetual adaptation of our built environment - in cities,

villages, ports and infrastructures - every minute, every

day, in actuality and simulation. Here, the scene is set for

how conventional research exchange between academia

and industry has been challenged by the Innochain

project. We depict the fundamental role that the

seamless relationship between new forms of practice,

innovative academic laboratories and pioneering new

industries plays in tackling the built environment's

greatest challenges.

Innochain: Building Innovation

in the Extended Digital Chain

Starting in late 2015, Innochain established a unique international research environment examining how advances in disital design tools challange building culture, enabling more sustainable, informed and materially-smert design solutions. As a network six academic institutions, 14 industry partwers ar 15 early-stere rear training proserame focused on the supervision of individual research projects, an inter-sector secondment programme and collective research events including

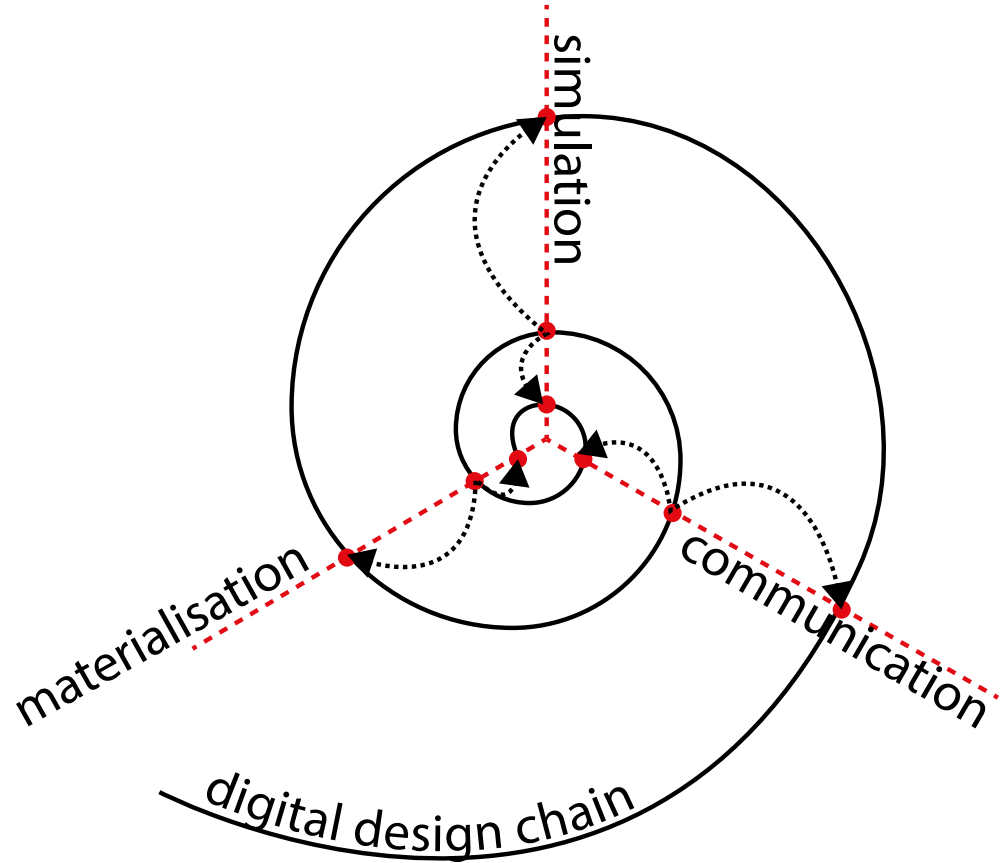

search courses for the ESRs to share knowledge and learn new skills. With each researcher positioned between strong innovative research practice and influential industrial impact, a new generation of interdisciplinary researchers has emerged, effecting change in the way that we consider, design and build environment.

Innochain's philosophy can be understood as a field of an infinite spiral intersected by lines of concentration that cut square to the spiral field generating recurrent tersections that revolve and evove knowledge and its

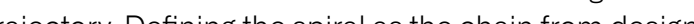
trajectory. Defin axes of research enquiry and disuption that underpin its axes of reseach innachan identied the intersecting ciscion. Com this bas, the se axes and spials have chim. On this basis, these axes and spirals have him la holistic and integrated design method hat interface

\section{A Matter of Urgency}

The scientifically-informed goals of the United Nations Framework Convention on Climate Change deman the wrastically reduce the consumption of finite
- especially fossil - resources for building construction and operations and thus fundamentally rethink the way we design and build architecture. Current rates of world population growth require that we build approximately the same living space over the next 30 years as we have to date, which will require a doubling of the entire building stock that currently exists. To do this, we must significantly increase the productivity of our construction processes and devise new methods and processes that reconcile

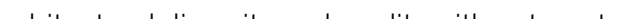
prefabrication, willy not repeating the falling of past attempts at prefabrication from 50 or 60 years ag.

It is a pardaxicalchallenge to buld twice as much

is a paradoxicalch fromge to buld twice as much cleaner tha ever before and in less tin a if this is to be cleaner the us er beferis an to be building systems must be optimised in a parlel and

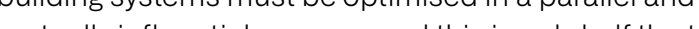
mutually infuential process, and this is only half the task. ecessary measures can only be successfulit they are in line with culturally adapted and socially accepted designs for our bulldings and environments. It is for this reason that solutions for a scientifically identified crisis cannot be solely addressed through science. It requires integrated solutions that are jointly rooted in science and 
16

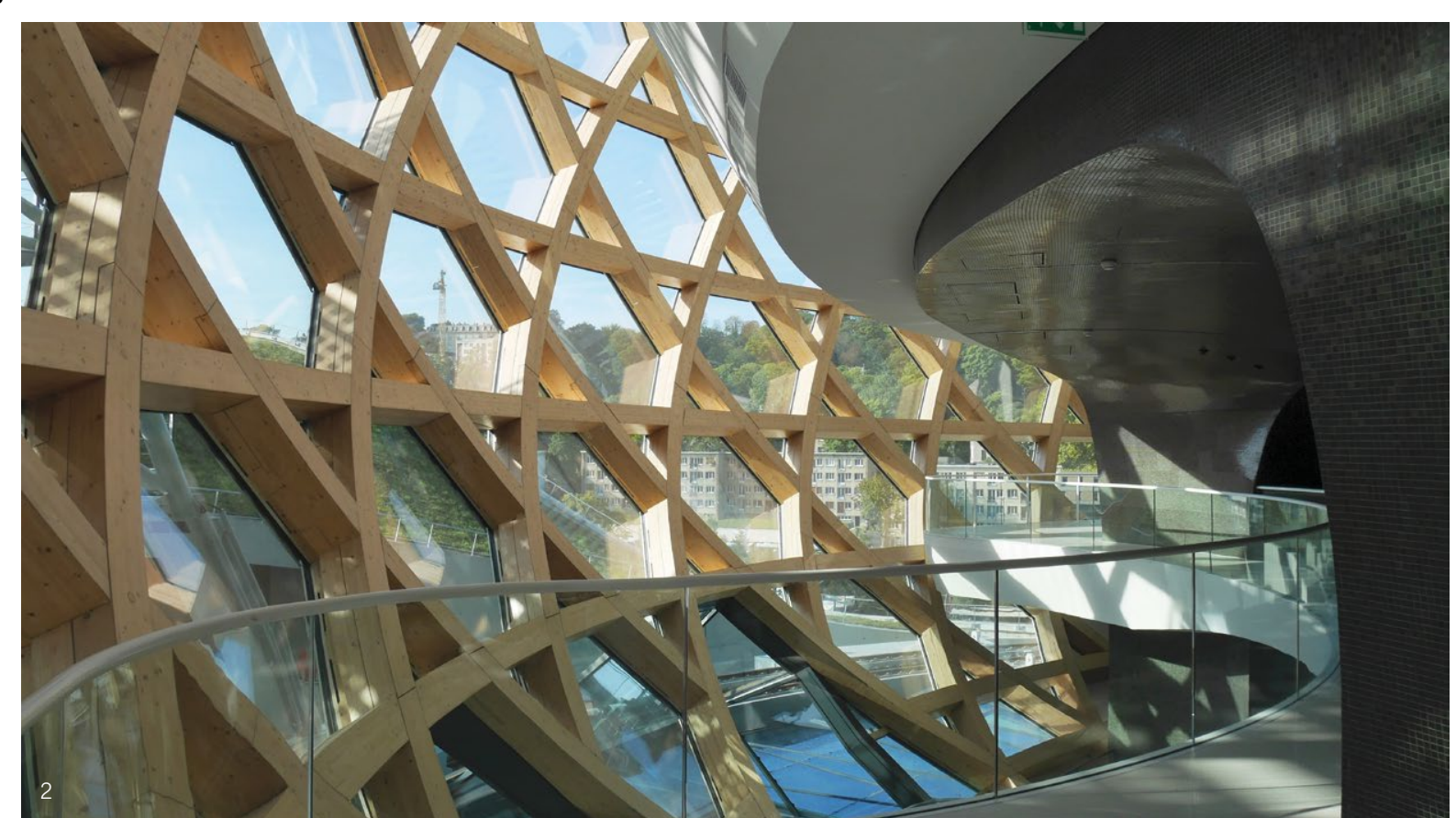

An Unprecedented Challenge

The climate crisis penetrates every facet of the built environment, from its modes of experience to its mechanisms of education; from its lines of enquiry to its pace of development and delivery; and from the needs of initial occupancy to the uncertainty of its use beyond initial occupancy. Underpinning these considerations is a profound shortage of operational skill in the construction sector, where an abundance of advanced tooling and computational capability outweighs the workforce's ability to deliver. In parallel, there is ane demand for a post-disciplinary matrix of cooperative an integrated expertise that operates in a realm of

heightened knowledge exchates and holistic research.

heigh strugoling to cope with these pressures. Aside from struggling to copew these pressures. Aside from proming of today's research is predominantly organised within disciplinary silos. Building sciences have a tendency to limit themselves to measuning incremental changeimprovement - among existing planning methods, manufacturing processes and building systems in tested, often neat, conditions. Success criteria are logic-based and recognised or accepted by means of peer review in established journals and esteemed scientific awards, where discourse on social or historical relevance rarely takes place. On the other hand, design research is often misunderstood as being non-scientific and 'weak', and is dismissed as playing a negligible role in innovation and advancement. Here, it is argued that research developed within a single discipline will
2. La Seine Musicale, Paris. Architects,
Shigeru Ban and Jean de Gastines. Fabrication: Design-to-Production.

3. Bespoke Workstation by Arthur Prior,
The Bartlett School of Architecture, UCL. inevitably be limited and incapable of fully addressing multifaceted problems that are not only technical, but social, cultural and economic.

Furthermore, if climate change is teaching us anything, it is that its cause - and, more so, its consequences - are not yet entirely understood. It is a deeply complex set of conditions, rooted in global human behaviours that are far from simple to 'solve'. This crisis emanates as much from cultural and political values as it does from industrial or agricultural processes. In this regard, design research as transdisciplinary, open to contribution, collaboration, context and wide-ranging scrutiny by multiple communities of practice, is a vital strength. Design Transactions: Rethinking Information Modelling for a New Material Age is thus shared on the premise that radical innovations are needed in all areas of technology and society, not only in the practice of planning and building but also in how we exchange and develop ideas and infrastructure for sustainable and resilient environments.

\section{A Change in Approach}

In response to these matters and more, new approaches to teaching and research are required that place collaborative working and hybrid expertise at the centre of knowledge acquisition and transferability. Innovation wough research and education cannot happen solely within singular disciplines or institutions, but will emerge at the interfaces where different cultures, interests and perspectives are directly confronted with each other. Such new approaches require us to cross boundaries between academic disciplines in physical and social sciences and in humanities, while also bridging the gap between basic and applied research. Only approaches such as these will lead to discourse that question established methods and enables radically new outcomes to emerge.

Architecture and engineering are uniquely placed to face and coordinate this challenge together, with both disciplines underpinning each Innochain project so that boundaries between them are erased. Their shared domain is design research, and its core competence lies in the synthesis and integration of the most diverse and often divergent requirements in the built environment. Design research has the power to transfer scientific, historical and cultural knowledge into artefacts that can be experienced, thus simultaneously initiating expert and public discourse on innovation and its trajectory. Design research is precisely the core competence and potential that is needed by science and industry to resolve future built-environment problems, offering

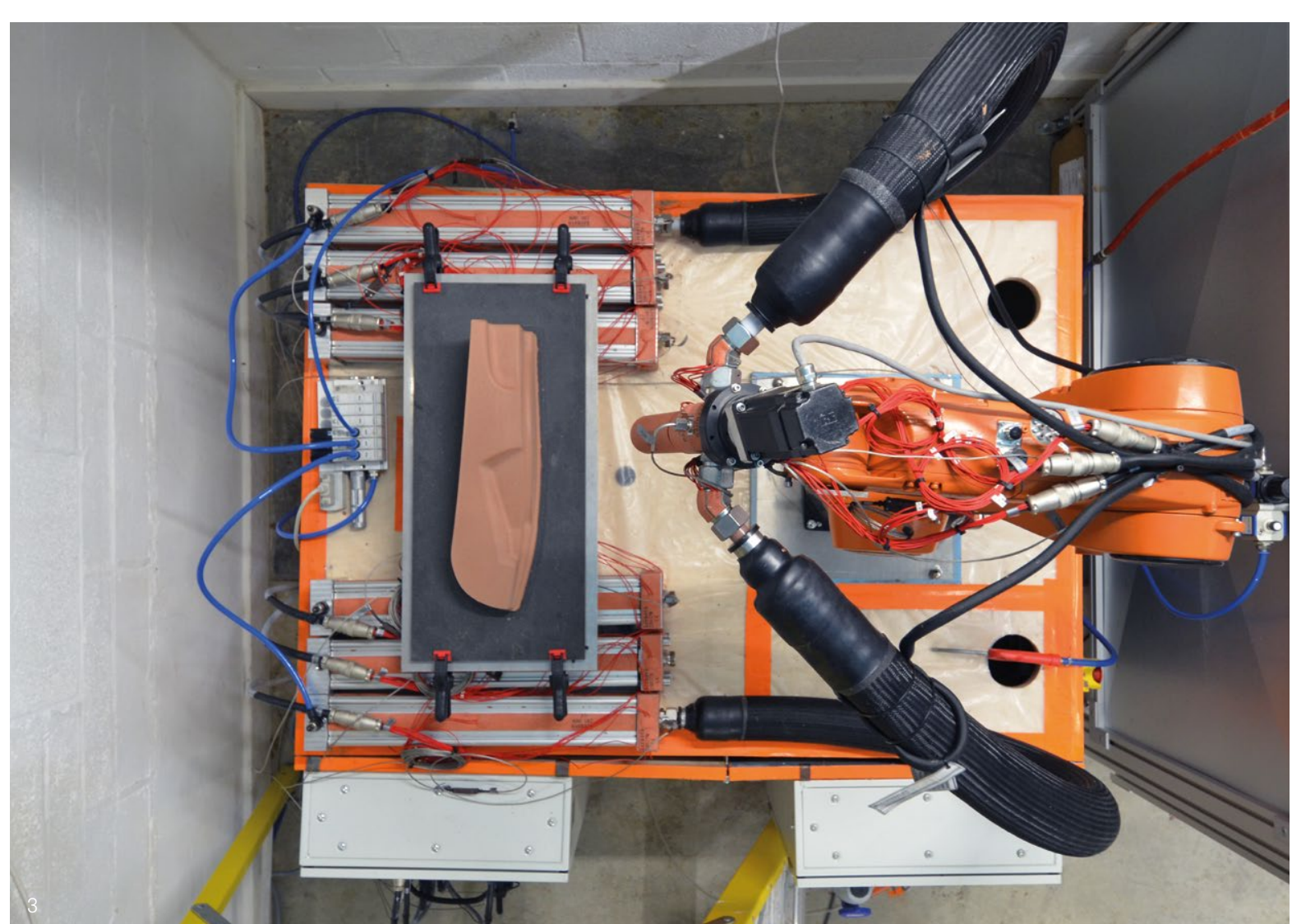




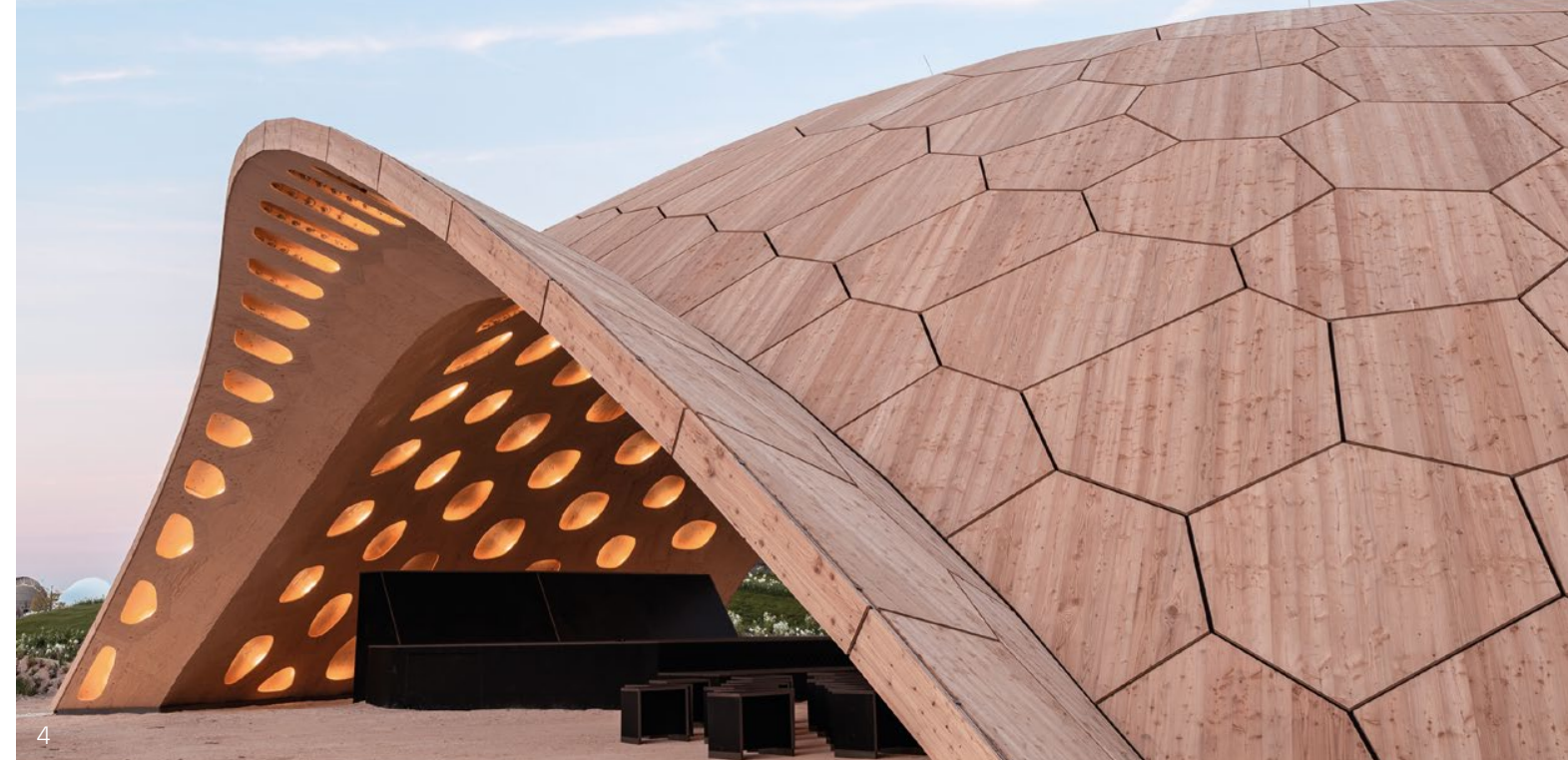

orientation in terms of content and organisation in terms of structure. In this sense, the Innochain network becomes a template for transnational research, industry and practice partnership.

\section{A Collaborative Consortium}

All of the six academic partners from six European countries not only have different professional and cultural backgrounds in the realm of architecture and engineering, but they also represent different modes, scales and outlooks on research, education and engagement with industry. From independent academies of experimental design in culturally-charged cities such as Barcelona's IAAC, to large, technically-orientated public universities in national industrial heartlands, as the University of Stuttgart: and from internationallyrenowned labs within nation a ntres of excellence such as CITA at the Royal Danish Academy of Fine Arts, Copenharen to acclaimed depatments within arge multidisciplinary universites, such as The Bartlett School of Architecture UCL, the In The Bartlett School of Archy unive, enjoys a vast array or parallel networks at its disposal. This framework is a backdrop that merges artistic creativity with eng beering and manufacturing analysis. In both architecture and engineering, established national and international funding is characterised by a strict separation between academic and industrial applied research. As a result, dynamic and everyday exchange is challenging, and it is often left to the market to decide on the viabilty of new propositions. This vulnerability risks the promising results of basic resea not succeeding - even if successfully tested in an support for the transfer to practice. In this regard,
Innochain has offered a new model by involving partners each investing in research time as a necessary risk as basic as investment in capital and human resources. Each of the 14 industrial partners involved in Innochain has a very distinct background. But, together, their expertise covers the entire spectrum of architectura production, from internationally-operating architectural engineering offices, such as ROK and Cloud 9 , specialised construction companies, such as Design-to-Production and Blumer-Lehmann, and specialised services, such as McNeel, structure and S-Form. Innochain's projects are thus based on the parallel and mutually influential development of design methods and minfluential processes. This approcthods and manufactu separation of planning and extrecution, which is the basis separation of planning and execution, which is the basis process's mactice. Tendering processe alocation civing are strictly organised along this dividins and warochain defined the spinl (fis1) to tackle thes obstas in from the

\section{Intersection 1: Communicating Design}

Current methods for communicating domain-specific separation and discretisation of design control. In recent years, state-of-the-art research has questioned these professional boundaries by creating shared methods that integrate design and simulation. These methods either remain data-heavy - and, therefore, unintuitive and at odds with design creativity - or they borrow from firms, such as Foster + Partners, BIG, BuroHappold and Henn, to medium-scale experimental architectural or knowledge in building practice assume disciplinary

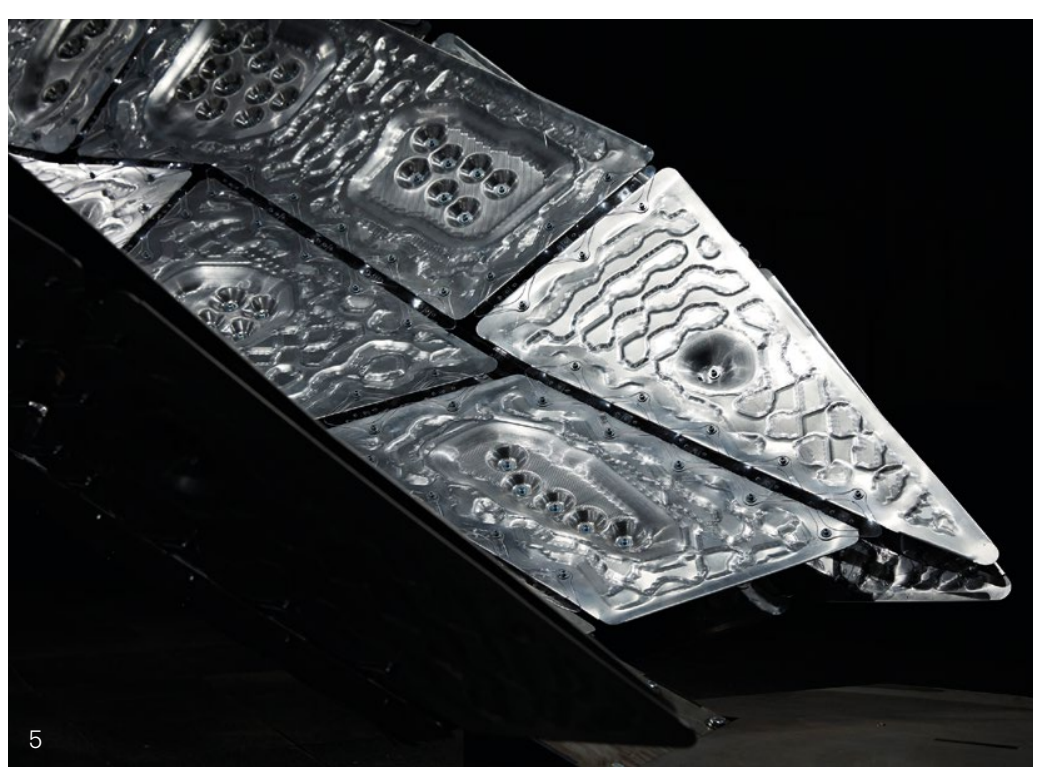

4. ICD/ITKE BUGA Wood Pavilion.
O) ICD/ITKE, University of Stuttgart. 5. A Bridge Too Far by CITA at the Royal Danish Academy of offer potential for innovative links between structural simulation, material development and design modelling. Integrating isogeometric analysis, for example, allows us to address issues of disciplinary collaboration by enabling interfacing of verified high-end Finite Element Analysis (FEA) with standard CAD design tools. Integrating material simulation allows us to address issues of design-phase integration by interfacing early-stage design development with material analysis and the activation of material performance, while integrating building physics for performance control enates to extend the simulation and activation of material performance into the control of adaptable structures in real time. Innochain was thus charged with developing alternative means to communicate, measure and examine how complex design solutions with multiple criteria could be managed and communicated with non-expert stakeholders across the design chain. Tasks carried out at this intersection included:

- Integrating isogeometric analysis. Academic Lead: Institute for Building Structures and Structural Institute for Building Structures and Structural
Design (ITKE) at the University of Stuttgart. Industry Design (ITKE) at the Univer

- Integrating material performance. Academic Lead: Integrating material performance. Academic Lead: Centre for Information Technology in Architecture Partners: Blumer-Lehmann, White Arkitekter.

- Integrating building physics for performance control Academic Lead: Institute for Advanced Architecture Academic Lead: Institute for Advanced Architecture of Catalonia (IAAC). In
Foster + Partners.

- Multi-criteria optimisation in early design phase. Academic Lead: University of Applied Arts Vienn Industry Partners: str.ucture, BIG.
- Alternative means to communicate measure. Academic Lead: The Bartlett School of Architecture, UCL. Industry Partner: HENN.

Intersection 2: Simulation for Design

Simulation for design has significant implications for the pace and effectiveness of iterative information as well as fabrication outcomes. Virtual Common methods for simulation in the built environment assume single-scale engagement across separate phases and exclude the simulation of material and fabrication processes. Recent research identifies new opportunities for simulation to link the design of material with the design of structures. This creates new implications for material deployment That necessitate new methods for analysing specifying and controlling fabrication.

Following this path a number of Innochain projects examine how simulation can be used as a means to cross between scales and synthesise material performances with machine-driven procises. Multi-scalar modellin and the simultion of anisotropic materal pe modelling for ine simulation of an for instance, offer a design framework for the innovative use of known materlals. Sim purpore of trilling ing purpose of trialng innovative materialconsiguration, formusk offers the potentia to examine the such as fibre-reinforced polymers. Simula ing

. hydraulic forces of the liquid material and the formwork that counterbalances them, while innovative simulation of robotic feedback reveals novel digital fabrication strategies that may incorporate real-time feedback and mechanical or compositional changes in the material. 


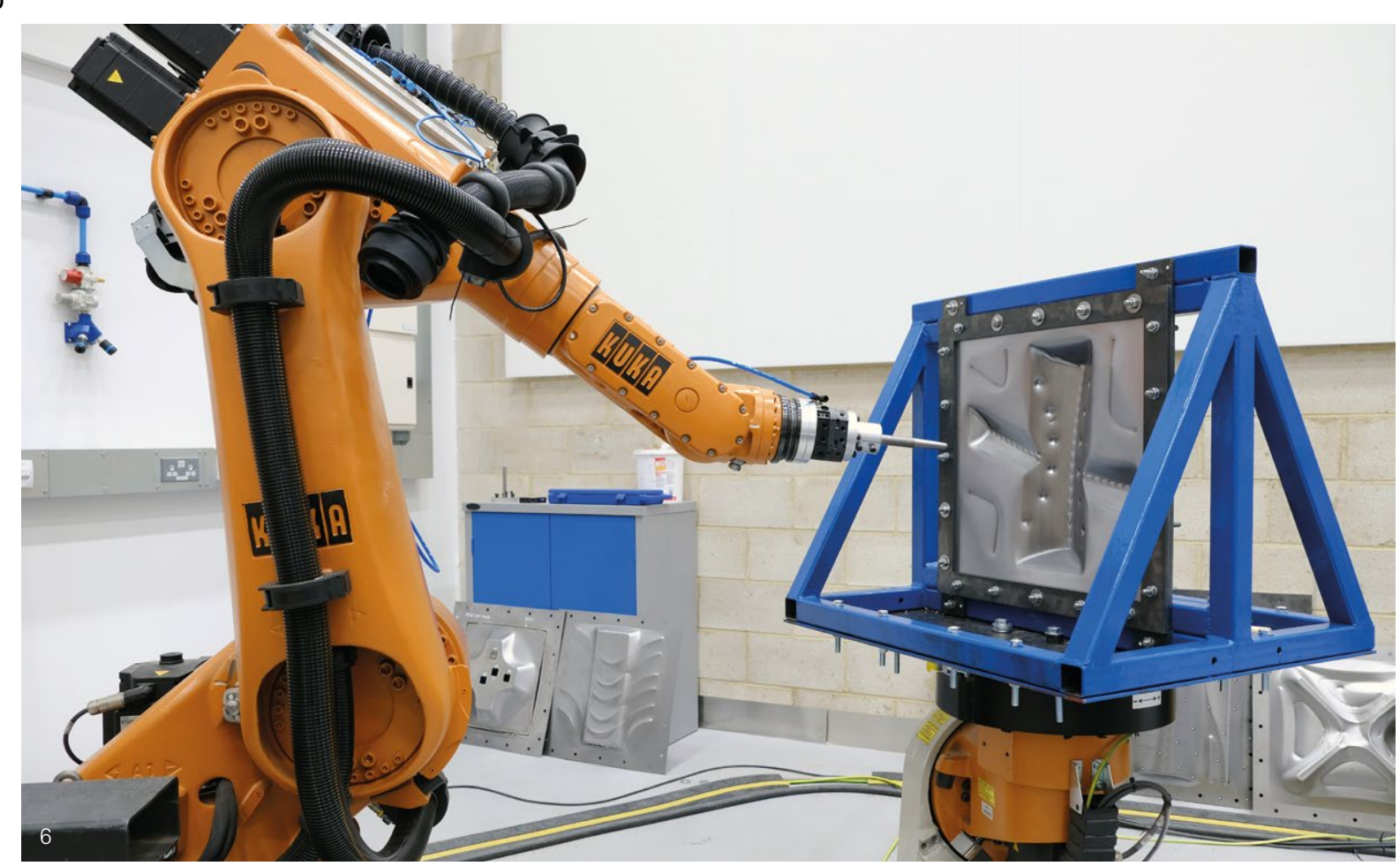

- Multi-scalar modelling for building design. Academic Lead: Centre for Information Technology in Architecture (CITA), Danish Royal Academy of Fine Arts. Industry Partners: BuroHappold.

- Simulating anisotropic material. Academic Lead: University of Applied Arts Vienna. Industry Partners: Cloud 9, Blumer-Lehmann.

- Virtual prototyping, fibre-reinforced polymers. Academic Lead: ITKE at the University of Stuttgart. Industry partners: S-Form.

- Simulating concrete formwork. Academic Lead: KTH Royal Institute of Technology. Industry Partners: BuroHappold, White Arkitekter.

- Simulating robotic feedback. Academic Lead:

The Bartlett School of Architecture, UCL. Industry partners: ROK, Foster + Partners.

\section{Intersection 3: Materialising Design}

Current methods for materialisation within the building industry are overwhelmingly based on mass production They rely on the standardisation of material and

fabrication to afford control and optimise material use. With ilgtisation, these methods have become outdate and new models for material optimisation have emerged. Where subtractive digtal fabrication techniques have matured and been applied to realise complex buildings, recent research efforts utilise bespoke machines or industrial robots as general fabrication tools for additive abrication to innovate mass-customised materials with designed performances.

Innochain's focus in this domain focuses on trialling fabrication and planning methods for new designed materials that embed material optimisation within their composition. Concrete printing investigates how printing technology eliminates the need for formwork to create a more variable and precise concrete tectonic. Material gradient fibre-reinforced polymer (FRP) and applied robot-controlled material deposition investigates how fabrication prod material structural and environmental properties. Design for manufacture and assembly (DfMA) develops innovative planning processes that interface new material practises with the requirements of industria material practises with the requictial from the factory to onsite production. Tasks carried out

- Concrete printing. Academic Lead: KTH Royal Institute of Technology. Industry Partners: Foster + Partners.

- Material gradient FRP. Academic Lead: ITKE at the University of Stuttgart. Industry Partners: str.ucture and S-Form.

- Applied robot-controlled material deposition.

Academic Lead: The Bartlett School of Architecture, CCL. Industry Partners: Foster + Partners,

- Design for manufacture and assembly. grade material deployment for the purpose of varying Arts. Industry Partners: Design-to-Production, Blumer-Lehmann.

Small-scale robotic manufacturing for large-scale buildings. Academic Lead: IAAC. Industry Partners: Cloud 9, ROK.

Innochain: A Template for

Research and Practice Collaboration

All Innochain participants, including academics,

industry partners, research fellows and their respective

project associates, have been engaged in the project's

fundamental aim of contributing to interdisciplinary

and multi-sector operations across the digital chain.

Focusing on innovation in practice has meant th

research has not been limited to technological

innovation but has also explored the potential of

novel implementation of known tools and processes

to create new products and solutions offering capability

to address the sector's primary challenges. In this regard,

the means through which we mutually transact our

information and share our expertise - be it the

adaptability of modelling information, the analysis

of material performance or the integration of as-built

with as-designed data - are fundamental to building an

environment for the future that is sustainable, resilient,

culturally enriching and diverse in its experience.

\section{Bibliography}

Knippers, J., 2013, 'From Model Thinking to Process Design' in Architectural Design, ol. 83, No. 2, p.74-81.

Knippers, J., 2017, 'The Limits of Simulation: Towards a New Culture of Architectural Engineering in Technology, Architecture

Knippers, J. and Speck, T., 2012, ‘Design and Construction Principles in Nature and Architecture' in Bioinspiration \&

Menges, A. and Knippers, J., 2015, 'Fibrous Tectonics' in Archisec
No. 5, p.40-47.

Sheil, R., 2005, 'Transgression from Drawing to Making in Archinectural Research

Q 2012 Manufacturing the Bespoke: Making and Prototyping A hitecture, London, Wiley.

Sheil, R., Glynn, R., Menges, A. and Skavara, M. (eds.). 2017, Fabricate 2017: Rethinking Dress. 
Design Methodologies:

Rethinking Transactions

\section{Between the Drawn and the Made}

Bob Sheil

The Bartlett School of Architecture, UCL

The Bartlett Schoo
Mathilde Marengo

Institute for Advanced Architecture of Catalonia (IAAC), Universitat Politècnica de Catalunya

Ulrika Karlsson

KTH Royal Institute of Technology, Stockholm

\section{Introduction}

\section{Bob Sheil}

The Bartlett School of Architecture, UCL

Neither architects nor engineers tend to make buildings, instead they make information that is used by others in the production of buildings and the built environment.

As an instruction to make, design data differs in its intent, accuracy, and role in the process of making, and system tasked with the challenge of making will remake design data as a rehearsal for manufacture and as a means to verify what was made and what instructions it followed. Rather than act in pertnership and union, the construction industry is fraught with obstacles and barriers that prevent the evolutionary flow of data instead imposing a stop and start and highly inefficient rhythm. Design Transactions: Rethinking Information Modelling for a New Material Age gathers projects that seck to intervene, challenge and innovate in jects that They share an experimental methodology focussing on design-h-by-design methodology focussing on design-led physical exphasis allows the researcher to engage dir the investions the rectly with the investigated techniques and technologies moving along the digtalchain from design and analysis to specilication and fabication. Such an integrated approach positions each research enquiry within a network of interconnected expertise and practice that collaborates between academic, industry, and practice, providing the opportunity for research teams across the network to share tested, analysed and evaluated results. Design as a method of enquiry is a reflective practice in which the researcher engages in a dual mode of
Scientifically, each physical experiment acts as a material research enquiry (Duits, 2003) by which the concepts and technologies of the research enquiries are evaluated. Moving between the exterior and the interior of making, design creates a conversation between the dissective action of analysis and critical assessment and the creative action of proposition and result. The method is relevant for design-led research in architecture and engineering as it ties design creativity to research

investigation. Building practice has been described as a wicked problem, in which the "information needed to understand the problem depends on one's idea for solving it' (Beim and Ramsgaard Thomsen, 2011). Design is therefore an active process by which the designer develops the dimensionality of solution in context of a given problem. Solutions are assessed not absolutely as true or false, but rather assalitatively as better or worse. To employ a research-by-design methodology therefore allows the individual research projects to engage with the solution-lid processes of creative trouble shooting that chan creative trouble shooting that characterise the design process. From here, the discussion is taken up by Architecture of Catam (IAAC), Unive Ad Pollecnica Architecture of Catalon the InAC), Universitat Politècnica of Technology (KTH), Stockholm.

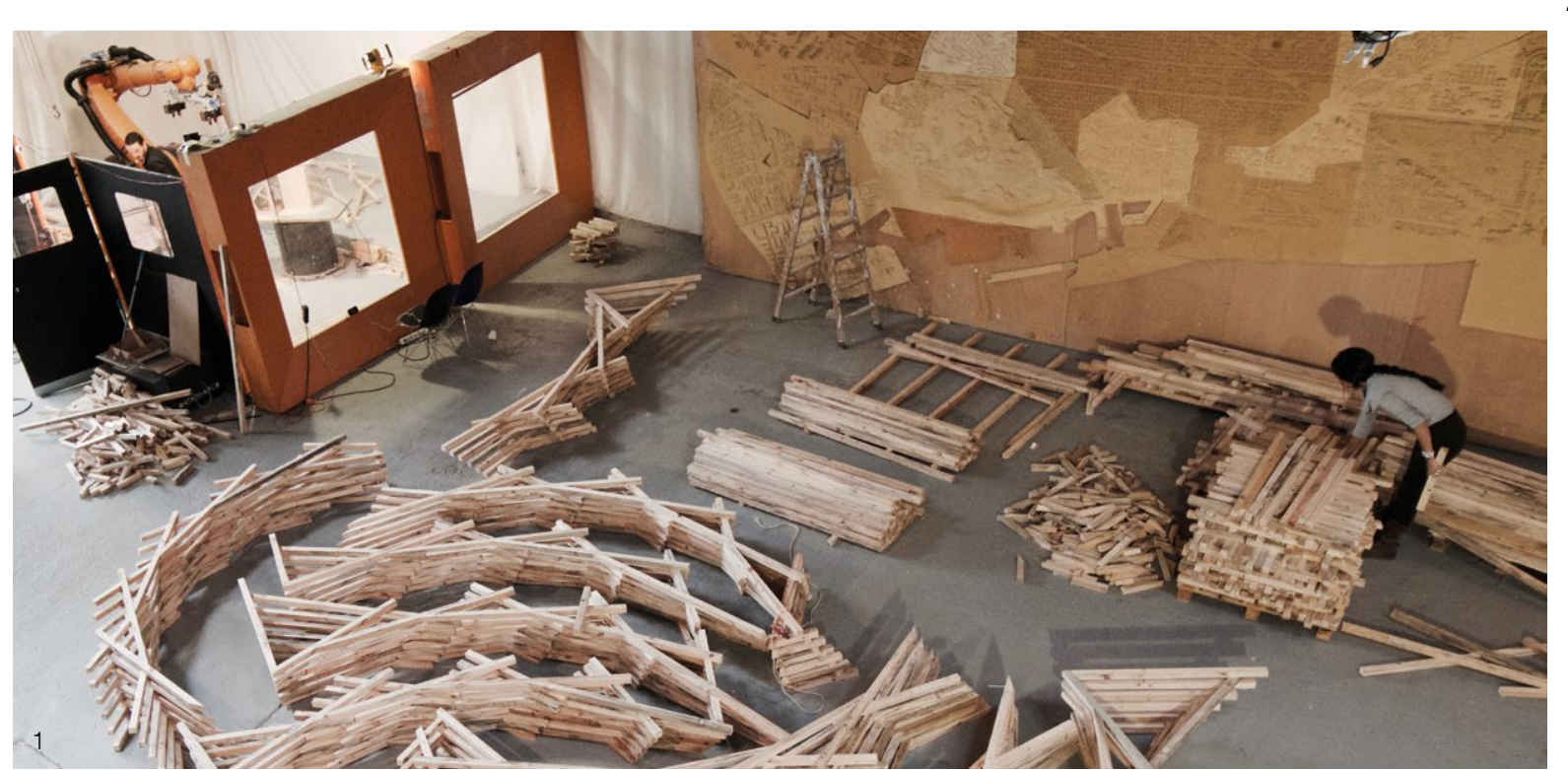

Situated and Experimental Research Protocols Integrating Academia and Industry within the Construction Sector

Mathilde Marengo

Institute for Advanced Architecture of Catalonia (IAAC), Universitat Politècnica de Catalunya

Today, development and innovation in technology are taking place at an unprecedented pace and are drastically changing the way we live, by introducing augmented design solutions and applications. Across social habitats and habits, political decisions and many market sectors the impocts, pof technolosical development is und sectors, Yet, within this context, the construction sector lis bet, within this contiont, teconstruction sector lags this, stating that it is among the least digitised sectors worldwide (Barbosa 1 2017) It is estimated that the integration of disital technologies including advanced automation, data optimisation, onsite execution and the upskiling of the workfoce has the potentialto boost upskillng of he workforce has the potenthe to "boost productivity in construc

response, new research foreseeing technological trends and their transformative potential are being produced and developed in academia, practice and related startups. Fundamental to their success is their integration with the construction industry, as no matter how hard scientists work, our impact will almost always be limited to our immediate academic clicles if our results never catch the altention of those who have the power to act on them' (Safford and Brown, 2019). Although many such enterprises are already under way. connecting new tools through multidisciplinary 

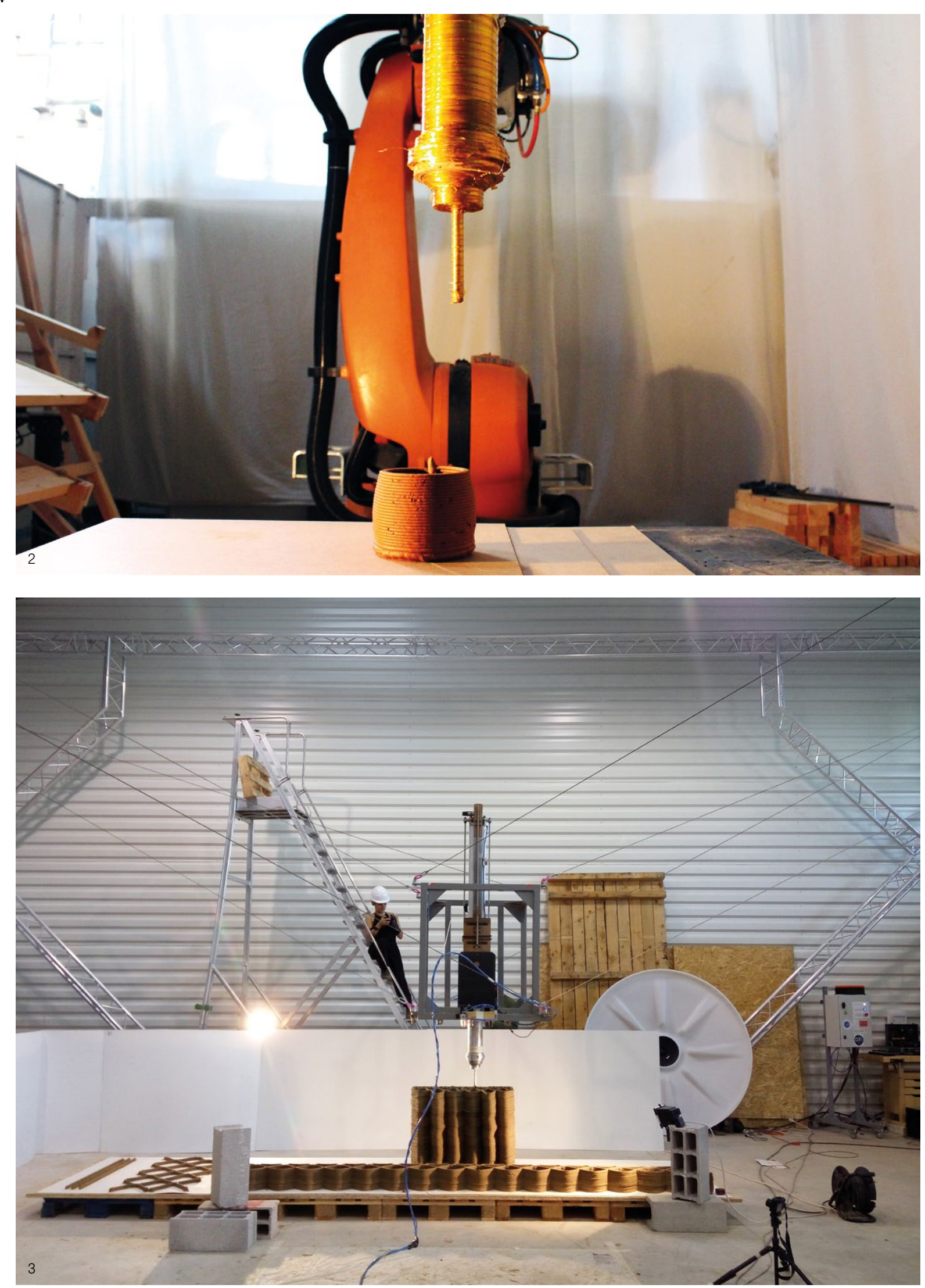

frameworks and approaches, their practical impact is yet to be seen on a wider scale in the construction industry. Nonetheless, the potential of these collaborations, in relation to the introduction of emerging digital tools within novel actuation protocols for construction companies, is evident in the significant productivity gains and sustainable results they present (Blanco et al., 2017). Innochain strives to bridge this gap by merging design, research and production through the consolidation of a training network for Early Stage Researchers (ESRs) developing situated projects through an experimental methodology at the intersection of academia and the construction industry. More specifically, the focus lies within the challenges and opportunities that occur as the new digital chain is established, allowing enhanced tools and concepts to be developed thanks to an interdisciplinary approach, which integrates knowledge and practice from neighbouring fields. This is elaborated through the identification of the three major challenges identified - communicating, simulating and materialising design - and has thrived due to the integration of experimental and

interdisciplinary collaborative approaches.

On this basis, relationships between academic and industry partners within the Innochain network are consolidated, and possess transversality in relation to the construction industry. From material development and manufacturing enterprises to leading design and engineering firms, the large number of industry partners as design-active agents in building practice allows the network to reflect the multidisciplinary and multi-scal fundamentals of research through the digital and physical realms of practice.

Through the development of situated and experimental research and training activities and demonstrators, the network questions the linear process with which the construction industry - like many productive industries - is associated, and creates an opportunity

for the application of circular principles to this field.
This allows the projects to demonstrate the importance f a systemic, holistic and integrated approach based on working with design through research and research by design. In addition, experimentation and testing in context operates as a pretext for innovation within the igital chain.

The work offers further resonance for education, reviewing and renewing models in academia in line with principles emerging from the research. This can be appreciated through actions such as the Computation Bamboo installation developed and built in July 2018 at the IAAC Global Summer School in Quito, Ecuador, with Innochain researcher Evy L. M. Slabbinck based at the Institute of Building Structures and Structural Design (ITKE), and projects at the City Intelligence Lab of the Austrian Institute of Technology, opened in October 2019, led by Innochain researcher Angelos Chronis. Through Probes, Pavilions and Exhibitions, the network has taken the scope of the project beyond academic and industrial realms. As demonstrated by the Elytra Filament Pavilion by ITKE and ICCD Stuttgart, commissioned by the V\&A museum in 2016, which engaged Innochain researcher James Solly whose work on Coreless Filament Winding (CFW) and their integrated simulation and fabrication strategies is discussed later in this book. Likewise The Bridge Project by Innochain researcher Tom Svilans of CITA with industry partners White and Blumer-Lehmann, which developed an experimental design protocol, informed by the simulation of material performance and integrated feedback loops.

Beyond providing a unique opportunity to merge innovative research practice with industry impact the Innochain network demonstrates the importance of situated, shared and experimental research protocols within the construction sector. Innochain has defined a circular system combining digital tools, mas defin resources, design protocols, advanced manufacturing and onsite operations.

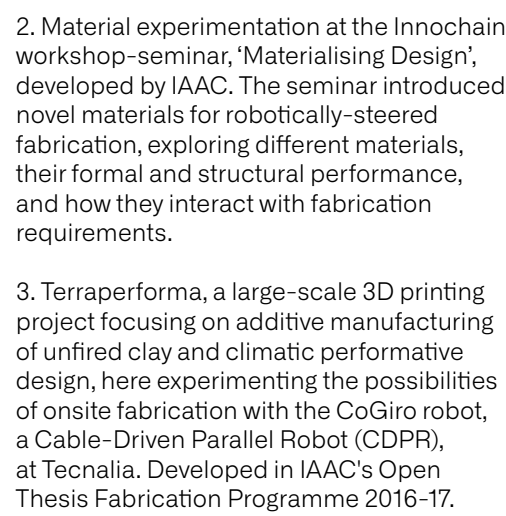




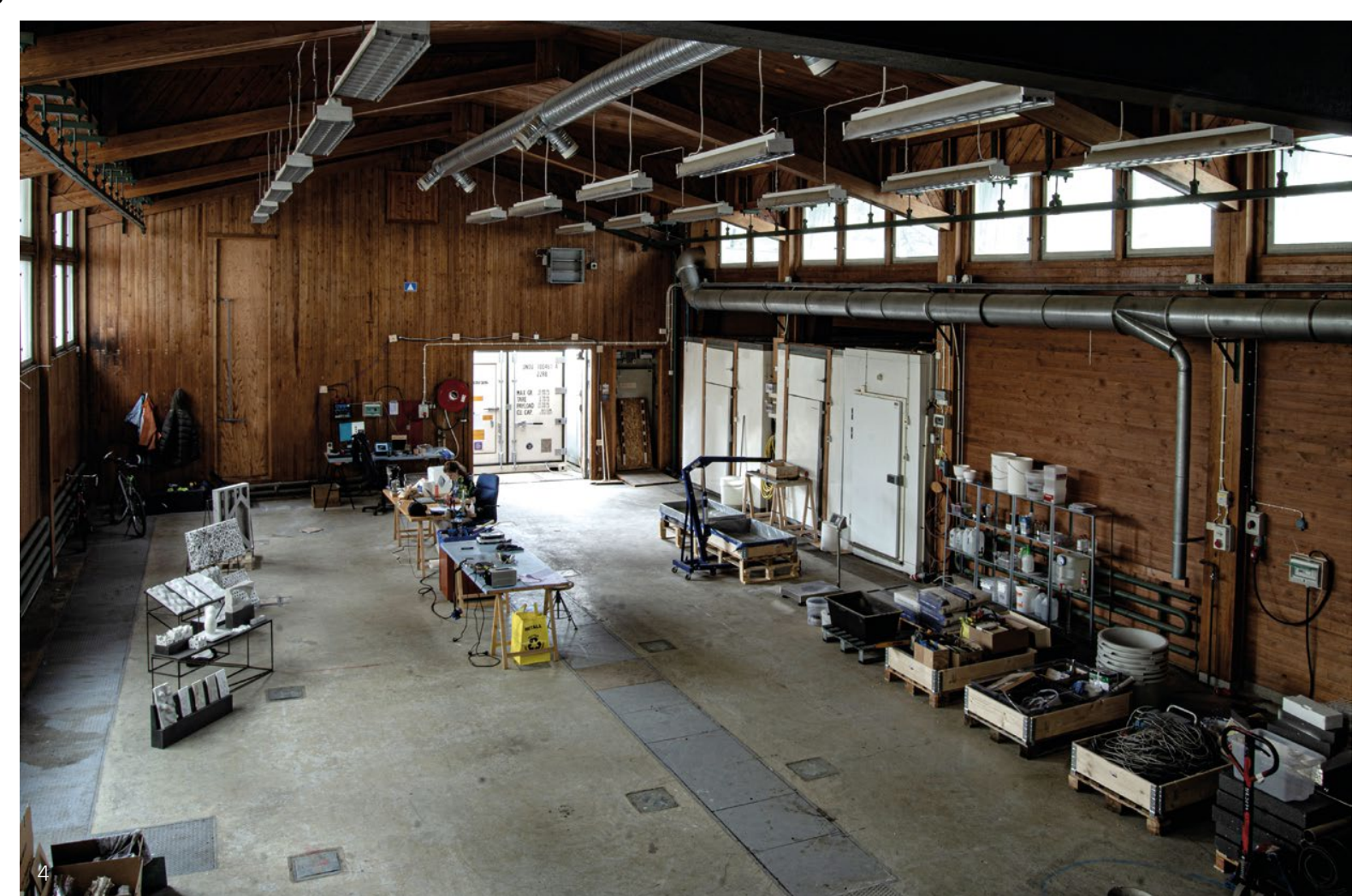

Displacement of Effort

\section{Ulrika Karlsson}

KTH Royal Institute of Technology, Stockholm

The architect and historian Robin Evans described the position and space in which the architect operates on Unlike a painter or sculptor the architect is always working on some intervening medium, often a drawing selking on some intervening medium, often a drawing, or the material of the outcome. At first. Evans found this displaced position to be to the architect's disadvantas displaced position to be to the architect's disadvantage. But he soon observes the the ach played by the intervening medium iself, in this case 'd displaced" effort of an archilect might not always be a disadvantageous position, and hat the two differen approaches to 'work' might not be incompatible at all. Yetnot al things architectural can be derived from drawing. Several of the projects win the Innochain research programme have methods that engage both these positions. This text takes a closer look at one of them (also described on $0.122-127$ and p.134-135 of this volume), where ice takes the role of formwork. A refrigerated container is attached to a full-scale material lab at the KTH Royal Institute of Technology
Stockholm campus, providing the infrastructure for an
4. Ice Formwork project by Vasily Sitnikov. Photo: Vasily Sitnikov.

5. Drone Spraying by Stephanie Chaltiel. Photo captured by drone camera at Practice
Futures - Building Design for a new Material
Age exhibition, KADK, 2018.

6. Concrete Deposition by Helena - Building Design for a new Material Age exhibition, KADK, 2018. Photo: Anders
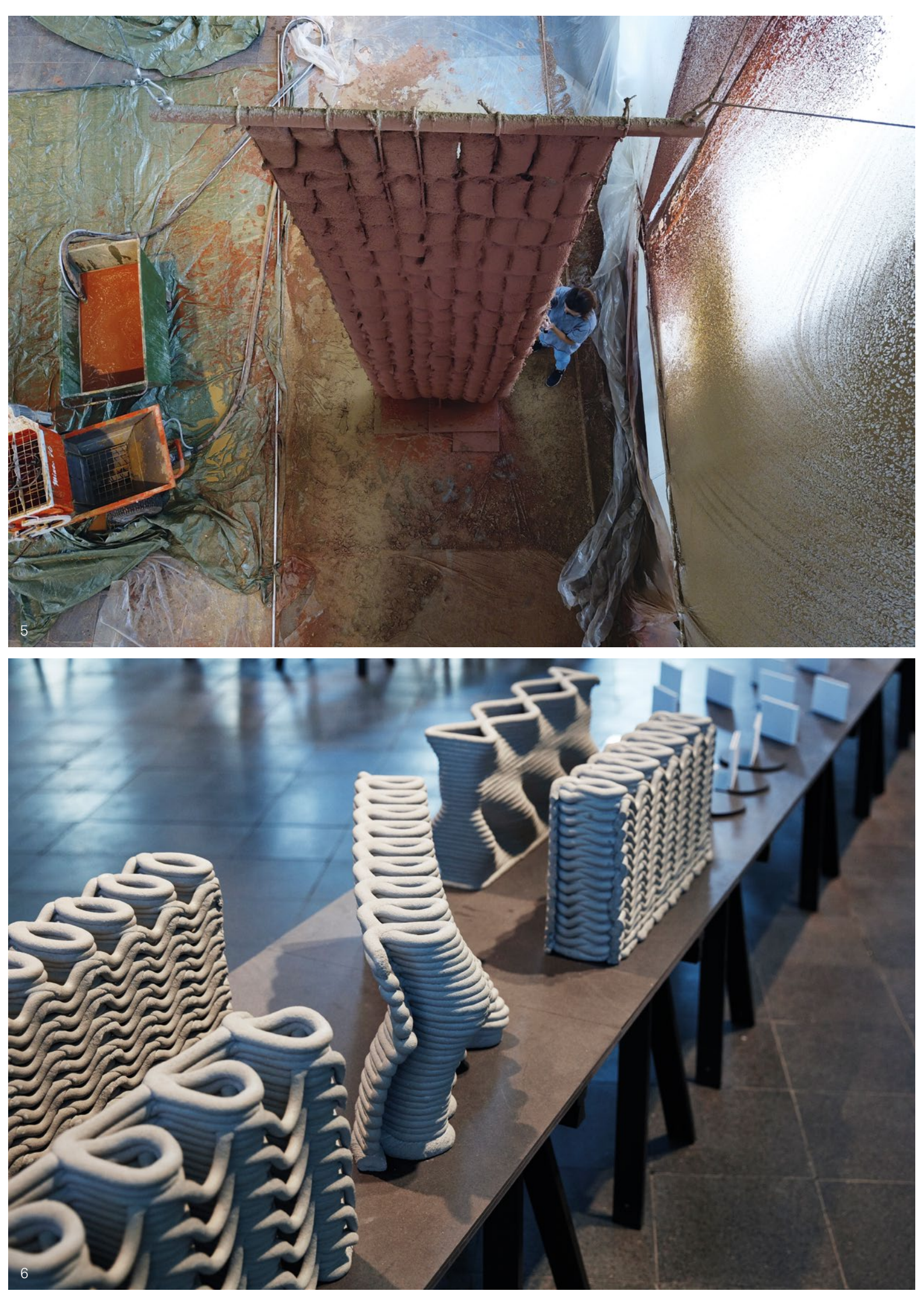
alternative material system for the fabrication of pre-cast concete elements using CNC-milled ice as formwork. The formwork consists of a mould and a counter-mould made entirely out of CNC-manipulated ice. This research project, led by the architect Vasuly Sitnikov, challenges conventional modes of fabrication in which material waste and manual labour lack resourceful considerations. The carefully designed and arranged setup simulates or stages a resourceful manufacturing process of concre elements, where the traditional formwork material of polystyrene foam has been replaced by a phase-

changing material: ice.

First, this method of developing an infrastructure for a full-scale fabrication system removes the need for intervening medium. It requires weighty physical work and management that give the architect an opportunity to have direct contact with the object of thought and the corporeal material of the outcome. In this case, this means direct contact with, or proximity to, the transformation between states of fluidity and solidity, which are germane to casting. A fabrication method for geometrically-articulated concrete elements. At the same time, there is an intervening medium: a setup and infrastructure that translate a stack of notations into different carving depths in ice, preparing a series of moulds of ice for concrete casting in climatically-controlled and interlinked rooms. A standard $6 \mathrm{~m}$ refrigerated shipping container contains a custom-made three-axis computer numerical control (CNC) milling machine, a cyclone extractor to remove the ice dust (the by-product of its milling), equipment for production of low-defect ice stocks, storage of concrete raw materials and a concrete pan-mixer. The container is linked to a material lab at KTH that, for this project acts as a control room. This setup is designed fornon-human technical and material processes and is linked to a staffed operating room. The assembly of this lab has made it possible to work in direct contactwis the material of the outcome - the ice formwork and the cast concrete elements - as well as with the intervening mediual fabrication system.

for fabrication method is not full-scale and is merely prototypical; however, it uses an industrial system for fabrication. The architectural researcher is still working from a position that is slightly withdrawn from the anticipated industrial object, and, as a result, there is a displacement of effort. This provides a generative space from which iteratively test and modulate the setup of the lab. The carefully crafted and constructed infrastructure or model acts as a basis for running physical simulations. Thus there is potentia for the two observational approaches to 'work', a) the researcher working in drectcontact thith the object of thought and the material of the outcome and b) through an intervening medium

The displaced effort of the architect when working on an intervening medium, in comparison to working in direct contact with the object of thought, constitute different positions for practice-based research or research in the making. In the ice formwork research project, the toolpaths that the CNC follows are projected through space so that the tip of a cutting tool produces an anticipated form out of the ice. This process produces a mould and a counter mould, in which negative form is translated into concrete. What this suggests, is that instead of a conventional linear process of refinement, we can develop multiple levels of feedback from early stages of design and manufacturing. It is worth reflecting on the particular condition in which architectural knowledge is produced - in this case, through the staging of an infrastructure for the automated process of fabrication of architectura elements. The position and space in which the architect operates are through an intervening medium and through direct contact with the material of the expected outcome. Both this method and the Innochain project as a whole blur the spetial bounchies between the disciplines of architecture, craft, eng

\section{Bibliography}

Barbosa, F., Woetzel, J., Mischke, J. Ribeirinho, M.J., Sridhar, M., Parsons, M., Bertram, N. and Brown, S., 2017, Higher Productivity, Washington DC, McKinsey Global Institute.

Beim, A. and Ramsgaard Thomsen, M., in Architectural Research: Drawings, Models, Experiments (eds.)., Copenhagen,

Blanco, J.L., Mullin, A., Pandya, K. and Sridhar, M., 2017, The New Age of Engineering and Construction Technology.
www.mckinsey.com/industries/capitalprojects-and-infrastructure/our-insights/ the-new-age-of-engineering-andconstruction-technology (accessed

Duits, T., 2003, The Origin of Things: Sketches, Models and Prototypes:

Evans, R., 1986, 'Translations from Drawing o Building' in AA Files, no. 12 (Summer), of Architecture, p.4-5. Rittel, H. and Melvin W., 1973, 'Dilemmas
in a General Theory of Planning' in Policy
Sciences, Vol 4 , p155-169, Safford, H. and Brown, A., 2019 , Six Strategies for Sience to Policymakers: Six Strategies for Success', in Nature, Vol.
572, p.681-682.

Schön, D., 1983, The Reflective Practitioner: Temple Smith. 
Perspectives:

Transactions and Trajectories

Bob Sheil

The Bartlett School of Architecture, UCL

Mette Ramsgaard Thomsen

Centre for Information Technology and Architecture (CITA), the Royal Danish Academy of Fine Arts,

Schools of Architecture, Design and Conservation

A New Material Age

We may tend to think of transactions as a binary exchange a deal, a transfer of ownership, the fulfilment of an agreement or indeed an action that assures commitmen While such essential transactions occur in all fields of design, the nature of design transaction as channelled through building procurement has operated in the face of increasingly complex and often contradictory challenges. Regardless of any theoretical orthodoxy, built architectures of numerous creeds have emerged through - and often despite - the circumstances of boom-and-bust. Here we reflect on the notion of boom-and-bust. Herewe refton the notion of transaction through design as this ameol, both outlook vis-à-vis what might lie ahead.

Within the context of design for the built environment what matters most?

Size: the construction industry is vast.

Profile: the industry spans from the individual operator to the global conglomerate.

Context: it is thoroughly dispersed.

Protocols: it is interconnected with every facet of

regulated economies.

Culture: it is entwined in traditions and methods that arc back centuries.

Processes: some are static, others are evolving at unprecedented pace and more are in development in response to demand.

Operational Skills: shortages are deeply challenging. Materials: there are consequences to every selection, from scarcity to resilience, from performance to meaning. Information: there are only pockets of common ground.
Politics: therein lies the power to commission and regulate.

Places: each location for every activity involved has unique coordinates, conditions and aspects. Environment: we know that we are living with finite resources in a changing climate in which the status quo of building culture cannot be sustained.

Definitions of where design and construction practices begin or end are incrementally evolving. The designer is increasingly in command of data, from how components are manufactured to how they are assembled and perform. Likewise, the contractor's expertise is increasingly relied upon at early stages including the formation of concept and ideas. Notions of innovation, creativity and genesis are therefore illusive in their origins, as are any hard boundaries between in their origins, as are any hard boundaries between For such close siblings, all design actions are transactions and, as such, they are open to translation by the recipient, and very often by the author too. Furthermo the ro Furthermore, design transactions are more than mere exchanges. they signify a drection of travel, a trajectory. Connecting research in practice and industry to research in academa, this bock examines how advances in digtal design tools challenge building culure, enabling more sustable, more inforned and more materally-smart design solutions. The work presented here represents a new generation of interdisciplinary research with a strong industry focus that effects real changes in the way we think, design and build our physical environment. Challenging conventional and mainstream approaches toward design as a linear process of incremental refinement, the work identifies
design research potential as a distributed and

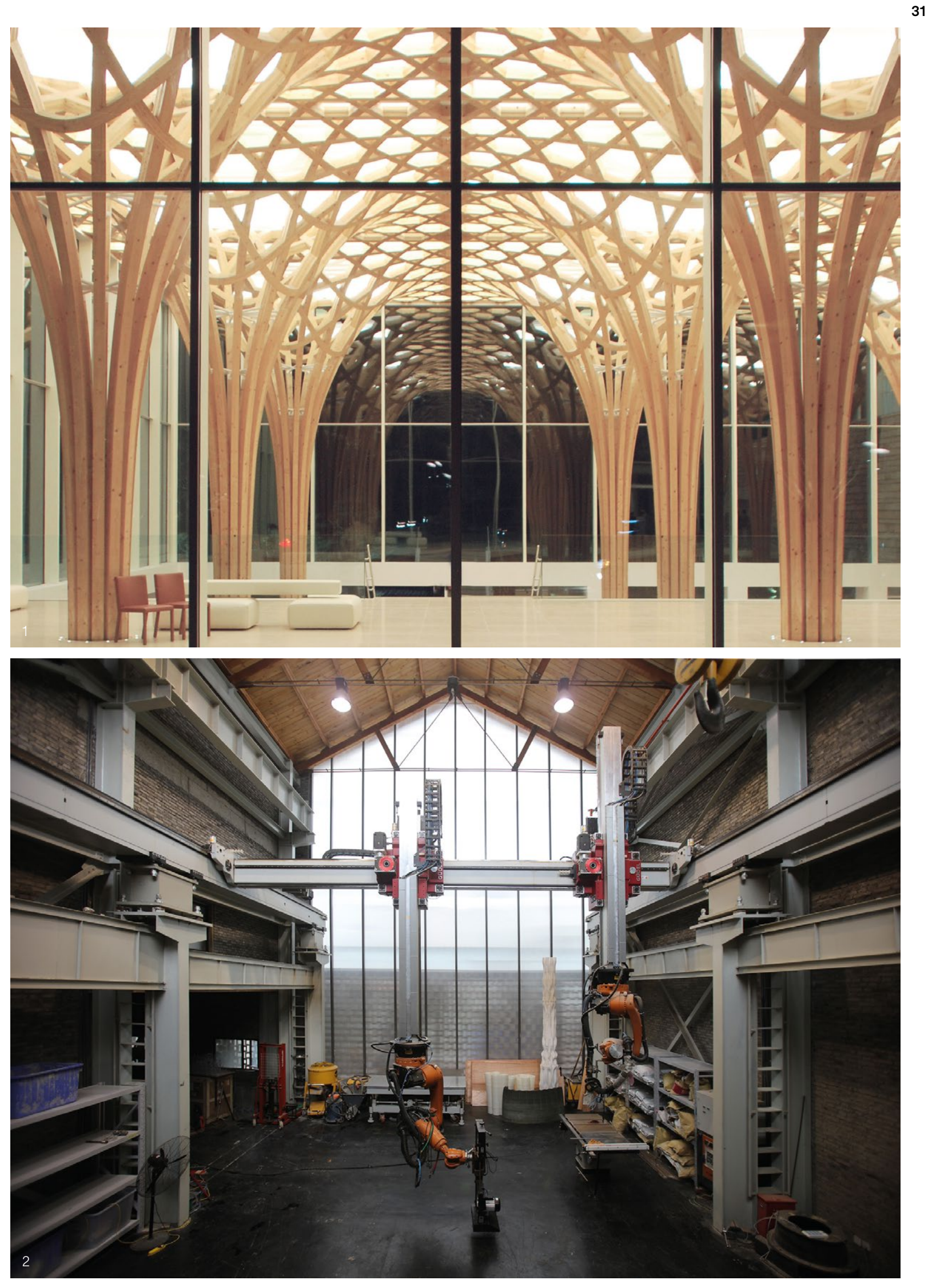


32

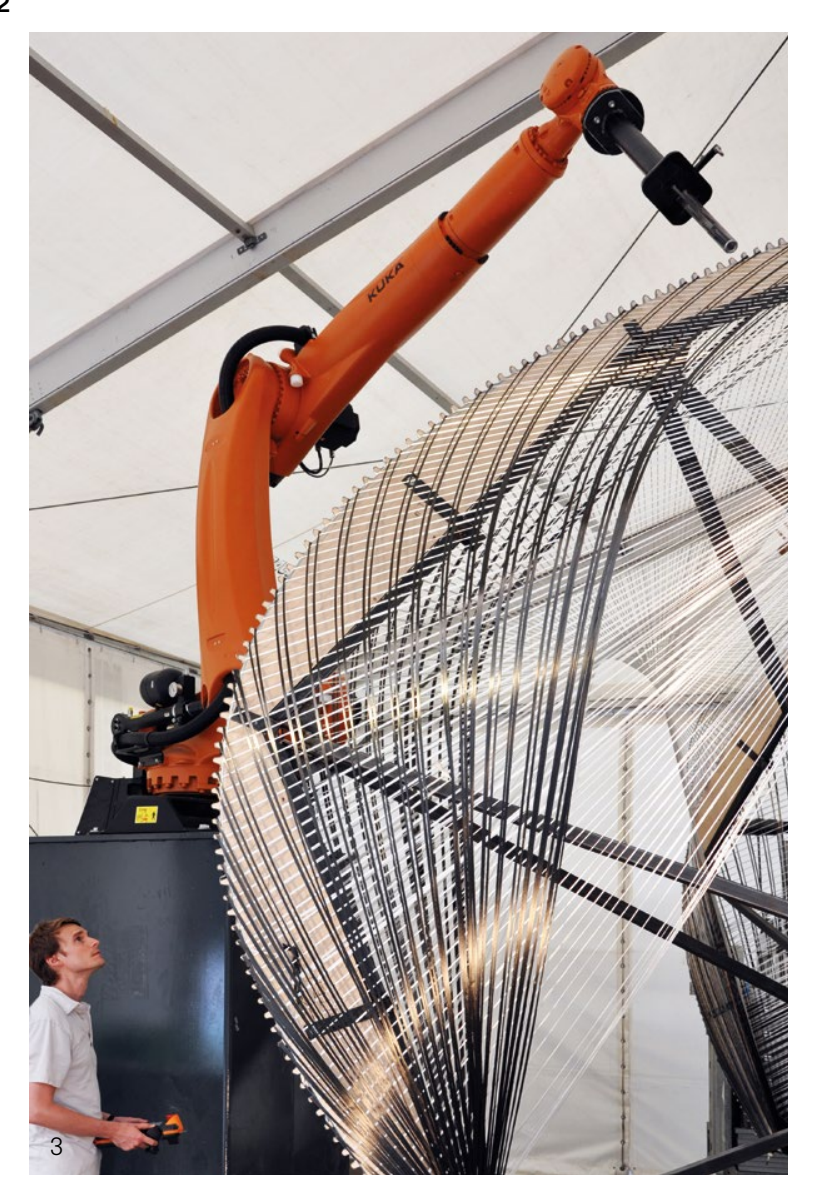

interdisciplinary activity across the entire production chain. From establishing a multi-stakeholder brief to executing seometries on structures that acknowledse and work with their inherent material properties. Situating feedback between design processes as a concern for developing holistic and integrated design methods, the work presented here represents new interdisciplinary design methods that intents new simulation and interface with material fabrication.

New Forms of Practice

Architecture and engineering have equally distinguished legacies that arc back thousands of years. Both took historic step when defining their fields as distinct subjects taught and researched in parallel to professional disciplines, around 175-200 years ago.' Ever since, the relationship between academia and the professions has remained intrinsically linked with emergent poles unique to each field. While engineering in academia has remained deeply relevant to professions, it has also developed as a distinct and diverse research industry in which it is common for aspiring general practitioners to highly specialised. Meanwhile, practitioners tend to be
1. Nine Bridges Golf Club in Yeoju.
Architects: Shingeru Ban. Modelling and Fabrication: Design-to-Production and
Blumer Lehmann. Photo: Blumer Lehmann.

2. Fab-Union robotic factory @ Ningjue Lyu. 3. Coreless Filament Winding. ๑ ICD/ITKE, University of Stuttgart.

4. Production of physical mockup
integrating modelling and feedback methods, by Tom Svilans and CITAstudio. involved as guest speakers with limited student contact, and typically engineering student projects are more likely to be theoretical than 'hands-on'. Engineering departments around the world are thus tasked with balancing the need for core education for an uncertain future with the need to advance high-quality pioneerin research that inevitably progresses into increasinger specialised areas, many with the potential to open up new professions and progress others.

With complementary aims, architecture in academia With complemente has evolved hough a diffent route whereby many schools are heavily a dulated with part-time staff who are active practitoners. Aspiing achitects are taught by an array of specialised researchers and tutors who draw from their experien based, where the ledglng practilionerlearns through synthesising simulaneous interests in design, technology, history and theory, practice management, project procurement and so on. Their understanding of the departments research activity is therefore set within the context of its proximity to their exposure to practice, albeit a rehearsal for practice at that. On this basis, it can be common in some universities to find research groups in both engineering and architecture departments with

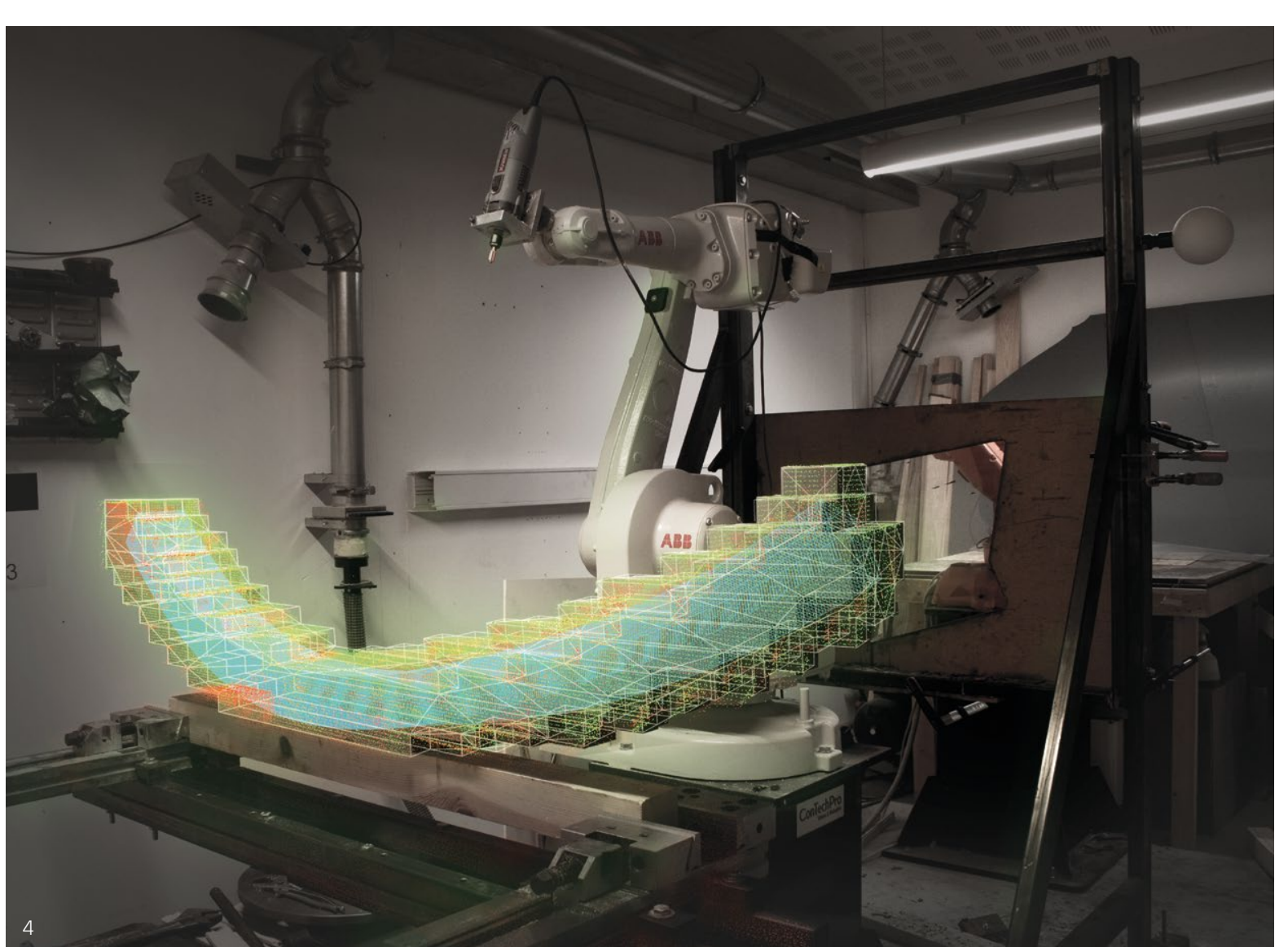

and structural design) yet operating entirely separately, as they are based on and designed for different traditions. While this may make sense in some cases locally, for those who have found ways to dissolve such silos the opportunities are extensive, and have never been more urgently required.

All contributions to this book come from and are representative of such collaborative trajectories. Few are troubled by labels or being constrained to a box and perhaps the most common reneration they relate to are those who, 200 or so years ago, speculat on where the future of such fundamental disciplines might go. "I recent years, and within the context of the disciplines of architecture and noneering, in both academic and professional senses we have witnessed innovative and productive convergence. Design is increasingy recognised as an expentise and a consideration that occurs within all aspects of architectural and engineering practice and is critical to both. Technical understandming and expertise, fuelled and aided by advances in computation, are equally fundamental to both domains. Programmes taught collaboratively between architecture and engineering departments are prolferating, and young university as their core motivation. Rather, they are more commonly inspired by what they will do as students, what they will be exposed to as learners and researchers, and what it all adds up to as a direction of travel. In short what is at stake is the challenge to improve both the future of life on the planet and the experience of living here in ways that planstainable for future generations, while harnessing the talent of complimentary disciplines toward that goal is surely our utmost priority.

The Digital Chain as Common Ground

Contemporary building culture stands before radical changes to its tha to respond to new requirements for energy efficiency, our coupcied wis the coupled with the energy crisis, we need to create clever solutions that enable smarter material use, higher energy conservation and better social and urban programmes while maintaining high architectural quality and cultura importance. The role of design is to develop solutions that engage the specific challenges of a given site, programme and environment. Until now, such creative 


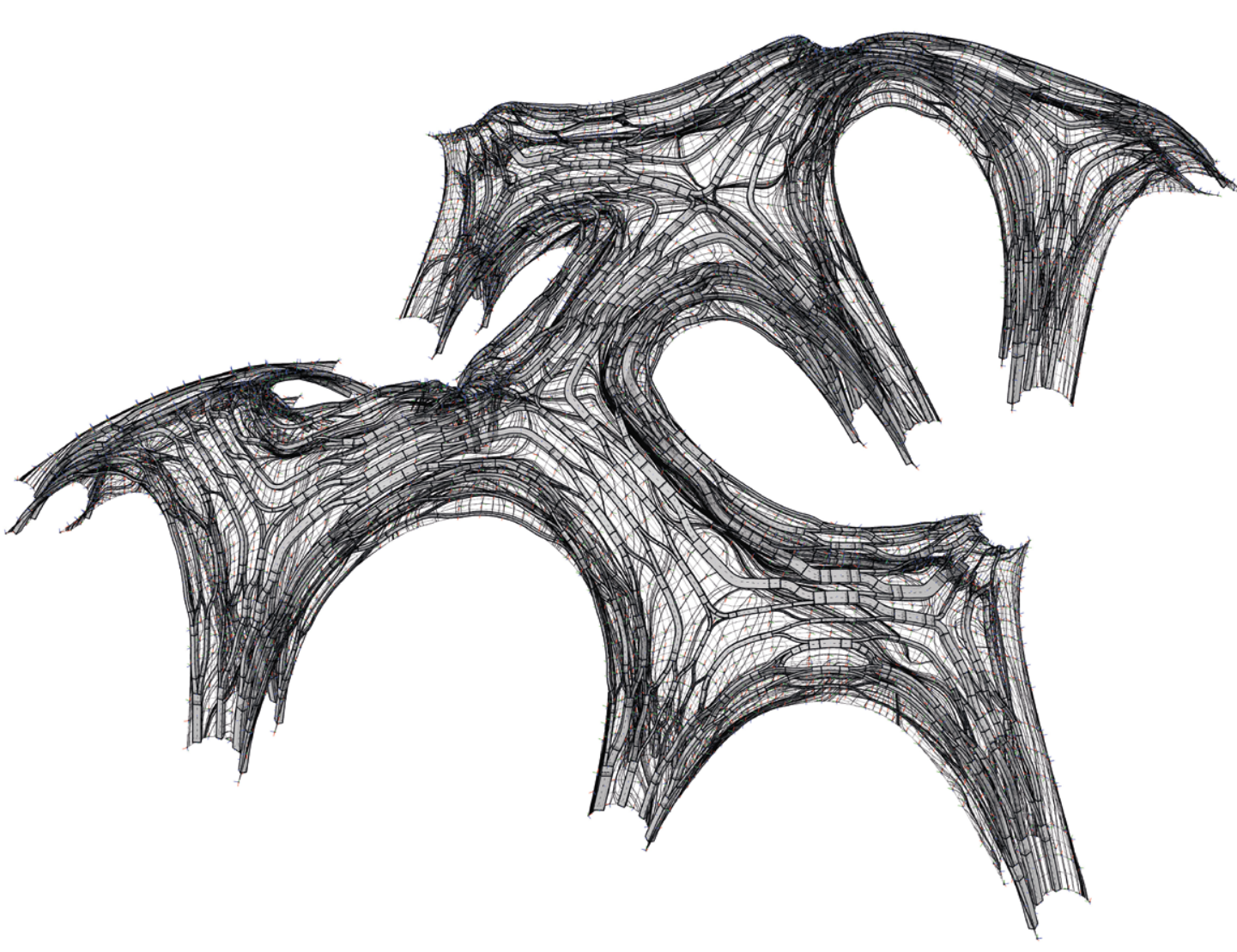

building practices of our modern industrialised building culture. However, with the increasing use of computational design strategies, contemporary buildin practice is endeavouring to develop new informationbased design models that fundamentally challenge the way we think, design and build architecture (Beesley et a. 2004). By establishing a digital chain, disital design to design fields enabling new interdisciplinary knowledge transfer. The ability to merge architectural design transter. The ability simulation of force and flow has influenced the thinking simulation of structural design, faclitating the reallsation of buildings with a higher degree of formal freedom and structural complexity. Sim

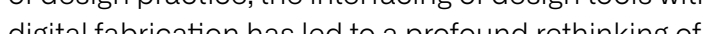
digltal fabrication has led to a profound rethinking of in prossice in architecture.

In professional practice, there is an acute awareness of these opportunities. While the field remains novel and methods and tools require maturing, the nascent understanding of the real design-based, technological, economic and environmental possibilities has led to emergence of a new professional research practice. in-house research environments to develop an understanding of the new tools and investigate how they can benefit from the interdisciplinary collaborations these enable. At the same time, a new cluster of research centres with a strong interdisciplinary focus has been instituted in leading academic environments. These are now examining how academic environments. These are now examining how computational logics can lead to a rethinking of the basic concepts that define architectu design practice, reconfiguil

Yet, where research cultures of architecture and - Yet, where research culures of arch communication engheorngare aware of each other, communicatior fragmented andlacks prolitic exchange. Scientific progress is urgently required to rethink current building design practice and bring together architects, engineers, design software developers and fabricators to identify design as a linear process of incremental refinement in which the project is passed between the difterent building partners is fundamentally challenged in this book. This volume shows how digital tools are being deployed to enable iterative thinking across processes of communication, simulation and materialisation, and asks how these three fundamental axes of design activity can challenge and change building practice. fragmented and lacks prolfic exchange. Scientific ovidigtal-materlal relations to reshape how we design and buld. Conventional thinking that positions proposal (2nd place) for the Tallinn

Architecture Biennale Installation
Competition, 2019, by Paul Poinet

and Tom Svilans.
Research Methodology and Approach

The work is interdisciplinary in two ways: first, as a hybrid of architectural and engineering practice; and second a three-way partnership between academia, practice and industry that also includes the fields of software development and innovative fabrication processes. Involving a relatively large and diverse number of academic and industry partners, it engages with multiple disciplines as well as multiple scales of enterprise. All the projects presented here share an experimental research-by-design methodology, focusing on design-led physical experimentation and full-scale prototyping. Such emphasis offers direct engagement with techniques and technologies all alon the digital chain, from design speculation and model analysis to specification and fabrication. This integrated approach positions research inquiries within a web of interconnected and living expertise and practice. Here, material experiments share the empirical dat that has been tested, analysed and evaluated by research teams across disciplines, ensuring results are appropriated and implemented in context. Physical experiments in design act as materia research inquiries (Duits, 2003; Beim and Ramsgaard research inquiries (Duits, 2003, Beim and Ramsgaard Thomsen 2011) by means of which the concepts and Design as a method of research inquiry is also a reflective practice in which the researcher engages in a dual mode of reflecting on and through action (Schön, 1983). Moving between scales and strategies of making Moving between scalo and strates of creates a conversation between he fragmented actions cretive motives of propostion and result. The method creative motives of proposition and result. The method is relevant for design-led research in architecture and engineering as it ins designcreativity and speculation to investigation. Building practice is often classified as a wicked problem in which the information needed to understand the problem depends on one's idea for solving it' (Aittel and Melvin, 1973). Design is therefore an active process by which the designer develops the dimensionality of a solution in the context of a given problem. Solutions are assessed not absolutely as or inferior. Employing a research-by-design methodology therefore allows the individual research project to

engage with the solution-led processes of creative . Here the work identifies three kinds of material evidence: speculative design probes generating ideation material prototyping enabling direct full-scale testing of defined design criteria against real-world methods of realisation; and demonstrators acting as proof-ofconcept testing design criteria in direct spatial contexts. All three kinds of evidence are seen as sequential, and iterative design phases build on the complexity of the project while addressing different contexts of research thinking. The research method places the exhibitions as central research instruments: beyond acting as a mean of public dissemination, they constitute milestones by which research results can be produced, tested and evaluated. The research method is known to the applicant and has been tested and evaluated in prio projects (Ramsgaard Thomsen and Tamke, 2009).

\section{Originality and Innovation}

The digitisation of architectural design tools has made a radical impact on building practice. The arrival of computer-aided design (CAD) tools during the 1970s chd 1980s and their maturing in the 1990s have resulted in a complete reconfiguration of architectural design practice (Kolarevic, 2003). Computer modelling in architectural design enables the description of variable eometries that calculate the values they embed

(Szalapaj, 2005), instigating new practices of algorithmic modelling that actively engage with information, directly calibrating and calculating the impact of a given design cheristion (Schwitter, 2005). Likewis, in the field of

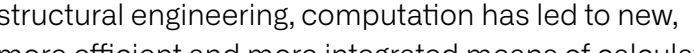
more efficient and more integrated means of calculating building performance. The digtisation of analysis toots and methods such as Finite Element (FE) analysis, which discretise complex problems into finite numbers of interrelated nodes to compute their force-relations, has revolutionised structural design and the buildings
that result from it (Clough and Wilson, 1999). 
At the same time, the interfacing of digital design tools design innovation, as it excludes our ability to understand with computer numerical control (CNC) fabrication has led to a profound rethinking of material practice in the building realm. Challenging the industriallst paradigm of mass production, practices such as file-to-factory, in which designers directly produce data that drive the CNC machines, and mass-customisation, in which repeatable elements with the same base morphology are differentiated (Scheurer, 2008), enable designers to realise complex structural solutions that optimise material use and therefore address issues of sustainability. Beyond this fundamental reshaping of the tools of our practices, digitisation has also impacted on the boundaries of our professions. By establishing a digital chain that brings together design, analysis, simulation, communication, specification and fabrication in a new integrated sequence (Kolarevic, 2005), building practice is promised the potential of stronger feedback between design phases, better interdisciplinary collaboration, smarter material usage and therefore better, more innovative and more creative design solutions (Mitchell, 2001). At present, the development of digital design tools in architecture is structured around large-scale industry-led efforts that have sought to standardise information and develop shared protocols between interdisciplinary partners (Ramsgaard Thomsen, 2016). However, core industry efforts in the form of building information modelling (BIM) with its overarching aims of unifying design information into a single shared model, remain incapable of tackling the inherent complexity and dynamic nature of building practice (Ramsgaard Thomsen 2016). The key constraints that limit the fulfilment of integrated digital capability and planning can be summarised as:

A disciplinary separation, whereby building practice Seen conceived as discrete desion phases in which the distinct partners (architects engineers, contractors and fabricators) hold separate design control and ren sibility associated with the respective phases. Where current practice retains this siloed understanding of the professions for both Itegal and practical reasons, the disital design chain as and practical reasons, the diftal design chan as presented here promises a fundamental rethinking of design methods and their associated tools as particular design methods and their associated tools as particu to each profession, he sector needs to develop new shared and interdisciplinary methods that cross the from the real potentials of the digital chain.

The impeding of design feedback. The design process is conventionally understood as a process of refinemen in which larger-scale problems are solved before smaller-scale ones. This understanding of design as a
progression through the scales limits the potential for how small-scale behaviours and complexities - be they material or detail - can affect large-scale concerns, such as the environment or structure. To support informed decision-making in the early design phase, Design Transactions: Rethinking Information Modelling for a New Material Age asserts that we need to develo mechanisms for multi-phased and multi-scalar feedback in which cyclical interdependencies can
be analysed and assessed.

A persistence of standardisation. Contemporary building practice relies on the industrialised manufacturing of standardised building materials that can be specified, that exist under legal codes and that ensure economic in building innovation as it limits the way in which we envisage the processes and materials of buildings. As new approaches employing advanced digital fabrication techniques such as robotics and 3D printing emerge, it becomes important to find new ways to integrate them with - and expand - current building practice.

What is required is a step change in the way that digital design tools are implemented in building practice. We urgently need to reconsider how these tools can inform a culture of collaboration and knowledge-sharing between disciplines. New understandings around how feedback in the design chain can be implemented in

meaningful and situated ways is required, and we need to rethink the foundations of the material cultures within which informed designs can be realised. This urgent shift is required to question the linearity of the conventional design chain and instead propose an iterative understanding of the design process, in which the three central concerns of communication, simulation and materialisation appear as recursively distributed and interdisciplinary activities By reconceiving the and chain in this way, we must allow for new kinds of intersections that merge concerns across the design chain and create opportunities for feedback between otherwise separate design phases.

Modelling Strategies and New Workflows

In the first of three core chapters, 'Modelling Strategies and New Workflows' includes contributions and and New Worktows includes contributions and ARUP, To to on ARUP, The Barlett School of Archilecture, BuroHappold Blumer-Lehmann, CITA at the Royal Danish Academy of Fine Arts Copenhagen, Design-to-Production, Fos + Partners, GXN Innovation, Institute for Advanced Architecture of Catalonia, Proving Ground, str.ucture, Shigeru Ban Architects, University of Applied Arts Vienn and Zaha Hadid Architects, among others. Across the respective presentations, the common trajectory is viability. However, this standardisation is a core restraint

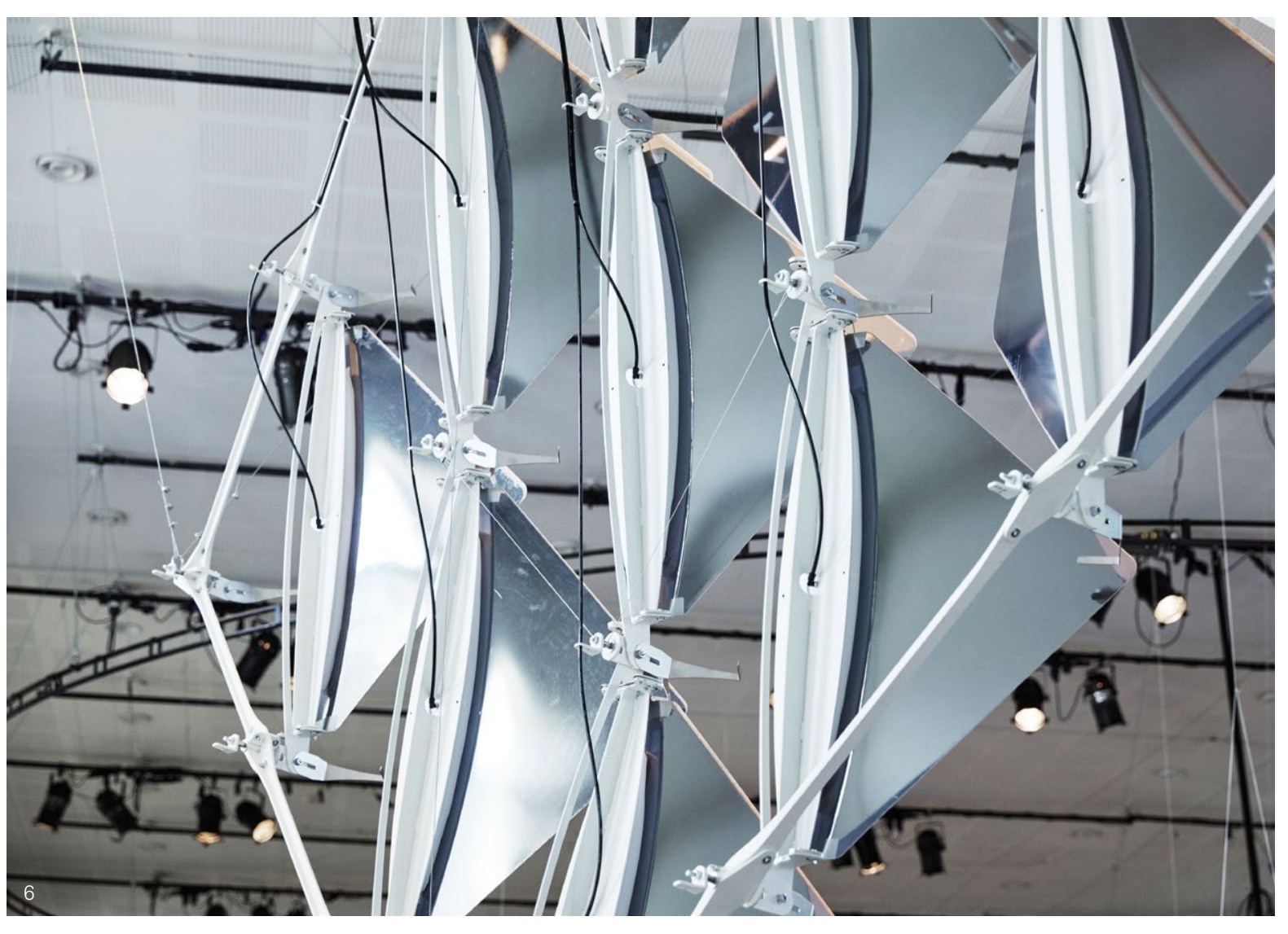

one that anticipates the imminent impact of machine learning on processes capable of managing inputs at far greater scale and speed than human-centric teams or processes to date. As the introduction outlines, the maintenance of dynamic datasets has shifted from preservation of geometric rule sets to the modelling of collaborative workflows, dispersed across multiple and simultaneous sources. The heated context for this field of research is ownership of, and responsibility for, data. s transactions occur, responsibilities transfer, and the built environment sector becomes fraught with debate on risk and liability. Consequently, physical output is less than half the story as for each manifestalion of a buitt component there are multiple digital records of its design-to-implementation pathway. What is emerging here is a call for open-source solutions, maintained through co-creation partnerships committed to investing the expertise of the user/adapter/designer within computation tooling - what Poinet and Fisher call a computational ecosystem. Such avenues would enable digital tooling to be tailored around projects and their particular objectives, and encourage an acceptance that software development expertise exists in abundance across industries and is not confined to software houses which seem bent on developing ever more generic software tooling.
6. Flectofold Demonstrator II by Saman
Saffarian, ITKE University of Stuttgart at Practice Futures - Buld ding Design for a
new Material Age Innochain exhibition

new Material Age Innochain exhibition,
Meldahl Smedie Exhibition Hall, KADK,
2018. Photo: Anders Ingvartsen 


\section{Design Integration}

Following on from these challenges, Chapter 2, 'Design Integration', includes contributions regarding ongoing research within Aalborg University, The Bartlett Scho of Architecture, UCL, BIG, BuroHappold, CITA at the Royal Danish Academy of Fine Arts Copenhagen, Foster + Partners, Institute for Advanced Architecture of Catalonia, Institute for Building Structures and Structur Design (ITKE) at the University of Stuttgart, KTH Royal Institute of Technology Stockholm, ROK, S-Form, Tongil University China, and White Arkitekter, among others. Central to the cumulative weight of these works is vehement conviction that design is an overarching practice and expertise that not only must prevail across all phases of procuring built environment projects, but has also reached an unprecedented position whereby such oversight and engagement are possible. One example of this shift is the prolific and rapid introduction of robotics into leading schools of architecture worldwide, where automated tooling is, in itself, of no new significance. What is significant, however is how robotics in design research and design practice challenges the designer to be engaged across the full spectrum of operations, from conceptual thinking, to creation of code, to material performance and material science, to tooling (effector) design, to production environment design, to the choreography of assembly and integration of feedback at every stage. In this sense, the contemporary design researcher is offered both the challenge and the means to occupy a position of influence where previously they were excluded - an opportunity to put theoretical skill and expertise into practice. What follows from this is a fundamental rethink regarding the destiny of graduates, one that may occupy a far broader scope than that of previous generations. Design research, whether developed in formal academic settings or industrial contexts, has escaped the narrow grasp of the professions and their silos, and offers new ground for harnessing the deply underutilised potentia of the construction industry.

\section{Novel Strategies for Materialisation}

Our third core chapter, 'Novel Strategies for

Materialisation', includes contributions referring to

ongoing research at AKT II, Arts et Métiers-ParisTech,
The Bartlett School of Architecture, UCL, BIG

BuroHappold, CITA at the Royal Danish Academy of Fine Arts Copenhagen, Foster + Partners, Henn, Institute for Advanced Architecture of Catalonia, Institute for Building Structures and Structural Design (ITKE) at the University of Stuttgart, Robots in Architecture, KTH Royal Institu of Technology Stockholm and XtreeE, among others. ine, design research as a prospective practice is laid bare as the agency for pioneering and specul creative invention. Vital exposure and access to advanced modelling and fabrication resources trigger an abundance of experimentation and prototyping. Just as any trading enterprise must calculate the return on investment value of capital expenditure, often over greater period of time than the potential redundancy of the acquired assets, likewise science-based research academies predominantly regard capital investment as serving a predetermined need to support research - that is, only acquiring the "right' tool for the job. Design research, however, often excels with the unexpected too , the unlikely process or the surprise result. Pioneering design research thrives when located within the midst and proximity of facilitating assets for which, at the critical early stages of research, there is often no known required application. Just as a modeller needs modelling software rich in capability, so too does a designer-maker need access to a wide variety of manufacturing processes. It is through such potential to speculate that the designer looks upon a robotic arm as a means to weave fibre into complex structural forms, or concrete printing as a means to create an artificial coral reef, or sets autonomous tooling the task of carving oak with the same dexterity and skill as a master maker - and even perhaps one specific maker, too.

Design Transactions: Rethinking Information Modelling for a New Material Age sets forth a challenge to both the construction industry and the academic community to deploy the abundance of capability, talent, and knowledge they share as an agency for collaborative transformation. The new work and, more importantly, the new partnerships that have emerged here not only new partnerships that urgency of taking a new direction. Offering sobering context to these arsuments is the daunting acceling of the global ecolo ef the has much to account for and rectify.
1. For example, The Royal Institute of British Arohtucts (RBBA) was founded in 1836, and Pour years later the first university-bas a established at King's College London, and a year later at UCL.

2. In his inaugural address (1841), Thomas Leverton Donaldson, UCL's first Professor
of Architecture, described architecture as wandering in a labyrinth of experiments'.

3. See, for example, the series of FABRICATE conferences and their associated publications:

Sheil, R. and Glynn, R. (eds.), 2011, Fabricate: : Making $\mathrm{D}$.
London, UCL Press.

Gramazio, F., Kohler, M. and Langenbers S. (eds.), 2014 Fabricate: Negotiating
Design \& Making, London, UCL Press.

Sheil, R., Glynn, R., Menges, A. and Skavara, M. (eds.), 2017, Fabricate:
Rethinking Design and Construction, London, UCL Press.

Sheil, R., Burry, J. and Sabin, J. (eds.),
2020, abricate: Design Meets Industy London, UCL Press.
Beesley, P., Cheng, N. and Williamson, R.S. (eds.), 2004, Fabrication: Examining the Digtal Practice of Architecture, Proceedings
of the $23 r d$ Annual Conference of the Architecture and the 2004 Conference of the AIA Technology in Architectural Practice

Beim, A. and Ramsgaard Thomsen, M.
(eds.), 2011, The Role of Material Evid. Architectural Research Daterial Evidence in Experiments, Copenhagen, Royal Danish Academy of Fine Arts.

Clough, R.W. and Wilson, E.L., 1999 in Proc. 5th US National Conference Computato

Duits, T., 2003, The Origin of Things: Sketches, Models and Prototypes

Kolarevic, B., 2003, 'Digital Morphogenesis' in Kolarevic, B. (ed.), Architecture in the Digital Age: Design and Manufacture Kolarevic, B., 2005, Information Master in the Digitalase: Design and Architecture London, Taylor \& Francis, p.58.

Mitchell, W.J., 2001, 'Roll Over Euclid: How Ragheb (ed) Frank Gehry Architect Now York, Solomon R. Guggenheim Foundation,
Ramsgaard Thomsen, M. 2016 'CComplex Proceedings of the 34 th Int, P. (eds.),

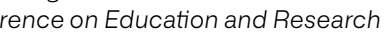
Computer Aided Architectural Design in

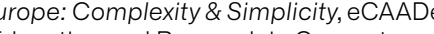
ided Architectural Design in Eurputer p.33-42.

Ramsgaard Thomsen, M. and Tamke, $\mathrm{M}$ Practice-Led Research in Architecture (conference paper) in Communicating (by) Design, International Conference on Design, Brussels, Belsium. Rittel, H. and Melvin W., 1973, 'Dilemmas in General Theory of Planning in Policy

Scheurer, F., 2008, 'Architectural CAD/CAM: Pushing (eds.), Manufacturing Material Effects: Rethinking Design and Making in Architecture, New York, Routledge,

Schön, D.A., 1983, The Reflective Practitioner: How Professionals Think

Schwitter, C., 2005, 'Engineering Complexity: Performance-Based Design (eds.), Performative B. and Malkawi, A. instrumentality, New York, Spon Press.

Szalapaj, P., 2005, Contemporary Process, Burlington, MA, Architectural Press (Elsevier). 


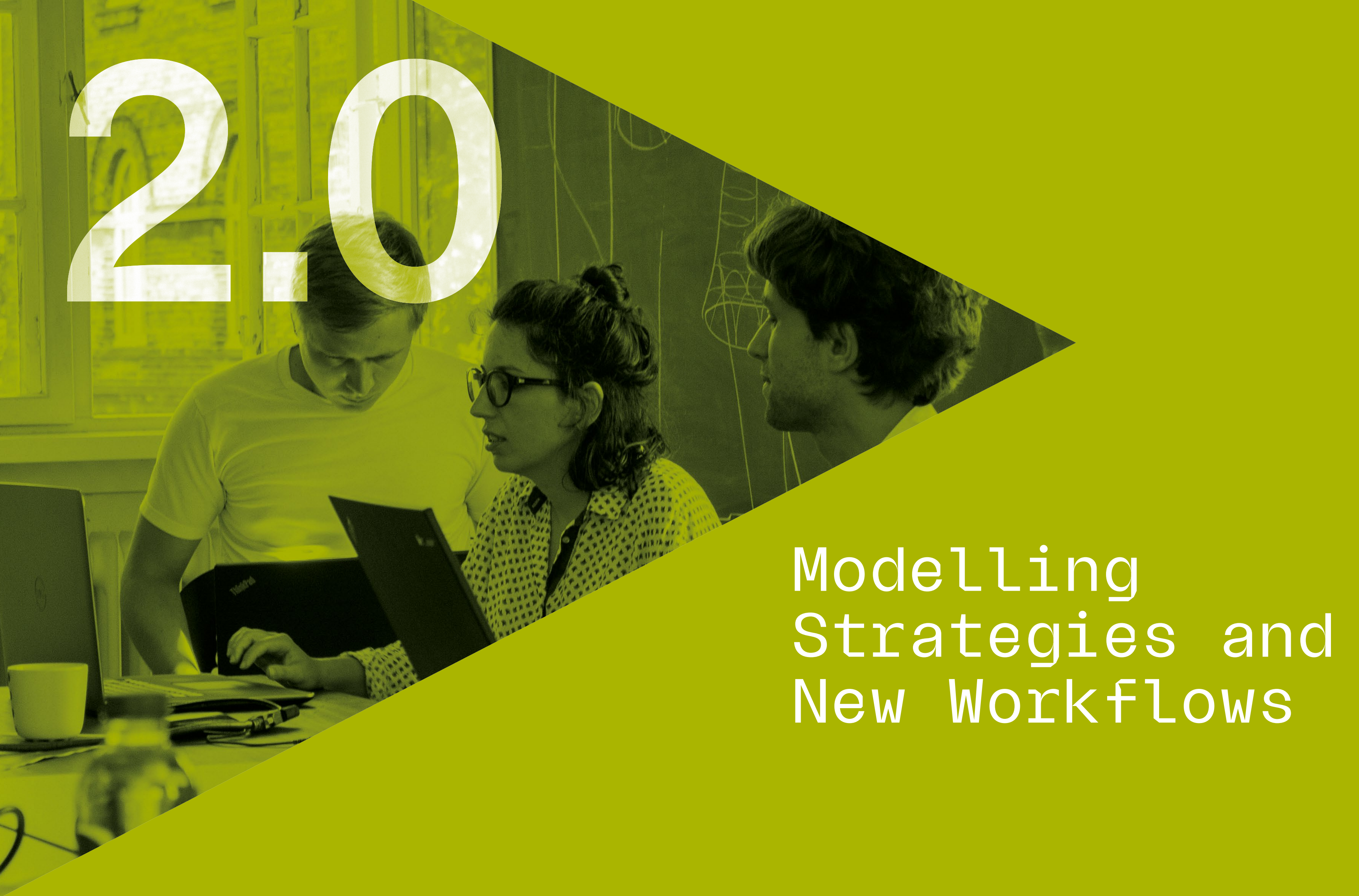




\section{New Paradigms for Digital \\ Prefabrication in Architecture}

Fabian Scheurer and Hanno Stehling

Design-to-Production

\section{Context}

In the early $21^{\text {st }}$ century, the building industry is facing some tough challenges. Ongoing urbanisation demands huge volumes of new housing and infrastructure in inner cities, but ageing societies can hardly provide the workforce to plan and build at the necessary pace (Farmer, 2016). In contrast to other economic sectors, where labour productivity has been growing for years and at astonishing rates (in Germany, for example, it has grown by $90 \%$ outside the construction industry since 1991), the architecture, engineering and construction (AEC) secto is dramatically lagging behind and has not yet managed to benefit substantially from the "digital revolution". A number of recent studies are urgently seeking a paradigm shift in the AEC industry worldwide - one that includes a turn toward prefabrication and industrialised processes, the subsequent front-loading of planning efforts and the seamless application of digital tools along the whole process chain increasing the vertical integration of the supply chain and the imp mentical in 'lean production princip's' (Rodrigues de Almidion

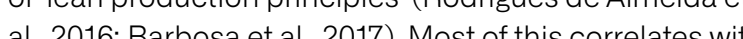
al., 2016, Barbosa etal, 2017). Most of this correlates with concepts such as In discuss discussed in industry since the 19o0s but have only rifting the shirting the planning effort forward, in an attempt to increase the impact and reduce the cost of decisions,
is shown in fig.1.

While fig.1 explains the chronological effects of front-loaded digttal design, the discussion about BIM and prefabrication in architecture has, until now, fallen short of addressing changes regarding the content the traditional onsite building process with industrialised prefabrication is leading to different requirements for the planning process as well. Merely shifting the planning efforts forward in time and answering the same questions earlier will not deliver the desired results but just lead to the same problems more quickly. Changing the building process requires the problem to be reframed and reordered in its entirety.

Sustainable Digital Prefabrication

In the wake of IPD and BIM, building with timber is preferred for a number of reasons. First, prefabrication has always been the default mode of operation in carpentry and, due to the weight of wood, is perfectly suited to the prefabrication of relatively large and highly-integrated building of relatively large and digital fabrication was an established technology in working with timber for years before planners fin illy started to adopt the BIM method: CNC-joinery machines are now ubiquitous, even in small-tomedium-sized carpentry firms. Third, and increasingly medium-sized carpenty firms. Third, and increasin extracting carbon dioxide from the atmo change, embelding tas wood in a building has a positive embedding it as wood in a building has a positive boundres have started to change in favour of boundaries have started to change in favour of largescale timber bulld ngs. Freeform timber projects like the Centre Pompidou Metz, La Seine Musicale or the recently-finished Swatch headquarters have changed public perception and paved the way toward orthogonal, pragmatic timber buildings. In this context, here we try to extract some findings from these significant projects and ask how digttal planning and production for
Timber columns, beams, slabs and wall elements can be detailed and pre-assembled offsite in the controlle environment of a factory. After a thorough quality check, the different elements are transported to the building site and 'snapped' together to erect a building. Typical most parts of such complex elements are produced using CNC machinery and are occasionally assembled by robots. The higher the degree of prefabrication, the more complexity is shifted from onsite installation to offsite assembly, which allows for more controlled processes and higher quality, but this improvement, in turn, brings new challenges. To avoid idle times, the whole chain - from ordering raw material to installatio onsite - needs to be orchestrated to guarantee delivery of the required components at a specific moment and in the desired place, resulting in significantly shorter building times. Since the time needed for installing prefabricated elements is typically shorter than the time needed for their pre-assembly, continuous workflows need to be organised by 'pulling' from the back-end rather than 'pushing' from the front of the process, following the so-called 'lean principle.' 2 To enable continuous installation onsite, the whole process needs to be synchronised or pre-assembly must build up stocks before installation starts. This complex supply chain management initially appears to add a lot of overhead costs, but we would argue that industrialised and controlled processes outweigh this effort with increased safety and reliability.

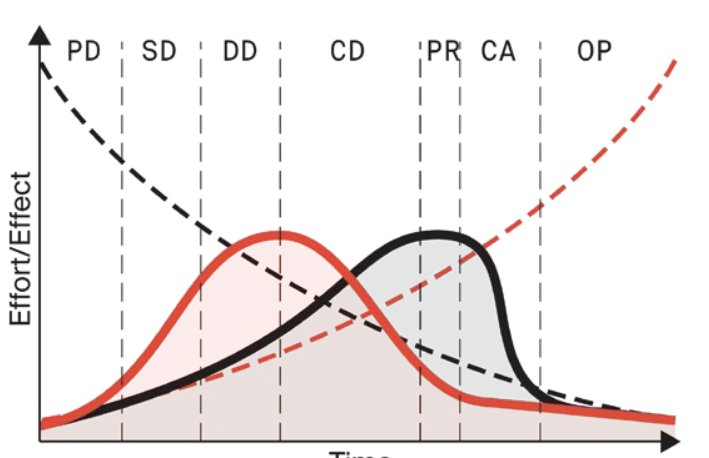

The traditional AEC approach of 'check dimensions nsite, then build' inevitably kills all attempts at lean, just-in-time, prefabrication. In such a reactive workflow, time-consuming production can only start once the preceding trade has left its measurable traces on the site. Parametric digital models allow quick adaptation to changing needs, but they cannot speed up the physical production processes, and, without real parallelisation, the main benefit of prefabrication is lost. In order for parts to fit into their designated locations, dimensions need to be defined and tolerances negotiated during the planning process, and checked throughout fabrication, assembly and installation. This requires a clear definition of responsibilities and interfaces between the different trades, not only on a process level but also within the digital building models that inform those processes. Abstract hull volumes can demarcate working spaces for different planners in a common reference model in the same way that interface definitions make possible the modular implementation of large-scale software projects. Today, digital building models are, however, mainly focused on building parts and not on the interfaces and connections between them. ${ }^{3}$ It could be argued that 'architectural tectonics', as defined by German architect Gottfried Semper (1803-79), have yet to attain the conceptual level of BIM.
- - - cost of design changes

- - - - effect of design changes traditional design process BIM design process 


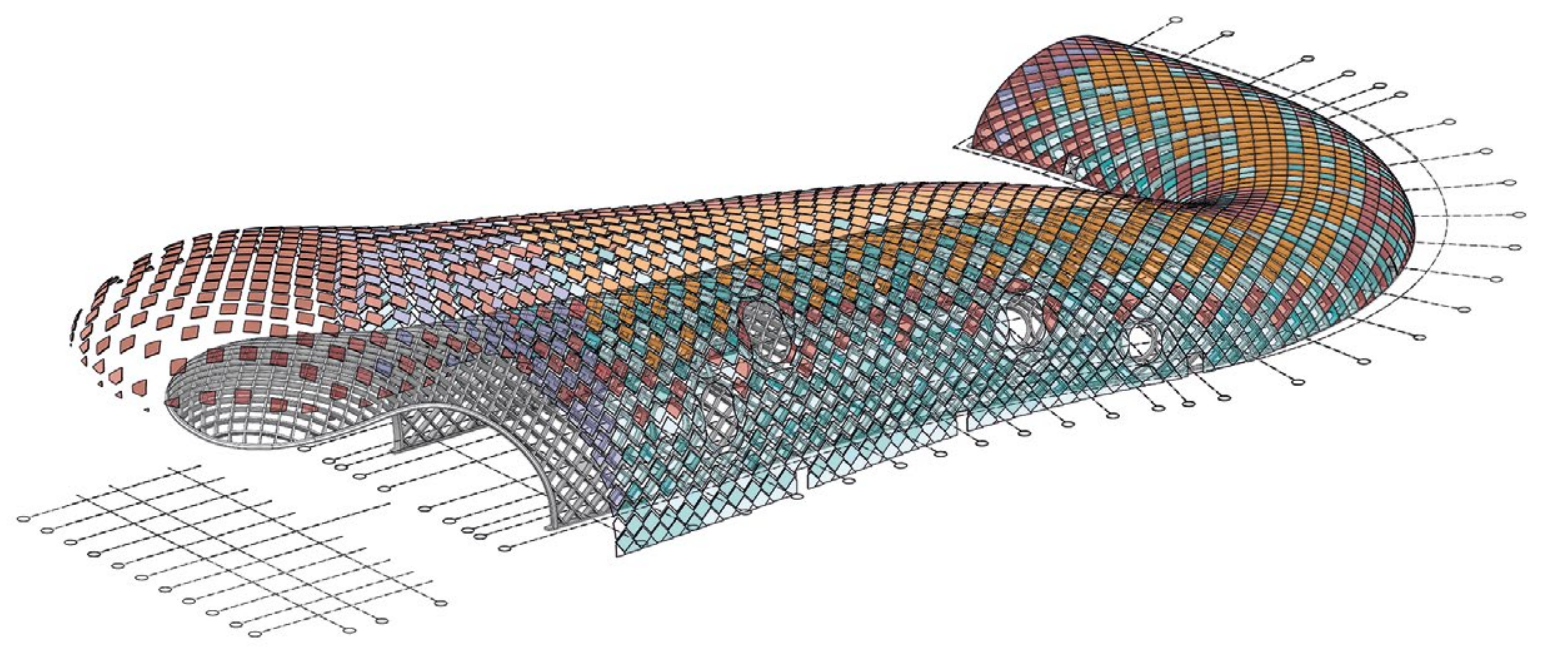

2

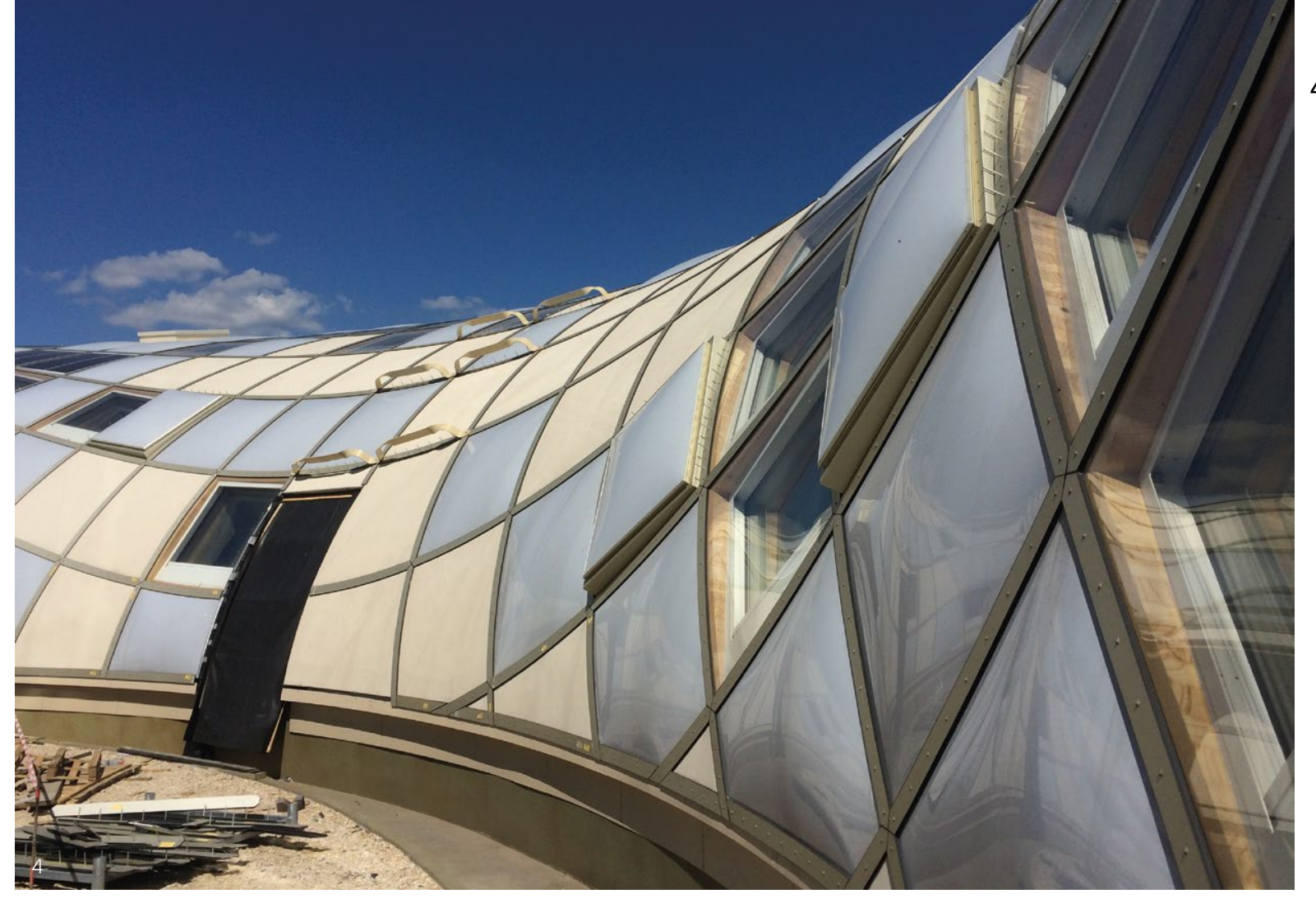

to be checked against manufacture and assembly, ideally in an automated fashion within the digital representation of the building. This requires connections to be modelled not just to simulate their structural performance (as is common in engineering) but also to describe their behaviour during assembly and installation. From which direction is a connection engaged and how does this influence the assembly sequence? How does the cost of fabricating the connection compare to the cost of assembly? How can that be optimised globally? The message of the Macheamy diagram becomes clear: design needs to take fabrication assembly seriously at an early stage of the process, to avoid unexpected delays and costs leter on In product design this is called 'Design for Manufacture and Assembly' (DFMA), and has been an established fie

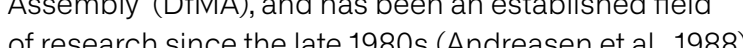
of research since the late 1080s (Andreasen et al., 1988). the fabictor the fabricator at the engineering stage of a project or after tender, wh th nochance to optimise the design. In future nduthes to be applied methodically at early stages, because the product design

BIM-to-Fabrication,

In Place of Shotgun Modelling

Even though more and more building projects are digitally planned, the biggest impediment to digital fabrication is still a lack of usable data. The main reaso
for this is a lack of focus. Building information models

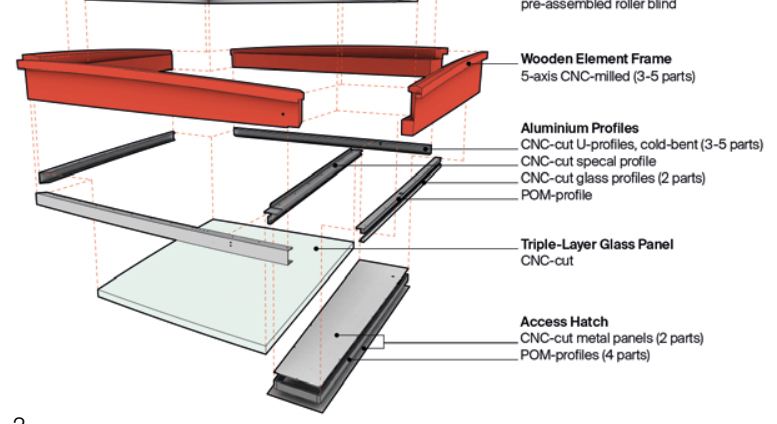

2. Swatch building coordination model. The geometric interface between the timber
structure and the façade was defined at the tender stage of the project by creating a coordination ports for air and electricity inlets and outlets were added to the hull models so that installation cut-outs in the timber structure could be coordinated with
mechanical, electrical and plumbing (MEP) mechanical, electrical and plumbing $(\mathrm{M}$ - $\mathrm{P})$
features and the respective connection points of the façade elements.

3\& 4. Swatch glass element, exploded and
installed. Nine different types of façade elements were developed as parametric 'products' so that 2,800 of them could be preassembled offsite and installed on the
timber structure. The most challenging were 470 closed-cavity glass elements with a cold-bent single glass panel on the outside, a geometrically-complex shading
mechanism and a triple-glass panelon mechanism and a triple-glass panel on
the inside, connected by a CNC-mille
wooden frame. are typically developed from front to back, driven by project development and design coordination but with limited knowledge about the fabrication environment. Without a clearly-defined objective, almost all planning models miss the target of being usable for production. Instead, fabricators start remodelling their digital mode from scratch when they finally become involved in the process. The phenomenon of dumping and remodelling information at every process stage has been described by Borrmann et al (2015).

Practical experience shows that digital information handed-down from previous planning stages is too handed-down from which parts of 'shotgun modes' are trustwothy, the pragmatic and safe approch is to throw the input away pragnatic and she an the continuous use of digital iformation throughout a multi-year planing accuracy and reliability.

Parametric Models: Accuracy and Reliability by Default

A typical timber CNC-machine works with a fabrication tolerance in the range of $0.5 \mathrm{~mm}$ and this accuracy is necessary to fabricate structurally-sound fitting details, e.g. slotted plates fixed to timber parts by steel dowels. digital model that is to directly control such a machine
must be at least as accurate as this, but expecting this level of precision at an early stage of the design process is unrealistic. Uncertainty and change will always be part of every planning process, resulting in multiple iterations. In this context models cannot be efficiently created with conventional 'manual' methods, but rather need to be generated based on parametric rules. This allows them to stay flexible and adapt to changing parameter values but, at the same time, prove highly accurate after each update. Creating such models requires a high degree systematisation to untangle and prioritise dependencies therefore, decisions need to be made about the underlying rules and structures. On the upside, due to this systematic approach, parametric models are to only accurate but are also reliable: the validity of the results depends more on the rules and in resuls de ands more or the rules and inputs than

Machine-Readable Models:

The Industrialisation of Planning

The main benefit of working with digital models is their 'machine-readability' Instead of needing an experienced 'machine-readability'. Instead of needing an experienced human to interpreta plan drawng, diglal models can be read, checked and manipulated by algorithms. Models themselves can serve as input for new models, which is the core feature of the productivity leap expected from digitalisation. But when high levels of responsibility and risk are involved - like in the AEC industry - all digital tools we currently use rely on well-structured data. Eve 4 


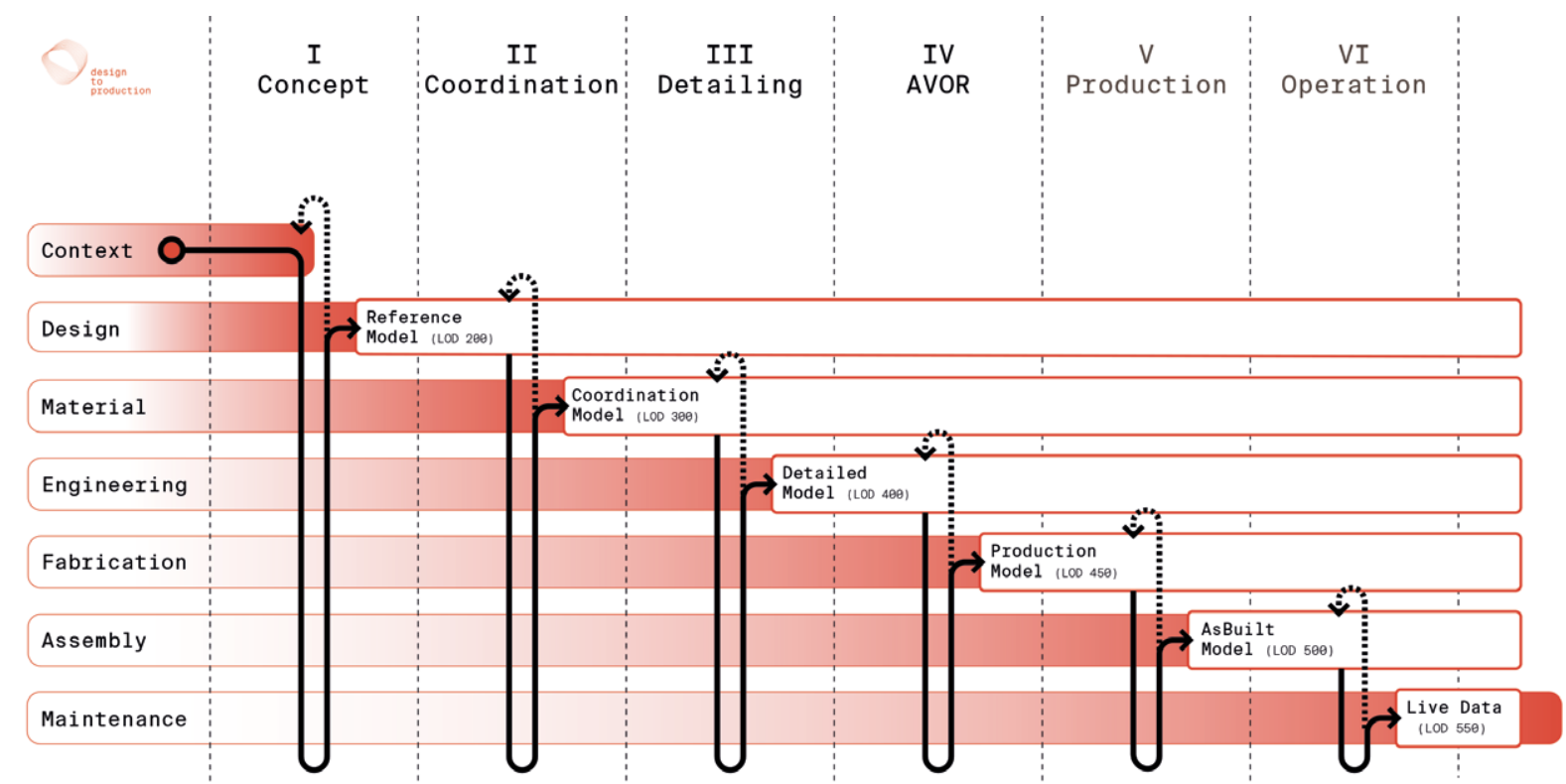

artificial intelligence has made some progress in recent years, vague or contradicting building instructions are still dangerous. To unambiguously encode information about a complex building into a set of digital models used by multiple parties for very different purposes, a myriad of very precise agreements are necessary, regarding ontologies of model objects, naming conventions, types and so on. Standardised data formats such as IFC are helping with this, but still leave a lot of work and responsibility for BIM management. a profession still in its formation phase.

Small is Beautiful:

Multiple Minimal Models

By definition, a model is an abstract description of a part of the real world. A manageable digital model is one that contains as little information as necessary to serve its purpose, without creating redundancies and inconsistencies. The decisive and recuringauestion during the process of modelling is: What information from the rocess of modellinglisinatinformation The anser world The answer depends on its purpose, which leads to a needs to be cleauences. First, the purpose of a model needs to be clearly defined (see 'shotgun models', as ment be med earlier). Second, for diferent purposes there will be multiple models of a building that cannot be integrated into one single model without violating the minimum rule. When multiple models are used to describe the same building from different points of view they need to reference each other or a common base model to avoid inconsistencies, otherwise it cannot be
guaranteed that they do not actually describe different
5. Agile Design-to-Production process (from left to right) and varying abstraction

6. Reference models for the Swatc building, including grid, surface and beam axis. It includes a naming and
numbering concept for all beams and façade elements from the beginning facade elements from the beginning
of the project until the end, which has
remained unchanged for five years. process through different stages in time buildings. What BIM needs is a modelling environment on an organisational and a technological level that and safe fashion.

\section{Multi-Scalar Models}

On an organisational level, planning processes in the AEC industry often follow the so-called 'waterfall model', Starting at a conceptual level and progressing to the production stage, increasingly 'concrete' topics are investigated, such as materiality, structure, fabrication, assembly and maintenance, while integrating larger amounts of information into more detailed data models. Neglecting practical questions for the first two or three rounds of planning is obviously not compatible with the requirements of DFMA, but on closer inspection there is also a modelling problem. In the waterfall approach, the model with a higher level of detail (LOD) typically replaces the less-detailed version. The aim of parametric modelling is to automatically generate the next level of detail from the previous model, but this becomes impossible once the most important input - the lower-detail model - has been declared 'outdated'. To automate modelling, we not only need to handle multiple models for different purposes but also for multiple levels of scale or abstraction.

\section{Design to Production and Back}

A different process model has been developed after more than a decade of experience in freeform timber projects and continuous discussions on the aforementioned modelling conundrums.

All topics, from the abstract context to concrete fabrication, assembly and maintenance, are addressed from the outset in an 'agile' fashion to provide a viable solution at every stage. This requires enough knowle solution at evert stage. This requies enough knowledge design regarding smart integrated opportunities, either by tapping into the know-how of production specialists at ealy poject hases or by developing and following at early proct stages or by deveping and following To efficienty arive at a high-audity model the To efficiently arrive at a high-quality model, the volume or formation needs to be ke mo to minimum. Only the decisions made and justifed at any given stage need to be reflected in the data, thus the process 're In add ton, the 'abstract' models are not replaced by the more 'detailed model at the next stage, but instead are This alive and extended by the additional information. This requires updates to be made to the abstract model in conjunction with later findings, while therefore the probability of change at later stages should also be ddressed before adding to any model. 
The aforementioned topics are crucial for utilising the potential of digitalisation within the AEC industry. We have been wrestling with, and partly solving, issues in experimental fashion. Today, it is exciling to see such discussions happening at a much larger scale, namely around standard 'orthogonal' buildings.

Prefabrication is slowly gaining traction but is still an 'alien' concept in many parts of the AEC industry, particularly as it does not fit many of the traditional

approaches for scheduling, the handling of tolerances and the development of details. BIM is becoming more of digital data models needs to be extended all the way from design to digital fabrication and assembly processes. The modelling paradigms of BIM are largely concerned with building components but do not define interfaces and connections. Multi-scalar parametric modelling is not yet implemented, either in the workflows, tools or standards. non-standard 'curvy' projects since 2007, in a somewh common but the continuous use and coordination

outlook

Prefabricated timber provides a chance to finally accomplish the digital turn in AEC with real industrialised processes and a productivity that catches up with that of other fields. But to successfully exploit the already existing digital production facilities at the end of the process, the planning at the start needs to focus on what is needed for an industrialised, lean production of buildings - and to come up with new paradigms to digitally model those processes.
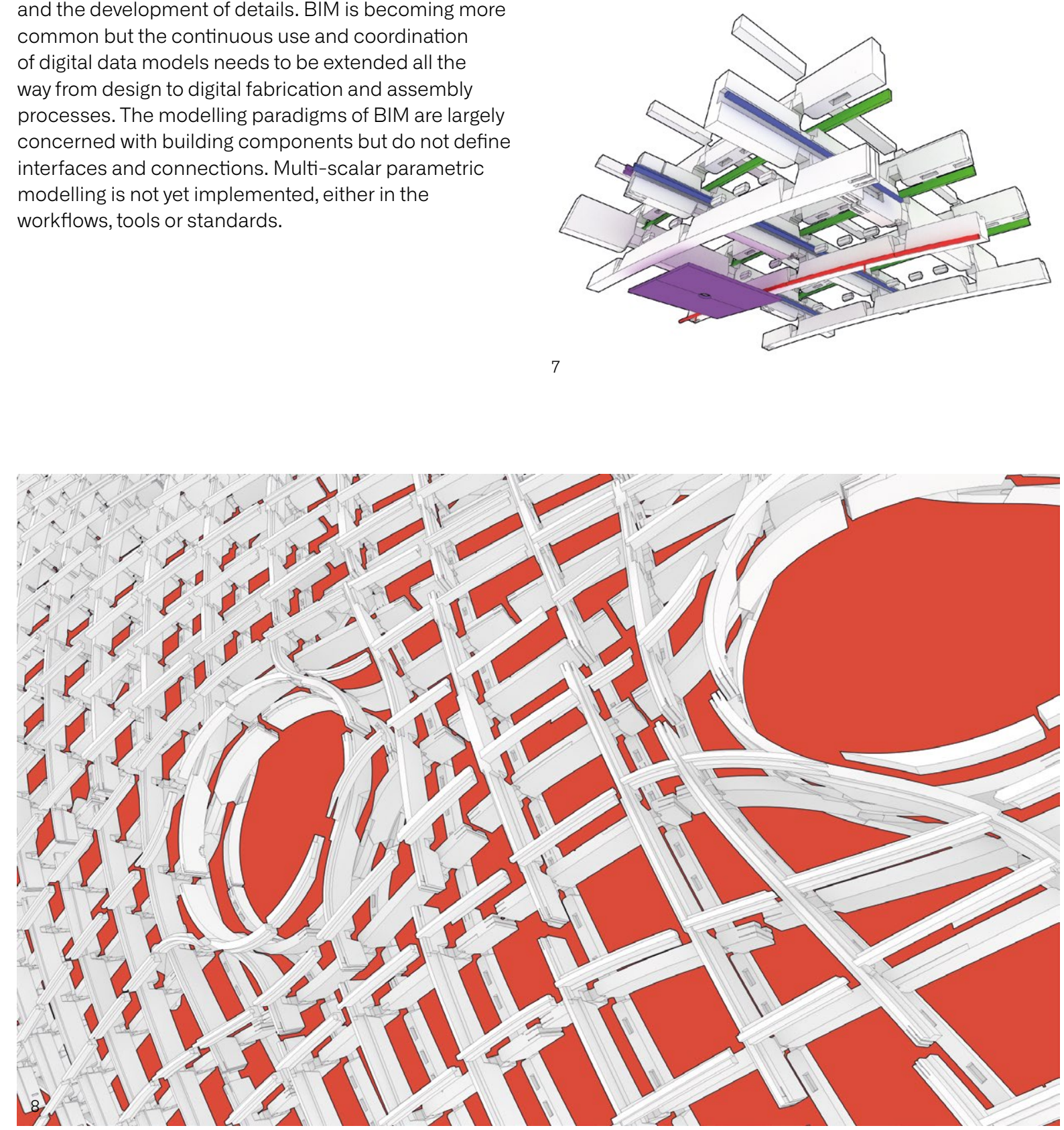

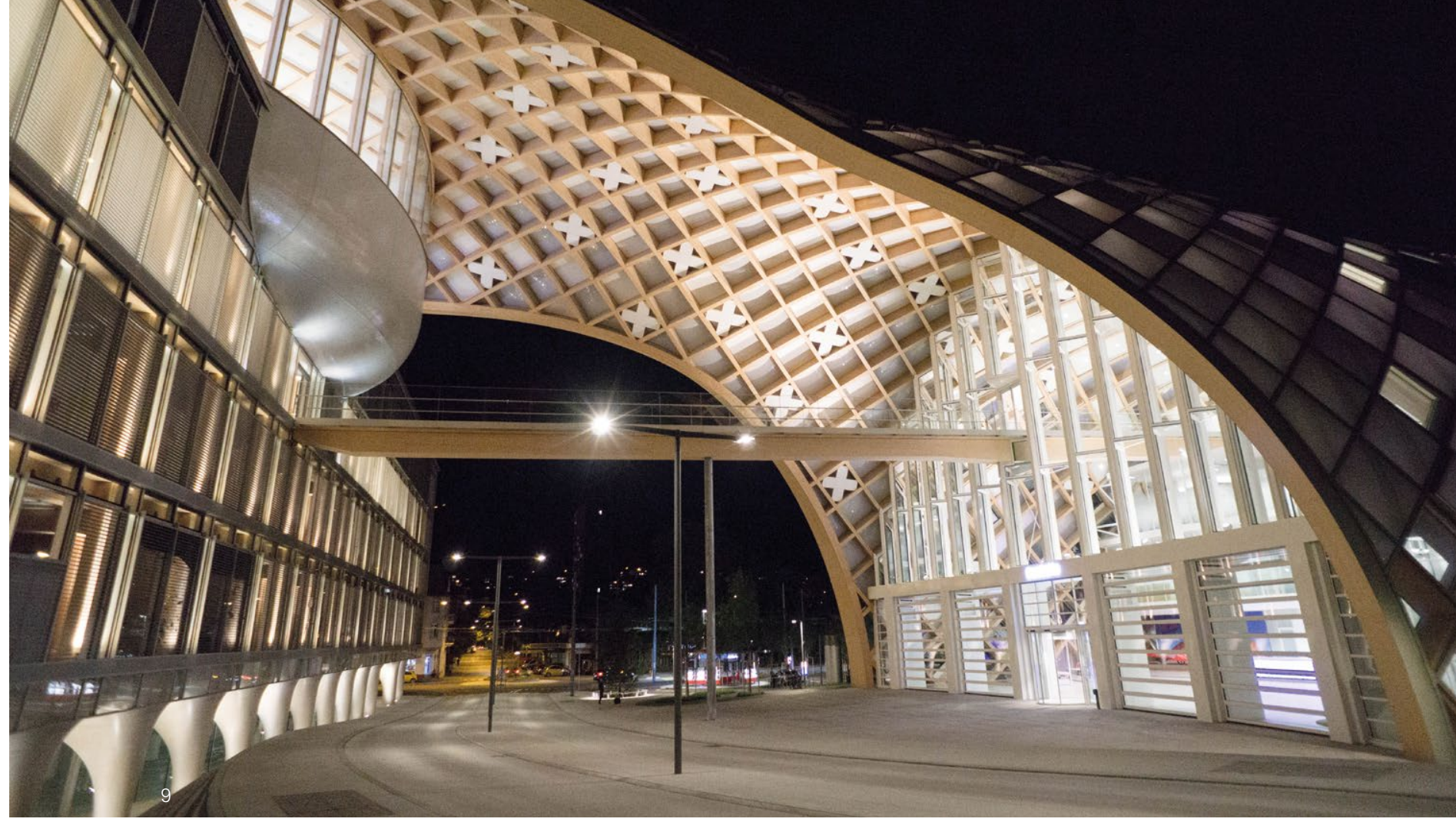

7. Detailed model of four fields, showing building parts close to their final physical
orm. Due to the increased amount of data, detailed models are often subdivided according to assembly sectors, componen
types or other criteria. Given that all parts respect the boundaries defined by the preceding coordination model, interfaces
between different detailed models can be between different
kept to a minimum.

8. Explosion Drawing of detailed timber

structure.

9. Swatch headquarters. The timber structure and façade comprise approximately 75,000 bespoke components, prefabricated in
more than 20 locations. Most of these components were preassembled offsite components were preassembled fifsite
into 4,600 beam segments and 2,800 facade elements and were then delivered
to site immediately before their installatio

Bibliography

Andreasen, M., Kahler, S., Lund, T. and Switt,
K.,1988, Design for Assembly. New York.

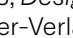

Barbosa, F., Woetzel, J., Mischke, J, Ribeirnno, M.J., Sridhar, M., Parsons, M., Bertram, N. and Brown, S., 2017, ( (n ey Global Institute.

Borrmann, A., König, M., Koch, C. and Modeling, Wiesbaden, Springer.

buildingSMART International Ltd., 2019, IFC/RELEASE/IFC4_-1/FINAL/HTML

Davis, D. 2011, The MacLeamy Curve (blos entry). www.danieldavis.com/macleamy (accessed 10 December 2019).

Farmer, M., 2016, The Farmer Review of the UK Construction Labour Model, London,
Construction Leadership Council (CLC). www.cast-consultancy.com/news-casts model-3 (accessed 10 December 2019).
Rodrigues de Almeida, P., Gerbert, P., Castagnino. S. and Rothballer. C., 2016 Shaping the Future of Construction Introduction, Geneva, World Economic Shaping the Future_of Construction.pof (accessed 10 December 2019).

Semper, G. 1860, Der Stili in den technischen und tektonischen Künsten,
Frankfurt a.M. Verlag für Kunst und Wiss.

Statistisches Bundesamt, 2019, Fachserie 18, Reihe 1.5, Tabelle 2.14. https:///www. Environment/Sustainable-Development( (accessed 10 February 2020).

Notes

Statistisches Bundesamt, 2017. 2. www.lean.org/whatslean/principles.cfm 3. The BIM standard IFCX4 (Industry subclasses of IfcElement for defining components that make up an AEC (a) to define 'connectivity between elements'. Furthermore, IFC-connections cannot be parameters (buildingSMART International Ltd, 2019). 


\section{Information-Rich Exploration in the Early Design Phase}

\section{Zeynep Aksöz}

University of Applied Arts, Vienna

\section{Introduction}

As computational analysis accelerates in visualising performance objectives, the integration of generative design and metaheuristic search tools, which are designed for systematic problem-solving, is shifting to the early stages of the design process (Harding and Shepherd, 2014). The strength of these tools in

generating and evaluating design solutions offers a new perspective on the conceptualisation phase, where it is approached as a problem-solving process.

The first requirement for successful problem-solvin is a clear and rational definition of the problem that is to be solved. This calls for clearly-defined variables and goals, addressing all aspects of the problem, hence the problem becomes a multi objective optimisation

problem. In conventional problem-solving processes for optimisation metaheuristic solvers iteratively renerate and evaluate different solutions, searching for optimal solutions that address all the selected goals. However in working with multiple objectives, the selected goals can be in conflict This means that to inproved the objectivescan resultin areduction of the other objectives. Consequenty, the 'optima solutions' discoved by the solver satisfy al the goals equaly, discovered by the solver satisfy all the goals equally, Design, however, is a process of dealing with situations involving uncertainty, uniqueness and situations involving uncertainty, uniqueness and contict (Fischer et al., 1991). The early design process is evolutionary, by its very nature. Through continuous evaluation and reflection, concepts are revised, reframed or completely discarded (Mothersill and Bove, 2017). Early design is thus an ambiguous phase, Where there are no definitive formulations and no
For successful problem-formulation, a complete Un needs to be outlined. As design is a linear process, the information gathered in the later stages of design development typically vanishes with each new project. By contrast, the technologies employed in the scope of this research utilise previous experiences, harvesting information gathered in the later stages of design and integrating it into subsequent early phases, to establis information-rich environments using the reoccurring routine processes involved in design.

This research aims to bring a new perspective to the early design process, where creative exploration emerges out of a collaboration between designer and computer. The following sections explain the processes developed within the scope of a project in collaboration with structural design specialists str.ucture. Existing an newly developed methods are evaluated for their

accessibility and usability within the design environment, as well as the reliability of these tools in real-life projects.
Case Study: Triangulated

Façade for a Car Park

Using the industry collaboration with str.ucture as a angible example, the benefits and limitations of a work flow using generative design are outlined here. The problem was to design a triangulated textile façade that would function as the skin of an existing concrete structure for a car park. The triangulated structure was to be designed in consideration of the geometric limitations of a steel form-fit connection, which was previously developed as a façade detail by str.ucture and Design-to-Production. The joining system consisted of form-fit cog connections on a circular laser-cut steel plate. As the façade design was

dependent on the system's geometric limitations, it was essential to develop a design workflow that accommodated the freedom of early exploration, while generating geometries that remained within the fabrication domain.
Here, understanding the design problem is equal o solving it (Rittel, 1972). The role of the designer is therefore, not only to find the correct solution but to find the right question to ask: finding the correct design variables and design goals to proceed with generative exploration is an exploratory process in itself.

In optimisation, aspects of a problem are incorporated to create one or more measures of effectiveness, which ultimately become the criteria by which design solutions are evaluated. Here, the decision-making process is informed by analysis and simulation, motivating designers to define and evaluate goals early on. With this approach comes particular obsession with numbers and quantitios, where qualitative aspects can easily be mixed with quantitative performance (Harding and Shepherd, 2014). The integration of metaneuristic solvers in earlier 2014). of design can help the designer to explore multiple solutions. However, this exploration only takes place in the realm of quantifiab which aspects The objective of this research project is to explo The objective of this research projectis to exp and ded to suited to the early design process. Creative exploration is the plo as an and the design goals are mutable and thid. The research, therefore, gnestigates processes that can accommodate ambiguty and imprecision, while providing the designer wh a playfur environment through which to develop the conceptual framework of the design problem. Instead of avoiding metaheuristics and the intelligent processes offered by advancing computational power, the research ullises the benefits of these technologies to establish new processes th

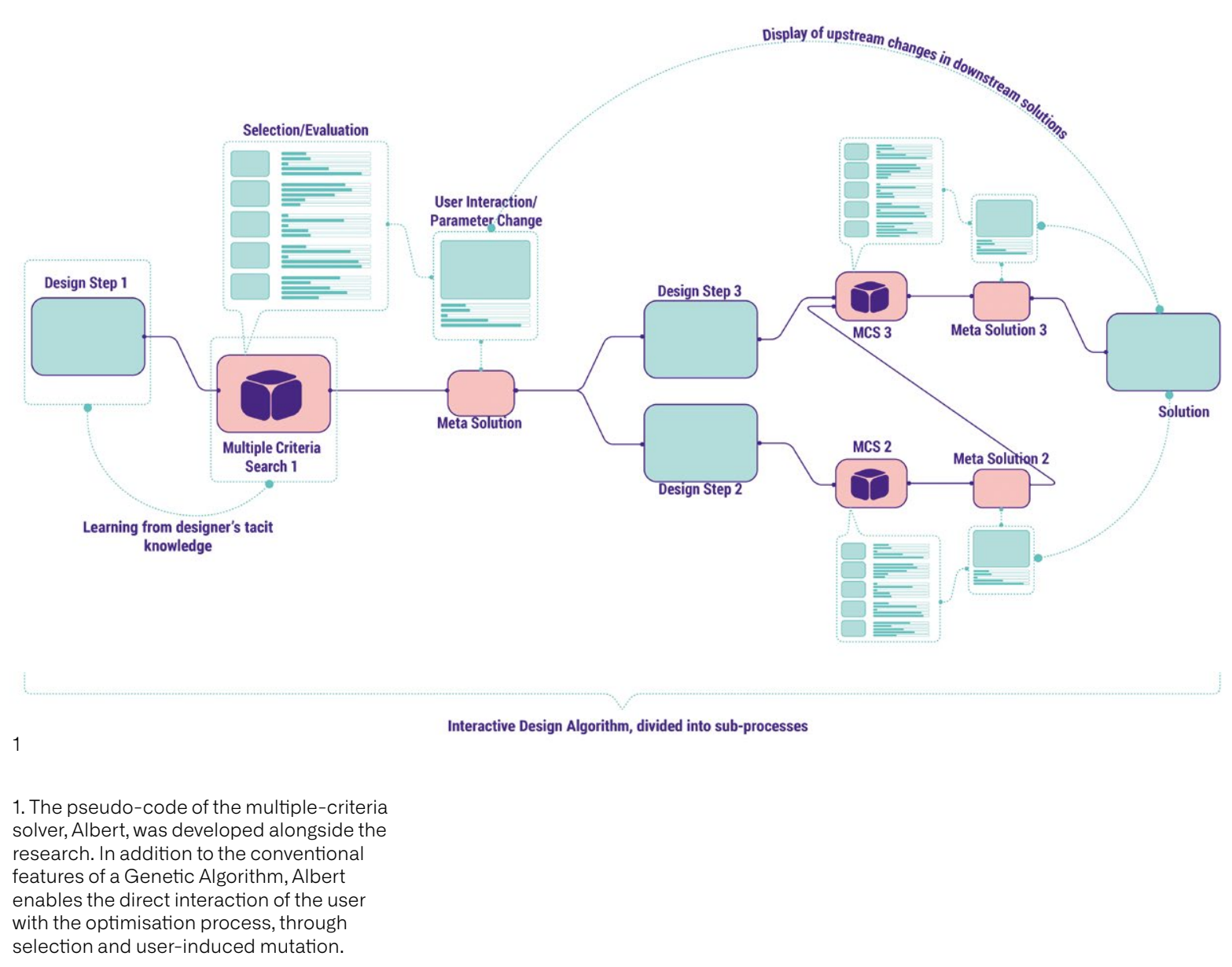



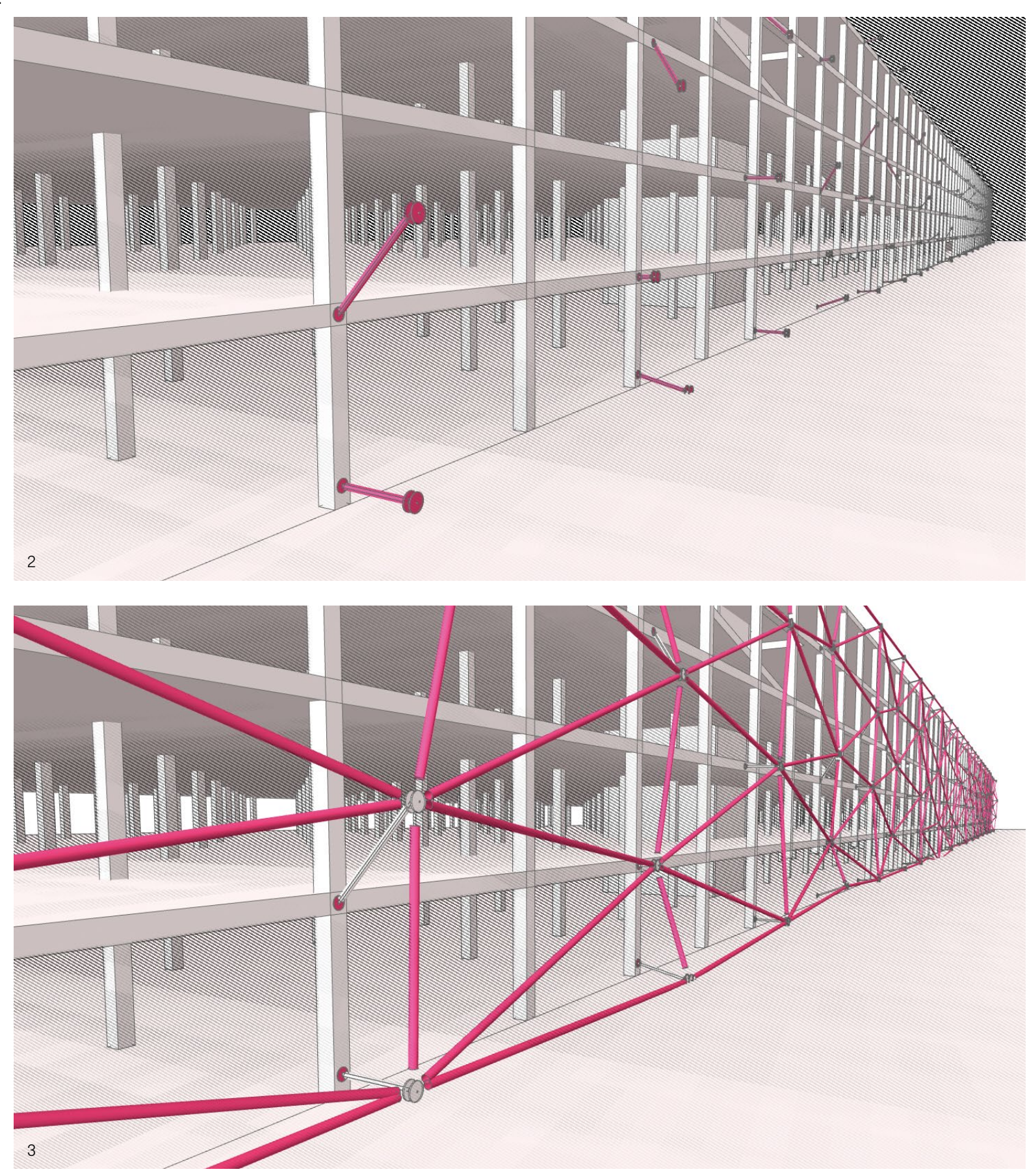

2\& 3. The façade consists of a form-fit cog joining system that connects triangular phases of the system, where the joints are installed on the existing construction of the building and the triangle panels are installed

4. The pre-defined constraints of
the design problem.

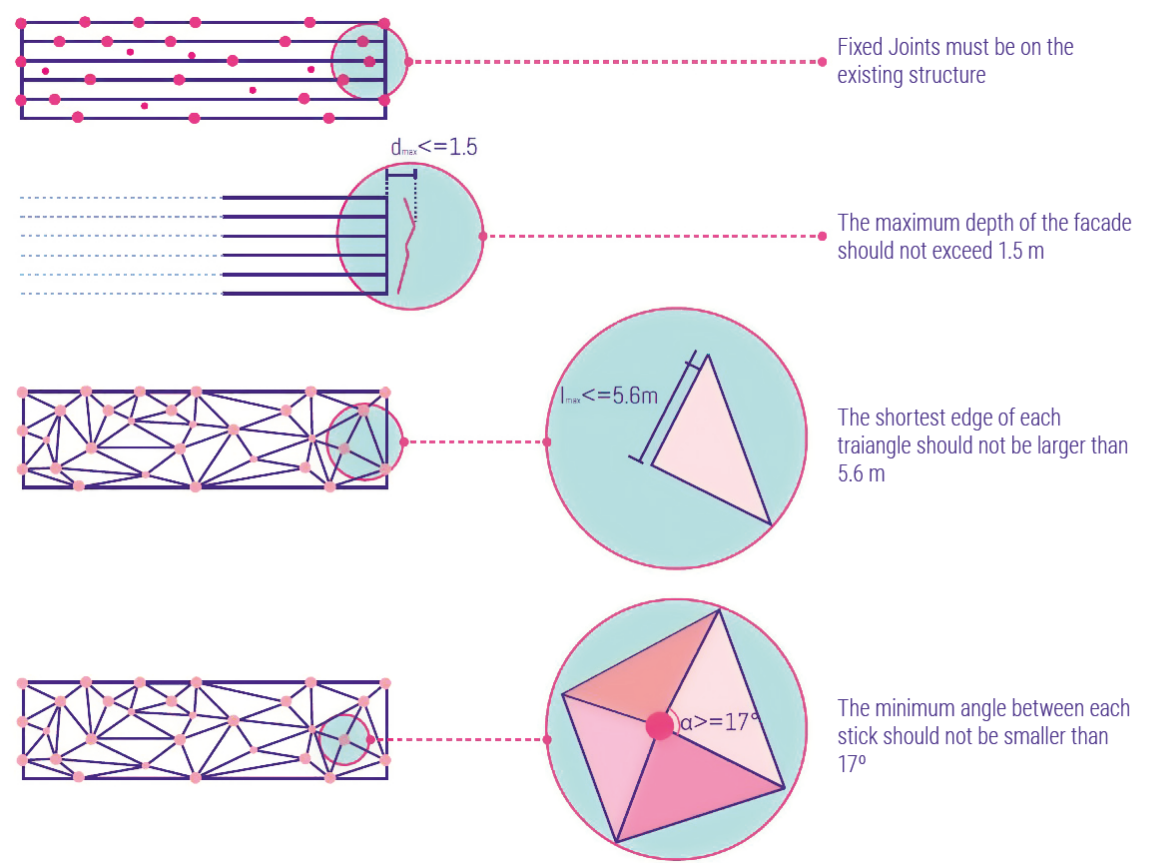

The flexibility of the laser-cutting process allowed the façade system to accommodate differentiated triangles, although a minimum angle between the elements had to be considered to avoid collisions. Another limitation was defined by the membrane fabricator, regarding the length of the edges, which could not exceed the maximum cut-edge of the fabric roll. The existing structure of the car park was used as a framework onto which to attach the facade. Some joints, therefore, had to be installed on the existing construction, while the facade depth could the existing $1.5 \mathrm{~m}$ (fis 3). The objectives were defined not exceed $1.5 m$ (fis). The objics stiffness and cost-effectiveness of the system. Although this optimisation problem seems clealy defined through the given fabrication constraints, the defined parametrisation process, to select appropriate design variables and objectives, also involved lengthy exploration and evaluation of the different methods of problem formulation. It was essential to find a set of parameters that would successfully control the top-down global geometyand bortom-up sizng of for probs simultan problem, simultaneously. Finally, he optimisation process would require us to optimise the size of the elements and the angles between them, and to generate geometries that met the aesthetic aims of the team, which cannot be measured in the design code itself. Different methods of parametrisation were tested

optimisation problem was, consequently, divided into two sections. A set of variables controlled the global geometry of the façade by varying the frequency and amplitude of four sine curves along the floorplates of the existing structure, generating a doubly-curved surface. Another set of variables controlled the number of pieces and locations of the joints on the surface, within the limitations of the existing construction. The joints were added as vertices that defined the triangulated geometry using the Delaunay triangulation method (Gärtner and Hoffmann, 2013) (fig. 4). Using a particle springs system, a method for structural form finding where a collection a mont or structural form finding, where a collection Kangaroo (Piker, 2013), the ideal location for each join was defined using the given fabrication constraints. Due to conflicting criteria, however, Kangaroo counts. Due to Dot in A heuristic method wa in A heuristic mont Ala

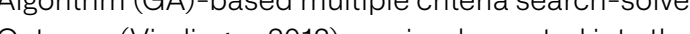
Octopus (VAeringer, 2013) was implamented into the work heuristics with a constraint-based solver, diferent globa geometries could be iteratively generated by GA. While Kangaroo was controlling he constraints, the generated solutions were evaluated by GA, measuring the fallures

Ontring in the system regarding fabrication constraints. One of the major components in the evaluation of the selected problem-formulation is visual access to the

53 

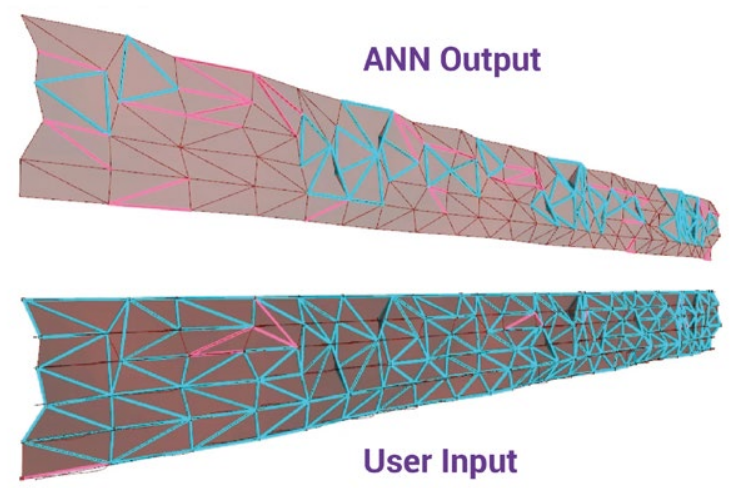

in a convergence toward mathematically 'good' solution that are physically impossible to fabricate. The reason for this lies in the multiple-criteria nature of the problem, where each objective is equally unsatisfied. The Pareto front displays a set of 'optimum solutions' for selected objectives that discard geometric constraints to satisfy other criteria. A lack of visual representation and user interaction with the search process results in the establishment of solutions that appear to meet the quantitative goals, yet these solutions are completely irrational. For example, in one case the solver succeeded in avoiding any large structural members (in this case, rods) and any acute angles between them, while it minimised the cost by not generating any façade components. This indicates two problems: first, the parameters and objectives are ill-defined and, second the designer has access to the solutions too late to be able to change the selected problem formulation. Ultimately, this process is overly time-consuming and is therefore usually avoided in the early design phase.

In this context the performance of the platform is not the only issue; the manner in which a playful and intuitive design approach vanishes with the integration of generative tools is also of concern. Do we have to hand over our decisions to the computer to discover novel solution Achieving novelty through senerative search solutions? Achieving tovelty through generative search seems persusine buther quantitative goals, which are usually related to quantitative goals, which are usually related to perfornance. Evaluating design solutions solely by considering quantifable goals disregards the vital qualtative aspects of the design process, since these cannot be measured by any metric. Yet the conceptual idea of design is more than its quantitative performance the aesthetic judgement of each designer must also be considered. Conversely, sometimes the aesthetic quality becomes so important that the quantitative goals can be completely overlooked. Hence, the early creative replacing creative exploration with generative desig these tools should become an amplification of the creative process, giving the designer the scope to intervene and change the selected parametric setup along the way. Thus, we should use generative design as an extension of creative exploration, and approach these methods as tools of communication rather than decision-making tools. Accordingly, they should help us to formulate questions rather than find answers. Albert - a multiple-criteria search tool developed during the scope of the research project - reflects on these meditations. Albert prioritises direct interaction between the designer and an evolutionary algorithm to train the system to execute solutions that are customised to designers' preferences (Aksöz, 2019). It provides a graphical user interface (GUI) with which the designer can easily compare different design variations by reviewing visual representations and their relative performance. Through direct interaction, the designer can compose sets of preferred solutions and manipulate computer-generated solutions. By merging interactive evolution with the manual intervention of the designer, an environment of exploration and negotiation is established that smoothly integrates with the early design phase. This avoids long calculation periods and the potential isolation of the designer from the exploration process. Thanks to rapid evaluation, designers can make decisions regarding which parameters and objectives to select for the preferred design goals, and subsequently update or restructure the parametric model.

Although this method can be readily integrated into creative exploration, there are limitations to a parametric approach to design. To successfully implement an exploratory method, one still has to define the design parametrically. Establishing a model from very early on can be tically. Establishing a model from very early on domain of exploration remains within the boundaries of selected variables. Therefore, in the very ealy oxplorations, designers still prefer to work with 3 . modelling , designers of prefor to work with 3D modellig as a merhod more fexiblity and supports ambiguity. How can we integre that the don the design problem? Here, another type of interaction can be discussed, whereby both designer and computer can influence design outcome on different layers of complexity, emphasising their individual qualities. The designer has the ability to control the creative process from a holistic point of view. The computer, on the other hand, can process complex calculations in a very short period of time. With the integration of machine learning calculations can be learnt, based on experience, without being explicitly programmed. ANNs are able to quickly execute results in parallel, while the computer can conduct adjustments, changes or improveme in detail, informed by previous experiences.

The different levels of design can be distinguished as 'global' and 'local'. While the designer is intuitively exploring the global design outcome, the computer can focus on the local details and execute solutions regarding performative goals. To summarise this process, we can go back to the parametrisation of the project. In this context, the designer can control the overall curvature, the overall geometry and the triangulation strategy of the façade, while the computer can operate in detail, fixing the element sizes and improving structural performance. Instead of using a constraint-based solver that can only adjust the geometry in line with given constraints, the ANNs can be trained to execute solutions regarding multiple criteria, not only based on geometry. The problem description becomes so generic that the same ANN can be used on different projects, executing completely different solutions. Consequently, instead of a problem-specific approach, a domainspecific approach can be developed that can prove more sustainable for the overall design workflow.

\section{Conclusions}

The integration of machine learning and artificial intelligence-based processes encourages a shift in computational design. Designers are confronted with a new approach that challenges traditional methods of thinking by motivating them to focus on design performance early on. This approach can be beneficial, integrating harvested data with creative exploration. Awareness of design performance can thus be rais. which helps reduce long optimisation cycles in later stages. These systems should be approan in latically however and used as tools to negotiate between the quantitative and qualitative goals. They should remain an extension and an amplification of creative exploration that supports the ans The underlying auestion, then, becomes: 'How can we The underlying auestion, then, becomes. How can we establish a fusion between he ambiguous nature of processes that smoothly migrate into this phase?
Thesis

Aksöz,Z., 2019, Reflections on Multiple Criteria Optimization in Early Design Phase Learning for Human Machine Machine University of Applied Arts Vienna. Supervisor: Prof Klaus Bollinger. Industria partner: Dr Julian Lienhard, str.ucture,
Lightweight Design made in Stuttgart,

\section{Bibliography}

Fischer, G., McCall, R. and Merch, A., 1991 Making Argumentation Serve Design', in
Human-Computer Interaction, Vol. 6, No. 3-4, p. $393-419$. do:

1080/07370024.1991.9667173

Gärtner, B. and Hoffmann, M., 2013, HS 2012: Voronoi Diagrams' ch/ew/Lehre/CG13/lecture/cg-2012 pdf (accessed 7 February 2020).

Harding, J., and Shepherd, P., 2014, (1) https://doi.org/10.1016/j.destud.2016.09.005 (accessed 7 February 2020).

Mothersill, P. and Bove, V.M., 2017, 'Humans Machines and the Design Process: Exploring the Role of Computation in the Early Phases of Creation', in The Design
Journal, Vol. 20, Sup.1, p.S3899-S3913. do 10.1080/14606925.2017.1352892

Piker, D., 2013, 'Kangaroo: Form Finding with Design, Vol. 83, p.136-137. doi:10.10021 ad.1569

Rittel, H., 1972, "On the Planning Crisis: Systems Analysis of the "First and Second p.390-396.

Nerlinger, R., 2013, 'Multi Objective Design Interface, Technical University of
doi: 10.13140/RG.2.1.3401.0324 


\section{Computational Extensibility and Mass Participation in Design}

Paul Poinet

The Bartlett School of Architecture, UCL

Al Fisher

BuroHappold Engineerin

\section{Introduction}

In order to manage the design-to-fabrication process of large-scale and complex architectural projects, Architecture, Engineering and Construction (AEC) companies have been independently optimising custom in-house processes, as well as the delivery of data to external partners (Deutsch, 2017). These self-organised ways of improving workflows can take different shapes, depending on the company's specific activities. For example, companies that conduct construction-related tasks and deal with large and complex datasets at late stages can be seen to primarily focus on developing tailored scripts based on existing software platforms (e.g. Design-to-Production, Front Inc., Woods Bagot) that curate the generated data from early to late stages until the completion of the building. Meanwhile many consultants and AEC software developers have built custom interoperability tools to bridge the communication rap between different software phatforms and further, a to senericise these to to gencicise (e.g. Proving Ground's plug-ins). Even though all these custom processes, tools and sofware are valuable in solving immediate and critical issues related to the project, working in isotion they are naturally not froject, working in isolation hey are naturally not focused on - and therefore cannot address the wider challenges of - data interchange, collaboration and

Thefficiency across the complete supply chain. The objective of the work described in this chapter is use design and code experimentation to solve local problems related to development, deployment and reuse within the practices mentioned above; and, importantly,

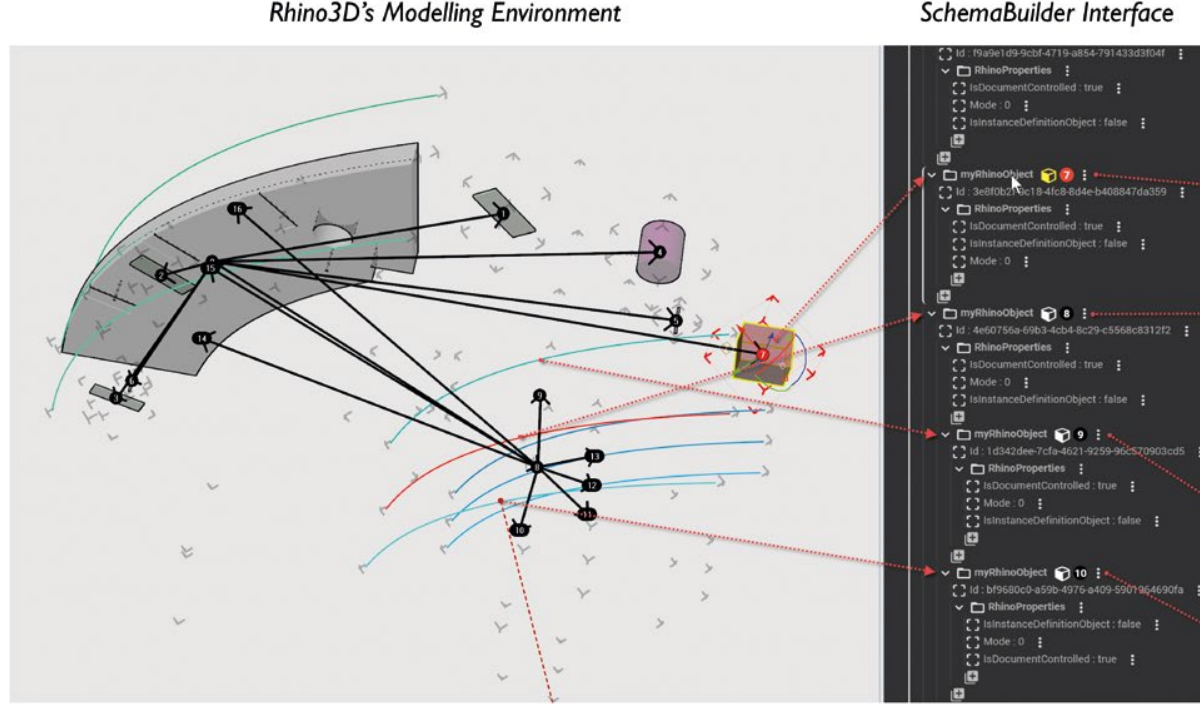

stage. For the AEC industry this means open-source computational designer, engineer and architect in an accessible computational design and coding ecosystem.

Open-Source Frameworks: Enablers of Mass Participation and Computational Extensibility in Design Practices

The challenges for achieving the widespread adoption of computational methodologies are threefold:

Construction of design project and system architectir

. Skiling of core competen

3. A technology plours:

$m$ that facilitates and reinforces distributed development, co-creation, system

redundancy and flexibility, while nurturing innovation through prototyping.

These challenges necessitate the development of new coding and novel collaboration paradigms, the details of Which are compatible with mass-distributed co-creative processes. These are demonstrated in the three case studies here, which range from modelling experiments tore robust prototypical applications. platforms for combining the efforts of the programmer, been used - separately or orchestrated together - in order to demonstrate the potential of computational extensibility (enabling better transparency and interaction between the different design actors at every stage) and mass participation in design practice: Buildings and Habitats object Model (BHoM) and Speckle:

- BHoM has been designed as a hybrid model for code architecture, integrating a number of concepts across existing languages and platforms. The BHoM has data structure and manipulation strategy that is directly comptible with both visual flow-based programming (such as in Grasshopper3D and/or Dynamo) and text-based imperative code. Practicaly, Drot

define design objects that can be converted to and from various software platforms. In this neutral environment proprietary software functionality can be extended by adding and calling generic methods through system reflection.

Speckle is an extensible and scalable design and AEC data communication protocol and platform. It offers a neutral schema for the specification and creation of basic geometry types, as well as a federated server architecture to send and datasets defined by the user within differen oftware platforms. receive streams that collect various geometric
1. The SchemaBuilder user interface
enables the end user to create hierarchic elationships between Rhino3D objects user-defined schema containing different objects and sub-object 
The three case studies described here gradually tackle different scales, from local object level to macro project level. As the scale increases, more design participants become involved in the process, resulting in complex design workflows and behaviours. These prototypical experiments respectively highlight three main points:

1. Extensibility and flexibility of the object's schema; 2. Cross-practice collaboration and interoperability; 3. Project and process mapping at scale.

\section{Flexible and Extensible object Schemas}

Design object flexibility and extensibility are crucia aspects to be enabled during the conception of large-scale digital workflows. This means that any data schema needs to be flexible enough to adapt its representation to any unknown future change or requirement throughout the design process, without losing its core abstract definition necessary for robust interoperability and sharing across all required software platforms. In order to explore designer control of such object customisation, a prototypical application entitled SchemaBuilder has been developed. SchemaBuilder's main goal is to allow the user to build custom-nested hierarchies of geometrical objects. The user is able to select geometries directly from the viewport in Rhino3D and aggregate them within a directory tree structure ${ }^{4}$ from which custom properties and 'parent-child" relationships can be defined. As the hierarchy is bein built, a corresponding graph highlighting the current dependencies can be previewed within the viewport. Once the user is satisfied with the object's schem the latter can be shared on an online server using the Speckle plug-in for Rhino3D.

SchemaBuilder focuses exclusively on the object's properties and metadata Other strategies have look at similar extensibility of an object's methods and behaviours. The BHOM framemork mentes the inject and exposition of an object's methods as well as its and exposithor properties. Exposed in avisual programming User tree of objects and call a spectic object's methods.
Methods that can be injected into the object from multiple authors, across separate modular code projects, open the door to mass customisation for disparate purposes.

In both the SchemaBuilder interface and the BHoM code framework, flexibility and extensibility have been tackled on the local, small-scale level of the object. The next step is to demonstrate the benefits of adaptive object schemas ${ }^{5}$ through a cross-collaboration case study that involves two different collaborators working on a common object. This context is illustrated by the next modelling experiment, which is a speculative cross-practice collaboration between BuroHappold Engineering (an engineering practice based in London) and Design-to-Production (a consultancy practice based in Zurich).

Cross-Practice Collaboration

To demonstrate the potential of the previous experiment within a collaborative scenario, the following speculative experiment attempts to integrate the communication and collaboration processes between Design-toProduction and BuroHappold Engineering through schema-based workflows, using a specific example of a timber assembly modelled by the former. In this experiment, a common schema has been shared between the two practices, which are working toward a common project goal but with different design objectives: while Design-to-Production is generating full geometrical descriptions of each architectural component, BuroHappold Engineering is mainly focusing on obtaining precise structural analysis results. To perform the latter, BuroHappold Engineering uses the $\mathrm{BH}$ OM platform, allowing seamless data transfer from the Grasshopper canvas - considered herser main Ul interface of the BHoM - to Autodesk's Robot main Ul inale Analysis 6 software Structural Analysis sof ware package. The retained common schen wh wiscumsed by both partners, to generate their respective dar a a planes inforing on the directionality of the beren planes inf and tructura anasis Such areementon common-object modelenabs abi-directional

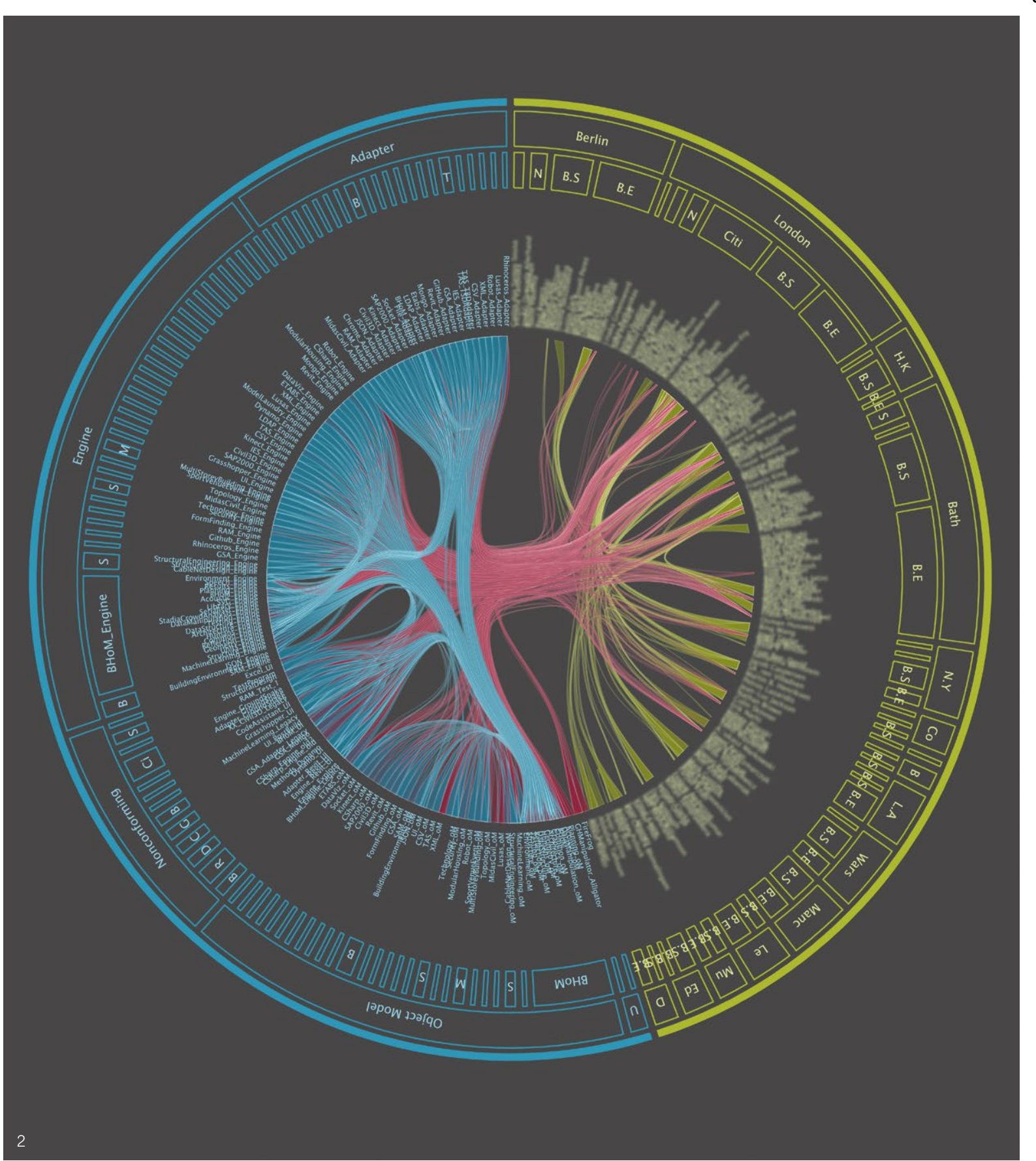



between the respective working environments of the two companies.

This scenario shows how a single data-rich object is able to adapt to two different paradigms, from engineerin to fabrication concerns. The third experiment of this series aims to scale this speculative design workflow further by shifting the focus from the object to the project scale.

\section{From the Object Level to}

\section{the Project and Process Scales}

Visualising and mapping a large number of object instances from an entire project can be quite challenging, especially for complex modelling consultancies like Design-to-Production with requirements to describe at full fabrication resolution all necessary parts of a building. In order to ease the design process, therefore, an interface entitled LayerStalker has been prototyped, presenting an alternative means of rendering object dependencies and exploring complex datasets and geometrical information of large-scale projects. A sunburst diagram is used to visualise the hierarchical structure of the layer table from Rhino3D. 'Child' layers are represented as offsets of their respective 'parent' layer, situated more toward the centre of the diagram. Each level is subdivided by 'sibling' layers.

Through the LayerStalker interface, the user is able to perform unstructured queries using specific tags ('detailed volume', 'dowel', 'drill', 'axis', 'connector', etc.) to search the local database, to display all objects with layer names that contain the exact same tag within th application's viewport Although acting here on a local database only, the LaverStalker interface could be implemented within either the BHoM or Speckle frameworks to operate web-based queries, enabling framear design communication and mass participation in design practice at a larger scale.

Conclusion: Toward Mass Participation

The three examples outlined in this chapter focused on the local adaptive object schema, its transfer between different modelling environments and its instantiation at the project scale. Merging these three concepts enables cross-practice collaboration at scale, whereby multiple parties can seek to exchange complex data schem and (sub-)models in a project.

This principle was explored during the 2018 Innochain Simulation for Architecture + Urban Design (SimAUD) workshop taught by Dimitrie Stefanescu and Paul Poinet. The workshop aimed to introduce the Speckle opensource framework and focused on open, collaborative design and modelling workflows. As part of the exercise, a predefined modelling workflow consisting of generating a complex network of freeform timber beam elements was segregated into six distinct modelling pipelines that was shared amongst the six workshop participants through Grasshopper files. Those files were linked through Speckle senders and receivers. The geometrical data was incrementally streamed to a Speckle viewer at each data transaction. The streams could also be aggregated and displayed within a common viewer accessible to all participants. Each time that pipelines were connected through the object streams, the overall workflow's map could be visualised in real-time through a global, higher-level graph displaying the input-output connections between the different pipelines. It is this 'metagraph' that is the key concept here, keeping track of both the object and the process and, crucially, human dependencies that exist, adapt and change throughout the evolution of a project.

As previously mentioned and observed in all the above modelling experiments, the human factor cannot be neglected in favour of a purely automated technological paradigm, and must be considered throughout all aspects of the design process. In order to enable mass participation in design at scale, co-creation and co-authorship behaviours need to co-creation and co-authorship behaviours need to and team competencies.

\section{Competencies and Behaviours}

To enable this continuum of participation from entrylevel computational skills to advanced development, BuroHappold Engineering has developed a series of Computational Competencies, ranging from Level 1 :
'Appreciation of Visual Programming' to Level 4: 'Expert Knowledge of Text-based Programming'. The former focuses on Grasshopper and Dynamo and the abstract concept of flow-based programming, while the latter, relates to advanced, well-structured modular C\# code, co-created through collaborative behaviours facilitate through platforms like GitHub. The important intermediate levels 2 and 3 ensure the co-creation, sharing and reuse of VP scripts, and a smooth transition to basic coding.

A continuous range of computational skillsets enables mass participation in computational design, both in terms of usage and authorship. The distributed co-creation of the BHoM code base brings the benefits of the creators' diversity, resilience in development and greater relevance for adoption.

\section{Project Architecture}

With both an increased talent pool and a technology platform crafted for deployment at scale, the key to enabling effective utilisation is to ensure development is in context - that it is distributed on projects and, in the case of large multidisciplinary firms, across both project teams and disciplines. This requires the formulation of project architecture and a project team and leadership that are equipped to facilitate development and ensure effective and appropriate use.

Instilling cultural change across all three of the above pillars enables a foundational change in an organisation's computational capabilities. In a similar way that DevOps (Development and Operations) has been seen as unifying software development and operations, the authors see computational engineering as a conscious unifying of continuous computational development and utilisation of code on projects, driven by project needs and performance outcomes.

The natural implication of a distributed development approach, such as that proposed by this escay is the exploitation of network effects in intelligence and - through improved communication - participation -ccess and the means to utilise this corticis collective know how. The seres of modelling experments the progress made toward establishing such an approch.
Bibliography

Deutsch, R., 2017, Convergence: The Redesign of Design (AD Smart). Modeling to Building' in Marble, S. (ed.) Digital Workflows in Architecture:
Design-Assembly-Industry $110-131$ Design-Assembly-Industry, p.110-131,
Basel, Birkhäuser.

Speckle: Open Digital Infrastructure for Designing, Making and Operating the Built
Environment, 2018. https://speckleworks (accessed 7 February 2020).

The Buildings and Habitats Object Model: shom.xyz (accessed 7 February 2020).

van der Heijden, R., Levelle E. and Riese, M Generation for Design and Construction in Computational Ecologies: Design in the Anthropocene: Proceedings of the 35th
Annual Conference of the Association for (ACADIA), Cincinnati, Ohio. http:///papers. cumincad.org/data/works/att/acadia15_417.

\section{Notes}

1. In computer science, a visual the user to create programmes by manipulating elements graphically

\section{rather than by specifying them textuall}

2. Imperative programming is a programmin
paradigm that uses textual statements to paradigm that uses textual st
modify a programme's state.

3. See p.62-67 and p.80-81 of this volume. 4. In computer science, a directory structure
is the way an operating system's fis is the way an operating system's file system
and its files are displayed to the end user.

5. While object schemas are usually static and specified by an object-oriented vendor, the authors argue here for dynamic object schemas that can be changed and 6. www.autodesk.co.uk/products/
robot-structural-analysis/overview 


\section{Alternative Means of Digital Design Communication}

Dimitrie Stefanescu

The Bartlett School of Architecture, UCL

\section{Introduction}

Design, in general, is charged with solving ill-defined, or 'wicked', problems, the understanding of which is concomitant with the act of their resolution. In the words of Rittel and Webber, 'the information needed to understand the problem depends on one's idea of solving it' (Rittel and Webber, 1973; Crowley and Head, 2017; Rith and Dubberly, 2007). As such, the act of design has no definitive stopping rule, and nor can its output be judged by a binary evaluation of good vs. bad. Most importantly, the stakeholders involved in the design process do not necessarily have a shared set of values. Essentially, design can be seen as an iterative act that aims to reduce uncertainty at an antorical act (Hanna 2014) by simultaneously searching for the appropriate problem-representation and resolution

The main goal of this research project as set out in the original Innochain call, is to analyse how complex, digital-based design can be communicted and coll internally, within a can be comm team, and externally, with the various stakeholders involved in the design process. The various stakeholiders the iterature establishes that communication pand dlalogue as, through these means, shared understanding can be as, through these means, shared understanding can be constructed among the actors involved in the (design) process (Dawes et al., 2009, Lawson, 2005, Roberts, 2000, Conkin, 2005, Walz et al., 1993, Bechky, 2003). In otherwords, shared understanding can be construed as a set of matching ontological representations of meaning that gradually emerge through a process of conceptual displacement (Koestler, 2014, Schön, 2011 1991). Communication and dialogue are increasingly reliant on digital means, yet they cannot be fully analysed hrough the lens of a purely technical model. On this premise, communication is understood as a transactional manifestation (digital) and also an intrinsic psycho-social component. The former approach corresponds to the Shannon-Weaver model of communication (Shanno and Weaver, 1963, 1948), while the latter draws from inferential models developed under the philosophy of the natural language communication field (Grice, 1991; Sperber and Wilson, 1995; Wilson and Sperber, 2008). Consequently, this project aims to investigate critical aspects of design communication, whereby communication is understood as having both technic and social dimensions that reinforce each other (Garfinke and Rawls, 2006). Following that, at the beginning of the design process, one is bounded by an incomplete definition of the given problem and by the fact that the relationships between actors need to first emerge the subsequent shared ontologies can be defined. With this in mind three main research directions were selected: (1) data the mentation and (2) data clasification juxtapose different approaches to ontological models juxtapose difent of design objects, and, inally, (3) data transaction looks they change the nature of design communication.

\section{Methodology}

The comparative investigation in the three research directions outlined above lacked a feasible alternative to existing digital design communication software. Consequently, this project required a separate software platform to be developed that would offer a valid

comparison base. These efforts resulted in the connected design platform named Speckle, which served as base reser instrument throughout this project.
First, the Speckle platform can be described as being schema-agnostic: it does not have a standardised ontology per se, but rather a small set of user-defined, composable object models that can be swapped in and out, or used in tandem. Second, Speckle is objectcentric, as opposed to file-centric: instead of saving data in monolithic blocks, it stores each object individually (and immutably) and allows for overlapping groupings thereof. Third, Speckle is embodying data: as opposed to existing approaches, where files are just 'shared' and there is no overview of who is consuming the information, nor to what effect, Speckle traces the communicative network and informs end-users of their transactions and the implications of these. Thanks to these characteristics, Speckle enabled theoretical technical and applied analysis of the forementioned research questions. The collaborative network within which this research project was undertaken, Innochain consisted of both acamic and industrial partners. It served as the seed for a living aboratoy that grew throughout the project to folve many otherindustry participants As such, this setting served both as the basis for the technical devis from which the auantitative and auditative as the pool to investigate the research directions outlined above to investigate ther.

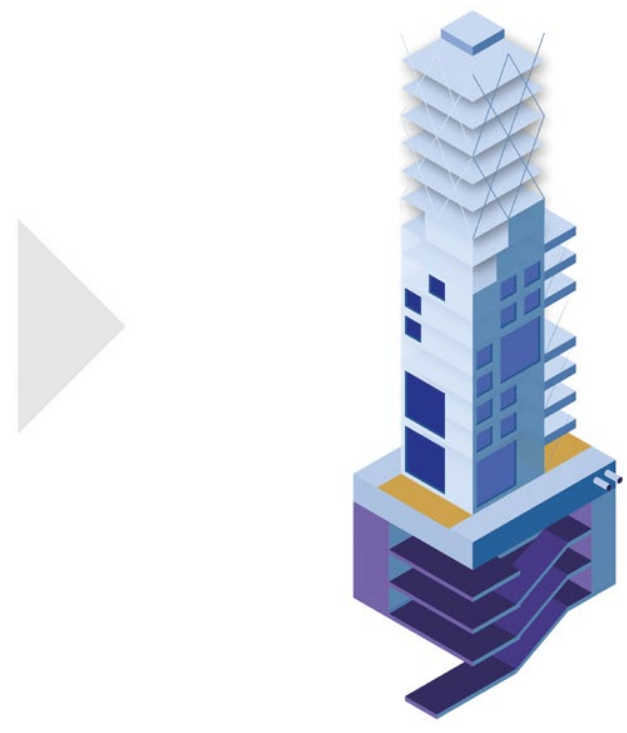

Research Findings

Regarding the first research direction, (1) data representation, it was found that a low-level composable schema provides opportunities for dialogue between stakeholders on the topic of how design objects are defined - essentially, enabling ontological revision at a representational level. Furthermore, existing higherlevel object models can be natively supported, including the industry-standard Industry Foundation Classes (IFC), without loss of fidelity and without enforcing an overly strict high-level standard. Nevertheless, one important limitation was the asymmetrical codification and de-codification of objects, which can lead to information loss. Specifically:

A lower-level, composable object model allows undergo a productive process of ontological (representational) revision.

Existing higher-level object models from the industry, such as the BuroHappold Object Model (BHoM) or IFC, can be natively supported, thus Allowing for 'backwards-compatibility'.

Multiple, self-contained object-models can be programmatically supported in a simultaneous and consistent manner in a digitally-enabled esign communication process, thus invalidating (n) 


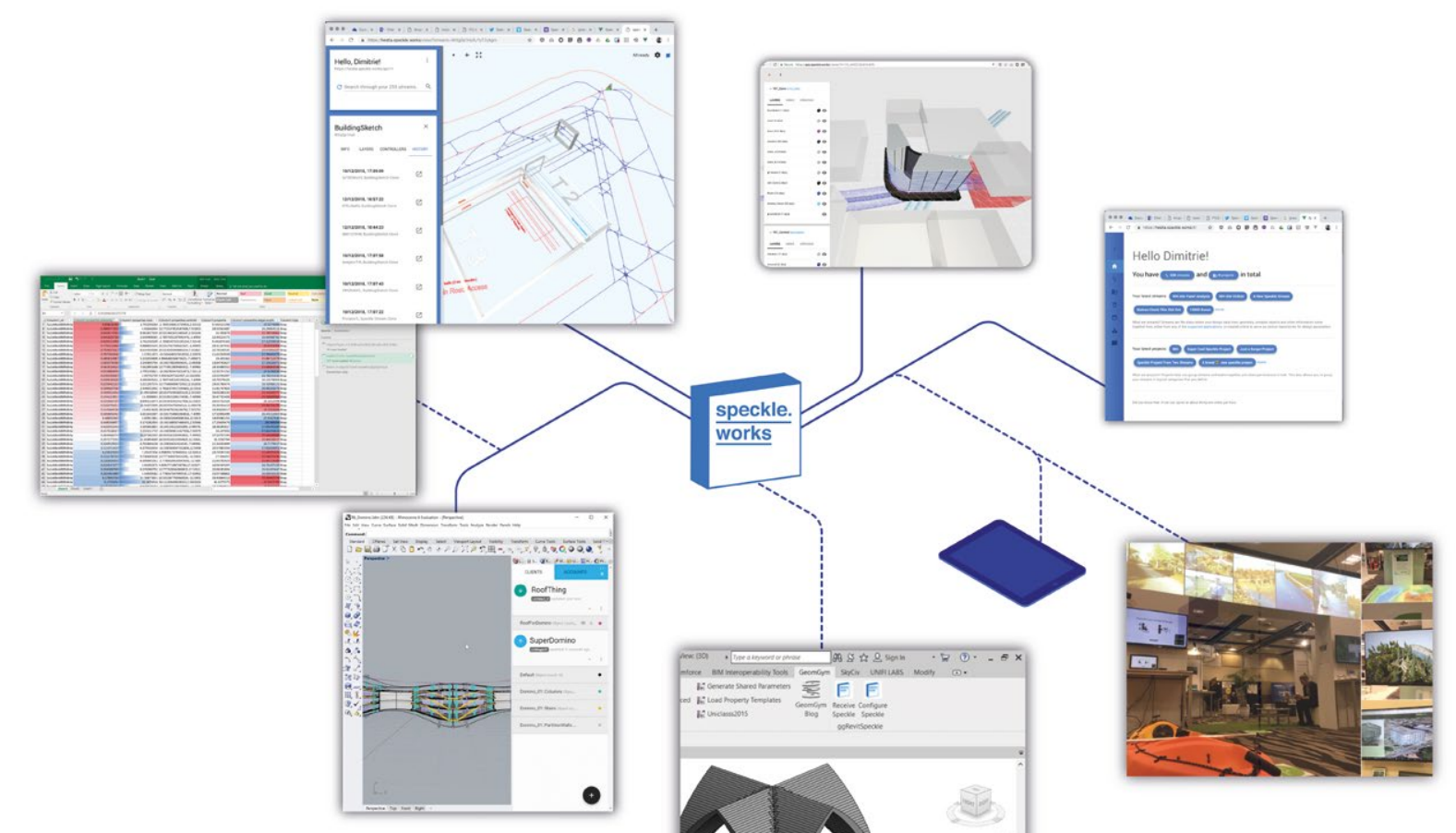

In (2) data classification, we put forward a curatoria object-centric approach to data storage and classification that mitigates information loss. This was achieved through an approach to object identity that was

directly linked with the object's properties, as opposed

to a randomly-senerated one of the type currently

employed in most persistence layers. Furthermore,

we showed that unlike file-centric collaboration

methodologies, an object-centric approach imposes

ontological revision at the content level by requiring

actors to negotiate what information they share and why.

In turn, this leads to increased relevance of data and a

reduction in overall communication noise:inste reduction in overolion in buk', any design data that is sharing information 'in buk', any design data that is The findings of this second resipient and a direct use The findings of mis second

- The communicative productivity of an object-based curatorial approach to design data classification has been validated both through qualitative means, by assessing several case studies observed 'in the wild', and through empirical observations based on the and through empirical observations based on the monitored usage of Speckle: on average, dese models were broken down into 2.47 separate
sub-classifications. Furthermore, when taking into sub-classifications. Furthermore, when taking into account the sources coming into a given model, the
average count of both sources and receivers per model was 2.78 , highlighting a dynamic process of fragmentation and re-assembly.

- An object-based approach to data persistence is potentially twice as efficient as a file-based one in enabling multiple overlapping classifications of design information. Furthermore, the actual cost of creating new classifications from existing ones is virtually negligible (< $0.1 \%$ of the original model size), which facilitates the emergence and evolution of efficient 'informal' communication exchanges.

Finally, in strand (3) data transaction, by evaluating key characteristics of the informational flows (their frequency, the potential for meta-information transmission, their relevance our meta-information contexts), contexts), We showed hat smaller (and thus faster) data transactions increase the velocity of the overal communication process. This determines the rate at which the representational and cortent-level revisions proced, and thus represents acilical aspect of dgt design communication in terms of enabling the major findings were:

- The transaction size can be decoupled from the size of the model, thus enabling much smaller and speedier, change-dependent digital design data exchanges. From a theoretical point of view,
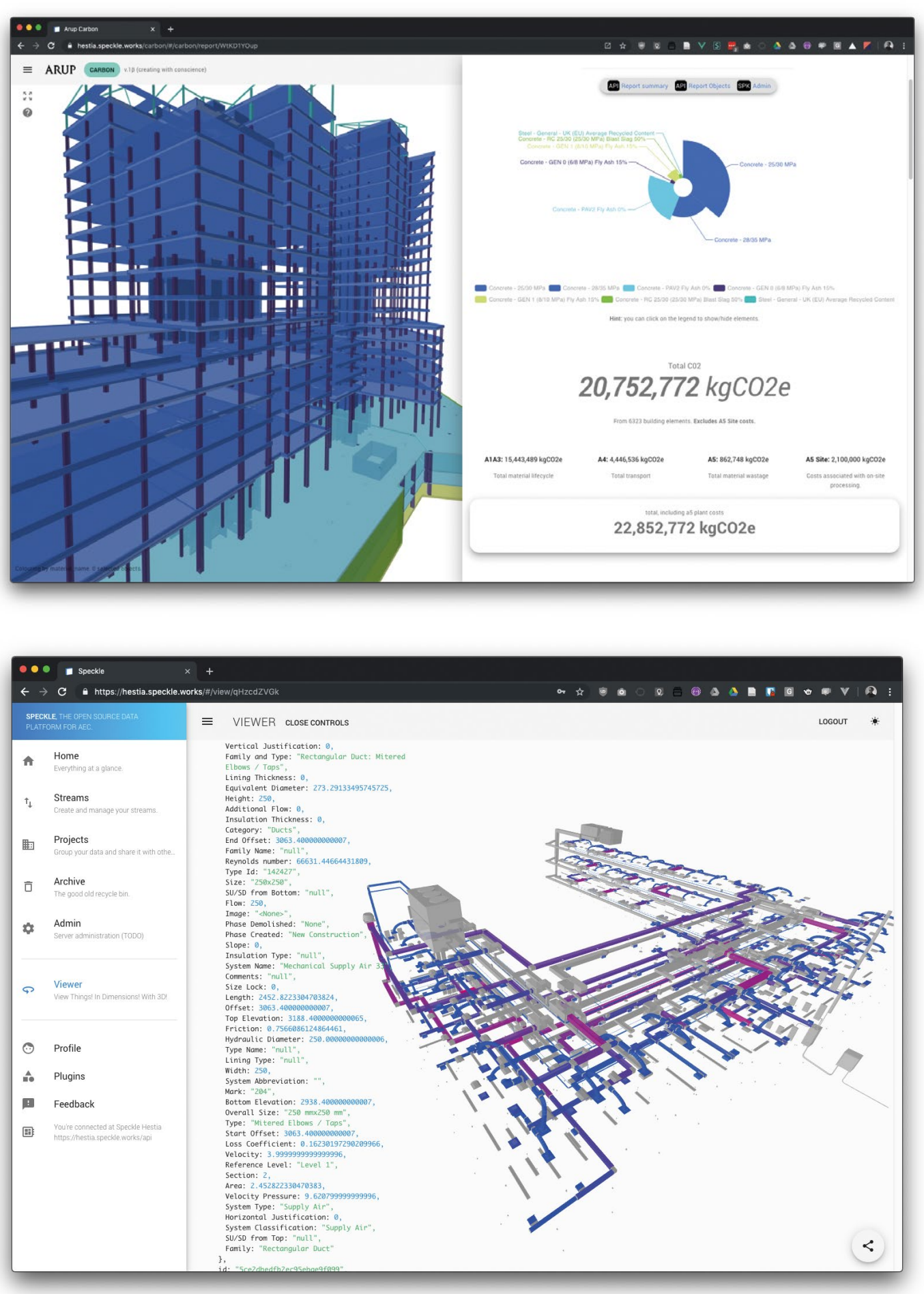

2. The Speckle ecosystem connects enables customised digital worktlows to be assembled.
3. Arup Carbon, a proprietary embedded developed on top of Speckle to provide real-time sustainability insights on design
4. Mechanical engineering model and its associated data
Speckle viewer. 


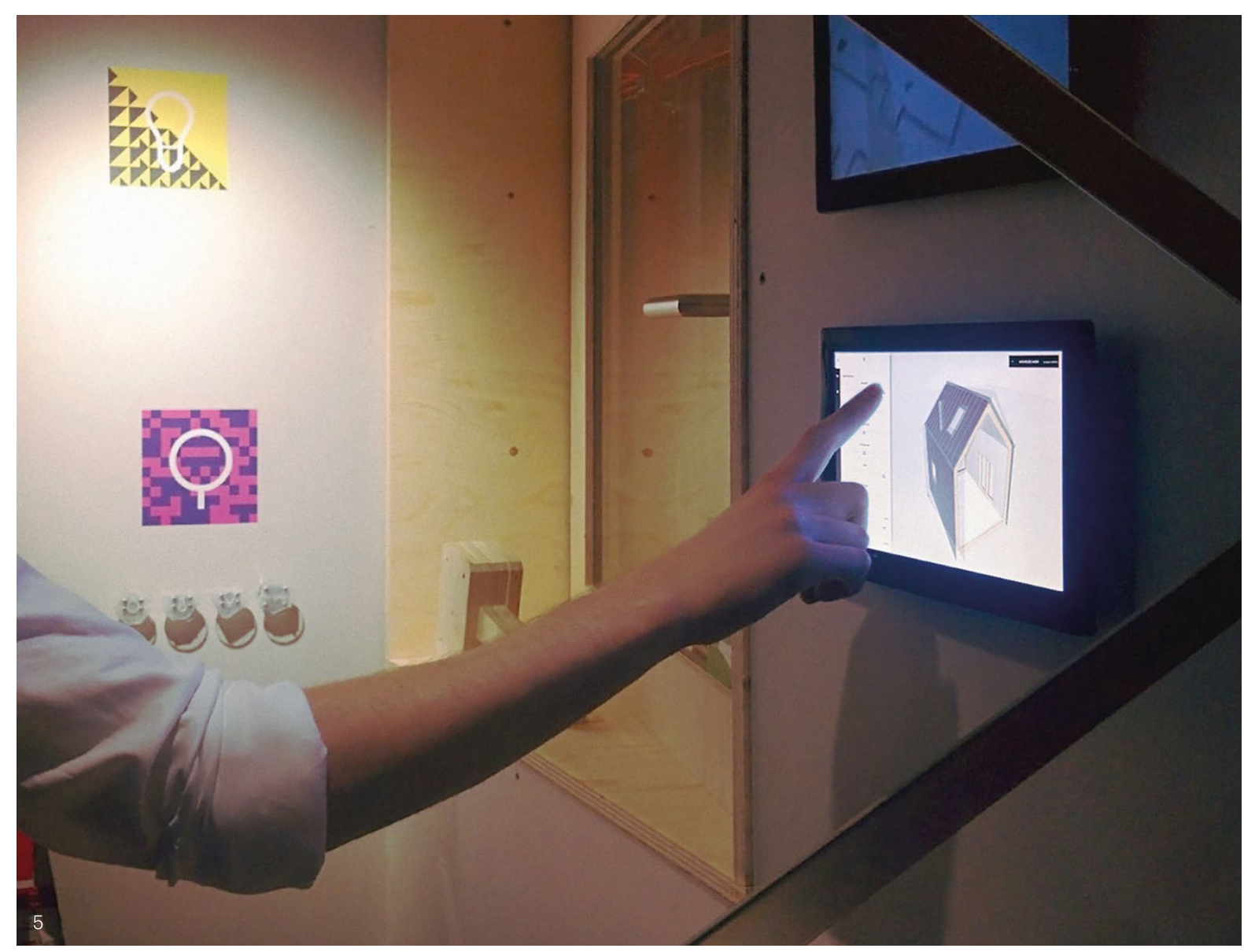

this allows for the reassertion of nextness (adjacency) in a digital design workflow. Empirical observations showed that transactions can be as much as five tim faster (by virtue of being five times leaner in size).

- Informing users of who is dependent on their data (as well as when data on which they are depending, coming from a different person, has become stale) establishes a measure of productive sequentiality. which is sufficient for individual tasks. Nevertheless, weidentified a need for stakeholders in coordination which is sufficient for individuatasks. Neverheless, roles to provideapr

\section{Impact and Perspectives}

In terms of ongoing academic research, the most recent development is the 'AEC Delta Mobility' grant, funded by Innovate UK in late 2018. Within a consortium consisting of BuroHappold (former Innochain industrial partner), 3D Repo, Rhomberg Sersa Rail UK, Speckle, and The Barlett School of Construction and Project Management at $\mathrm{UCL}$, the scope of this project is to normalise and specify, using Speckle, an industry
Digital communication constitutes a key infrastructura base on which the design process now operates. Withi this research project, communication was contextualis as a transactional phenomenon, with both technical and social manifestations that einforce each other. Accordingly, the contribution made by the project can be summarised as an integrated technical and sociological rethink of communication in the digital design process that challenges the existing status quo of the AEC industry.

By marrying contemporary technical affordances with a user- and industry-centred analysis, this project demonstrates that existing assumptions around the need for centralised high-level standards and workflows discourage meaningful dialogue from happening and exclude vital stakeholders from the design process.
By contrast, a flexible digital communication Brovides inferential context for dialogue to take place among design stakeholders, allows for emergence and evolution in the way formation is defined and structured, enabling the creation of shared values and meaning. Broadly speaking, the low impact of emerging technologies on the overall productivity and efficiency of the AEC industries (Barbosa et al., 2017; Charef et al., 2019; Dainty et al., 2017; Hong et al., 2019) can be attributed to widespread confusion regarding its communicative processes, and their subsequent distortion through inadequate technological implementations. Nevertheless, this research project concludes that digital technologies can embrace the diversity and richness of the design industry, and, moreover, open an accessible and ethica pathway toward a digitally-integrated built environment.
Bibliography

Barbosa, F., Woetzel, J., Mischke, J., M., Bertram, N. and Brown, S., 2017, Reinventing Construction: A Route to Higher Productivity, Washington DC,

Bechky, B.A., 2003. ‘Sharing Meaning Thess Occupational Communities: on a Production Floor' in Organizatio Science, Vol. 14, p.312-330.

Charef, R., Emmitt, S., Alaka, H. and Modelling Adoption in the European Unio An Overview' in Journal of Building Conklin, J., 2005. Dialogue Mapping:
Building Shared Understanding of Wicke Problems. Chichester: Wiley.

Crowley, K. and Head, B.W., 2017. Problems": Revisiting Rittel and Webber" . Dawes, S.S., Cresswell, A.M. and Pardo, T.A.
2009, "From "Need to Know" to "Need to Share": Tangled Problems, Information Sector Knowledge Networks' in Public Administration Review, Vol. 69, No. 2 concluces that ithas been shown that the approach of engagement and support the exchange between expert and laymen in urban design?

With regard to adoption within industry, a number of international AEC companies have incorporated Speckle at the core of their digital transformation efforts. Among many others, the most prominent firms are HOK, SOM,
Dainty, A., Leiringer, R., Fernie, S. and Harty, C., 2017, 'BIM and the Small Construction Research \& Information Research \&
p.696-709.

Dainty, A., Moore, D. and Murray, M., 2006 Routledge.

Garfinkel, H. and Rawls, A., 2006, Toward Sociological The
CO, Routledge.

Grice, H.P., 1991, Studies in the Way of Word
Press.

Hanna, S., 2014. 'Ontological Uncertainty nd Design: Requirements for Creative Creativity Workshop 2014, London.

Hong, Y., Hammad, A.W.A., Sepasgozar, S, Model for Small and Medium Construction Organisations in Australia' in Engineering, Construction and Architectural https://doi.org/10.1108/ECAM-04-20170064 (accessed 10 February 2020).

Lawson, B., 2005, How Designers Think: The Design Process Demystified,
4th edition, Amsterdam, Routledge. Koestler, A., 2014, The Act of Creation,
Rith, C. and Dubberly, H., 2007, 'Why Horst W.J. Rittel Matters,
No. 1, $0.72-91$. Rittel, H.W.J., Webber, M.M., 1973, 'Dilemmas
in a General Theory of Planning' in Policy

Roberts, N., 2000, 'Wicked Problems and Network Approaches to Resolution', in

Schön, D.A., 1991, The Reflective in Action, Farnham, Routledge.

Schön, D.A., 2011, Displacement of D. Mathematical Theory of Communication University of Illinois Press.

Sperber, D., and Wilson, D., 1995, Relevance: Communication and Cognition, 2edition Cambridge, MA, Wiley-Blackwell.

Walz, D.B., Elam, J.J. and Curtis, B., 1993, Acquisition, Sharing, and Integration' in Communications of the

Wilson, D. and Sperber, D., 2008. 'Relevance Theory', in Horn, L.R. and Ward, G. (eds.), MA, Wiley-Blackwell, p.606-632. 


\section{Negotiating Structured Building Information Data}

Nathan Miller and David Stasiuk Proving Ground

The Emergence of Machine Learning

In recent years, applied machine learning techniques and advanced algorithms for data analysis have become increasingly ubiquitous across a wide array of professiona and academic practices, ranging from the natural sciences to business and finance. The successful application of these algorithms hinges on their access to large volumes of data describing respective targets of inquiry. In machine learning, input data is organised into features, which may be produced using a variety of independent mechanisms.

The heterogeneity of input data carries both positive potential and risk: while diverse input data may vield better results, it is generally a non-trivial effort to effectively capture, reconcile and integrate multiple discrete data sources. For example, decision support resulting from a sales analysis that relies on the observation of business transactional data may be significantly enhanced or further informed by customer attitudinal profile data; or scientific date describing natural phenomena collected through sensors or other tech with data generated through the application of computational simulations. Yet, the synthesis of such distinct datasets into meaningul-feature collections may require manual processing that relies not only on the ability and intuition of the user implem net only alsorithm butaso the ounlity and 'clecilness' datif $T$ ine value of these analycal techniques and decision support ools is thus tied to the unniques and which they rely on being correct, suitably voluminous and well structured.
Challenges for the Application of Machine Learning in

Architectural Design

Architects and engineers have an established history of using machine learning algorithms to enhance manufacturing processes (Nicholas, 2017; Brugnaro, 2017; De Leon, 2012), search intractably large design spaces (Stasiuk, 2014; Derix and Thum, 2005), label spatial typologies (Peng, 2017) or even generate building and floor plans (Chaillou, 2019). These techniques have, however, more rarely been applied as decision support tools for associating early-phase design and discovery processes with specified outcome quality especially related to more subjective performance measures. Th related to more subjective performance measures. The relative absence of the use of predictive modelling for performances stems from multiple challenges. The first of these relates to problem definition: difficulty in effectively articulating subjective design problems has been considered extensively becign problems has be consider introduction of adesign sol (Rin may, in fact, introduce ew, un to challen problems (Ritel, leads to challenges in quantifying performance for those that ive to these stated problems, especially for those that appear outwardly subjective, such as a user's experience of the space. Furthermore, in practice the types of well-structure dain or have access to the types of well-structured data needed for training machine learning algorthms to become effective mechanisms for outcomes prediction. As a result, the most abundant examples of applied machine learning in the building sciences tend to focus on the physical characteristics of an assembly, such as its structural or

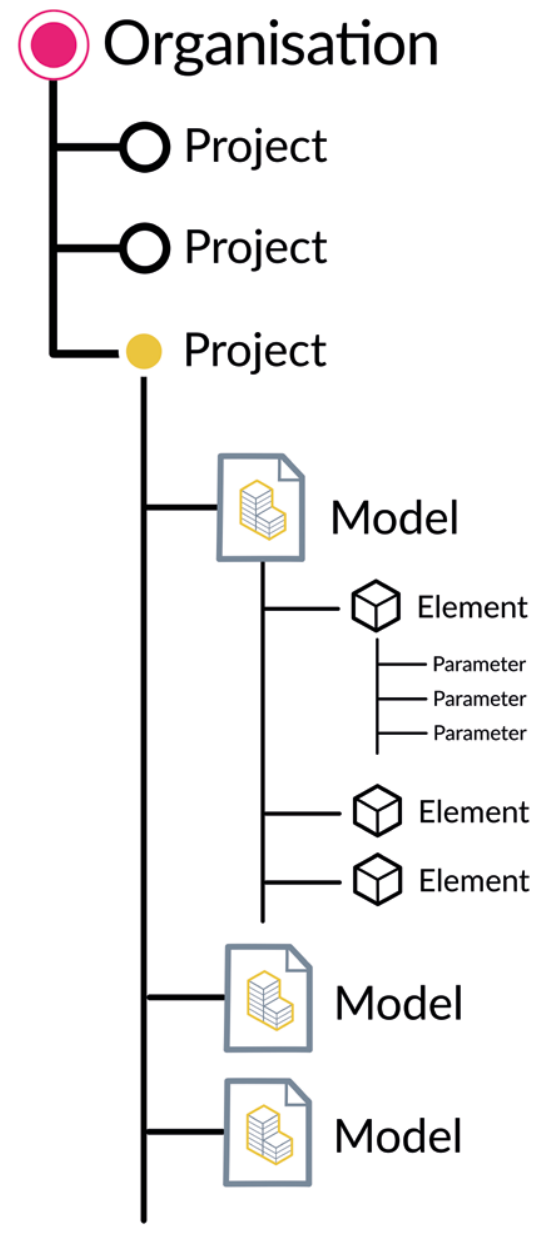

The rare examples that engage in predictive modelling for architectural usage help illuminate reasons why. One such approach has been described by Daniel Davis, where his company WeWork leveraged historical data to optimise its conference-room design methodologies for end-user satisfaction (Davis, 2016). Here, the vertical structure of WeWork - a company that designs, own and operates its own buildings - facilitates the acquisition and curation of datasets created at differen stages along the supply chain. Their relative success relies on the extensive data access afforded by the multi-faceted roles their company plays in the building lifecycle: this underscores the challenges in realising closed-loop predictive analyses faced by more traditional design practices that generally only have access to design-level model data.

Methods for Exploiting Model Resources

Contemporary architectural projects of even the most modest complexity are ubiquitously realised using digit modelling techniques for design authoring and for the production priffection of pertinent representations. While many (ilferent he design cycle, Bulding In tornation Modellng (BIM) is a methodology widely used to employ 'a digital re of a facility for design development, documentation and archiving (US national BIM Standard Project Committee). Depending on the size and complexity of a project, single building may be represented by a series
DiM Data for Supervised Learning: Data Pipeline

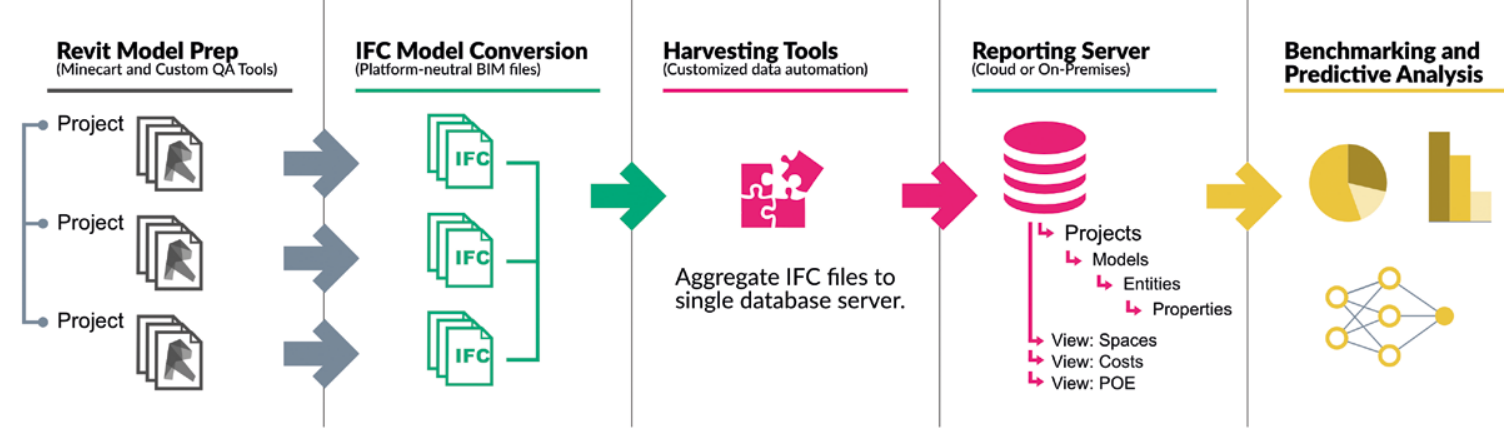




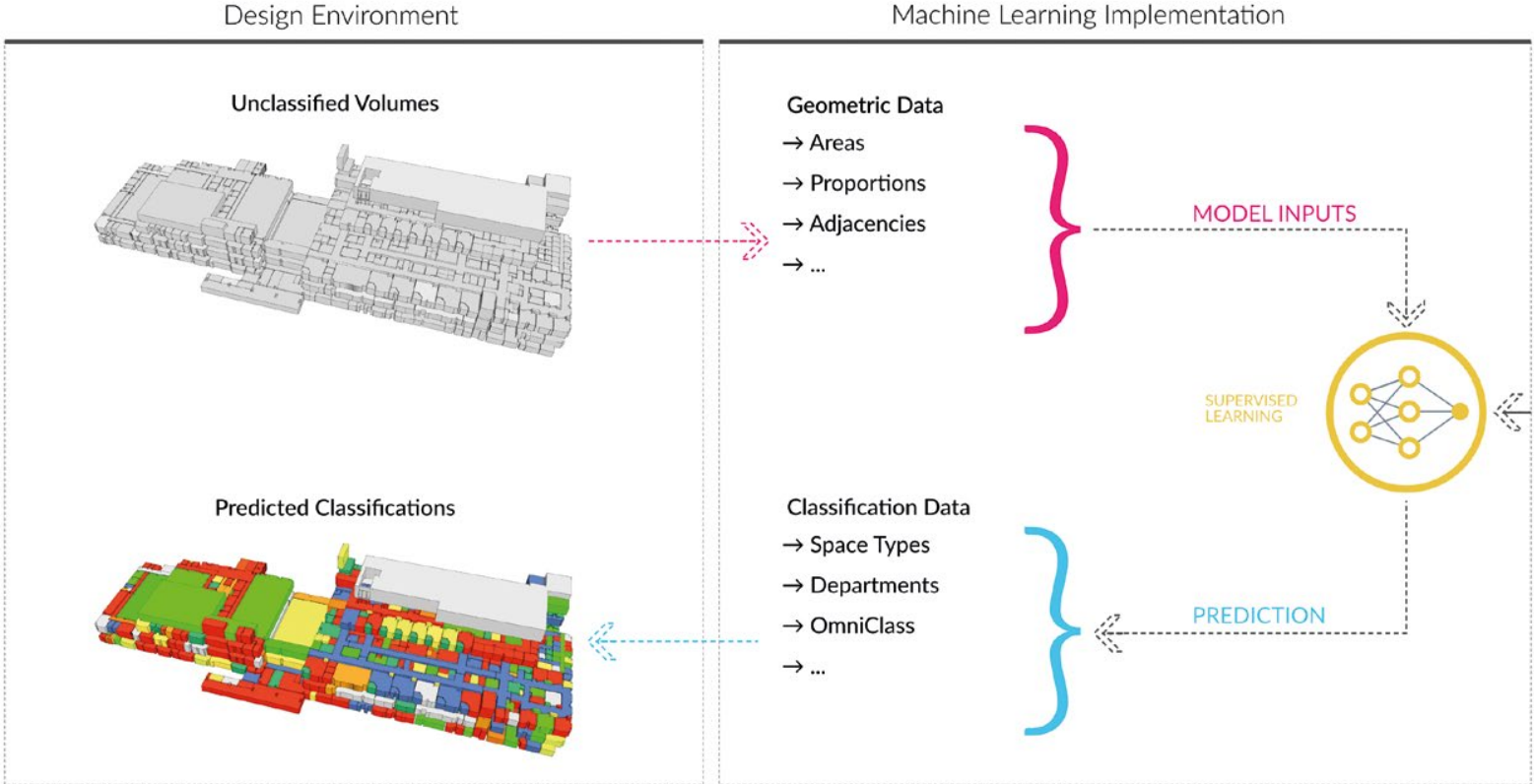

(sometimes dozens) of disaggregated model files, based on scope or discipline. Once their role is complete for building production, however, most of these models cease to be used in any way - with some notable exceptions for more sophisticated owners and operators, who may employ them for improved building operations. In the context of leveraging BIM for enhanced design intelligence through machine learning these historic models constitute an invaluable and largely untapped information resource.

Design of a Relational

Database for BIM Aggregation

We present a storage and access strategy that allows for data-mining models across individual and multip por data-mining (fig.1). Here, we have created and implemented projects (is 1). Here, we have created and implemented a relational database its population wh model data. The database is designed around the basic within Bum formats such as Revit and $\mathrm{EC}$, which has been extend to accommodate the information traversal that scales fromividur

The basic table structure hierarchy reflects this existing information infrastructure and intent for Elements-Parameters (PDEP):

Projects: Project information such as client, project name and market classification.

Documents: Document-level data such as file name location and file size.
- Elements: Individual object data, such as names, and categorical classifications, including walls, rooms and equipment.

- Parameters: Object properties with storage types for text and numeric properties, including for classifications, areas and quantities.

With a database schema established, tools have been developed to collect and prepare Revit and IFC file data, respectively, and which follow the processing pattern shown in figure 3 :

- File Harvesting: An automated routine processes a BIM file and establishes a dataset containing tables for document, element and parameter information. Harvester applications have been built both as an add- SQLFormanting: The processed dataset is ther automatically formatted as a series of text-file uploads to the database server.

- Database Upload: The data files are bulk-uploaded into the relational database tables.

\section{Data Harvesting for}

\section{Applied Machine Learning}

This harvester technology has been used in several professional architecture and engineering practices. This section focuses on its use at HDR Architecture, where it is deployed to support benchmarking analyses across several business sectors, comparing the spatial metrics of real-world projects relative to industry best practices and standards.

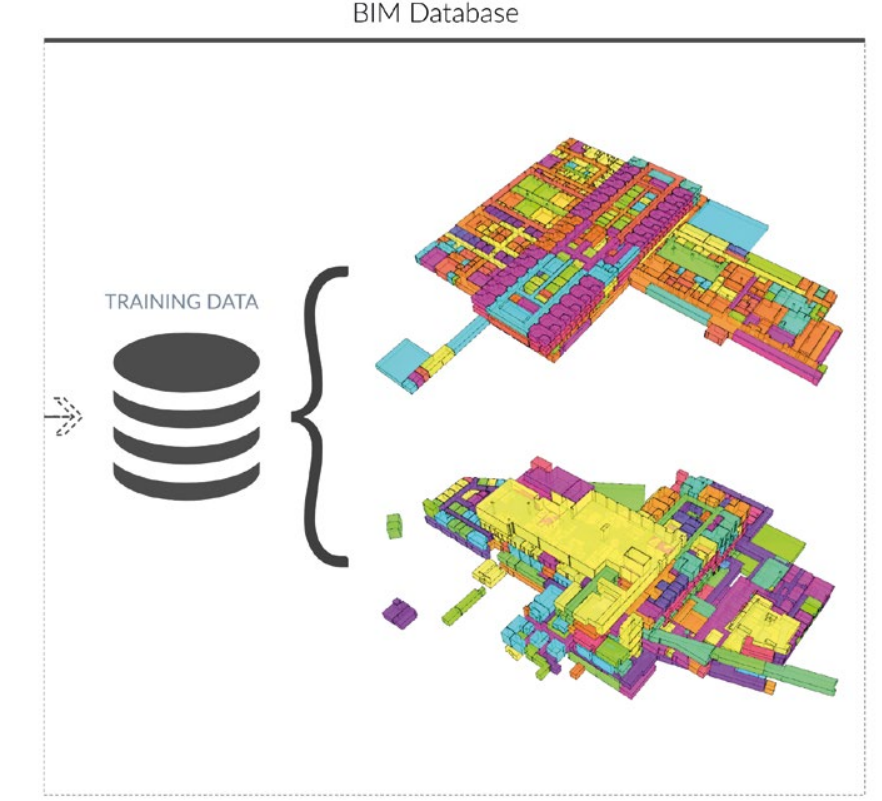

Here, we have created a proof-of-concept prototype that leverages data from three large-scale healthcare projects to train and test a machine learning algorithm (fig.2). (The limited number of models available for this prototype is a result of those made available for our collaboration; the setup is capable of handling thousands of models.) Planning for healthcare projects is accompanied by strict requirements, with individual spaces following specifications that include not only size and shape but also adjacency to rooms that house related functions, the inclusion of specific medical equipment and other considerations for patient and provider experience.

Historically, the design processes that aim to reconcile these demands rely heavily on a planner's experience and any heuristics they may employ, and such appric betime-consuming in broader application. This prototype positions machine learning as a support tool to facilt

\section{Predictive Modelling Prototype}

The learning model uses room-bounding geometry and building-level data to suggest viable Omniclass specifications whin the healthcare business sector? We hypothesise that, by training a model based on known prior room-programme assignment with a seres of descriptive features discernible from the harvested data, a supervised learning algorithm may provide architects with real-time critical decision design of a new building (fig.3).
Firstly, models in the database are evaluated for their consistency in employing OmniClass labels for room-level programme specification. This type of data-normalisation strategy is essential when project requirements and naming conventions for spaces vary between project teams and accordin to client requirements.

A first-order feature set is extracted from the database including room area, level, and department Grasshopper is then used to extract geometry from the IFC database to derive second-order descriptive features related to room shape and adjacency to other rooms, using spatia analyses based on 3D representations of each room. The OmniClass specification is then used as the labelled or predicted data feature. With these first- and secondorder descriptive features, two of the available IFC models are used to train a Naive Bayes Classifier (NBC) earning alcrith the the third model is resenved to test the viability of the a therithm. NBCs evalu categorich categorical input features to assign probabilites that A result of this is that test data points will frequently A result or this is that test data points will frequently have several possible outcomes, each attended by a NBC rili as deternined by the algorithm. Because B. res on categorical inputs for learning, continuous um erical feature data - such as area or aspect ratio of bounding box space - is prepared as a collection of categories (such as variants on 'small, 'medium' or large for room size). Based on this setup, our model demonstrates positive results as a decision support tool, with over $80 \%$ of spaces containing in their probable 

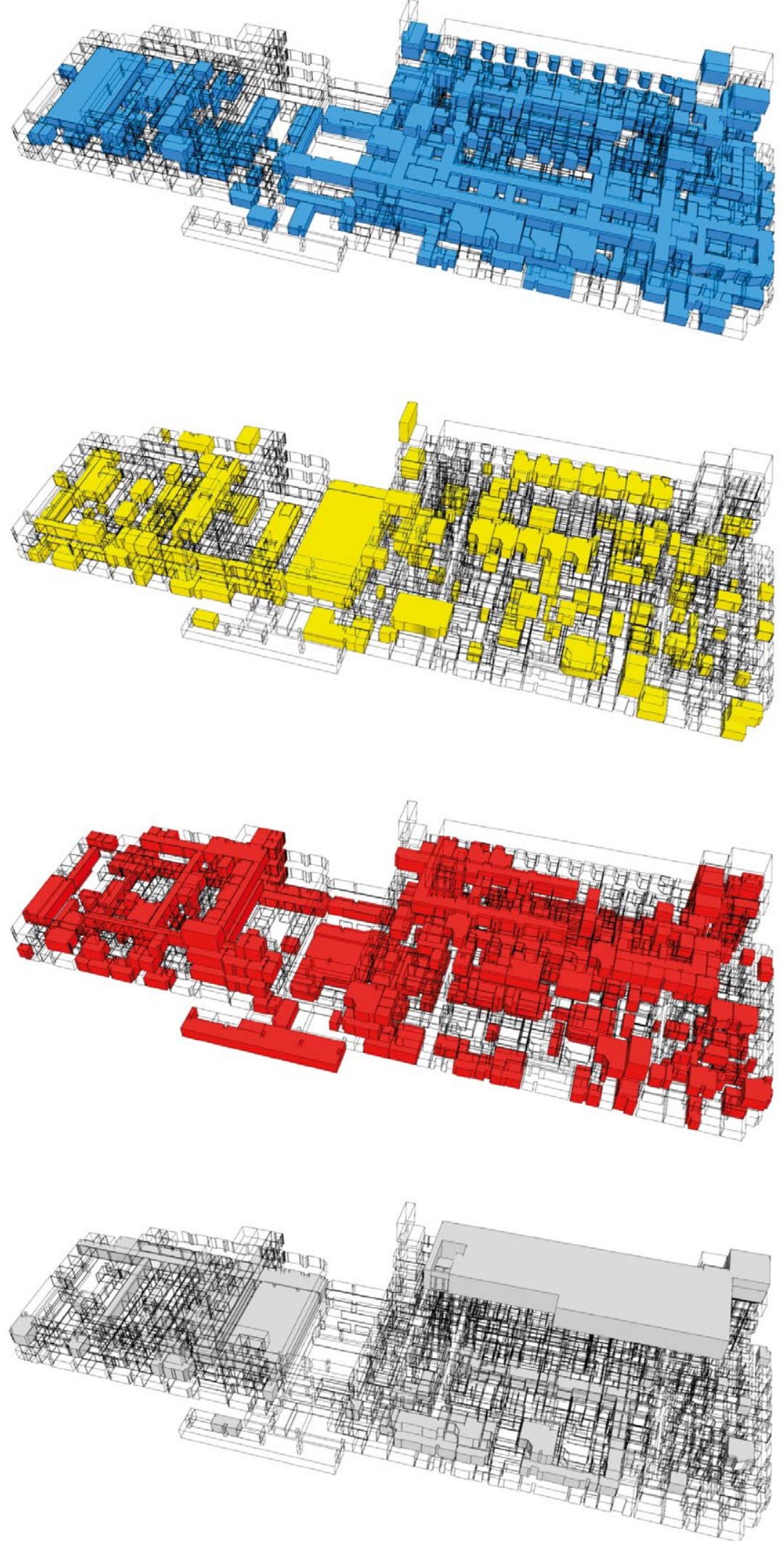

High Probability

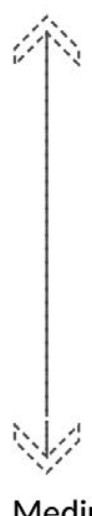

Medium Probability
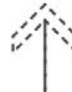

Low Probability

洲

No Classification
Building information models provide a consistent data structure that has been broadly adopted for production but is ultimately under-utilised for data analysis.

Our data harvesting techniques allow us to efficiently migrate BIM data into a scalable, centralised database that can be mined to train machine learning algorithm As these methods of data collection are adopted by building practices, we foresee both the need for greater accountability on the subject of data standards in order to successfully utilise the data for supervised learning, and also for the development and deployment of data remediation techniques for cleaning or repairing older data that may be of poor quality but still contain potentially useful insights for future design efforts. While this research presents methods and

technologies for leveraging building data, it also reveals challenges pertaining to data quality in the construction industry, which remains subject to inconsistent production practices, lack of standardisation, and error-prone data entry methods. Nonetheless, current trends indicate that the continued adoption of BIMrelated data standards and the growing market deman for data-rich digital assets should ultimately improve data availability for the enhanced training of machine learning algorithms for early-stage architectural design.
OmniClass is a North American construction classification . project-independent.

\section{Bibliography}

Brugnaro, G. and Hanna., S., 2017, 'Adaptive Robotic Training Methods for Subtractive Manufacturing' in Proceedings of the 37t Annual Conference of the Association for Computer Aided Design

Chaillou, S., 2019, 'Architecture \& Style: A New Frontier for Al in Architecture in Towards Data Science, 2 June 2019. (accessed 8 February 2020)

Davis, D., 2016, 'Evaluating Buildings with Computation and Machine Learning. ACADIA/2016: POSTHUMAN FRONTIERS: Annual Conference of the Association for Computer Aided Design in Architecture (ACADIA), Acadia Publishing Company, p.116-123. Derix, C. and Thum, R., 2000 'Self-Organising Space (SOS): Artificia Growing Neural Networks for Generative Urbanism', $10^{\text {th }}$ Generative

McGraw-Hill Construction, 2012, 'The Business Value of BIM in North America: Multi-Year Trend Analysis and User Ratings Construction Research \& Analytics.

Nicholas, P., Zwierzycki, M., Clausen Nergaard, E.,. Leinweber, S.,

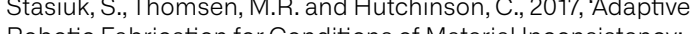
Increasing the Geometric Accuracy of Incrementally Formed Metal Panels' in Fabricate 2017, London, UCL Press, p.114-121.

Pena, A. 2012، 'Two Case-Studies of Freeform-Facade Rationalization' in Achten, H., Pavlicek, J. Hulin, J. and Matèjovsk a, Conference, volume, Vol. 2, p.118

Peng, W. Zhang, F. and Nagakura T., 2017. 'Machines' Perception of Space: Employing 3D Isovist Methods and a Convolutional Neural Network in Architectural Space Classification' in Proceedings of the Design in Architecture (ACADIA), Acadia Publishing Company.

Rittel, H.W. and Webber, M.M., 1973, ‘2.3 Planning Problems Are

Stasiuk, D.., Thomsen, M.R. and Thompson, E.M., 2014, 'Learning to Be a Vautt: Implementing Learning Strategies for Design Exploration England, CAAD Education, p. 381-390. 


\section{Research Summary \\ Multiple Criteria Design \\ Exploration in the Early \\ Design Phase}

Zeynep Aksöz

Industrial Partners: Foster + Partners, str.ucture GmbH

Academic Institution: Institute of Architecture, University of Applied Arts Vienna

Conventional design practice is often constrained to follow a linear path, starting with an early design sketch, leading to late-stage engineering resolution, design optimisation and execution that may run counter to aspirations defined in earlier stages. Indeed, some early decisions may in fact complicate 'more informed' optimisation procedures in later stages. Such flawed design-to-realisation pathways can be wasteful with an uneven distribution of time for the investigation of novel approaches and solutions.

As computational power increases and dataprocessing accelerates, the simulation of design performance also becomes faster. Accordingly the integration of applications for optimisation is moving to earlier stages of design. While opening new horizons for exploration and optimisation, these processes encourage an obsession with numbers and performance, where design is often mistaken for conventiona problem-solving. However, early design exploration is actually a stage in which the variables and goals that influence the optimisation and seach process are not clearly defined. This stage is, characteristically, a cycle of iterative exploration, evaluation and reflection, where the role of the designer becomes more about framing the questions than finding solutions.

This research project explores design methods and workflows that break the linearity of the traditiona design process, while still accommodating the flexible and ambiguous nature of creative exploration. Acknowledging the potentials of technological developments in the field, through the integration of heuristics and machine learning, the research investigates methodologies of collaboration between designer and computer to amplify the creative exploration. It asks how processes in the early design phase to negotiate between performative design objectives and aesthetic aims, and how this awareness can positively influence optimisation procedures in later stages.

Here, technology is approached as a communication medium rather than a decision-making tool. A new way of thinking is established, integrating generative processes early on. Design outcomes are approached as emergent products of a collaborative system between designer and computer. Design methods are developed that integrate machine learning technologies with heuristics. With this fusion, design-to-realisation processes are transformed into sustainable workflows, with information harvested across design processes and utilised to establish a computational tacit knowledge across projects. Through a feedback loop that integrates this knowledge in the early design exploration, designers are informed of future optimisation goals. The creative exploration is consequently, expanded into an information-rich process, where the performative gools rextive goals are gols to be defined and evaluated at he outset As a result a cross-disciplinary dialogue emerses between professionals and stakeholders to understand the nature of the design problem early on.
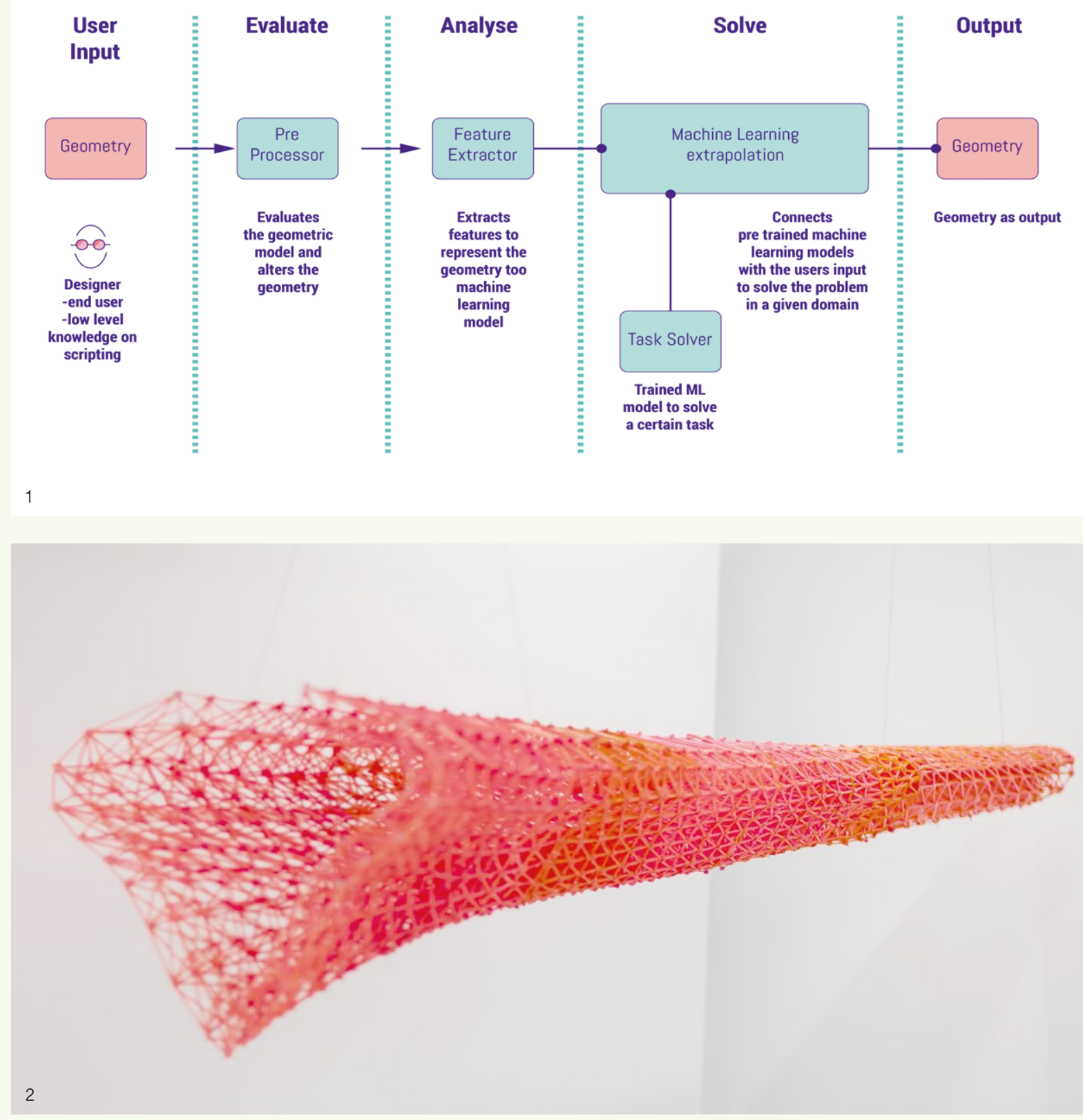
learning strategy instead of an iterative optimisation solver. Instead of a global he global parameter, this method solves the global geometry into independent modules that share the same topology. This way, an trained for a certain local problem can predict approximations of locally optimal
2. A locally optimised lattice using a local learning strategy. This prototype was the Foster + Partners and was robotically
Fost printed in their office. 


\section{Research Summary}

\section{Morphogenetic Fluid Dynamics}

Angelos Chronis

Industrial Partners: Foster + Partners, McNeel

Patonia (IAAC),

Universitat Politècnica de Catalunya

Today, performative morphogenesis provides common ground in avant-garde architectural research and practice. The form-finding design systems that, two decades ago, were envisioned to transform architectural design are now at the forefront of our built environment. Architects can now integrate most environmental forces that define a building's performance into their design systems, enabling them to determine their form. One of the fundamental environmental forces that affects how a building performs - as well as most form-finding methods in other fields - is, however, still largely unaccounted-for. The flow of air in and around buildings is, undoubtedly, one of the most important aspects of architectural design. As buildings continue to be one of the primary contributors of carbon dioxide emissions that climate change (UNEP, 2016), demand for cooling ene is expected to overtake that for heating energy in the coming decades. This project investigated the potentia of wind-driven form-finding in architecture aimed at optimising the naturat ventiltion potential of buldings. optinisive form- finding in archecture, amed at By inuegatic Computationa (CFD) simulation and shape-optimistion meds, explored how acrodynamic forces can dive he shape Over the course of the project, a number of integration methods for CFD in computational design were methods for CFD in computational design were developed and evaluated. Through industrial and academic collaborations, as well as workshops and other dissemination eftorts, the project exposed architects and researchers to CFD simulations and wind-driven form-finding. Using design experiments and case studies across scales, ranging from the cooling of the inner chambers of a 3D-printed clay wall (fig.1) to optimising the ventilation of the abandoned inner wind-driven shape-optimisation in the design workflows of architects today.

Input from the project's research partners - McNeel Europe and Foster + Partners - was instrumental in developing the tools and knowledge to direct the research efforts toward meaningful design problems. The project also established wider collaborations with international industrial and academic partners through the Innochain network, such as RhinoCFD, TU Graz and the host institute, IAAC, all of whom played a crucial role in developing and disseminating the research. Over the course of the project, 15 workshops, full-time seminars and courses were run, engaging more than 300 students, researchers and professionals with wind-driven shapeoptimisation techniques. Feedback from these courses, as well as interviews with prominent architectural practices and academics in the field, helped to shape the outcome. In parallel, longer-term research partnerships from projects at other institutions - such as the University of Patras, Greece, the Technica University of Košice, Slovakia and Innochain partner CITA, Denmak - senved as and sudies for testing the tools and methods developed.

\section{of this project has}

demonstrated that architects can apply wind-driven shape-optimisation techniques to augment the natural ventitation performance of our buildings and bulling components. The complexity of our design problems the various scales of engagement with airflows revealed that much work remains to be done to fully harness the potential of aerodynamic forces in architecture: nevertheless, we have he instruments to engage with these forces.

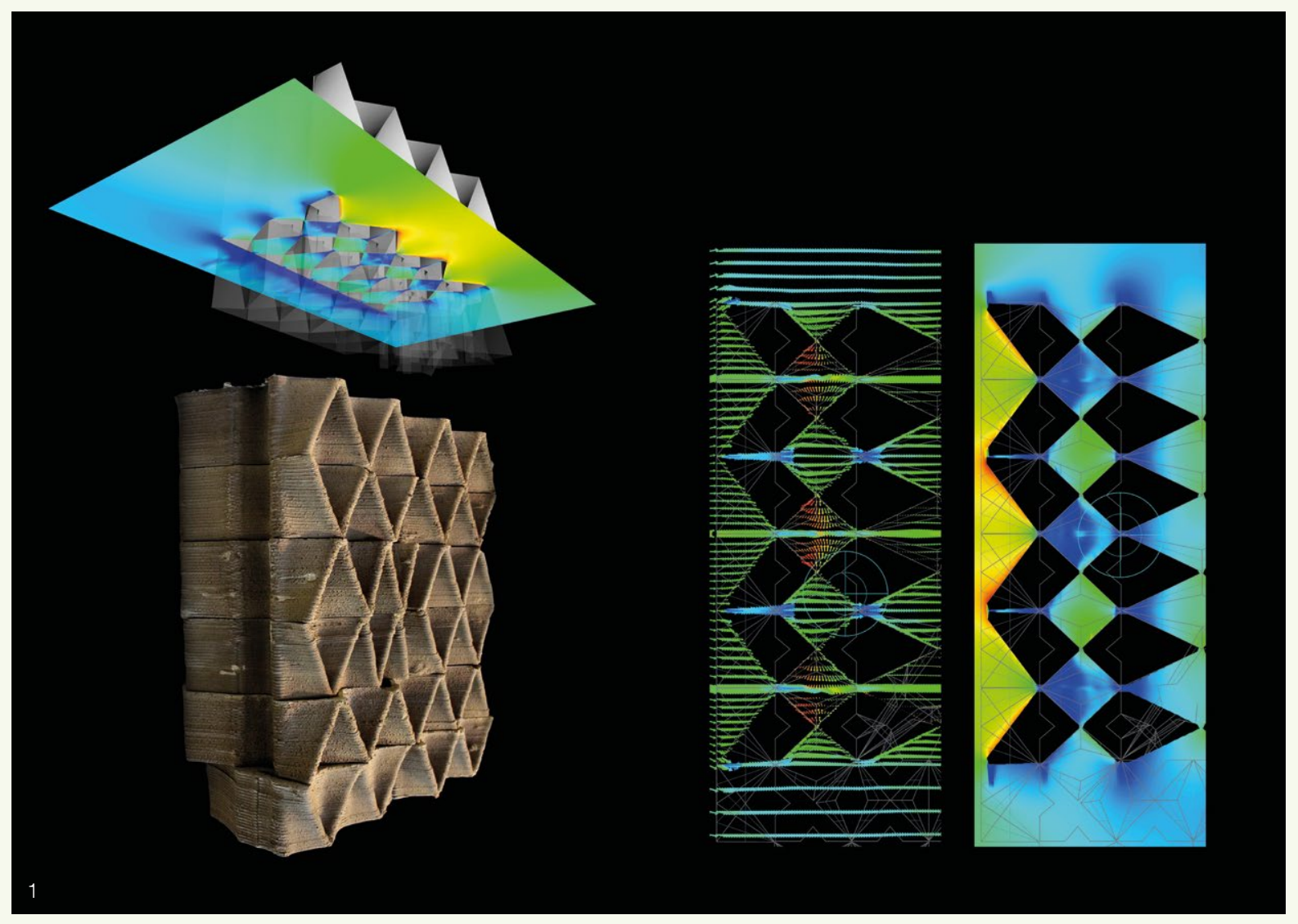

Bibliography

United Nations Environment Programme 2016, Nairobi, United Nations Environment 
Multiple States of Equilibrium for Bending-Active Tensile Structures

Evy L. M. Slabbinck

Industrial Partners: Foster + Partners, McNeel

Academic Institution: Institute of Building Structures and Structural Design (ITKE)

at The University Of Stuttgart

A combination of bending-active elements and a structural membrane introduces new integrative solutions into the design space of adaptive structures by using their potential for multiple states of equilibrium. The particularly complex nature of Bending-Active

Tensile Hybrid (BATH) structures affects most aspects of their creation, from modelling and analysis to fabrication, construction and detailing, restricting their adaptivity and the possibility of building them at larger scales. Changing the shape of BATH structures challenges the reciprocal structural equilibrium, namely the membrane and the bending-active elements. There is a need for a new and comprehensive approach to desisn and a new and compreh to The research was conducted on differentlevels. First, a literature review was conducted to give context; second, several concepts, strategies and ideas were generated and pooled to answer the following research guestions: "How are BATH systems designed with multiple states of equilibrium to utilise their potentiat in multive arching

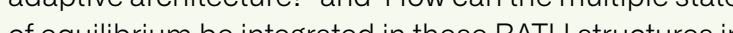
of equil brit be in the a ded in these BATH structures in such a way the the membran does notwinkeor break? to compre and examine the differest stacoses. The to compare and examine the diferent strategles. The results from these analyses were then embedded in the bull research projects. BAT_02, the IECH Research Demonstrator 2017-18, and the computational bamboo installation. Finally, these experiments were analysed, re-simulated and discussed.

The research was performed at the Institute of Building Structures and Structural Design at the University of Stutgart, under the supervision of Professor Jan Knippers. The Institute has a long history and is a leader in the research of innovative structural systems, high-performance materials and integrated fabrication processes. One of the built research projects, BAT_02, was developed in collaboration with the linked Innochain industry partner, Foster + Partners, which supported and helped frame the architectural context of the research.

The research opens new doors for adaptive architecture and BATH approaches in general, and is
a stepping-stone for further investigation and future potential applications. First, the structural systems show that it is possible to create large movements using small and simple actuation forces (ITECH Research Demonstrator 2017-18). Second the results indicate that structural membranes allowing movement are that structural membranes allowing movement are not a imitation of the system, but rather can act as a structur structures 0 beyond merely tubes and membranes, the also integrating plates, material gradient, combination of materials and so on making the design space larger and improving its structural properties (ITECH Research Demonstrat 2017-18 BAT The BAT-02 project begins to addess the need for The BAT.02 project begins to address the need for further development it holds the potential to be ap fur her development, it holds he potential to be applied plan in particular, regarding the link between is planned, in particular, regarding the link between construction, system typology and scale. In addition, steps have been taken to integrate the design-tomanufacture process of these structures so that all steps are thought-through in the intilial design and form-finding stage: material, actuation, construction,
1. BAT_02 at Foster + Partners' office, London. 2. Computational bamboo installation at the
Museum of Interactive Science in Quito.
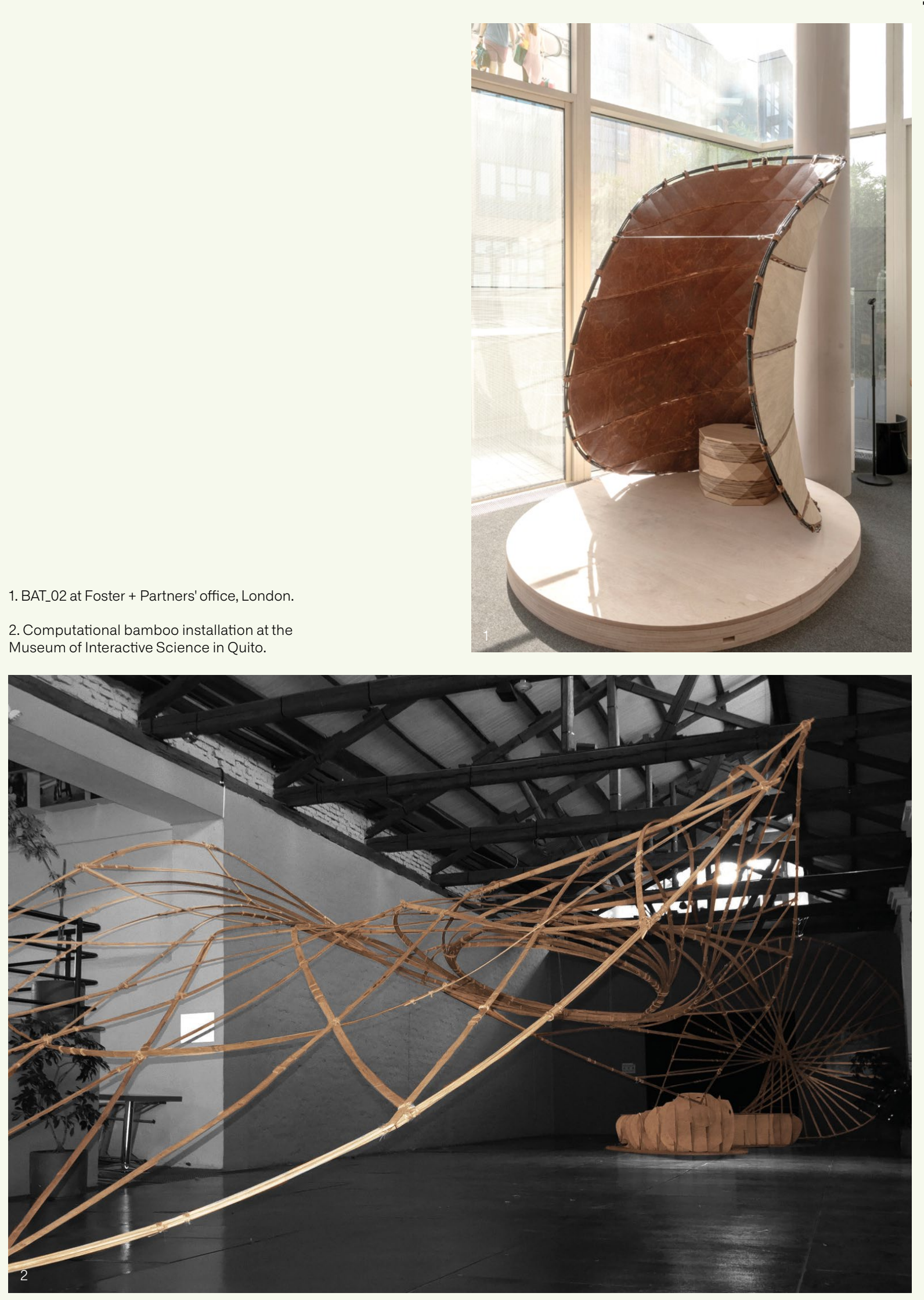
Research Summary

\section{Alternative Methods for Data Transaction}

Dimitrie Stefanescu

Industrial Partners: Henn Gmbh

Academic Institution: The Bartlett School of Architecture, UCL

This research introduces an integrated socio-technical analysis of communication as applied within the digita design process, which challenges the existing status quo of the building industry. Three critical aspects of a communicative act were explored: data representation, data classification and data transaction. Throughout the project, software instruments, 'Speckle', were developed to enable the research to be validated. By leveraging an expanding the industrial and academic network provided by the project's context, these instruments were

continuously tested and iterated-upon during a threeyear living laboratory composed of industry specialists. In 'data representation' the communicative performance of a low-level composable schema is compared to the existing industry standard. It is shown that the ontological revision process at a representation level is better served by the former approach, while the latter impedes meaninguu dias and whiles stakeholders 'Dataclassification' meanwhile, sugest an object-centric approach to data persistence. By comparing existing file-based exchanges to proposed alternative, it is found that an object-be proposed alternative, it is found that an object-based axc a reduction in ' $n$ ise' In to in buk, any design data that is communicated has an in buk, any dosign data that is communicated has an

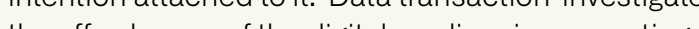
the affordances of the digt al medum in supporting communicative contracts between actors hat are revent to the organisational nature of the archite engineering and construction industries. design process can be attributed to the nature of its communicative processes and their subsequent distortion due to inadequate technological demonstrates that digital technologies can embrace the diversity and richness of the design process, enhance the collaborative aspect of the industry and open an accessible pathway toward a digitally-integrated built environment.

The project was awarded a $€ 1$ million follow-up grant, 'AEC Delta Mobility', by Innovate UK. Based on prior work on Speckle, the consortium - comprising The Bartlett School of Construction and Project Management, UCL; BuroHappold; 3D Repo; and Rhomberg Sersa Rail

Group - aims to define an industry standard for desig

change specification that allows faster and more agile 'delta' updates (in which only code is updated) to replace the file-based exchange mechanisms that are currently prevalent in the AEC industry.

Furthermore, Speckle was used as a technological base for research in participatory urbanism at the Future Cities Laboratory at the Singapore ETH Centre. From an academic perspective, the findings of this project can be used to inform further research in digital design collaboration construction and project mation realm of data interoperability in AEC.

At the time of writing, Speckle continues to exert a growing influence beyond academia, on the professiona practice of architects, engineers and other stakeholders involved in the design and construction process. It is incorporated into the digital transformation efforts of a number of international AEC companies, among the most prominent of which are HOK SOM, Arup, Woods Bet, BVN, Aurecon, DIALOG and Grimshaw Architects.

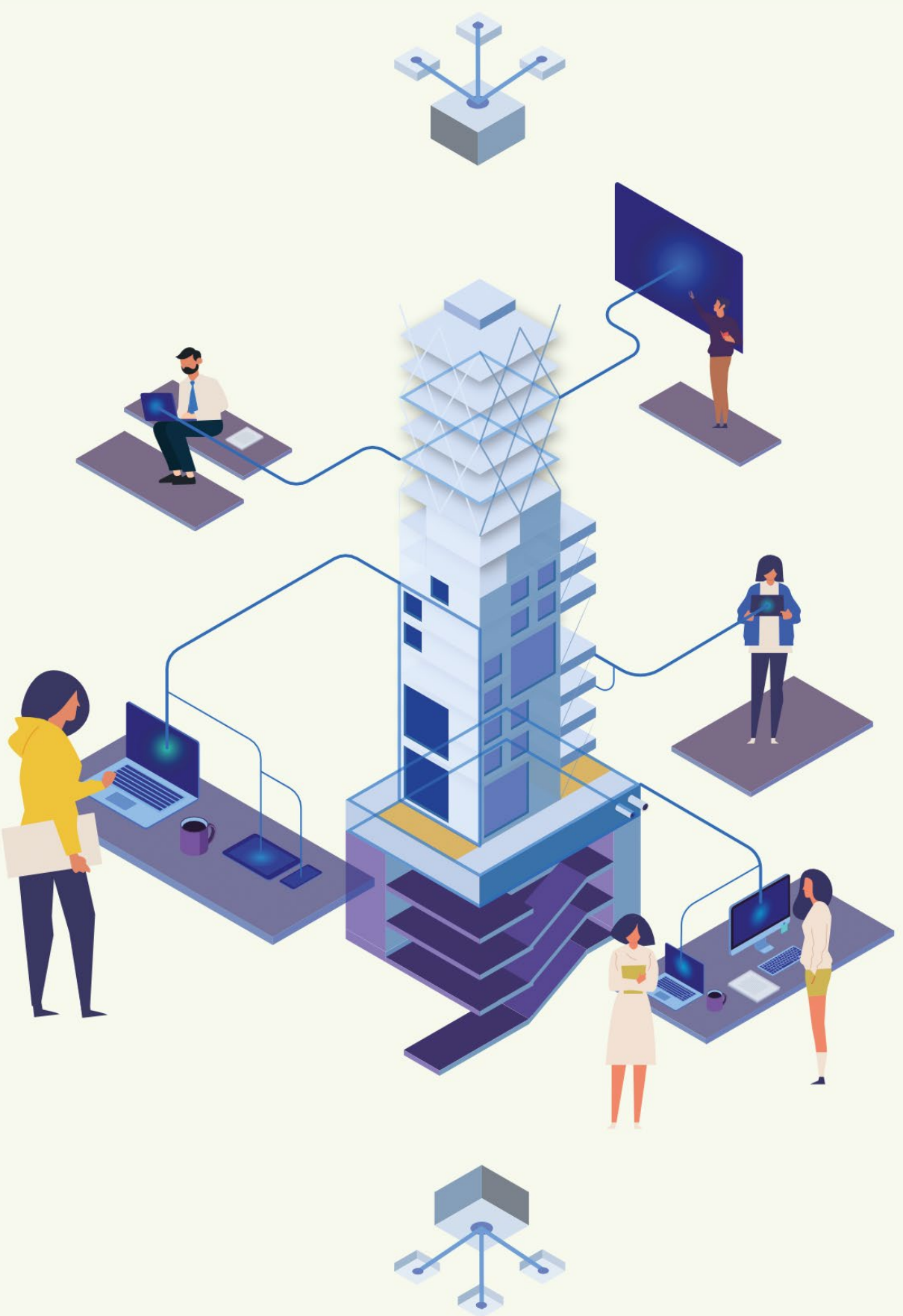




\section{Research Summary}

\section{Integrated Material Practice} in Freeform Timber Structures

Tom Svilans

Industrial Partners: Blumer Lehmann, Dsearch at White Arkitekter AB

Academic Institution: Centre for Information Technology and Architecture (CITA)

The Royal Danish Academy of Fine Arts, Schools of Architecture, Design and Conservation

Timber as a building material has acquired new relevance, not least in view of the climate crisis and concerns regarding overpopulation. Following new developments in adhesives and process technology, this sustainable, renewable and carbon-storing materia has made a return to the forefront of construction. This project examines the design and fabrication of large-scale freeform timber structures by proposing an integrated material practice in which design intent is informed by material and fabrication constraints, and new potential for architectural design is explored. The significant shift toward automation and prefabrication by the timber industry opens up a new ability to fabricate components with increased precision and complexity at larger scales, and with greater production volumes. Concurrent developments in engineering and material sciences have enabled the performative capacity of timber to be pushed further and perploited more thoroughly. While these devel further and exploited have hor have resul structures, engaging with fabrication affordances in early design stages remain a challenge. Similarly, the design and engineering ef freeform timber compenents challenge current

This project was developed in collaboration with Dsearch - the in-house research lab at Sweden's Wh Arkitekter AB, focusing on integrating computational design strategies within multilsciplinary architectura practice - and with Blumer Lehmann AG - a leading Swiss timber contractor speciallsing in the planning, development and delivery of complex timber structures. This threeway collaboration positioned the project production. The methodology drew on embedded secondments with industrial partners, materia prototyping and the interplay between designmodelling and fabrication. The research was tested and implemented through architectura competition proposals and ongoing design projects in practice.

The focal point and subject of inquiry was the glulam blank: glue-laminated, near-net-shape largescale timber components. A shift from a subtractive approach in timber processing to one of aggregation, through the development of structural adhesives, has opened up a large field of possibilities for composing precisely-tailored blanks, in response to design or performance criteria. From an architectural and design perspective, the space that the blank occupies between sawn and graded lumber, and the finished architectural component - holds much potential in yielding new types of timber components and new ilelding new typos of tring therefore, required new interfaces for design modelling therefore, required new interfaces for

The project created new ways of augmenting The project created in ways of augmenting existing design-modelling tools with lightweight material and fabrication-specific information. Deployed in early design stages, these revealed the production implications of design decisions, and allowed an interplay between early development and material performance. By integrating 3D scanning as a key component in the design-to-manufacture material calibration in fabrication. This created a digital material calibration in fabrication. This created a dig al link between fabrication data and material reality, during an encoded awareness of material behaviour

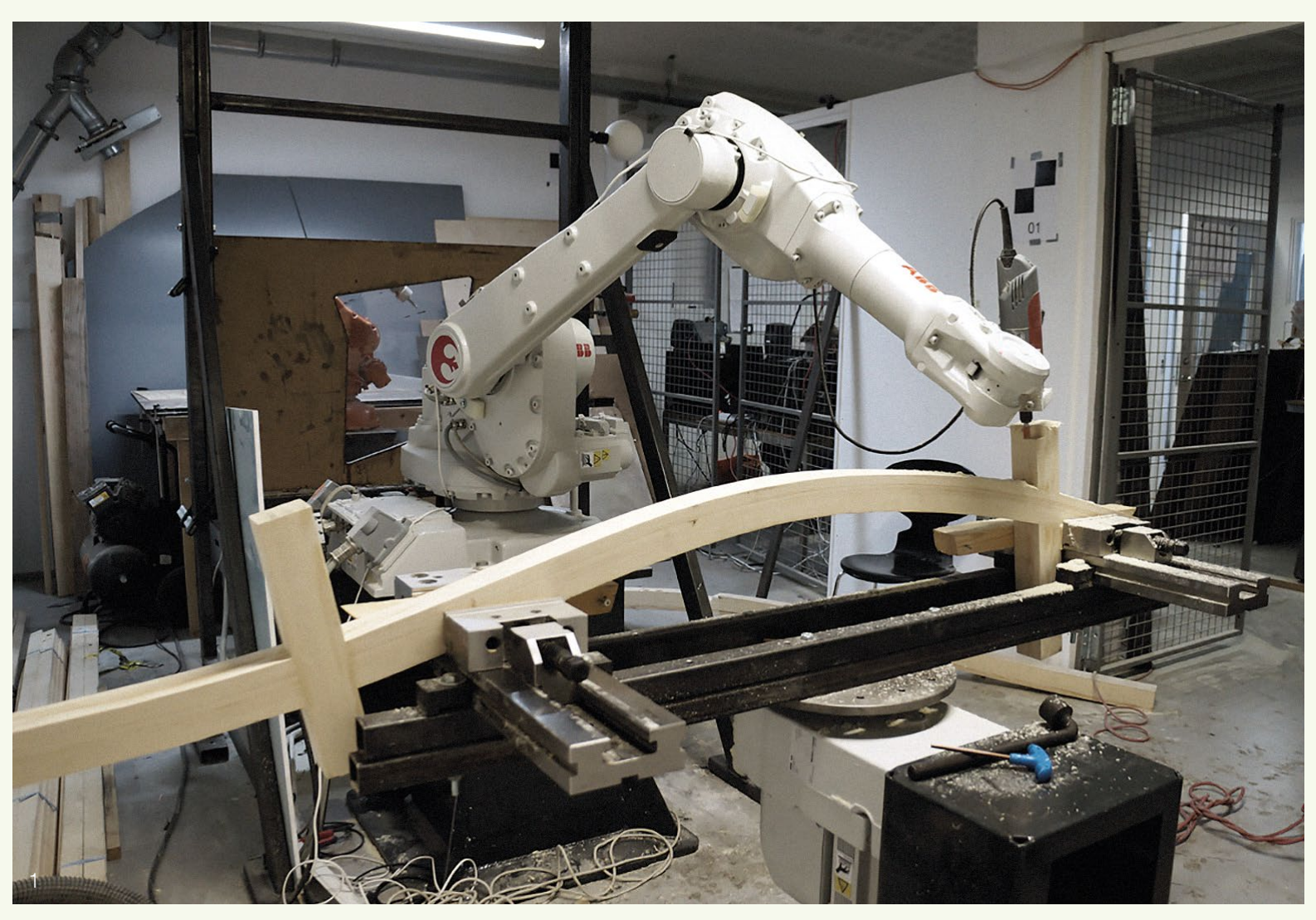

influence how architects and designers may interface with the material and fabrication constraints of engineered timber, and challenges existing industrial workflows by proposing a re-sequencing of the fabrication process and the integration of digital feedback systems. By suggesting new means by which timber architecture can be built, it also presents fresh perspectives on what timber architecture can be how can be shaped and what spaces it can engender.

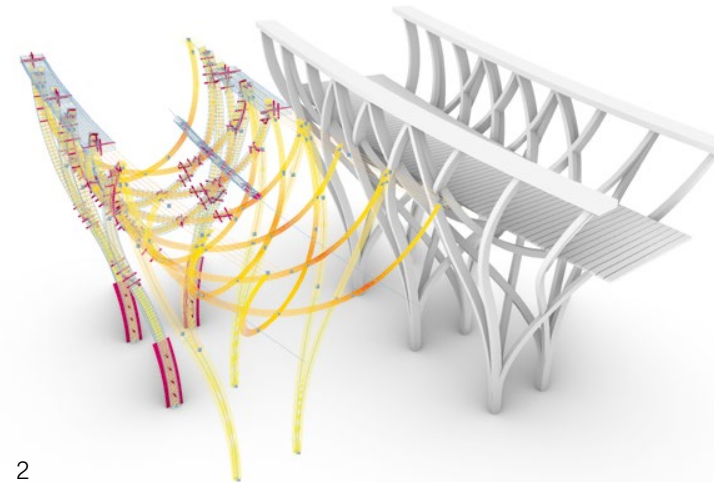

1. Robotic fabrication of glulam prototypes.

2. Augmented modelling tools integrate material prope
specification.

3. Integrated 3D scanning in multi-axis

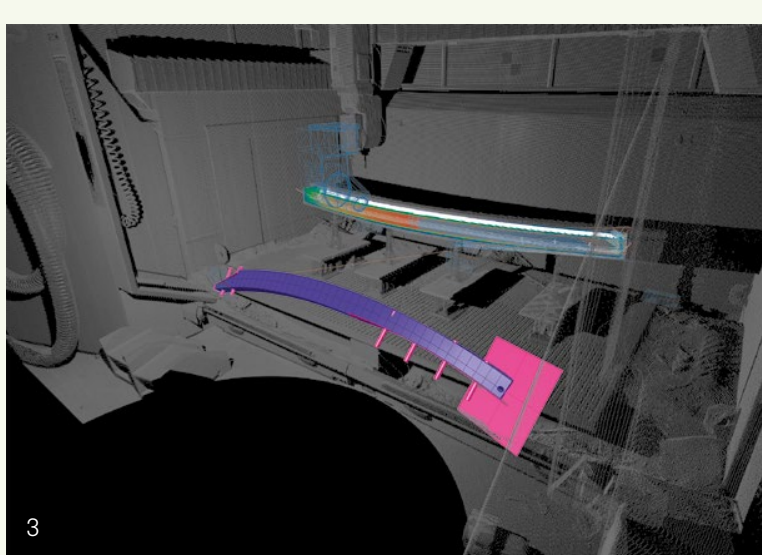




\section{Further Perspectives}

\section{New Workflows and Collaborations}

Kenn Clausen

3XN Architects GXN Innovatio

Sean Lineham

ARUP

Morten Norman Lund

XN Architects GXN Innovatio

Line Rahbek

Dorte Mandrup

Kåre Stokholm Poulsgaard

3XN Architects GXN Innovation

\section{Introduction}

As design modelling evolves, new tools and approaches emerge and, with this, data-driven modelling can integrate the work of interdisciplinary teams. As platforms and approaches proliferate, they pose new challenges to architecture studios. Studios have to integrate tools within workflows that are flexible enough to balance the emerging requirements of the different digital specialisations and the need for interdisciplinary collaboration. As the projects described here show, current practice puts significant demands on the establishment and maintenance of dynamic data models, as modelling practice expands from data models, as modelling practice expands from being cf collaborative workflows (Garber, 2017).

\section{Dorte Mandrup}

In 2008, while working for Zaha Hadid Architects, Line Rahbek found that Building Information Modelling (BIM) software was being challenged by the geometry of building designs andithad become necessary to move between difens setwe best results. While these er at the time, a few years later, similar work Hows were being bult into the leading software platiorms. The effor invested was rewarded, but often the palh was not directly from A to $B$. Thinking outside the box,

The Oecame a necessary tool for success. The Opal IDA project - designed by Dorte Mandrup for the Danish Society of Engineers - is a suspended structure on the Copenhagen harbour front. A freeform landmark, it has an almost weightless quality to its generated on how the different contractors could take drawings. To control the freeform geometry and also to share information with Seren Jensen Engineering Consultants, the building model was based on a 3D-point set via high-speed data-transfer (Flux). The point set was the basis for both the architectural design of the façade panels and the engineering design of the structural elements, with both sharing a common naming convention (fig. 2).

The Icefjord Centre is a visitor and research centre on a UNESCO-protected site in Ilulissat in Greenland. Its structure uses gently-tilted frames that blend into the landscape as a path. The complex twisting shape meant landscape as a path. The complex twisting shape meant Consultants was an advantage as it helped maximise precision and efficiency. The dataset - consisting of frecisorm curves - defined the geometry of the buildin and the movement of its edres, and was as important the tradition building

Some of the new workflows, such as cloud Some of the new workflows, such as cloud sharing cystemising through cloud-based parametric-driven and the Icefjord projects.

3XN Architects and GXN Innovation

The new International Olympic Committee (IOC) headquarters opened in summer 2019, designed by 3XN Architects. With its design for a dynamic façade, the building will look different from all angles, conveyin the energy of an athlete in motion. Each element of the façade needed to be unique in its shape and its

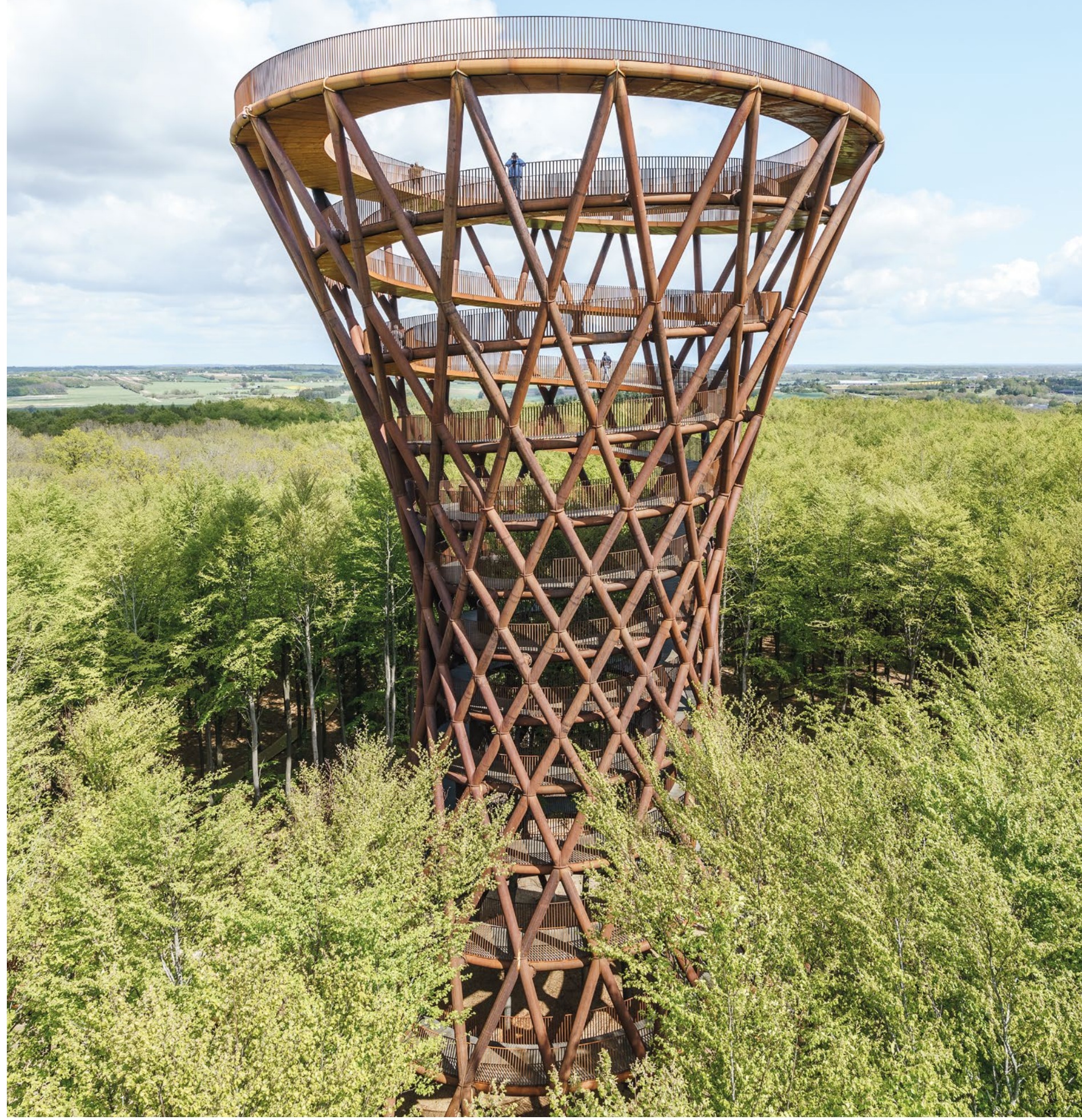

1. EFFEKT - Camp Adventure Tower.
Image courtesy of Rasmus Hiortshøj- COAST 


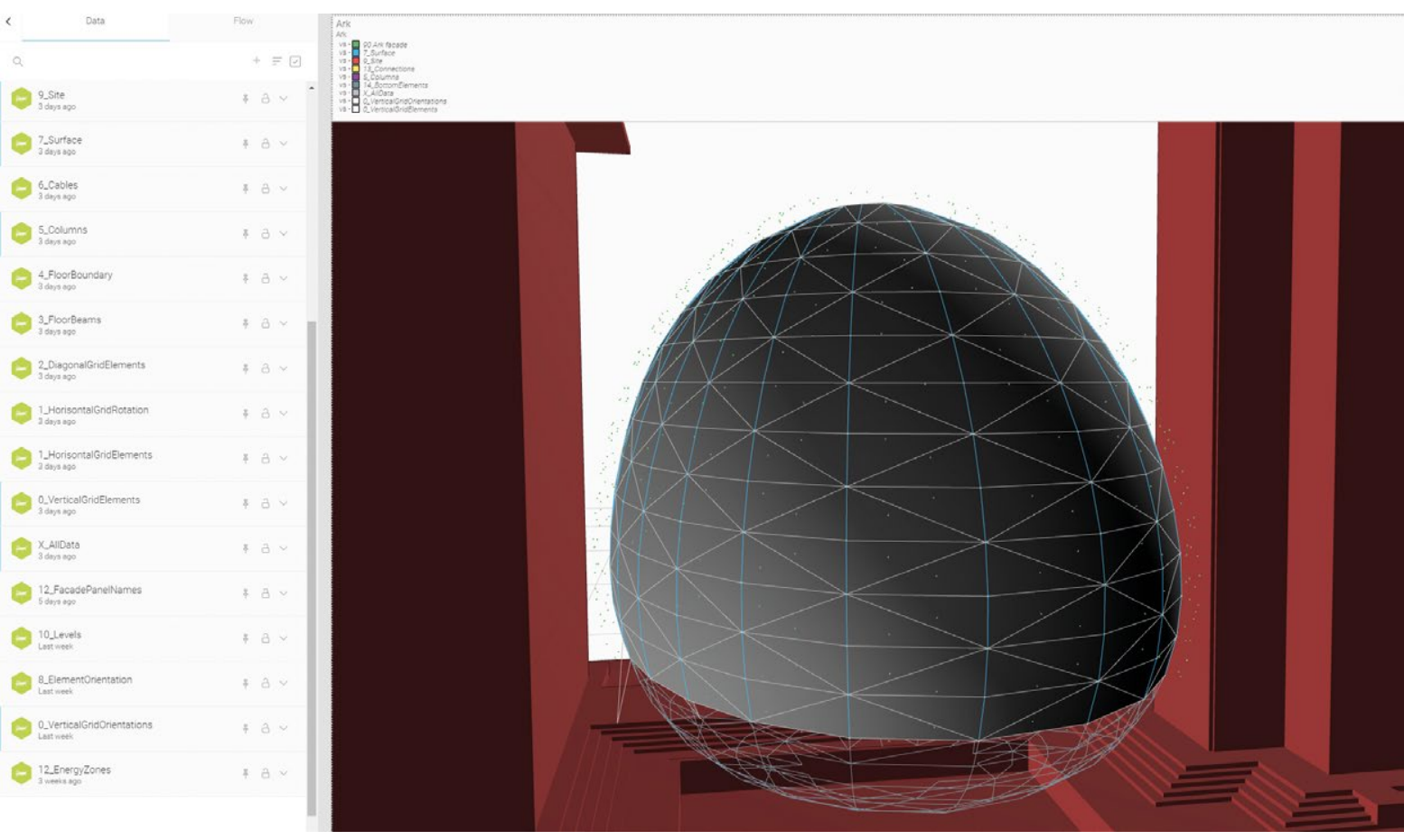

significant challenges for the $3 \mathrm{XN}$ team and their collaborators during design development. Iterations could have unpredictable consequences and affect geometric integration of elements, structural integrity of the full façade, aesthetics and building performance. To understand and manage relationships under strict deadlines, the $3 X N$ team needed a robust digital workflow that could tie the expertise of design and building information modelling into an efficientlyworking whole. GXN's Digital Design team established a data structure linking the façade model across Rhino and Revit, using Grasshopper. Dynamo and scripting to create a two-way data link. Updates in one modelling environment could continuously feed into thodelling enabling the different members of the project team to work simultaneously and efficiently arose team The efficiency of the data structure relied on a grid The efficiency 3 KN and GXN teams to ide that allowed This This shift toward control of work flows via an explicit dat building formation during design dexclonge of building information during design development. It also enabled collaborators to work on problems at diferent faç façade engineers could solve localised structural issues by inputting data on boundary condtions and constraints while $3 X N$ managed the integration of these, in keeping

4. Dorte Mandrup - IDA - The Opal. Screenshot from Flux, a cloud-based
platform. The image shows the grid controlling the freeform on which bo geometry.

5. Dorte Mandrup - Icefjord Illilissat. iM model illustrating some of the information you would extract from

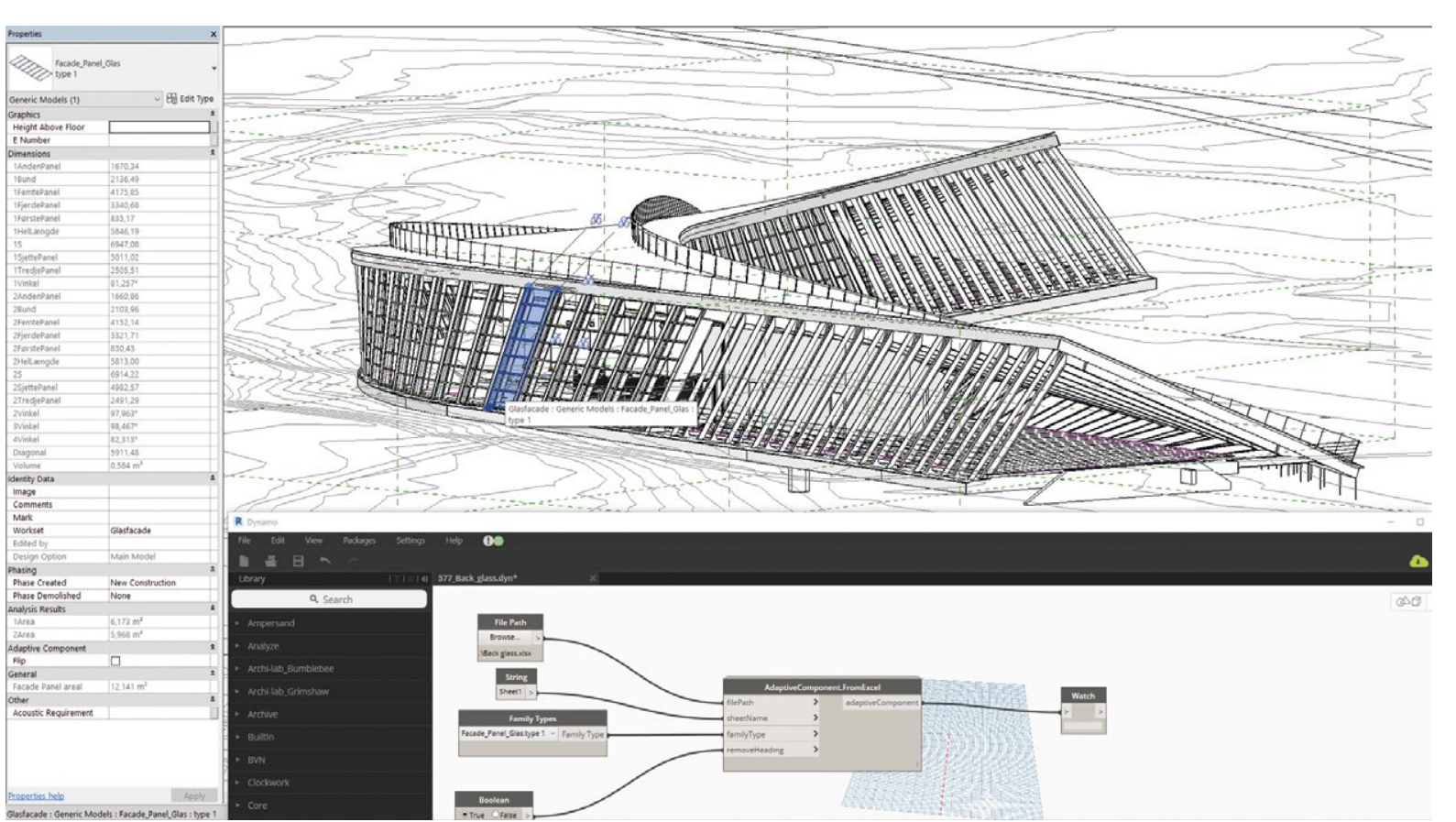

Data-driven collaboration proved to be dynamic and relatively platform-agnostic, as the methodology was applicable to different project and software environments in the studio. The model expanded from an environment for iterative-design exploration to a data-driven setup that serviced project partners with divergent requirements. The $3 \times \mathrm{N}$ team retained control of design and geometry by using data to structure collaboration with partners through all phases of design development and construction.

Arup

Digital tools and new approaches to workflows open fantastic opportunities in the building-design process. They allow us to They allow us to create ind explore design in new ways and can aid the collaborative effort between engineer, archict and contractor. Using parametric design actively with he architocture and the overarchand conctive A strong casestudy on

Astrong case study on workflows and collaborations is the Camp Adventure Tower, a unique installation fesigned to enablevisitors to experience theprore forest at Gisselfeld Kloster, one hour south of Copenhagen. The development comprises a walkway that gives vistors access to the preserved forest, before ascending above the trees, up a spiralling $45 \mathrm{~m}$-tall hyperboloid diagrid tower designed by Arup and EFFEKT
architects (fig.1).
To deliver the novel structure, the project required closely integrated architect-engineer collaboration and design workflow thinking. The design was conducted using a fully parametric workflow in Grasshopper with an array of interoperable plug-ins. The degree of integration between structural analysis, design and delivery in the parametric framework was extremely high. The setting-out of the geometry and the architectensineer coordination was conducted exclusively in code: the architectural, was condric and enclusively in design principles were collectively defined in one single Grasshopper environment.

Collaborating in code and developing workflows Co clo consequent analysis models architectural depictions and BIM deliverables were accurate and perfectly

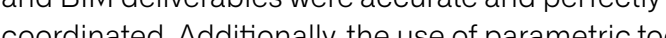
coor with a focus on atermining an option that met thatives, workflow was that it allowed more time for establishis the dow the detalls, such as the complex steel connections, and The success of the project was achieved by establishing a collective digital workflow for the main geometry early on, allowing time to refine and develop the detailing in coordination with the contractor. 
88
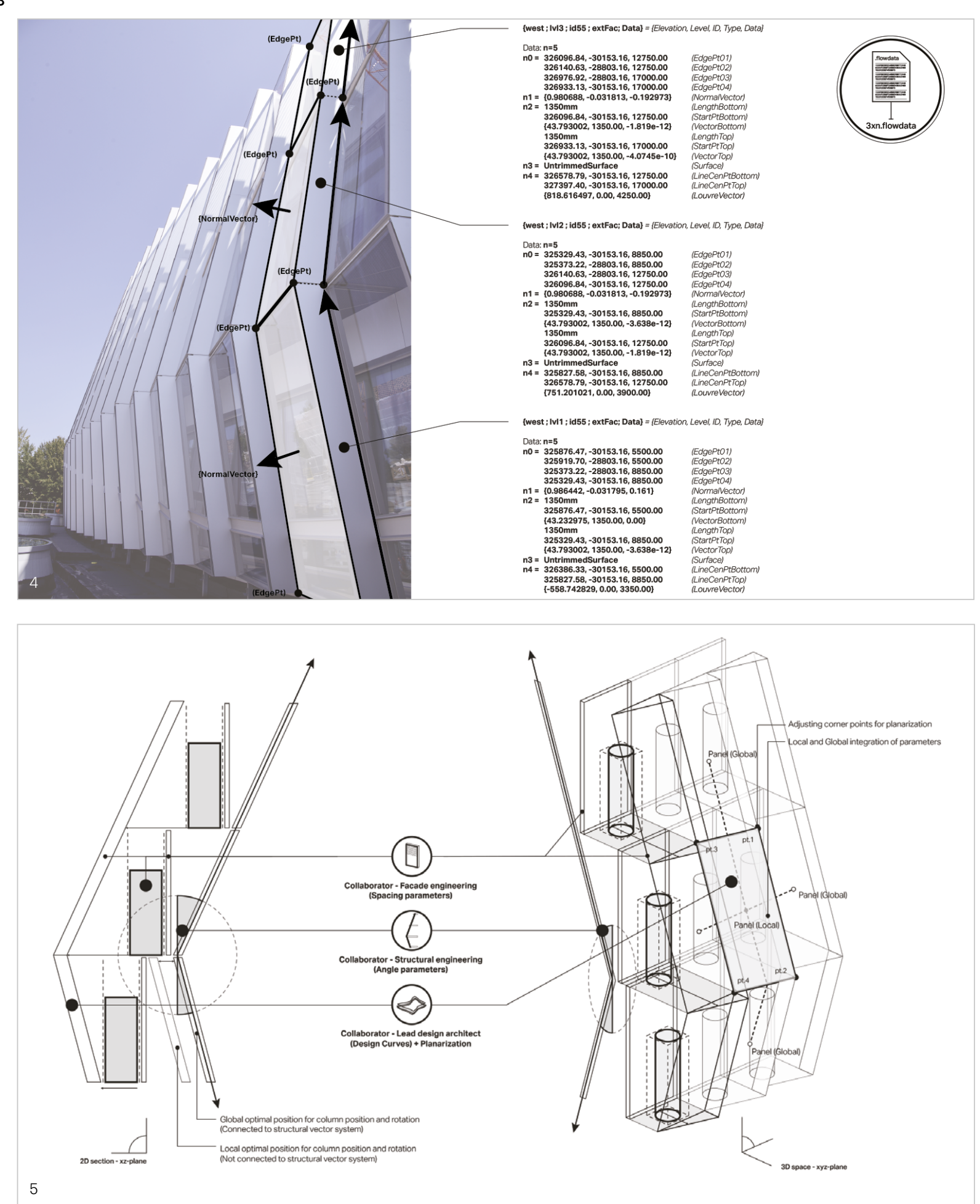

Promising convergence and greater interconnectivity (Deutsch, 2017), the current complexity of computational modelling means there are now innumerable information, software environments and practices. Scripting has empowered a new generation of architecture and engineering tool-users to become tool-makers (Burry, 2011), but the proliferation of approaches risks undermining the coherence of collaborative workflows as they expand in reach and scope.

Digital workflows and BIM provide teams with the ability to coordinate collaborative-working prior to, and during, construction (Garber, 2014), but integration between software environments remains challenging. The database/BIM standard is not yet structured and differs between offices and countries.

Interoperability and collaboration are core concerns for studios seeking to expand information modelling. As the quantity of information expands, it can be difficult to maintain quality and to filter relevant information within the model. Software has made these information-rich models attainable, but often by emulating older workflows and hiding data under user interfaces. Efficient methods for filtering and sorting data are urgently needed for close collaboration in interdisciplinary project teams.

The concept of BIM showed the way forward, and today the potential of integrating more datadriven approaches into practice is changing the way we think about our designs, communication and professional interaction with collaborators. Expanding information modelling promises a platformagnostic and dynamic approach to collaboration that no single software environment can offer at present. The strength lies in the data structure and protocols developed for individual projects, which enable dynamic exchanges and allow collaborators to work on globat design and construction-detaling simultaneousy.

\section{Bibliography}

Burry, M., 2011, Scripting Cultures: Architecture Design and Programmin

Deutsch, R., 2017, Convergence: The Redesign of Design,
Wiley \& Sons Inc.

Garber, R. 2014, BIM Design: Realising the Creative Potential of Building Information Modelling (second edition), Hoboken, NJ,

Garber, R., 2017, Workflows: Expanding Architecture's Territory in the Design and Delivery of Buildings, Hoboken,
Wiley \& Sons Inc. 

Design Integration:

Global Technological

\section{Advancement and Local Culture}

In dialogue:

Areti Markopoulou

Institute for Advanced Architecture of Catalonia (IAAC), Universitat Politècnica de Catalunya

Philip F. Yuan

Tongji University, Shanghai

Areti Markopoulou (AM): In an era of increased urbanisation, digitisation and exponential risk, the practices of digital design and manufacturing are expected to change not only our built environment and the business around it but, more than anything, the way we live and how we participate and interact within it. In this context, we discuss the applications and implications of integrating digital design and manufacturing technologies with strategies of sustainability that involve local

resources, culture and craft.

The impact of unsustainable materials and processes on the environment highlights the urgency for optimised design and manufacture. It provokes a rethinking of the

form and kinds of materials that should be used in

making our environments. Steel, concrete (cement)

and plastic are the building materials that dominate the construction industry today, with cement - which relies on coal and petroleum for its manufacture - being the most energy-intensive of all materials. The construction industry is the biggest generator of waste in these materials globally, and sustainable alternative material, design and manufacturing models urgently need to be established in the mainstream.

New construction practices and processes are being researched in which negative impact is reduced with a focus on natur resoures, advancements synthetically engineered zero-waste materials and an increased awareness of the importance of circular design strategies.' Alongside this, advancements in digital design and fabrication offer potential for materia, structural and construction optimisation, opening up possibilities for more sustainable, faster-to-produce

and, in some cases, cheaper constructs.

Techniques such as additive manufacturing can be 列 when needed, without the need for a mould or the creation of waste. Material libraries are shifting toward more sustainable, organic and natural consistencies, as well as natural materials found close to the construction site, which boosts new circular design strategies. Automation and the creative use of robots - industrial, mobile and aerial - play a critical role in this change, as do onsite, robotic manufacturing technologies and pop-up factories, as opposed to the expensive and environmentally costly modes of transportation needed for offsite construction methods. In this new material age, the architect-asprotagonist in the creation of urban, built and public spaces can guide us toward more responsible choices, integrating digital design with local material, culture and craft. Can each of these elements merge in emersent projects and research?

Philip F. Yuan (PFY): Key here is how we use traditiona craft and local materia Ken adopting new tools of design and fabrication. An example of how this process mishtberelised can be found in the ruta construction project 'In Bamboo', designed by Archi-Union and constructed by Fab-Union in Daoming Town, Sichuan Province, China, in 2017. A multi-functional rural community cultural centre with facilities for exhibitions conferences, dining and recreation, which integrates new construction technology with locally sourced wood and bamboo, and traditional construction techniques with prefabricated industrialisation.

The architecture, landscaping and interior were completed in 52 days, which was more rushed than initially imagined. By using digitally prefabricated structural wood components that were nested, volum transportation of bulk material was reduced and the
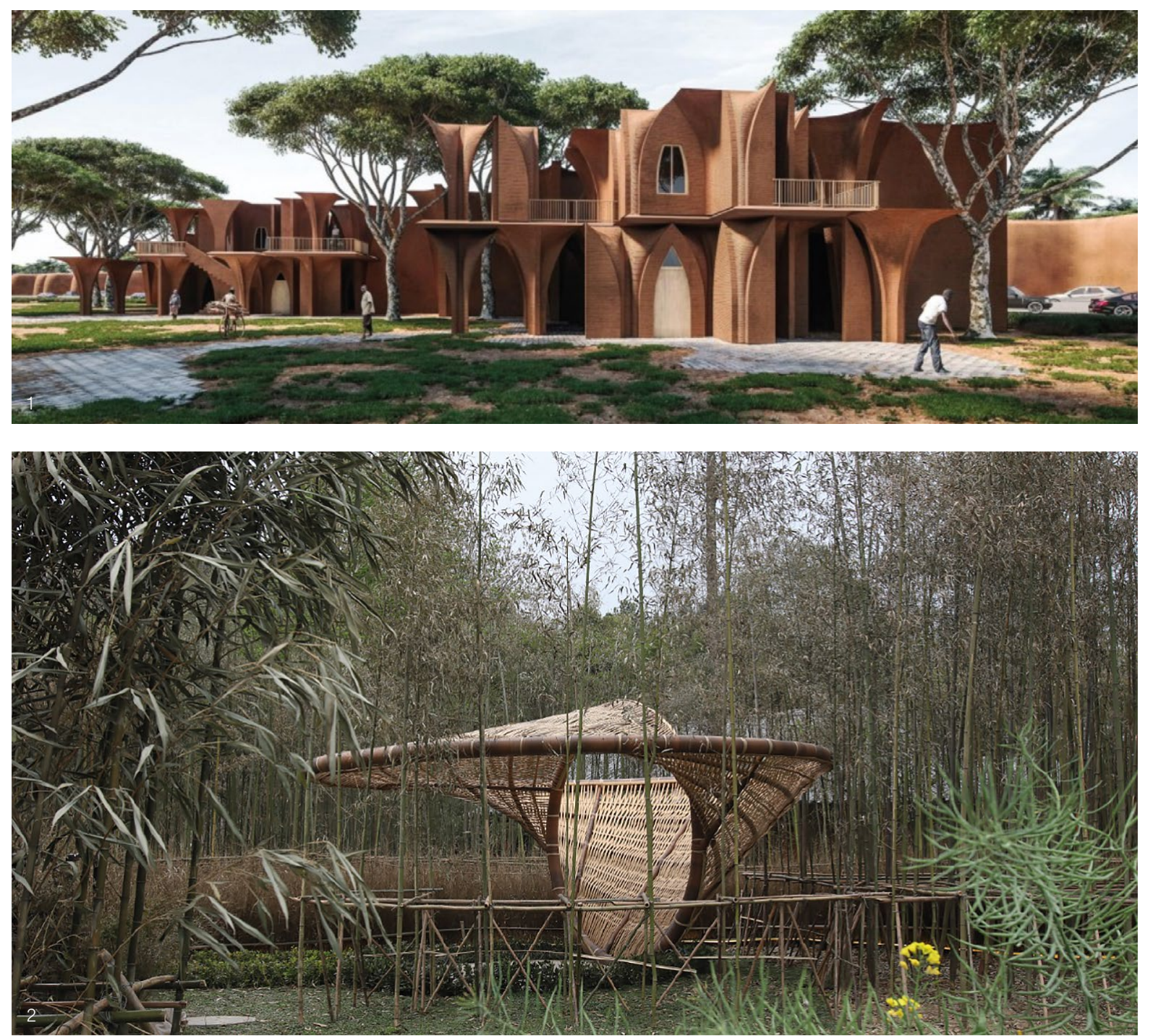

.3D-printed settlements made from locally

local users. Image: IAAC, Open Thesis

2. Weaving installation integrating digital

fabrication technology with local craft. 
94

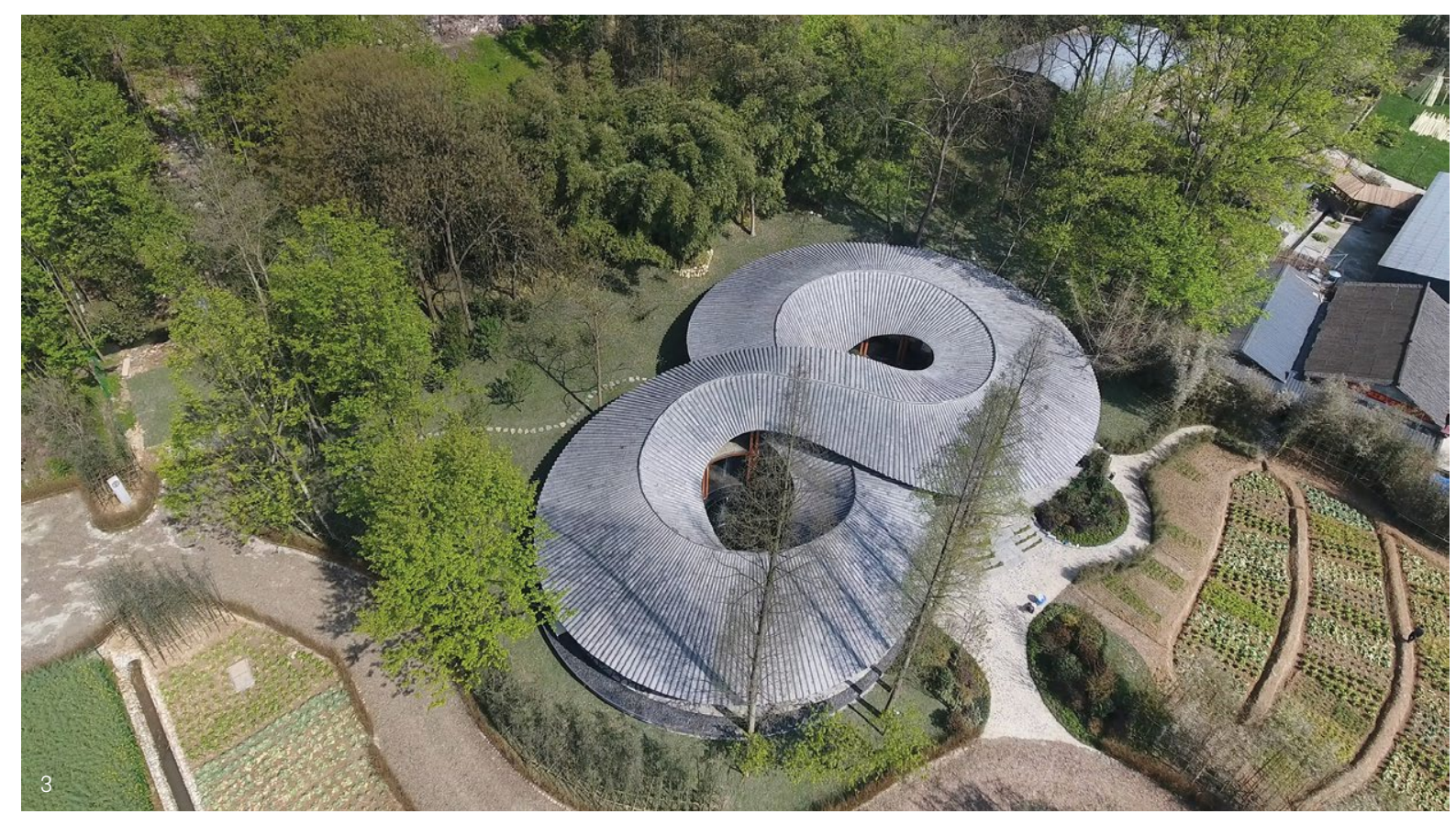

speed of assembly was increased. In the development of earth-based materials could provide solutions for this project, research from many years of experimenting with digital fabrication technology for wood structures was put to use at full scale. The gestural interweaving roof is a construction of prefabricated parts, delivered to the site ready for quick assembly. The Mobius-shaped roof is supported by a light prefabricated steel frame and is finished with local ceramic tiles. The efficiency afforded by pre-fabricating components made the creation of this complex geometry possible in the short construction period.

Such new modes of construction offer the possibility to reinvent traditional building materials and have challenged our understanding of a building as a fixed solid entity. From your perspective, how do transformable solid entiy. From environment to confront current global issus, such as the environmental crisis?

AM: In response to possibilities arising from a new digita and material age part of the research developed at IAAC and material age, part of the research developed at IAAC exploes the use of locally sourced, natural and recyclable materials for housing solutions. Earh-based materis are combined with 3 p pinting to producecomplex geometries for chmatic and structural performance using algonthmic design, and the potential of robotic technologies is used to improve the construction site using onsite robotics.

N Habitat, IAAC developed sustainable housing solutions on three sites in Africa that make excellent case studies. The collaboration urban flood protection (Suleja River, Nigeria) and sound protection during the construction of highways (Yaounde, Cameroon). Furthermore, the research has developed based on the engagement of residents with the construction process, as well as the possibility of responding to complex humanitarian situations with the new generation of social, economic and environmental capital.

IAAC's 3D printing projects use earth-based materials and provide zero-emission construction solutions while engaging users and local craft in their development. Robots are adapted for onsite construction, creating new possibilities for remote areas where resources are scarce Natural materials are combined with digital design and robotic manufacturing to produce digtal design and robotic manufacturing to produce contempory qualities with embedded performance that would Furthermous theen computationally simulated. Fin-pinted of 3 -printed earth architecture to adapt to siteFrom your expes, climate, conmunity and culure. From your experience, do you think that the use of locally sourced materials can bing people closer inform design and manufacturing?

PFY: Robotic fabrication and other new technologies could result in more meaningful and lasting changes for the rural construction industry. New technology ural life and industry; instead, we should consider opportunities for innovation and improvement that might integrate existing construction methods with new technology

China's rural industrialisation process is yet to begin, as urban gentrification and modernisation overwhelms the advancement of production system in the countryside. It is startling how difficult it is to find a worker in the countryside under the age of 40 , as the traditional architecture industry has no mean of attracting the younger generation. Right now, prefabrication for use in rural areas could redefine and upgrade traditional construction.

Daoming Town is well known for its enduring tradition of bamboo weaving, which is integral to how families spend time together and neighbours interact A traditional craft, it is also a living cultural heritage with much to offer contemporary ways of living and making. For In Bamboo, we researched local architecture and the limitations of using bamboo as the primary structure system. We learned that bamboo performs very well as a protective sheathing on the exterior façade of a building. Working with a local artisan, we modelled over 20 different variations of weaving patterns with thin strips of bamboo that could be used on the façade. This produces the experience of seeing something familiar but encountering it in a new context.

Industrial robots are the revolutionary construction platform of the digital era; offsite prefabrication technology and in-situ robotic construction not only give traditional craftsmanship a new identity but also
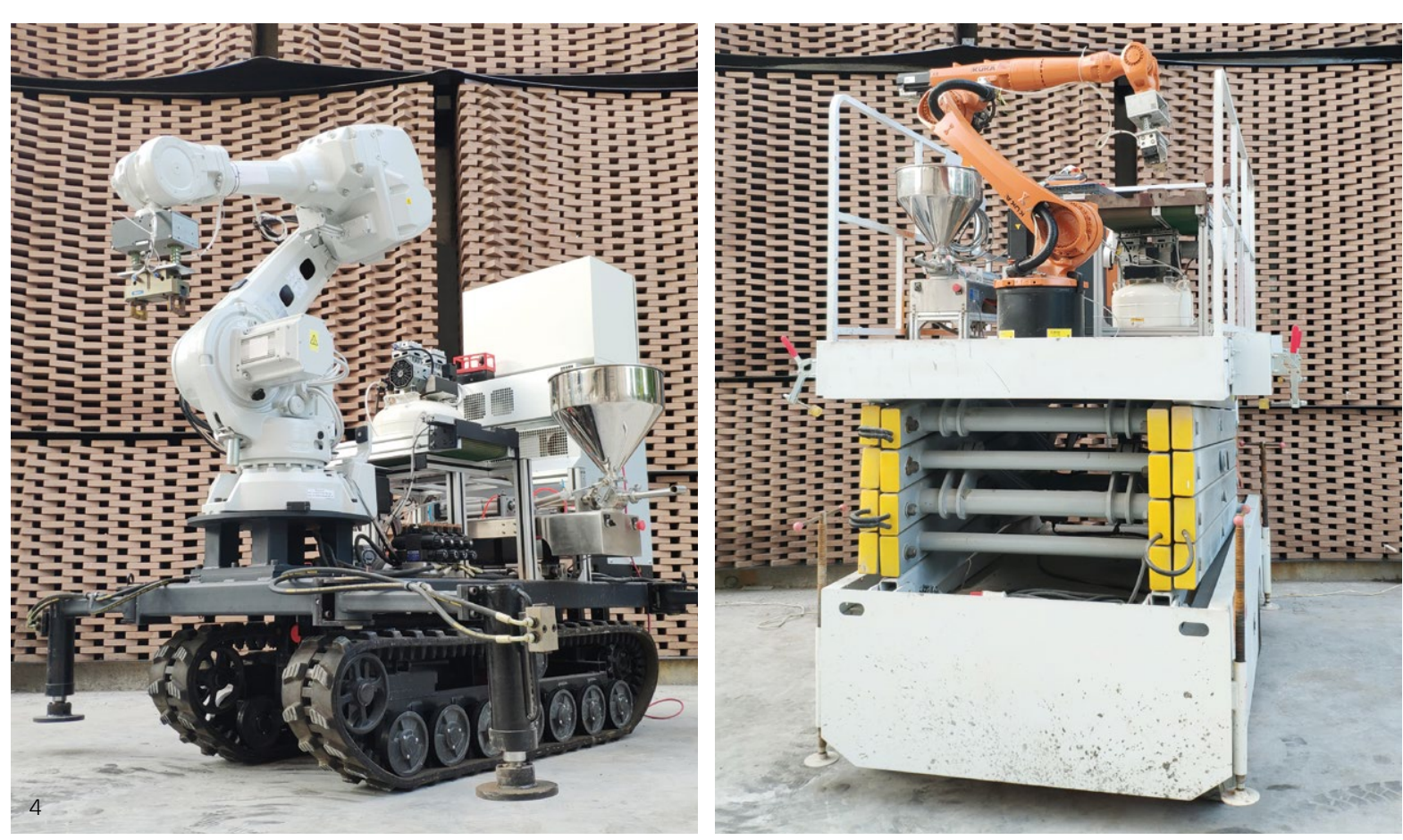
96

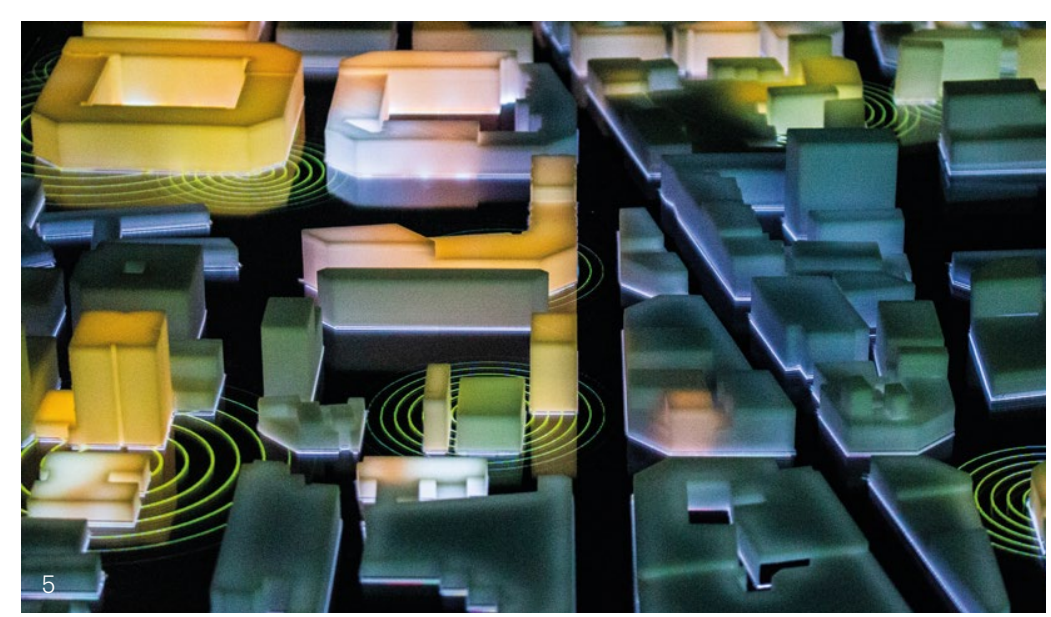

processing infrastructural datad buildings, and urban-planning scenarios. Image: IAAC City \& Technology, Internet of Buildings, 2019 .

6. Superbarrio is an open-source
virtual-gaming interface forcitizens to engage with public-space design.
Image: IAAC, City \& Technology, 2016 .

make open-ended mass production and customisation possible. In your view, how could our living environment be reformed by the responsive construction process, whic is highly sensitive to human beings, in both temporary and spatial dimensions? How do you think the public contribution could improve the current relationship between research and practice in our discipline?

AM: History indicates that any profound social change happens in circular feedback with a technological revolution, meaning that one is both fuelling and being fuelled by the other. According to the authors and researchers Erik Brynjolfsson and Andrew McAfee, the advancement of digital technologies is affecting mental power in the same magnitude that the steam engine affected muscle power. All agents involved in contemporary design, including architects,

manufacturers and users, are gradually shifting their modes of operation, inhabitation and interaction.

Increasing digital connections and the rise of Web 2.0 have boosted a highly participatory culture. More and more people are becoming familiar with the design and production of their surroundings. and customised manufacturing technologies enhance the posibilites for particin. Within this context, the possibities for participanion. Within his context, the architectural discipline encompasses new design and makng procescipe For a in Barcelona and Mum ba by IAAC, vitual gaming platforms and vitual realty technologles were used to engage citizens with the design or the public space and the new buldings in their neghourhood. Machine learning and Articial Intelligence (Al) were used to analyse data to create an urban simulator for more mformed decision-making processes in the urban environment. Similarly, in the 3D-printed earth project, users could customise the design of their home based on their personal criteria, as well as on the materia final form defined by digital simulations.
Similar to the effects of the Industrial Revolution, architecture in the current digital age is evolving to become a natural nexus between bits (digital world) and atoms (physical world), while cognitive decisions, cultural aspects and crowd wisdom merge in a unique way with computational simulations, predictions and manufacturing processes. The new design paradigm emerging in architecture promotes novel design processes in which designers, users, environment, materials and digital codes play a fundamental role. It promotes the designer as the creator of an openended system able to provide the rules for a variety of evolutionary forms to emerge, where final decisions are made by the resonance and collective intelligence of multiple agents, including codes, humans and machines, rather than a top-down unique design and final form. In your view, what kind of new collaborations could In yourview, what kind of new collaborations could augment their capacities to work with machines?

PFY: According to the philosopher Andy Clark, as human beins we are 'natural-born cyborss' The development of increasingly sophisticated disital tools and prostheses - from robotic fabrication to At - have been making us ever-more cyborg-like. In this posthumanistview, we we adh with former adapts to digitaltechnology.

Furthertore, whindustral robots as the revolutionary constrution platform in the digital era, the architectural profession is experiencing a paradigm shift from tradtional craft andindustral reproduction to cyborg craftsmanship techniques, combining human-toNew possibilities for collaboration, made possible by this robotic platform, challenge traditional design authorship and question authority whin the cycle of architectural toward de-professionalisation within the building industry emerges through the reciprocal feedback loop between cloud computation and production.

Shared knowledge and the fresh creativity liberated by the platform between robots and humans encourage the living environment. In this way, construction tools have and systems of fabrication can be customised or modified according to the intention of the user. Based technology introduces a highly adaptive prefabrication system, which is distinguished from the assembly system based on mechanical reproduction in the post-war period. With this upgrade of the construction system, triggered by customised reproduction, the feedback loop between tool and user will become highly differentiated. These differential feedbacks are inserted into the social production system, and a new relationship between architects, technology and the building industry would be established. How architects participate in digital technology adaptation thus becomes an essential question for the future. citizens to contribute to the constant building process in a kind of reversed adaptivity, too. Processes, interfaces on robotic platforms and Al, customised fabrication

Bibliography

Brynjolfsson E. and McAfee A., 2014, The Second Machine Age: Work, Progress, and

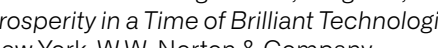

Clark A., 2003, Natural-Born Cyborgs: Minds, Technologies, and the Future of University Press.

Dubor, A., Izard, J.B., Cabay, E., Sollazzo, A. On-Site Robotics for Sustainable

Construction' in Willmann, J., Block, Hutter, M.. Byrne, K. and Schork, T. (eds.), Design 2018. Cham Switzerland Springer International, p.390-404.

Ilen MacArthur Foundation, 2012, Towards Business Rationale for an Accelerated Transition, Isle of Wight, Ellen MacArthur Foundation.

Gausa M., Markopoulou A. and Vivaldi J.,
D19. Black Ecologies, Barcelona. Actar Publishers.

Surowiecki J., 2005 (second edition),
The Wisdom of Crowds, New York, Anchor. Note

1. Circular design systems aim to prevent or
limit material and resource loss, and have the potential to minimise waste, using this

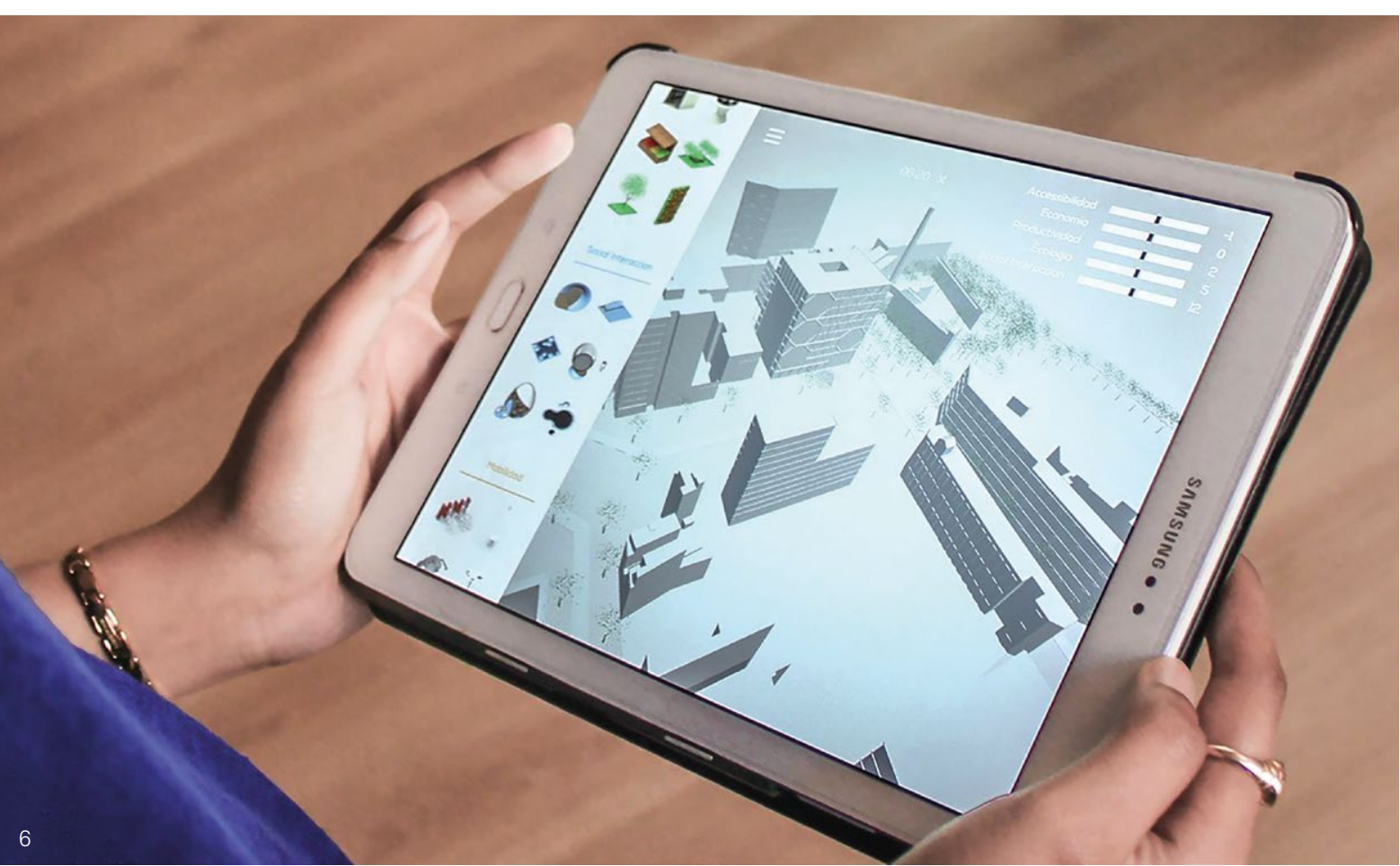




\section{Agency of Material Production Feedback in Architectural Practice} Tom Svilans

Centre for Information Technology and Architecture (CITA)

The Royal Danish Academy of Fine Arts

Jonas Runberger

White Arkitekter AB, Stockholm, Sweden

Kai Strehlke

Blumer Lehmann AG, Gossau, Switzerland

\section{Introduction}

With the introduction of automation, computation and large-scale engineered timber, contemporary timber design and fabrication have evolved from earlier craft-based traditions. Projects have grown in scale and complexity with this evolution, facilitating new forms of building and uses for timber in architecture and construction. The complex nature of wood - a live and organic material - presents challenges for fabricators and designers, however, due to its tendency to change form in response to environmental and inherent factors. This is especially felt in the development and construction of large-scale, freeform timber buildings, where the of large-scale, freeform timber buildings, where the performative demands of wood are much higher. Advances in computational work flows and machine real material behaviour as an input to both the contro systems involved in fabrication, as well as the early design stares and disital models of an architectural design stages and abricalion, as well as the early project. This presents apportus of an archicectural of -aware design-to-fabrication

aterial behaviour and performance in the design and fabrication of freeform timber stuctures is the fous of a parthership betwe an Innochain Ealy-Stage Researcher (ESR), Dsearch the computationt design team atWhite Arkitekter $A B$ - and Blumer tehmann $A G$ a specialing in the developmentand productractor complextim the development and production of from this collar projects. Here, we present a case study trim industry secorn Stockolm, Sweden, informed by two industry secondments with both industrial partners over The key point of focus is how research can be conducted within the contrasting settings of industrial timber fabrication and multidisciplinary architectural practice during live projects, allowing material performance and feedback to be integrated in both domains. A secondary focus is to show how research conducted in parallel industry environments (architectura practice and production) can broker expertise between these environments, as exemplified in the production feedback in early-stage design in architecture.

Integrating Material Performance

Using a multi-scalar approach, we consider different forms of feedback: feedback in design through computational models and augmented modelling tools; direct feedback in production through the integration of sensors and $3 \mathrm{D}$ scanning in the timber production process; and organisational feedback in the timber supply chain through the exploration of iterative integrated gluing and machining processes (Svilans int. 2019). Previous work hing processes (Svilans et multi-scar modelling for the design and fabrication of miliscar and other research collaborations (Svilans, et a., 2018). This case sudy howerer, focuses on the transference This case study, however, focuses on the transference of reserch results to inm indust in. The relevance or this is augmented by the large shit in architecture toward an in and methods of production. the 'diglal continuum' between design and construction (Kolarevic, 2003). This continuum has been extended beyond methods of production to encompass the design of materials and harnessing of their behaviours. Using digital simulation as an integrated component of the design-to-fabrication process has resulted in new material practices that

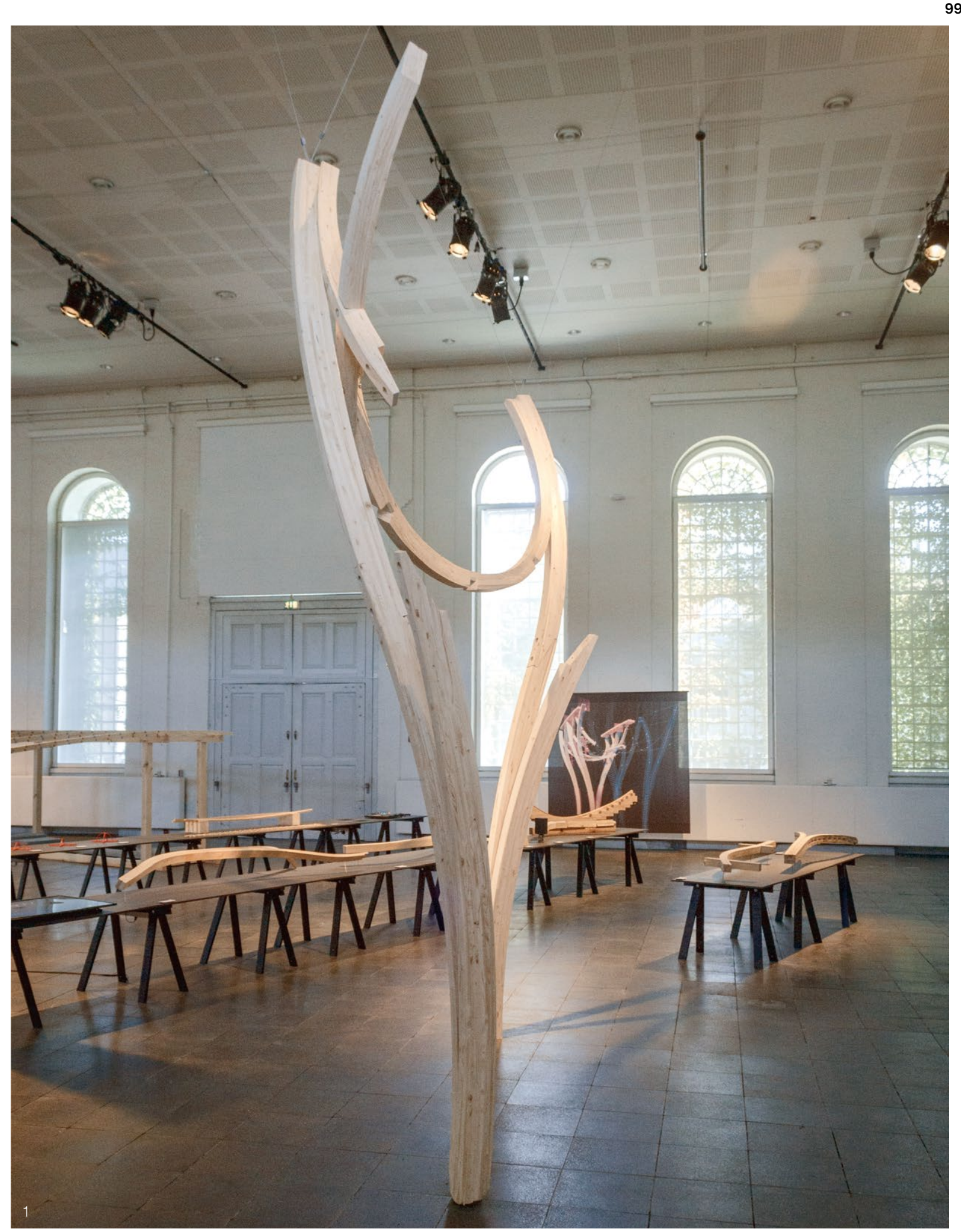

1. The physical mockup of a portion of Version 3 Building Design for a New Material Age'. 

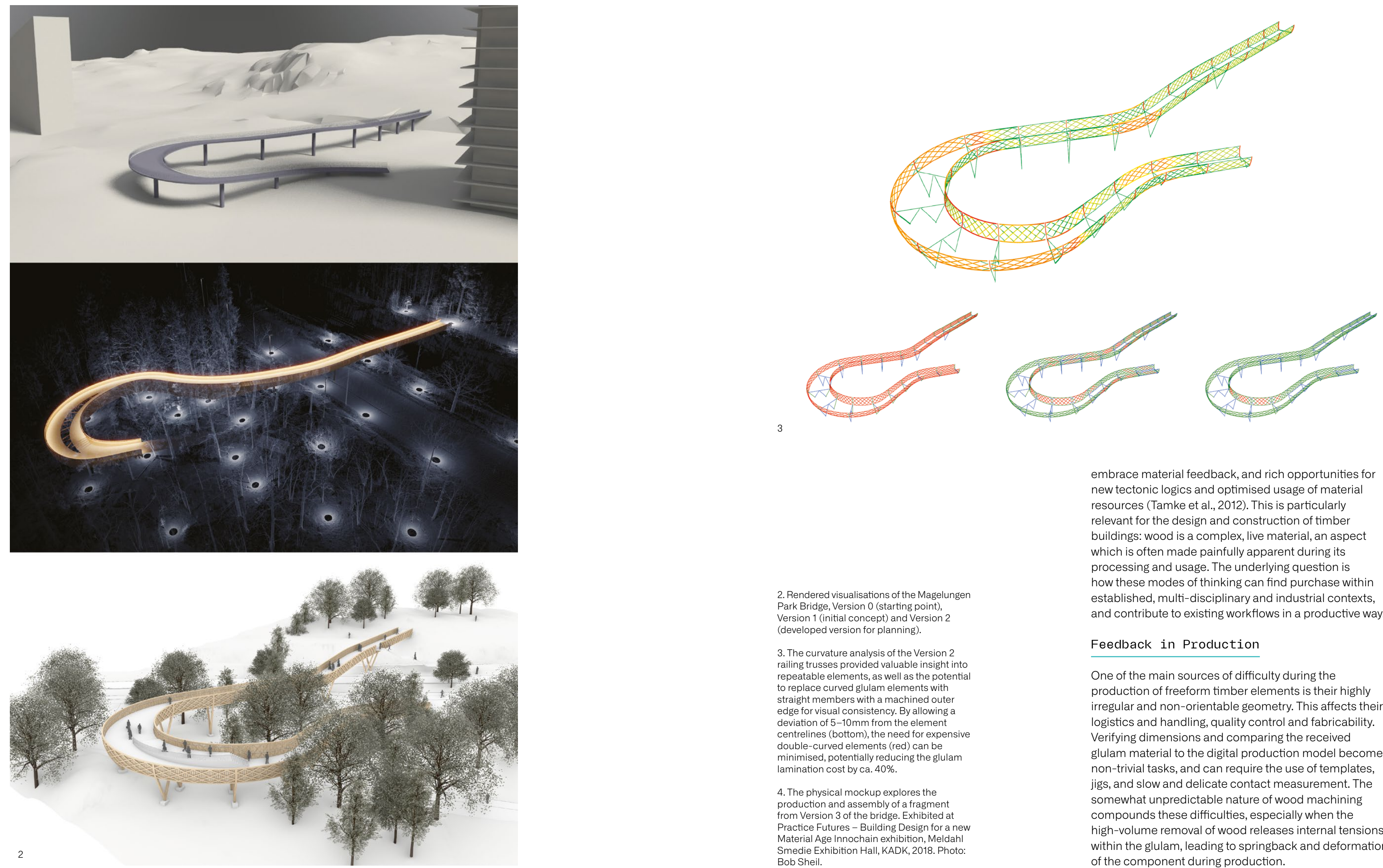

2. Rendered visualisations of the Magelungen Park Bridge, Version 0 (starting point), Version 1 (initial concept) and Version

3. The curvature analysis of the Version ralling trusses provided valuable insight into to replace curved glulam elements with straight members with a machined outer edge for visual consistency. By allowing a centrelines (bottom) the need for exponsive double-curved elements (red) can be minimised, potentially reducing the glulam

4. The physical mockup explores the production and assembly of a fragment Practice Futures - Buidge. Exhibited at Material Age Innochain exhibition, Meldahl Smedie Exhibition Hall, KADK, 2018. Photo: embrace material feedback, and rich opportunities for new tectonic logics and optimised usage of material resources (Tamke et al., 2012). This is particularly relevant for the design and construction of timber buildings: wood is a complex, live material an aspect which is often made painfully apparent during its processing and usage. The underlying question is how these modes of thinking can find purchese within established, multi-disciplinary and ind ustria contexts, and contribute to existing workflows

\section{Feedback in Production}

One of the main sources of difficulty during the production of freeform timber elements is their highly rregular and non-orientable geometry. This affects their ogistics and handling, quality control and fabricability. Verifying dimensions and comparing the received glulam material to the digital production model become on-trivial tasks, and can require the use of templates, jgs, and slow and delicate contact measurement. The somewhat unpredictable nature of wood machining compounds these difticulties, especially when the high-volume removal of wood releases internal tensions within the glulam, leading to springback and deformatio 
The extreme cost of downtime made choreographing the experiments around active production schedules a challenge. The research strategy, therefore, becam one of 'shadowing the production through parallel development of the scanning experiments, with short and focused testing periods at key points within the production schedule. If providing immediate and positive impact on production, they were implemented, and, if not, they could be easily rolled back. This allowe the continuous parallel experimental research to be tested within the scope of production, allowing flexible development with very clear performance criteria while avoiding the need for a dedicated production setup.

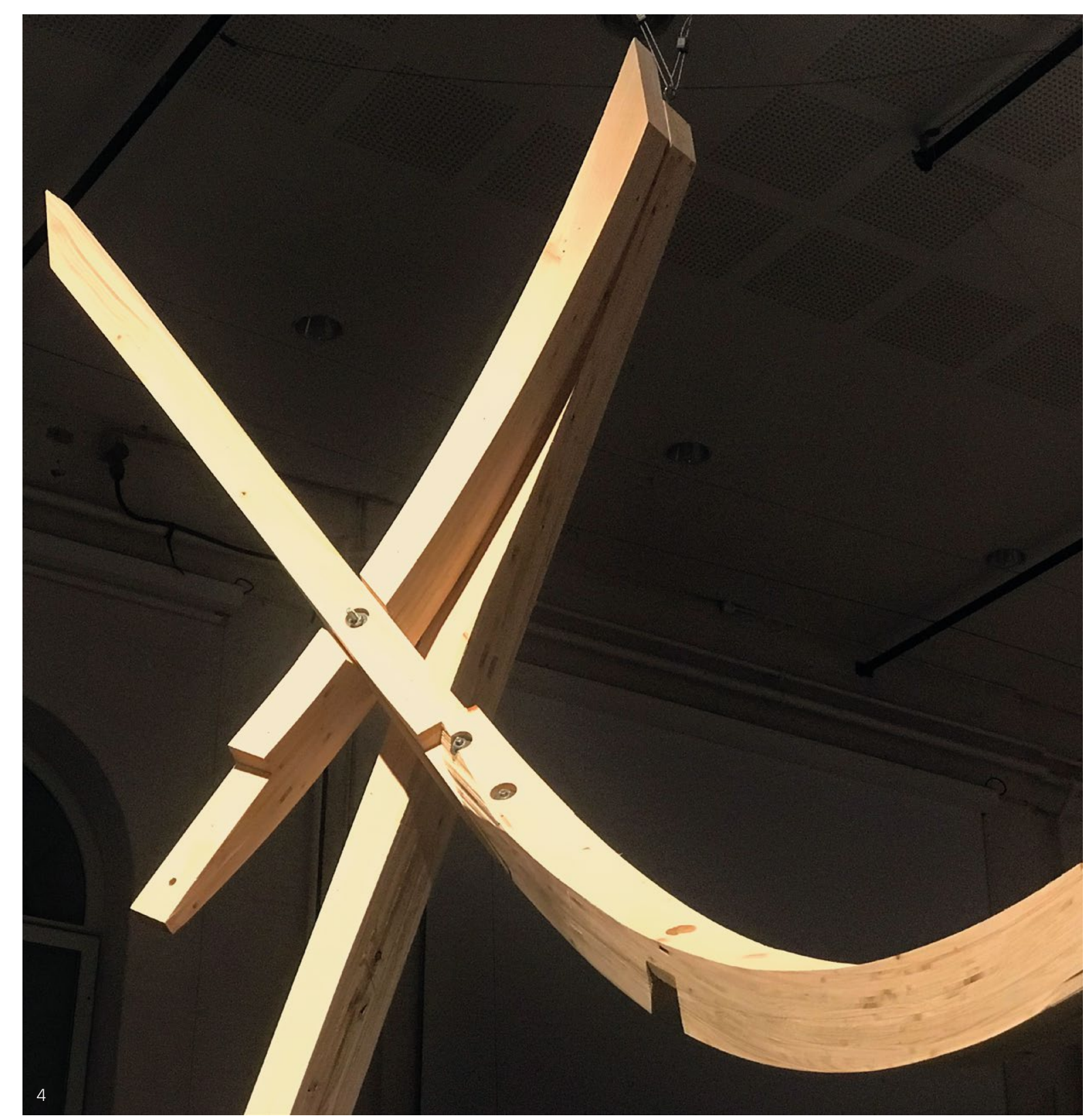

These experiments were the first steps toward a tighter Thegration of material behaviour and the development of a more fluid continuum between model and materia in an industrial timber context. This has two main implications, the first being the development of an 'adaptive fabrication model', where dimensional changes in material can be addressed and responded to during linear production. Production often runs in a kind of 'fire and forget' mode: material is ordered, the production data is generated, and then the processing is blindly trusted to the performance of the machine and the accuracy of the material. 'Eyes' that link the ongoing processing with the digital production model in real-time or at discrete intervals minimise the risk and unpredictability involved. Responding to dynamic material behaviours would reduce risk and improve the overall quality of machining, as well as lessen the time spent estimating, checking and cautiously moving forward. The second opportunity is that of an 'encoded experience' through the gathering of sensor data across projects and over longer periods of time. This would allow both the fabricator and designer to extract insights through statistical means about the types of input geometry, material and production strategies, and how they impact on performance. For producers, this would provide a much more accurate assessment of risk and expected quality.

For designers, it would serve as a sounding board for testing design ideas, as well as providing a good base of principles for freeform timber design. The geometry and curvature of freeform glulam elements have a direct

impact on their cost, structural performance and visual appearance. The composition of elements and the way in which they are processed affects their form stability and end quality. Extracting these principles and insights at an early design stage, therefore, becomes a priority for the design and construction of smarter and more efficient timber structures.

Material Performance in Early-Stage Design in Architectural Practice

The Dsearch team members at White Arkitekter perform various different roles: from back-end method perform various a ment The ESR secto support and design lead on projects. inition the Mage the Magelungen Park Project in Stockholm, featuring Theaping and five differentrosidential developments. The bridge will provide pedestrian and bicycle access between two separate forested areas, biliging overa road, a set of commuter-train tracks, and pedestrian/ bikng paths, with a height difference of approximately $10 \mathrm{~m}$ from start to end, requiring it to be extended into nearby wooded grove in order to achieve a maximum intilly planned as a standard bridge in steel or concrete, . The research/practice collaboration enabled an mproved design outcome, and gradually introduced client and stakeholders to this more advanced structure of higher architectural merit and better use of resources, while maintaining cost efficiency. The methods developed by the ESR could be directly applied in the well-established computational workflows of Dsearch (Runberger et al., 2015), facilitating the direct transfer of research into practice. The role of the ESR here shifted from a 'shadowing' approach to an active collaborative design role, followed by a divergence into two parallel development trajectories, resulting in Versions 2 and 3. The planning process for the bridge is still ongoing at the time of writing, with forthcoming steps to be based on the research outcome presented below.

Version 0 can be seen as the starting point and a point of reference for the more refined versions. The curving form is dependent on the need to extend the bridge to allow the maximum $5 \%$ incline.

In Version 1 the researcher proposed a first conceptual principle using curved glulam beams as well as timber panels. At this stage, the different structural needs due to differences in spans of approximately $30 \mathrm{~m}$ across the road and railway, versus $9 \mathrm{~m}$ over land in the grove - were identified but not resolved. The structure of the bridge was configured as repeated $\mathrm{V}$-formed columns carrying horizontal glulam elements that formed the edges of the bridge, with timber elements between the edgebeams to create the floor. The longer spans were provided by a shift of structure, from very short spans distributing the load to a truss based on a central beam across the road.

Version 2 was repositioned onsite according to 3D-scanned data, avoiding damage to high-value oak trees. The railing became structural, using trusses that minimised the depth across the highest point over the railway and allowing the minimum free height to be achieved without further extending the bridge in plan. In the initial teration, the truses of the bridge riling were clad with externa vertical panels. In the second wertion, whe structure was inverted, with an execrallycurved truss and interna chadding, wh an externallyced to be properly covered and a simpler sood for the in be of the riling to discour age climbing The for he to a portial solution whe beans of lesser curvatue cou pon ould be replaced with straight beams with a machined 73 inger As a result, he inthal estimate of 61 straight 73 single-curved and 654 double-curved elements could be adapted to 268, 500 and 20 respectively, with a potential glulam lamination cost saving of around $40 \%$. Version 3 put the focus on utilising more of the structural potential of curved glulam elements by 
bridge, and testing the design-to-fabrication workflow and toolkit developed in both secondments. The goal here was to arrive at a physical mockup that would demonstrate the feasibility of the integrated approach, linking material feedback and production concerns to the design modelling and overall bridge scheme of the Magelungen Bridge. As such, the design involved more use of single- and double-curved glulam elements that were modelled and rationalised using the design

modelling tools. For example, the overall geometry was constrained so that most blanks could be single-curved with minimal cutting of double-curved surfaces, and to maximise the thickness of individual lamellae to make production easier. The direct feedback techniques developed at Blumer Lehmann were applied to locate and verify the material during machining, facilitating the processing of the freeform elements. The final physical prototype was fabricated by the ESR in an environmen that mimicked the production context at Blumer Lehmann but on a smaller scale: an industrial five-axis wood processing centre using standard G-code.

Conclusion

The chronology of the secondments progressed, in a way, in reverse: starting with a 'shadowing' role in production and ending with a collaborative-design role in architectural practice. This order was intentional and facilitated a valuable transfer of expertise from production to design, providing the ESR a unique role when entering the practice context. The value of this transfer, as well as computational toolkits relating to material performance - from fabrication and production to early-stage design - could be identified in several ways in the given example. The pos the design at a conceptual stage while being directly the design at a concestrints of production and mectly performance aspects of bent, lominated wood was crucial to the project The iterative development allowed the shift of scope in the project from a standard bridge to an advanced slulam timber bridge, in a way that allowed stakeholders and decision-makers to initialy allowed stakendders accept and $40 \%$ cost risuction for lut production furthersupports this decision. These aspects can be seen as ways to control isk whe faciltating Imagnative movation in practice (Marbe, 2010). The use of mockups remains particularly important in freetorm timber structures and, although much can be simulated and predicted, the final pertormance depends on many factors that simply have to be explored through making. verification through physical prototyping is, therefore necessary at key points within this iterative design process. The preliminary outcome of the conceptual design and analysis stages will depend on the further use of prototypes and mockups.
Acknowledgements

project was undertaken at CITA, KADK, as part of the Innochain Early Training
Network. The Doctoral Thesis of the ESR Network. The Doctoral Thesis of the ESR Thomsen and Martin Tamke (CITA) with additional industry supervision by Kai Strehlke (Blumer Lehmann), Jonas Antemann (Design-to-Production). Thanks to the staff and faculty at Aarhus Architecture School for their contribution toward the All images copyright Tom Svilans, Blumer
Lehmann AG or White Arkitekter AB, unless otherwise s

Bibliography

Kolarevic, B., 2003, 'Introduction' in Digital Age: Design and Manufacturing Didcot, Taylor \& Francis, p.3-10.

Marble, S., 2010, 'Imagining Risk' in Deamer, P. Future: Recasting taborin Architecturess, Princeton, NJ, Princeton Architectural Press,

Runberger, J. and Magnusson, F., 2015, "Harnessing the Informal Processes around the Computational Design Model' in
Thomsen, M. Tamke, M. Gengnagel Faircloth, B. and Scheurer F. (eds.), Modelling Behaviour, New York, Springer, p.329-339.

Tamke, M., Hernández, E.L., Deleuran, A.H., Gengnagel, C., Burry, M. and Thomsen, M.R.,
2012, A N New Material Practice: Integrating 2012, 'A New Material Practice: Integrating
Design and Material Behavior', in Symposium on Simulation for Architecture and Urban Design '12, p.1-9. http:///d.acm.org/citation
cfm?id=2339456 (accessed 8 February cfm?id=2339.
2020).

Svilans, T., Poinet, P., Tamke, M. and
Thomsen, M. R. 2018, 'A Multi-Scala Thomsen, M.R., 2018, 'A Multi-Scalar Approach for the Modelling and Fabrication Structures' in Humanizing Digital Reality New York, Springer, p.247-257.

Svilans, T., Tamke, M., Thomsen, M.R., 2019, 'New Workflows for Digital Timber' in Bianconi, F. and Filippucci, M. (eds.), Digita Reodresentation in Architectural Design

New York, Springer, p.93-134.

\& 6. Production of the physical mockup for Version 3 integrated the glulam modelling . 


\section{Acoustic Wall:}

Computational and Robotic

Design Integration of

Four Primary Generators

Isak Worre Foged

Aalborg University

Anke Pasold

Copenhagen School of Design and Technology

Mads Brath Jensen

Aalborg University

\section{Introduction}

This study pursues the idea and potential of designsteered convergence processes, using the assembly of a limestone acoustic wall as a 'vehicle' for exploring the integration of computational search methods, pattern expressions, acoustic simulation and collaborative robotics in design and making processes that are then tested and compared in terms of their acoustic performances. The work was initiated from visual and acoustic observations at Piazza San Marco in Venice Italy, and the Antalya-Demre Limestone Quarry in Turkey. Venice has an impressive display of articulated limestone, where the combination of colour nuances, geometries, patterns and stone erosion provide the unique characteristics that attract thousands of people unique characteristics that attract thousands of people to the city every year (fig.1). While it is reputedly the most visited square in the world, Piazza San Marco is also posited to be one of the most quiet, due to the lack of traffic and the acoustic properties of the stone used in the buildings (Horowitz, 2013). In the case of Turkey, upon invitation to the Istanbul Design Biennale, we we brought into direct contact with the Antalya-Demre Limestone Quarry, which provided a unique opportunity to study, explore and identify integrated design processes, including acoustics, limestone, design computation,
processes.

\section{Background}

The objective to combine and search design convergence through many aspects and underlying parameters (fig.2), is a common condition in design
processes (Dorst and Cross, 2001; Lawson, 2006).

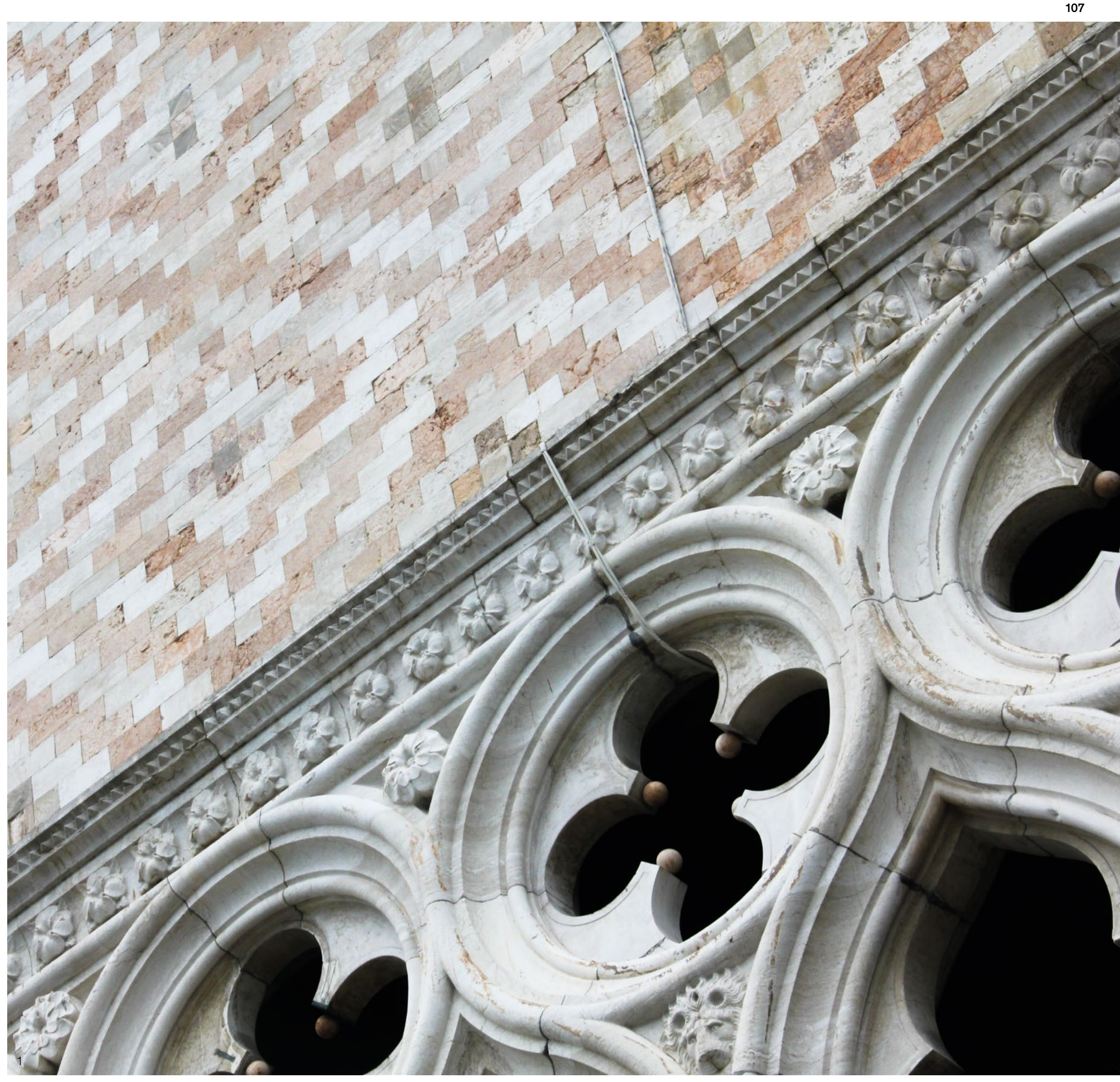




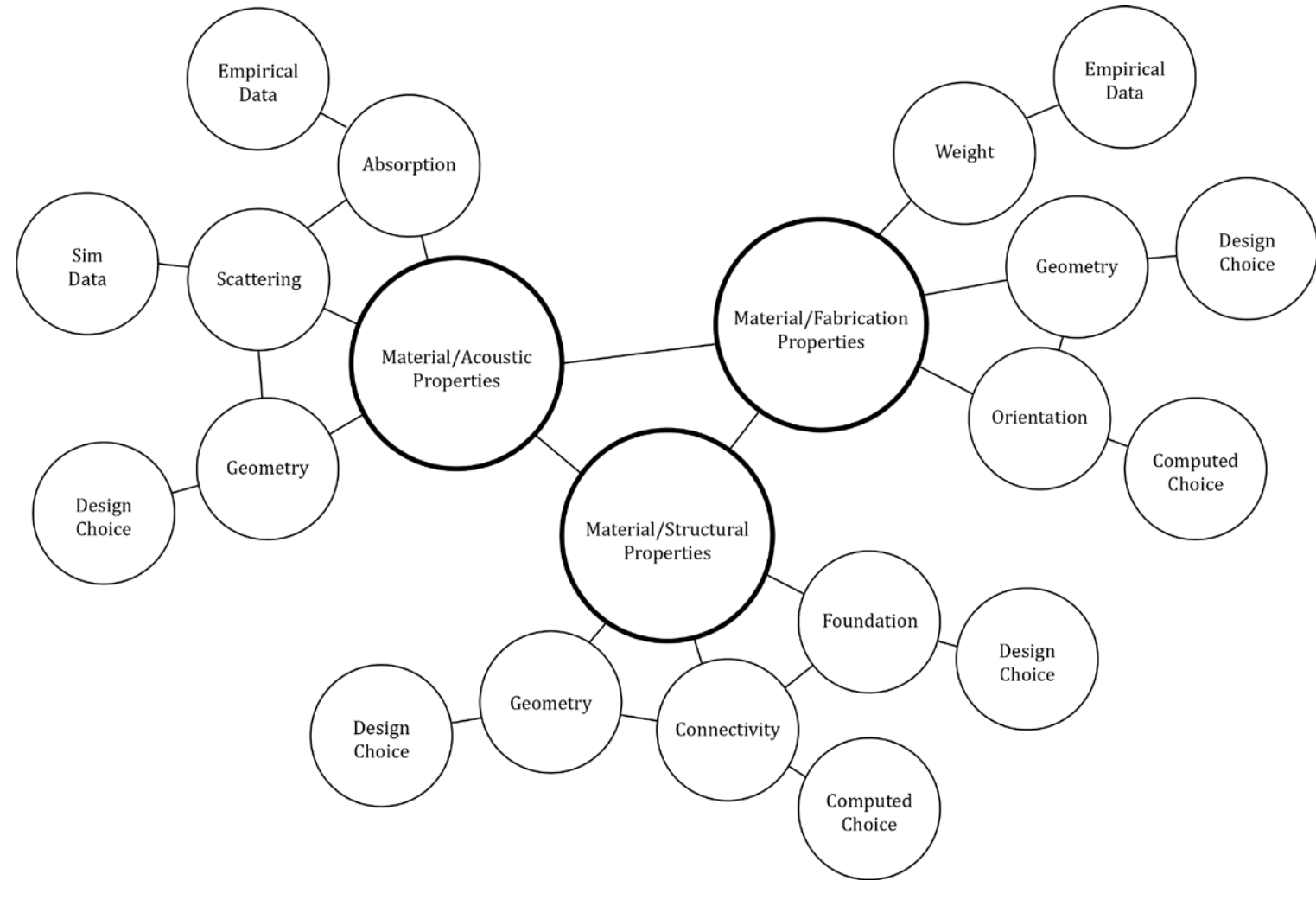

Such design conjectures, which aim to form 'wholeness' (Alexander, 2009) may, however, be based on simplified integration if not supported by specific design models which through distinct primary generators, which strategically integrate prioritised aspects (Darke, 1979; Foged, 2018). Consequently, this project attempted to explore collaborative robotics of complex assembled structures, where material properties, acoustics, assembly stability and human-machine interaction implemented into one explon By considering the human directly in the

computational design and robotic fabication loop, the project's methods and techniques attempt to support the creative and craft capacities of collaborative processes between systems and humans. Four primary processes between systems and humans. Four primary aspects - optics, acoustics, robolics ans statics - were therefore, in ferwoven in to the ealy design processes to seamlessly connectrobotic controwith human cratt in the computational design model and construction processes, as a way to build noverstuch learning by making.

to collaborative robotics in

architecture stem from a growing awareness of humans influence on design iterations during robotic making, the intuition and cognition of the operator augmenting the skills of the robot, just as the robot augments those of the designer' (Johns et al., 2014). It becomes necessary to categorise the components of these exercises within related in the field, and to their larger consequences within the architectural discipline. In this chapter, we present a number of approaches to robotic design/ fabrication exercises that deal with information, interactivity and material dynamics.

The project's aim in relation to fabrication, was to The project's aim, in relation to fabrication, was let sime relation between robot and maker, racher than applying complex high-tech visul projections or augmented agents - robot and person- relate through intuilive logics of comm ication, Just as technologies are limited to what is needed. In respect to the design model and its logics, the integration of multiple aspects to inform the brick bo the wall assembly challenges conventional brick bonds. The proposed visual and acoustic-driven composition of geometrically simple bricks can therefor serve as inspiration and be directly adopted by the creative and manufacturing industries working with masonry structures. a number of directions and motivations which can be really technologies. With his approach, the two making

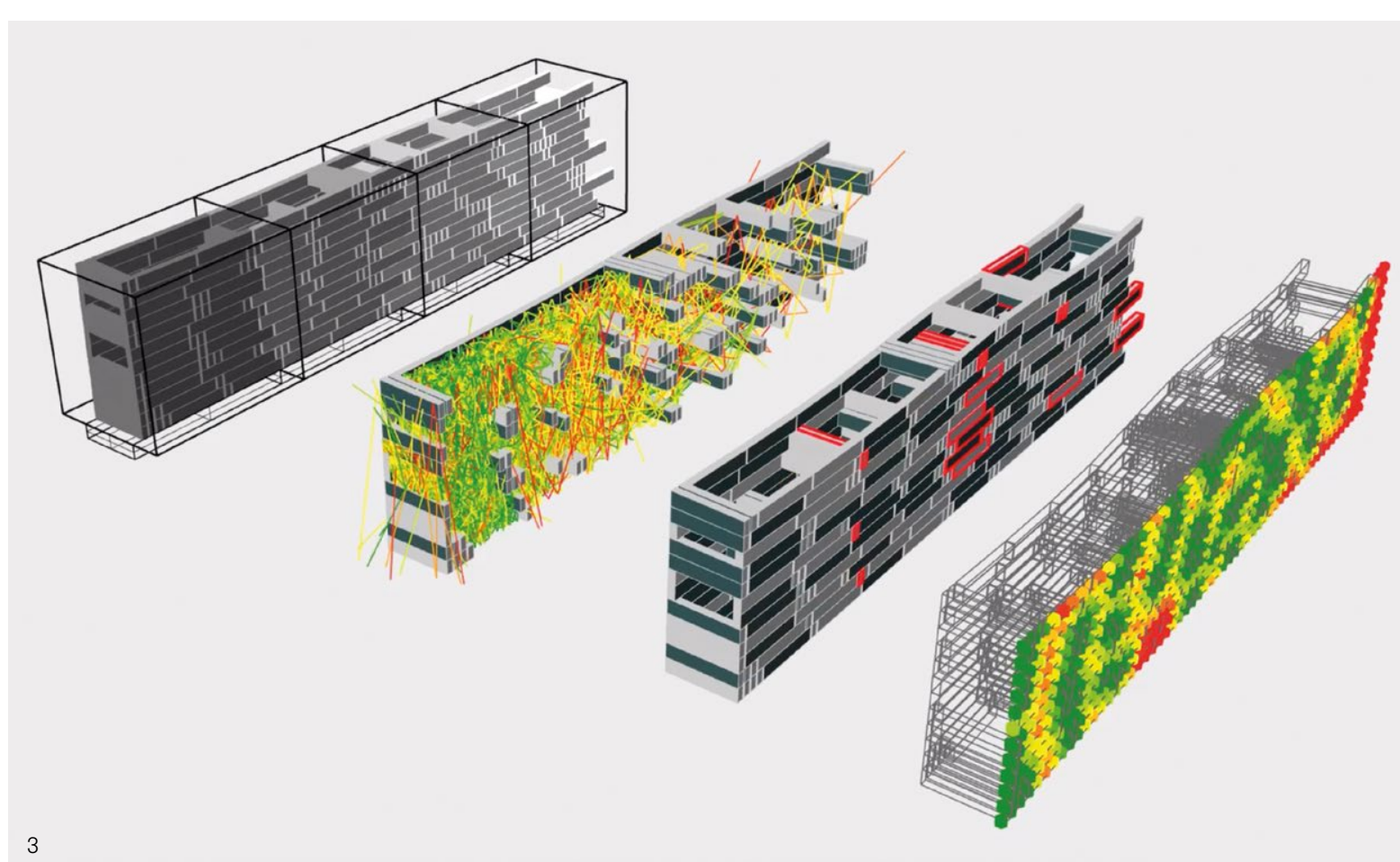

The studies in acoustics integration applied the Pachyderm acoustic simulation software into the parametric model, and describe each material element through geometric, material absorption and scattering coefficients. This enables a design search procedure to compute and steer the sound energy that is distributed through each individual double-sided wall and brickbond iteration (fig 3).

Using this approach, we sought to develop and investigate the combining of multiple primary performa mode. The model includes craft methods mesign properties, local materia sourcing acoustic simulation, fabrication constraints, fabrication control and communicotion with an farication control and The disication with an The dist com demonstrator uhlising limestone bricks measuring $30 \mathrm{~m} \times 60 \mathrm{~mm} \times 300 \mathrm{~mm}$, which were assembled into a sound-distributing double-layered wall with cross binders. Each limestone bick consisted of one rough side and five smooth sides, which acted as material variables in the search for a limestone wall composition that satisfied project-specific acoustic, structural and
expressive characteristics.
2. Diagram of primary integrated aspect and sub-parameters of the computational
design model for steered

3. Computational Design Model, including four primary design generators. From left a) robot fabrication limitations; b) hybricsupport assessment; d) brick bond 
110

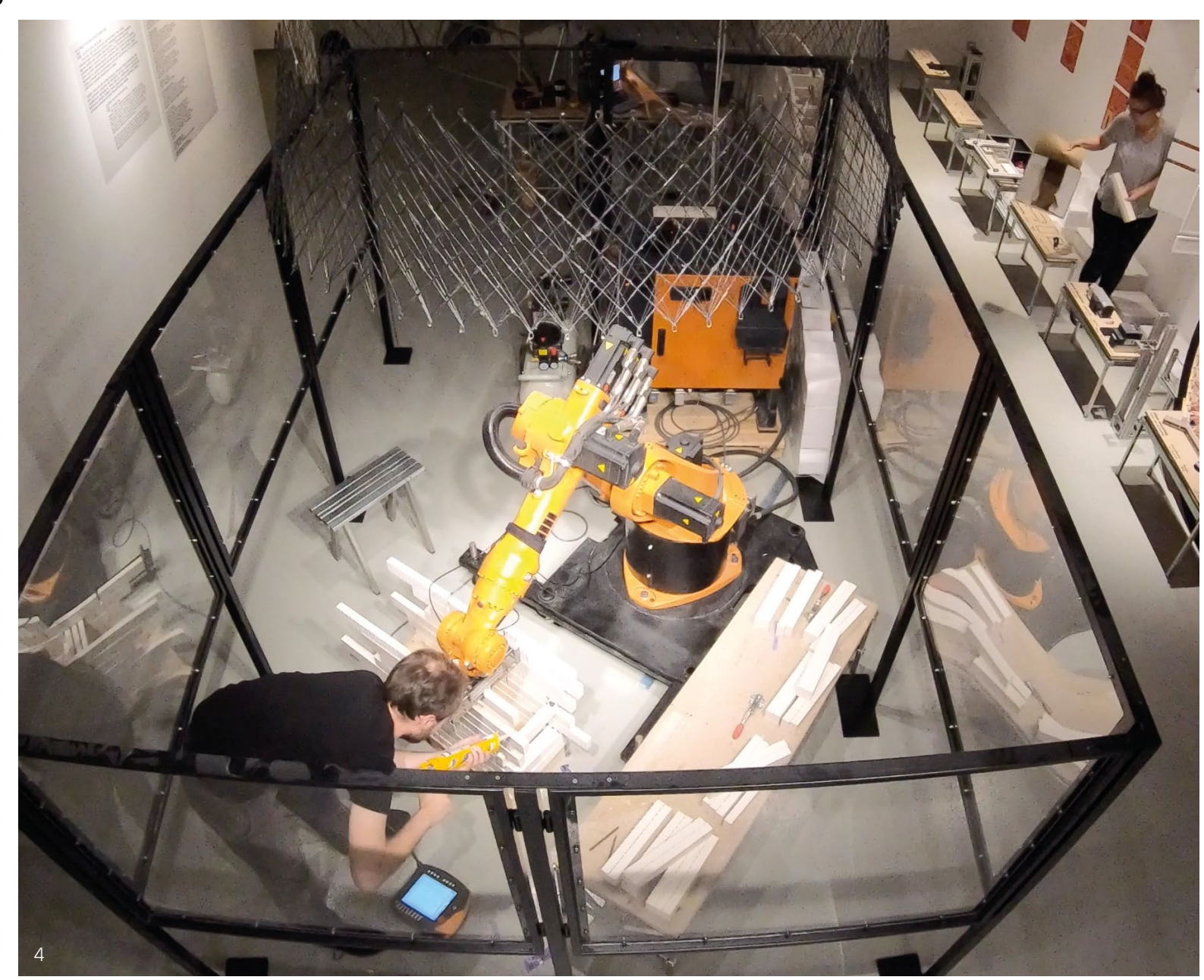

The studies present the design model for convergence search, enabled by communication between robot and human collaborator in the makin process. A brief description of the computational aspects will be presented here, elaborating on material practice, acoustic simulation, dynamic assessment practice, ilities of the bricks as they are layered by the of insta acoustic simulion, dy amic assess by the robotic making procedure, and assess

Methods

The studies were conducted using three methods/ The studies were conducted using three methods/ with a Computational Design Model; a Collaborative Robotic Model for assembly; and Acoustic Field Robotic Model for assembly, and Acoustic Field

\section{Computational Design Model}

The experimental research was based on the Rhino/ Grasshopper computational framework by McNeel Inc
where a series of plugins and bespoke Python-coded components were integrated into a complete integrative design exploration model These included Kuka I PRC for robotic control devel. The by holuded Kuka I PRC Pachyderm Acoustics, developed by Arthurvan der Harten: Goat developed by Rechenraum; and de Hartearch conditions and structural instability tests developed by the authors.

\section{Collaborative Robotic Model} The robotic arm used for positioning and gesturing
is a Kuka KR2O-3, with a modified SMC MLH2-2OD pneumatic gripper mounted as end-effector. These technologles were used to ilustrate that readily accessible tools and techniques can be applied, following this research. A gesturing system, using a Leap Motion sensor, was constructed and used during testing, and a bypass communication system - in the form of a simple push-button - was implemented for communication between robot and human during the making of the brick wall demonstrator. is a Kuka KR2O-3, with a modified SMC MLH2-20D and easily converted into industry-oriented projects

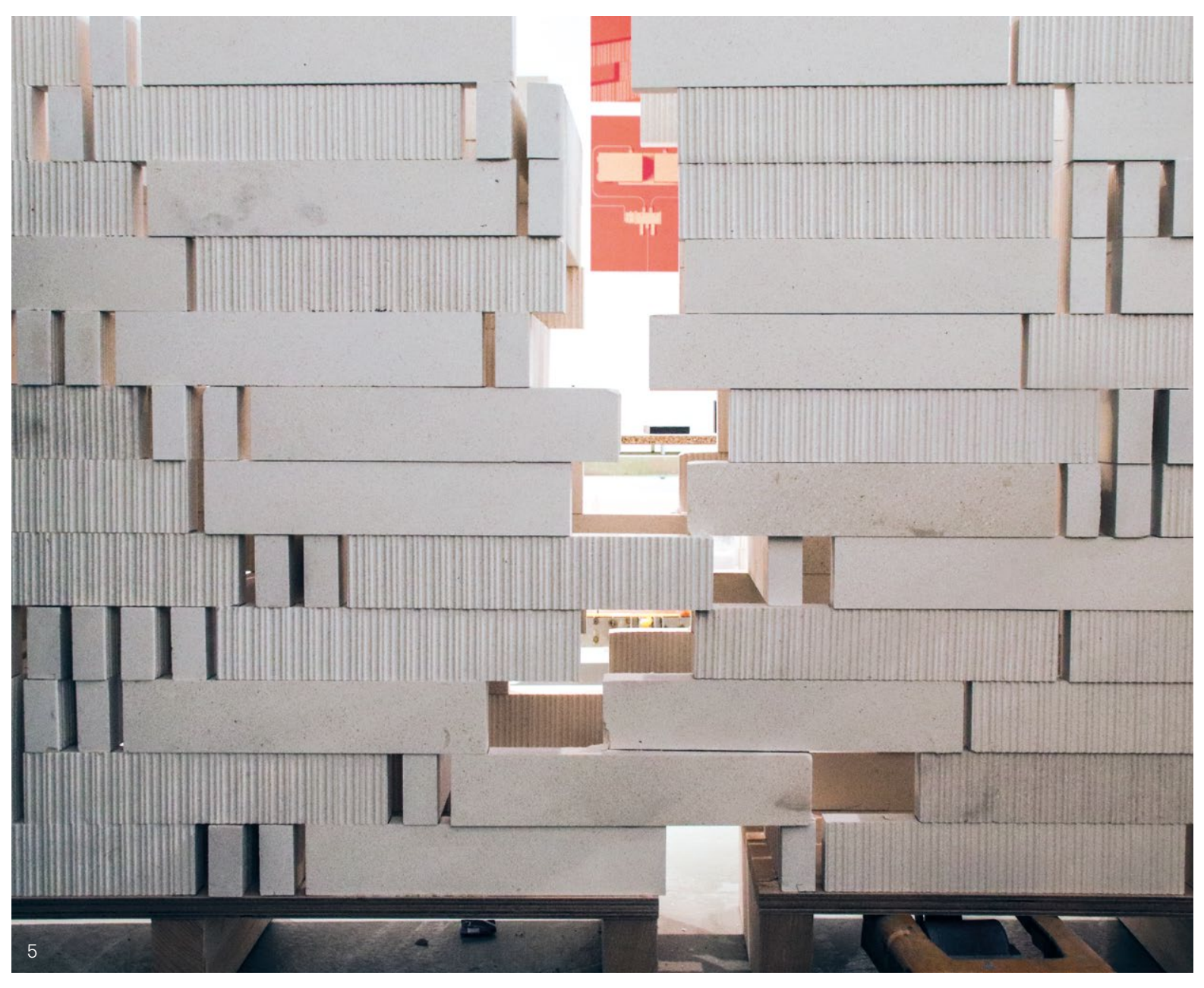

Acoustic Field Measurements

An initial set of acoustic field measurements were taken at Piazza San Marco, using a Class A condenser microphone (i365) with calibrated sound meter software on an iPhone 8 to determine whether the square could be registered as acoustically 'auiet' Sound pressure measurements were conducted at three different ime of day across the square, to cover the relative ines Following spe and account forvariation in occupancy. Istanbul Design Biennale, acoustic measurements con conducted to measure the loss of sound energy through we cavily between the two sides or the double layered wall, and hus to understand the sound transfer properties of the cavily based on material-geometric compositions. Measurements were taken using a measurement microphone and RØDETest FuzzMeasure software for sound energy analysis. process with limestone brick bonds composed through a design integration
process at the Istanbul Design Biennale 2018. Photos
Bicer Saner.

( human composed during the design integration
process at the Istanbul Design Biennale 


\section{Design Experimentation}

The intent behind the design experimentation was to explore how a computational design system and collaborative robot-based construction methods could create a novel double-layered acoustic-influenced limestone masonry wall. These design studies aimed to identify and propose ways for more complex assemblies to be defined and designed than single-layered mason walls. The computational design system's variables include a global form definition and a series of local conditions, where the composition weaves together surface and binder bricks, while maintaining stabill across the assembly (also during construction), increasing the sound transfer through the cavity, and modifying the position of the surface and binder bricks, and the orientation of both rough and smooth sides (fig.3). Furthermore, a series of preliminary physic design tests were conducted with scaled bricks to test how the computational, robotic and construction setup could scale to other dimensions, and allowing for an understanding of the system's adaptability to subjective design input.

The study also explored the role of the human co-worker by constructing methods allowing for communication between human and robot during the fabrication process. These experiments also therefore explored the division of labour between human and robot related to the brick stacking and gluing processes. As previously mentioned, hand gestures were used as a method for communication between human and robot a method that, despite its simple setup (counting of fingers and orientation of the hand), required addition a verification to ensure that a recorded hand gesture was indeed the one intended by the human collaborator.
This process was necessary for removing the uncertainty involved in delisering the correct command to the robot, although it also protracted the collaborative fabrication process. The verications were provided by the robot Arduino Uno board.

\section{Results and Discussion}

The computational design model, based on an ecology of off-the-shelf and bespoke Rhino Grasshopper components, with a two-phase stochastic and deterministic search procedure, was partly able to bring together the primary aspects integrated in this study. However, it was unable to operate or to respond with novel solutions when it encountered undefined conditions or unforeseen uncertainties during a design solution search. One study-specific example of this scenario is the design model positioning of the acoustic source and receiver as variables, which were steered by human intervention and presented significant performance gains that the defined search procedure did not. Hence, when the design model remains open to human steering interventions, possibilities for more novel solutions can be sought, whereas framed searches lead to closed-loop explorations, where human steering is limited to between computational search processes. For this reason, the study indicates that even integrated and advanced computational search processes in design need further abilities for steering in open design processes.

With respect to the collaborative robotic making process, guidance and in-automation steering was provided through simple hand-gesturing. This method, however, was quickly abandoned during the making of the demonstrator, in favour of the simpler buttonactuation command communication. One reason for this appeared to be an insecurty around whether the robot had understood a human gesture or not, and whether the correct robot action would be executed. Lack of confirmation cues between robot and human added unintended uncertainties. These uncertainties slowed down the making process significantly and ofte influenced the human co-maker to adjust their communication methods.

The study also shows that incorporating robotic fabrication within an undetermined and explorative design process requires a human-robot-material setup where the human co-designer can interact and interven during the fabrication process and make necessary changes when needed. For example, this could be due to the unaccounted-for dimensional variation of the material elements or, of more interest to this specific study, as a response to a new design intent arising from the human-robot fabrication process itself. Intervenin in the robot-driven making process will require a shift away from the predetermined file-to-factory approach and toward new design methods, supporting computational design models that can dynamically respond to design alterations made during fabrication. The specific design system studies and collaborative robotic making processes described here suggest the need for advancing human-system-robot interfaces. If they are to become integrated agile systems, which operate with and for human creativity and fabrication, their novelty lies in the intelligent interplay between human and system.

\section{Bibliography}

Andersen, R.S., Madsen, O., Moeslund, T.B. and Amor, H.B., 2016, 'Projecting Robot RO-MAN 2016: Proceedings of the 25 IEEE International Symposium on Robot and Human Interactive Communication, New York. https://doi.org/10.1109/

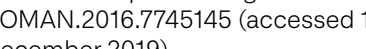

Alexander, C., 2009, 'Harmony-Seeking Computations: A science of Non-Classical Evolution of the Larger Whole' in International Journal of Unconventional

Darke, J., 1979, 'The Primary Generator and the Design Process' in Design Studies,

Dorst, K. and Cross, N., 2001, 'Creativity in the Design Process: Co-evolution of problem-solution' in Design Studies,

Foged, I., 2018, 'Integrated Design Processes by Sequential Primary Generators' in Journal of Problem-Based Learning in
Higher Education, Vol. 6, No. 1, p.66-87.

Horowitz, S.S., 2013, The Universal Sense. How Hearing Shapes the Mind, London,

Johns, R.L., Kilian, A. and Foley, N., 2014, Design Approaches through Augmented McGee, W. and de Leon, M.P. (eds.). Robotic Fabrication in Architecture, Art and Design 2014, New York, Springer, p. 319-332.

Lawson, B., 2006, How Designers Think: The Design Process Demys

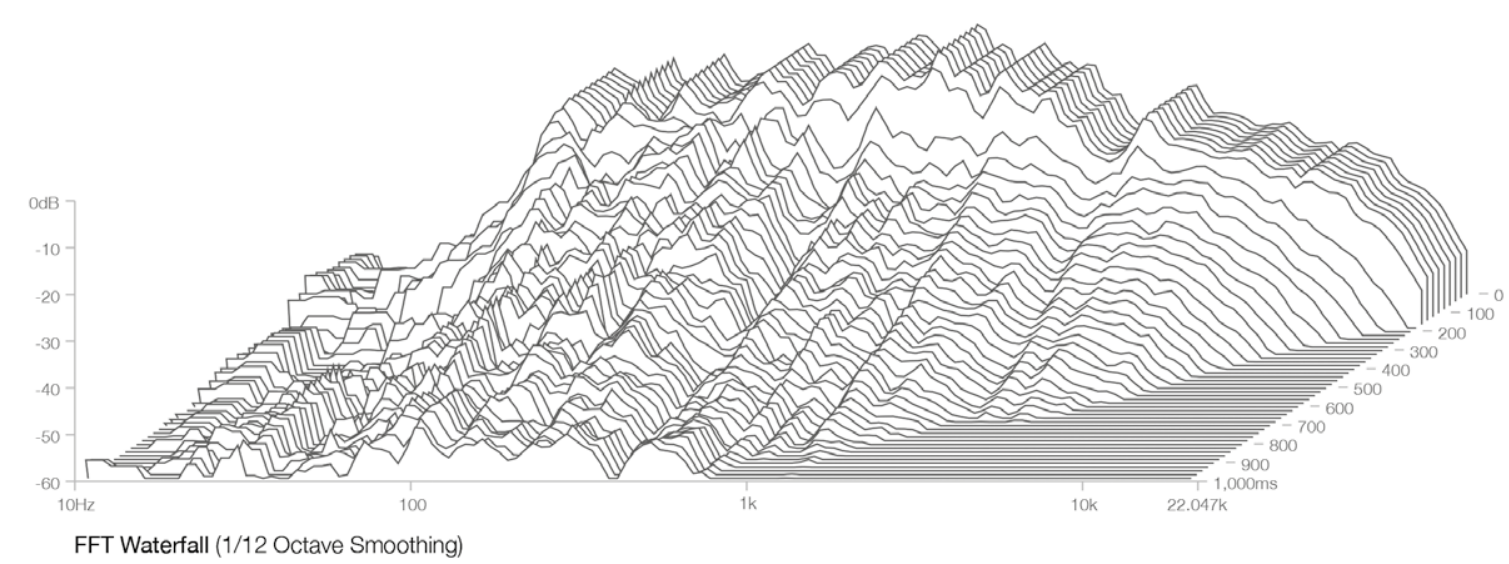

6. Fast Fourier Transformation graph from IR measurements of the demonstrator, showing the sound-energy distribution through the wall assembly. Sound energy maintained and transferred at low requencies through the structure. Graph by the authors. 
Coreless Filament Winding: From Academia to Practice

James Solly and Jan Knippers

Institute of Building Structures and Structural Design (ITKE), University of Stuttgart Moritz Dörstelman

FibR GmbH

\section{Introduction}

This paper presents the case study of Coreless Filament Winding, a digital additive manufacturing technology for architecture that is evolving from the world of academia into a commercial industrial environment. This example is relevant within the Innochain context, as it exemplifie a soft transition whereby links between academic institution and a startup fabrication company have been maintained for mutual benefit.

The Coreless Filament Winding Process

Coreless Filament Winding (CFW) is a fabrication method for the creation (FRP) parts. Since 2011, it has been under active development by the Institute for Computation development by the Institute for Computational Des

and Construction (ICD) and the Institute of Building

Structures and of Stuttgart. Detalls of the process were first published by researchers in relation to the ICD/ITKE Research Pavilon 2012 (Schuinn tal, 2013). CFW may be regarded as a vas Winding process where continuous fibre bundles are arily used in istent materia properties while placing material at high speed. As oullined by Schwn, the use of a core-based method has limitations for the production of architectural composite parts. Buildings often require many unique parts that require a range of unique cores. CFW was conceived to address the waste resulting from the large number of cores and the issues associated with the demoulding of core-wound parts. It involves the

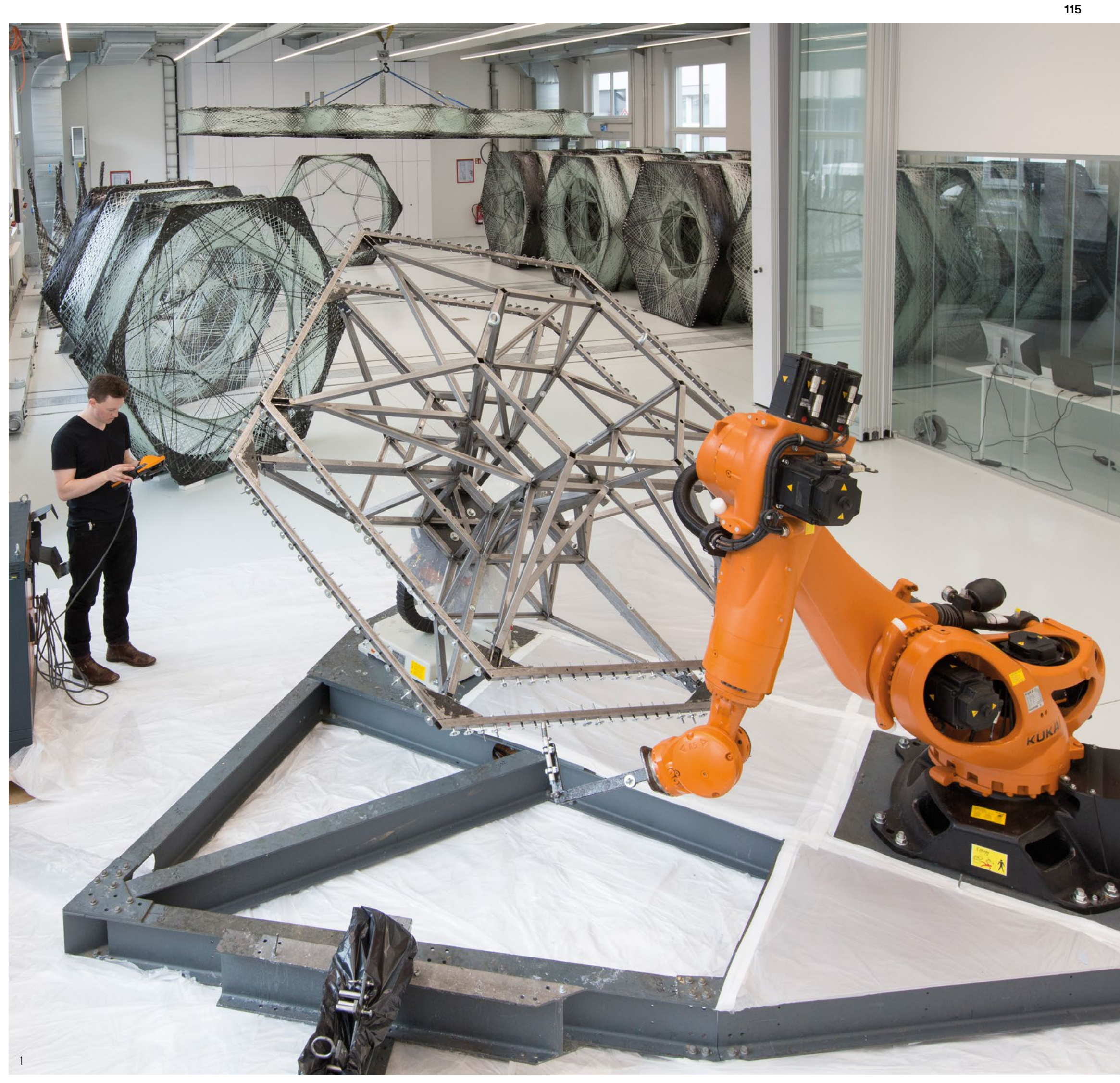




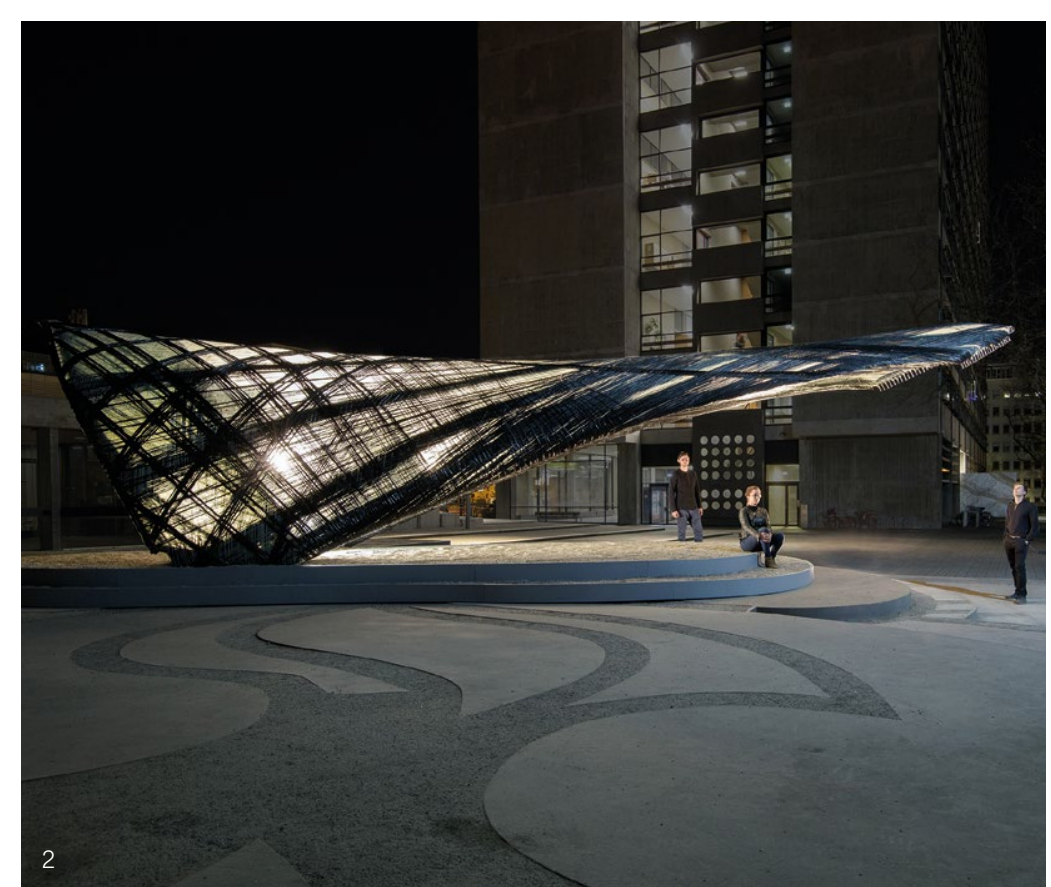

2. Research Pavilion 2016/2017,
O ICD/ITKE

3. Elytra Filament Pavilion under

Construction, O NAARO.

4. Slab component, 'Resistant Filigrees'

by Jorge Christie.

wrapping of resin-impregnated fibre bundles around spatially-located discrete winding pins. Fibres passing between these pins are sequenced to cross over one another, building up a geometry that emerges from their interaction. As this geometry is a result of both pin location and winding sequence, a single arrangement of pins enables several forms to be created through different sequences. The pins are typically mounted onto skeletal frameworks that can be demoulded and reused following curing (the setting/hardening of the resin) of the FRP component In some projects, such as the of the FRP component. In some projects, such as the ICD/ ITKE Research Pavilis adjustment in order to further increase the range of adjustment in order to further increase the range of The simplicity of the placement method - robotically winding wet fibres around pins - enables high-speed winding wet fibres around plins - enables high-speed materal lay-down addive mant to the material to harden during fabrication. In CFW, the machine-dependent fabrication stage operates time-critical pathway.

\section{Coreless Filament Winding in Academia}

\section{Completed Projects and Research}

As the benefits and limitations of CFW have been tested by the University of Stuttgart, several largescale demonstrator projects have been produced. exploring (and promoting) the potential of the technology. Key projects include:
- ICD/ITKE Research Pavilion 2012: The first pavilion produced using coreless winding, wound to create a single composite structure (Schwinn et al., 2013). - ICD/ITKE Research Pavilion 2013/2014: Created using CFW modules to allow for offsite fabrication and onsite assembly, creating a structure larger than the reach of the robotic arm (Dörstelmann et al., 2015). - Elytra Filament Pavilion: Utilising the modular approach from the 2013/2014 pavilion, this structure was the first for a prominent client in a publicly accessible location. It had to conform to local regulations and location. Ithad to contron to local regulations and typical architectural requirements of cladding foundations (Koslowski, Solly and Knippers, 2018) BUGA Fibre Pavilion 2019: Utilising a new type of modular component this recent project demonstrated modular component, his recent project demonstrated the use of fibres for creating a dome, providing a large review pro scalor structure of this size (ITKE 2019).

Supporting these larger structures, several smaller CFW prototypes and experiments have been completed, by institute researchers and as part of ITECH Master's thesis projects, such as the 2018 Bis 'Resistant Filigrees' by Jorge Christie. Beyond Stuttgart, interest in the CFW method has been demonstrated by a number of other academic institutions, highlighting a common interest in this form of material placement C-Lith (Wilcox and Trandafirescu, 2015) projects.
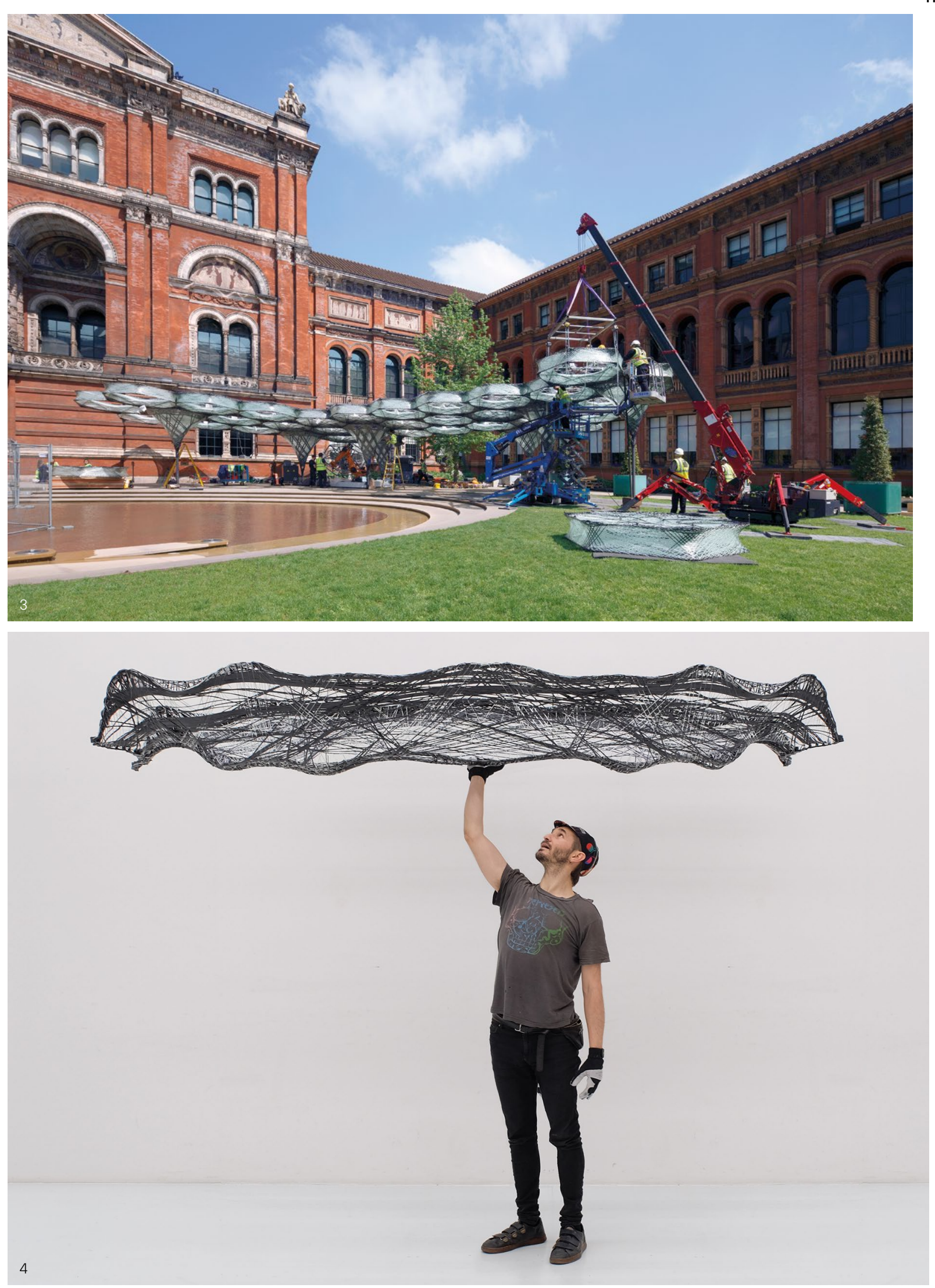


\section{Ongoing Research}

As seen in these examples, the possibility for CFW to produce novel geometries and its economical use of carefully aligned material have sparked significant interest in the academic community. While technology for the fabrication of CFW components has progressed significantly during these projects, digital tools for early-stage design and the evaluation of structura safety and integrity lag behind.

For the early design phase, key fabrication constraints must be understood to maintain the simplicity and speed of the CFW method. As geometries emerge from collisions between fibres, without an easily-usable design tool to model this behaviour, projects to date have relied on physical prototypes. The Innochain project 'Virtual Prototyping of FRP' was proposed to address this missing digital tool and the three years of work produced several productive steps plus multiple avenues for further work.

The topics of fabrication-informed design for CFW and evaluation of component structural capacity rem areas of ongoing development with several funded projects due to commence over the coming year.

\section{Academia-Industry Collaboration}

\section{The Need for a Fabricator}

As shown here, the process of CFW has been well proven for a range of projects, successfully demonstrating the benefits of these composite parts for architectural-scale installations. Given this success, it is unsurprising that private-sector firms have shown an interest and approached the university with requests for projects of their own. This leads to two scenarios in which a commercial fabricator of CFW parts starts to make sense

1. The client is seeking to implement a novel project and is happy tc in is hating research element Here, the development of the project can form part of the development the project can form part of the ongoing reseath in research, the worth of a university performing a Here, an industry fabrication partner becomes usefu.

2. The client wishes to purchase a structure that

leverages existing technology that has already been successfully trialled in a research project. Typically, this also means they would want a warrantied structure in line with any other building parts purchased in the construction industry. It is extremely complicated for a university to accep such a commission, this is th a fabrication company.
During the initial stages of the Innochain project it was clear that clients existed in both of these categories but there was no experienced fabricato in the market to perform production. While there are companies actively working on the development of CFW-type technologies (e.g. Mercedes Benz and Isotruss Technologies), they do not currently offer services allgned with building-scale production. To take advantage of this market gap, FibR GmbH was formed in 2016 by ICD Research Associate Moritz Dörstelmann and Textile Technology specialist Philipp Essers. Since its formation, FibR has worked on sever projects with the university (outlined below), while additionally performing commercial CFW design and production services for a series of other clients.

\section{First Steps in Collaboration}

Since the creation of FibR there have been two key partnerships with the University of Stuttgart that can be considered outstanding examples of academicindustry collaboration. These relationships are of benefit to both parties, as the University gains an immediate addition to the fabrication facilities available to its researchers along with specific fabrication knowledge, while the company (a startup deploying a new and evolving technology) gains access to ongoing research on the fabrication method it is seeking to commercially deploy.

\section{The Innochain Network}

In early 2017, FibR GmBH joined the Innochain Network as an Industry Partner of Early-Stage Researcher (ESR), James Solly, at the ITKE. This relationship was not

seeking to produce a specific project but was formed for mutual benefit in the ongoing investigation of early-stage design tools. Through this collaboration, early-stage design tools. Through this collaboration, Fibre Beam prototype and the Optimised Fibre Façade prototype. The Fibre Façade prototype utilised a series of tools Form-finding Form-finding studies determined, for each geometry. which ibres could acturly be placed. Op tomisation routines iteratively culled fibres from the form to ensure minimum materalusage, and winding-path algonthms

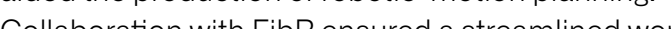
Collaboration with FibR ensured a streamlined workflow from design to engineering to fabrication, and resulted in a detailed fabrication scaffold that can be reused on future projects.

列

carbon-fibre bundles in both tension and compression, resulting in eight structural frames that weigh only $4.2 \mathrm{~kg}$ in total (the heaviest individual frame weighing just 990g).

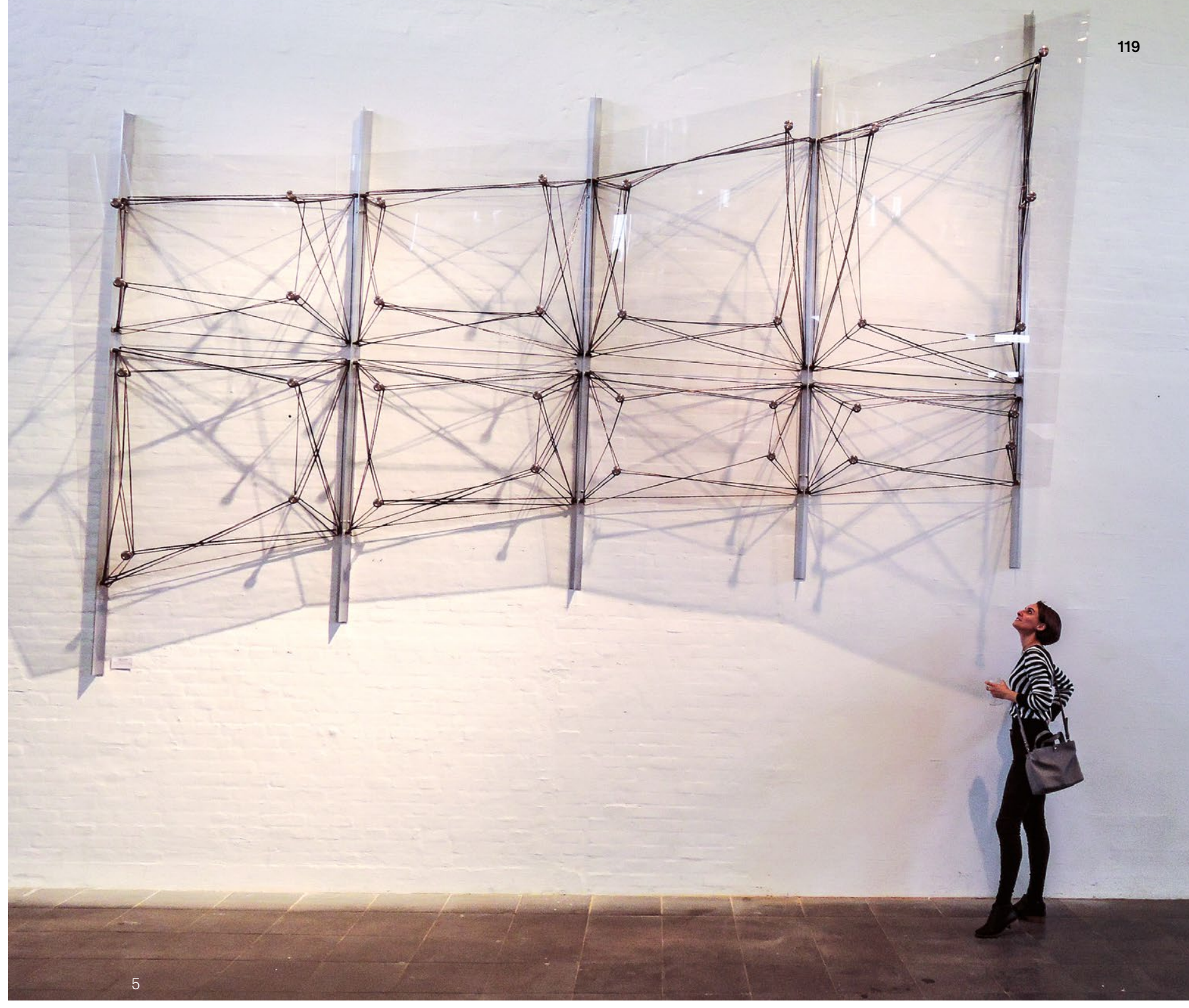



carry glass weighing twice this amount.

BUGA Filament Pavilion 2010

Between 2018 and 2019, FibR GmbH partnered with the ICD and ITKE at the University of Stuttgart on the delive of the BUGA Filament Pavilion. This is an example of a ype 1 project where the pure research elements were performed within the university with input from FibR on some aspects of fabrication. The results of this research phase were then utilised by FibR GmbH for production of the filament components.

Coreless Filament Winding in Industry

Multiple academic papers, some of which are referenced above, have outlined the proposed benefits of CFW for construction. Given these claims, the technology must now be adopted and leveraged by industry to improve the built environment if it is to be considered a success. It is therefore an exciting time for researchers involved in the development of CFW, as early projects are being produced in a commercial setting by Innochain Partner FibR. Following initial commercial production of furniture-scale items, followed by its aforementioned work on the BUGA project, FibR is currently working on a range of larger-scale commercial projects.
An interesting immediate outcome of working with ange of commercial clients has been an expansion of the material palette used for CFW. While academic research to date has selected materials based on technical performance, other clents are looking for

\section{Conclusion}

This chapter has summarised the historic work on CFW performed in academia and highlights the recentlyaddressed need for a CFW commercial fabricator. Successful academia-industry collaborations are outlined to show the benefit of having both partners working together, and some initial commercial projects are presented. Further large-scale commercial projects are known to be under active development and the first commercially-produced large building components are eagerly awaited. CFW remains a young fabrication process for the AEC industry and, it is hoped that ongoing engagement between academia and industry practice will allow it to finally demonstrate the real-world gains proposed in the research produced since 2013. Innumerable professors, researchers, students, sponsors and supporters have worked on Coreless Filament Winding and produced the information covered in this chapter. It is not possible to acknowledge every individual here and, while the bibliographical references capture some of them, all those not named are sincerely thanked for their input to this ongoing endeavour.

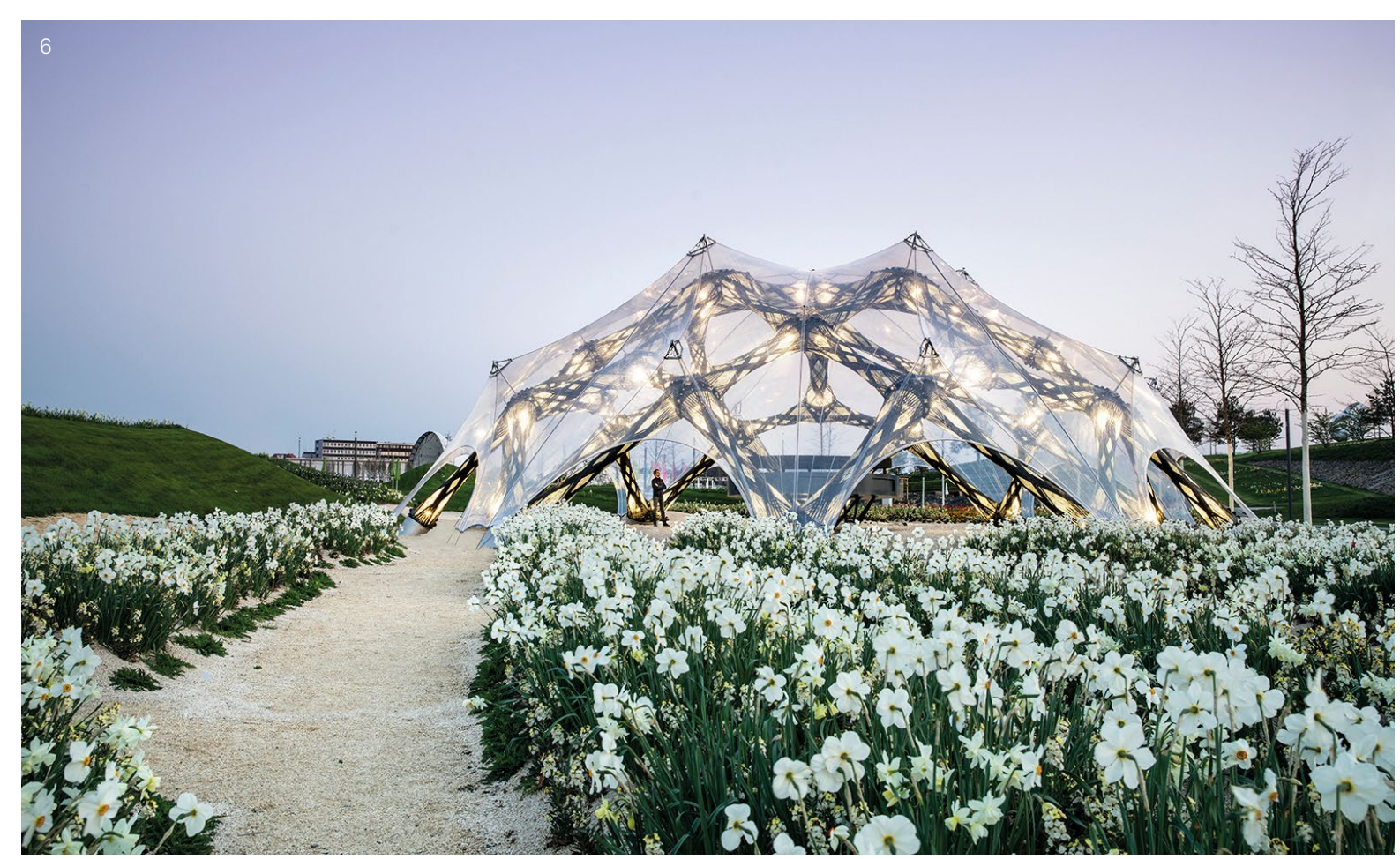

6. BUGA Fibre Pavilion 2019. ๑ ICD/ITKE, rsity of Stuttgart.

7\&8. Fibre furniture and range of winding

Bibliography Christie, ,., 2018, 'Resistant Filigrees - Shape
Optimization-to-Fabrication: Worktlow for Spanning Anplication, Stuttgart: ITECH Master's Studio.

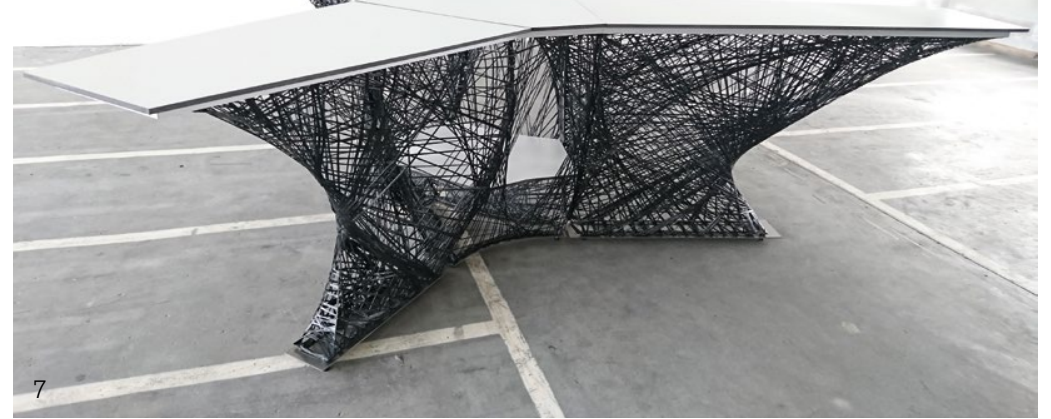

Dörstelmann, M., Knippers, J., Menges, A., "ICD/ITKE Research Pavilion 2013-14: Modular Coreless Filament Winding Based on Beetle Elytra' in Architectural Design,

ITKE (Institute of Building Structures and Structural Designn, 2019, BUGA Fibre buvilion: Www.icd.uni-stuttgart.de/projects/ 2020).

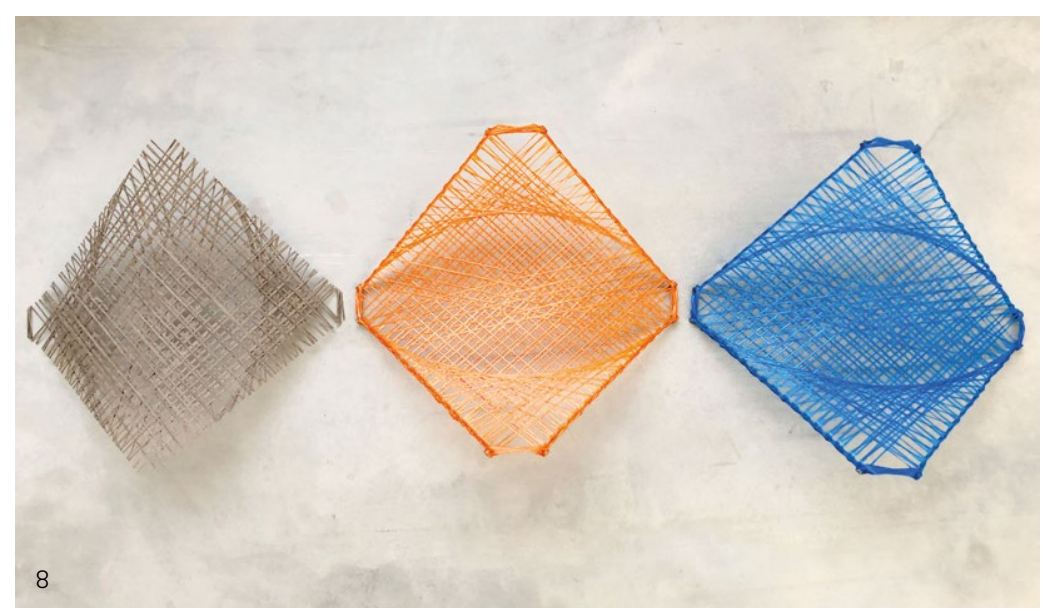

Koslowski, V., Solly, J. and Knippers, J., 2017, Based Lattice Composites for the Elytra Pavilion' in Bögle, A. and Grohmann, M. (eds.), Proceedings of the IASS Annual engineering.science, Hamburg, HafenCity University Hamburg, IASS.

Schwinn, T., La Magna, R., Reichert, S., Prototyping Biomimetic Structure for
architecture' in Stacey, M. (ed.), Prototypin Architecture: The Conference Papers,
London, Building Centre Trust, p. 224-244.

Solly, J., Früh, N., Saffarian, S., Aldinger, L., Margartit, G., Knippers, J., Structural Desig Structures, Vol, 18, April 2019, Amsterdam, Elsevier, p.1-13.

Wilcox, G. Trandafirescu A. 2015 ' $\mathrm{C}$ - Lith Carbon Fiber Architectural Units' in Computational Ecologies: Design in the Anthropocene, Proceedings of the 35th Wit, A.J., 2018, 'Cloudmagnet: A CFRP Framework for Flexible Architectures' in T. Fukuda, T., Huang, W., Janssen, P., Crolla, K.
Alhadidi, S. (eds.), Learning, Adapting and Prototyying, Proceedings of the $23 r d$
CAADRIA Onference, Beijing, p.49-58.

A




\section{All That Is Porous: \\ Practising Cross- Disciplinary Design Thinking}

Vasily Sitnikov

KTH Royal Institute of Technology, Stockholm

This chapter discusses the material systems commonly employed in the production of precast concrete elements. In particular, it presents an alternative to expanded polystyrene (EPS), a material that is often used in moulding production for complex-shaped concrete elements. Though EPS plays an important role in the production of an energy-efficient built environment, the ecological implications of its growing global use, and inevitably growing waste, raise serious environmental concerns. Putting the industrial policies of EPS use under question, the author proposes the concept of an alternative ice-based material system for concrete manufacturing. This method provides a waste-free, closed-loop recycling manufacturing waste-free, closed- the proyolion manufacturing formally rich structural formations in concrete for example mesoscale trabecular concrete structures spatial material organisations that exceed the scale spatian materialorentrotions but axe much smaller than the desirn detailing of the concrete semants. Initially intended to merely eliminate production waste, ice-based concrete manufacturing is a rion waste, ice-based concrete manufacturing is a relatively little-explored field in materlal organistion. This nove opportunity to producereduced-weight concrete elements with diferential local physical properties and expressive formal language is a welcome side-

\section{Energy Efficiency}

One of the exhibitions that took place at the 2019 Chicago Architecture Biennial was called All That is Solid. The concept was to bring approximately $28 \mathrm{~m}^{3}$ of EPS debris from a landill into the gallery space, reflecting on 'our shifting cultural and economic definitions of waste and worth, resource and refuse' $(\mathrm{L}$, 2019). EPS is one of the most ubiquitous materials, with the most mismanaged waste culture, but why would an exhibition about architecture criticise a material that is widely associated with disposable cups, tableware and product packaging? The reason is that the EPS we casually encounter in our daily lives is just the tip of the iceberg. Due to its low cost and versatility, EPS has become deeply rooted in the building industry, fulfilling many useful functions, both permanent (e.g. insulation and backfill in landscaping) and temporary (e.g. formwork and moulds for cast concrete). The waste produced in all of these applications is less obvious to the end-user as it remains obscured from public view. However, recent research suggests that construction materials contribute to the ' $99 \%$ of plastic debris by weight entering the 'o the which are 'more likely to contain chemical ocean 'which are 'more likely to contain chemical Despite the environmental hazards the pursuit of an energy-efficient built environment means EPS is wid energy eficient built umbodied energy value-ie. energy invested dung encrication and transportation. Accorling to data from the Inventory of Carbon and Embodied Energy from the In is equal to approximate is equal to approximaty the same amount of energy is needed to heat $1 \mathrm{~m}^{2}$ of residential space in Norway over a period of 25 years (Minea, 2016). Therefore, a house designed and insulated according to the sustanable concepts of 'passive or 'zero energy' (a house that uses a lot of EPS insulation) means investing a great amount of energy during the construction stage. In turn, over a period of 80 years, such a house would win merely $7 \%$ of energy savings compared to a house with outdated insulation

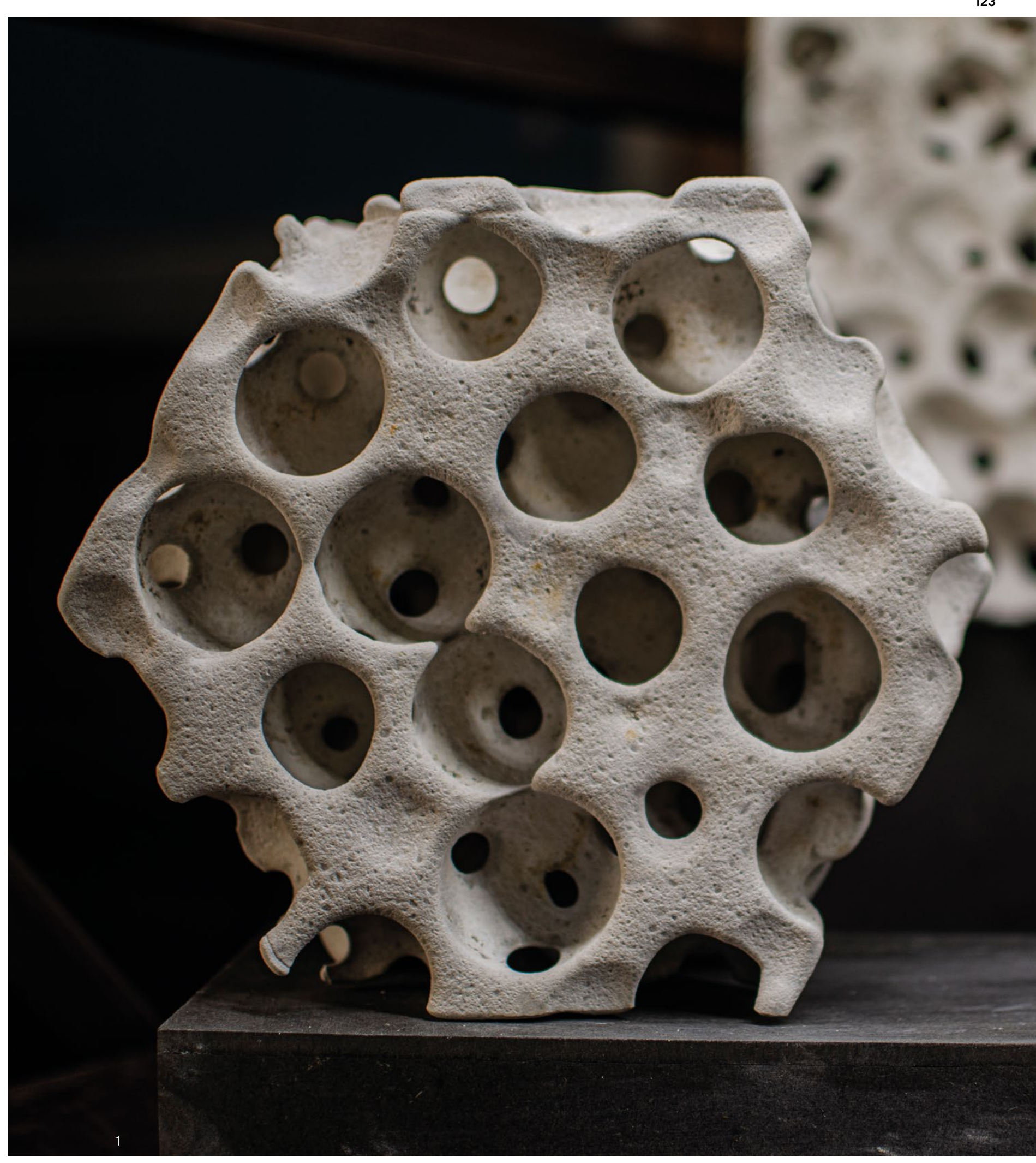

1. A hexagonal prototype of a trabecular
HPFRC structure cast using ice aggregate. 


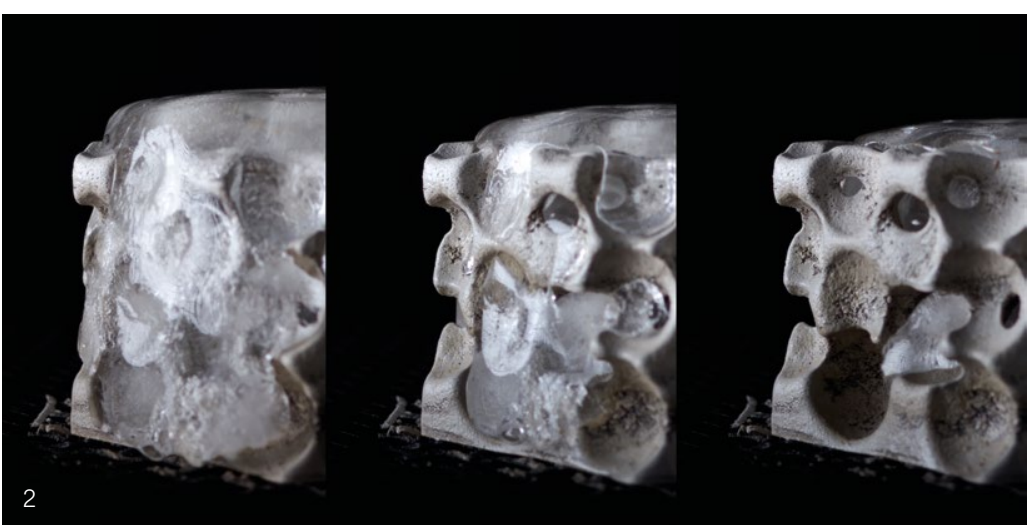

standards, as a recent lifecycle assessment analysis shows (Kovacic, 2018). The price of this modest energy saving is increased polystyrene waste, and inevitable eventual contamination of land and the oceans.

\section{Concrete Redefined}

When examining concrete's environmental performance. one has to keep in mind that its chemistry, or more precisely chemistry of its constituent, cement, does not significantly differ from that of natural limestone. This means that its exposure to the natural environment is safe, and cannot result in an unforeseen effects, such as long-term chemical contamination. In fact, exposed hydrated cement in concrete constructions has a capacity to capture carbon dioxide from the atmosphere. This means that concrete carbonation, can be utilised to reduce the carbon footprint of concrete's manufacturing and transportation. Just as timber constructions are and transportation. redefined according to its capacity to be a carbonneutral building material.

Furthermore, the embodied energy of concrete is extraordinary low, approximately $0.75 \mathrm{MJ} / \mathrm{kg}$. Compared to the previously mentioned $88 \mathrm{MJ} / \mathrm{kg}$ of EPS, $9-29 \mathrm{MJ} / \mathrm{kg}$ of steel, or $15 \mathrm{MJ} / \mathrm{kg}$ of plywood, concrete is a strong of steel, or $15 \mathrm{MJ} / \mathrm{kg}$ of plywood, concrete is a strong
choice when it comes to laying the ground for low-energy choicewhen it comes to layng the ground for low-ener construction priples. Nevertheless, this has not been the priority for the industr, as the low price of concre means the need to optisise th use is not pressing. Instead, the industry continues to build excessively thick concrete constructions, since the man function given to concrete is to protect steel rentorcement fom corrosion. How wer his material con iguration is farless sustainable then was originally expected. The long-term exploitation of exposed concrete constructions has shown that diffusion of the atmospheric carbon dioxide in concrete rapidly propagates through its porous microstructures, changing the alkaline level and triggering steel oxidation. To counteract this phenomenon, the thickness of concrete used has gradually increased, resulting in excessively heavy concrete constructions. Concrete is held back from becoming a 'green' building material for reasons rooted in the conservative building codes inherited from preceding epochs. If ferrous reinforcement itself had been replaced with recently developed glass fibre, carbon fibre or other non-ferrous reinforcement concrete constructions would have lost half of their weight, and the process of carbonation would already have been considered a positive side-effect. Meanwhile, steel reinforcement is not the only aspect that constrains concrete development. It is commonly known that concrete formwork is one of the most expensive aspects of concrete manufacturing. Usually, the material used in producing the mould is steel, wood, or EPS foam. The more complicated the geometry of concrete element is, the more difficult it is to reuse the formwork, therefore more formwork material is wasted in the production process. In recent decades it has become common practice to use epoxy-coated EPS formwork for one-off concrete products. As discussed earlier in this piece, EPS waste generated by the construction industry is itself a big issue, but it is not the only issue that this material practice presents. When using steel formwork for manufacturing 1000 concrete en formwork's embodied energy is added to each cast form con concrete elements using wood based formwork, 0.1 of the wood's mbodicdenergy is add to to concrete, which is already a signilicant increase. However, in case embolied enegy of the used polystyene, which dives it from $0.7 \mathrm{Mu} / \mathrm{kg}$ up to $20 \mathrm{Mu} / \mathrm{kg}$ (although, which drives thom $0.7 \mathrm{M} / \mathrm{kg}$ up to $20 \mathrm{M} / \mathrm{kg}$ (alhough, depending on the design of the formwork, his value can vary). The main danger of this energy waste is that it is not accounted for by the actors of this production, just as still too few account, for example, hat every round trip ticket on flights from New York to London costs the Arctic three more square metres of ice 'Wallace-Wells, 2019). Ice itself presents an alternative to this material

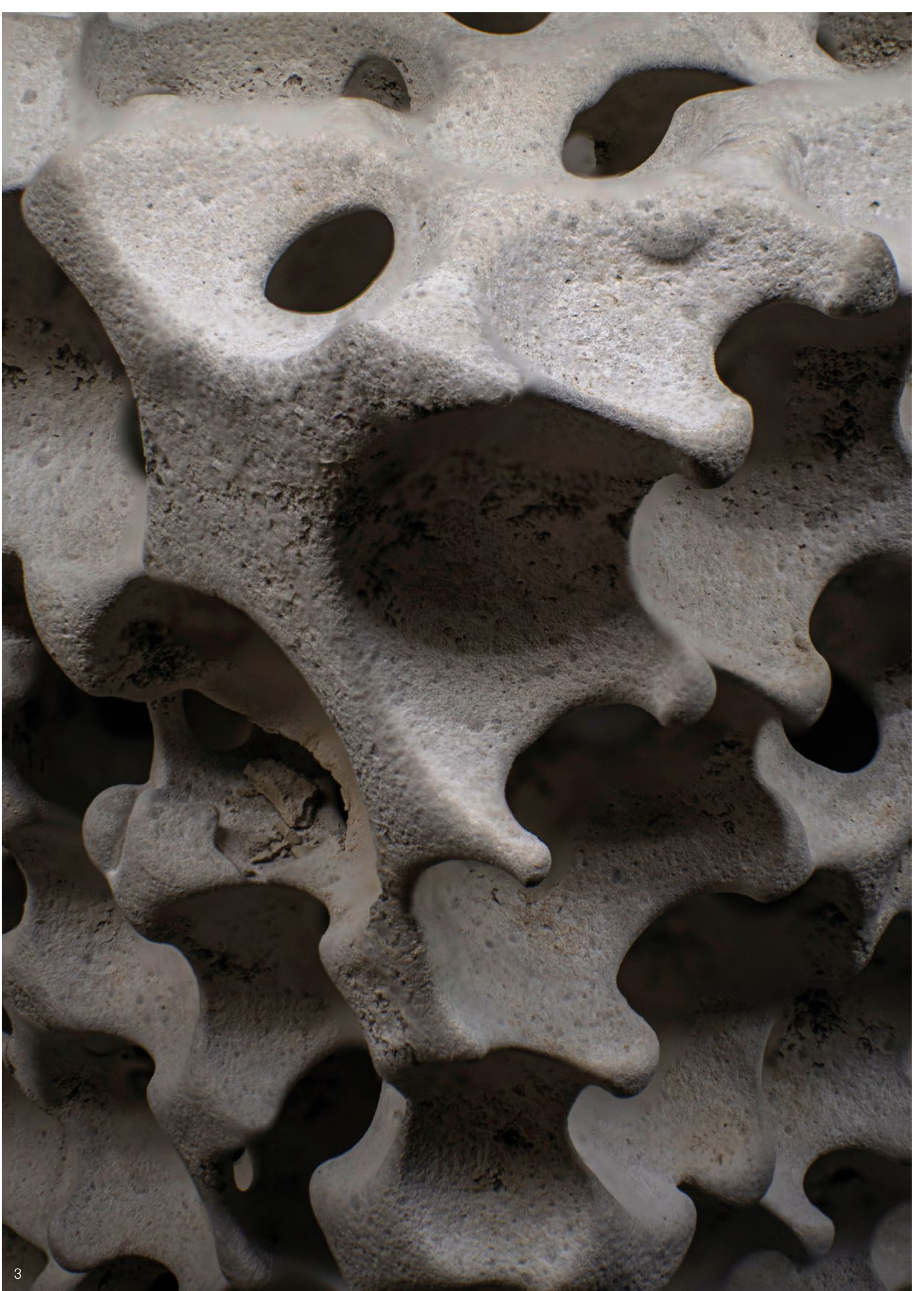

125 practice that has been developed in the author's 


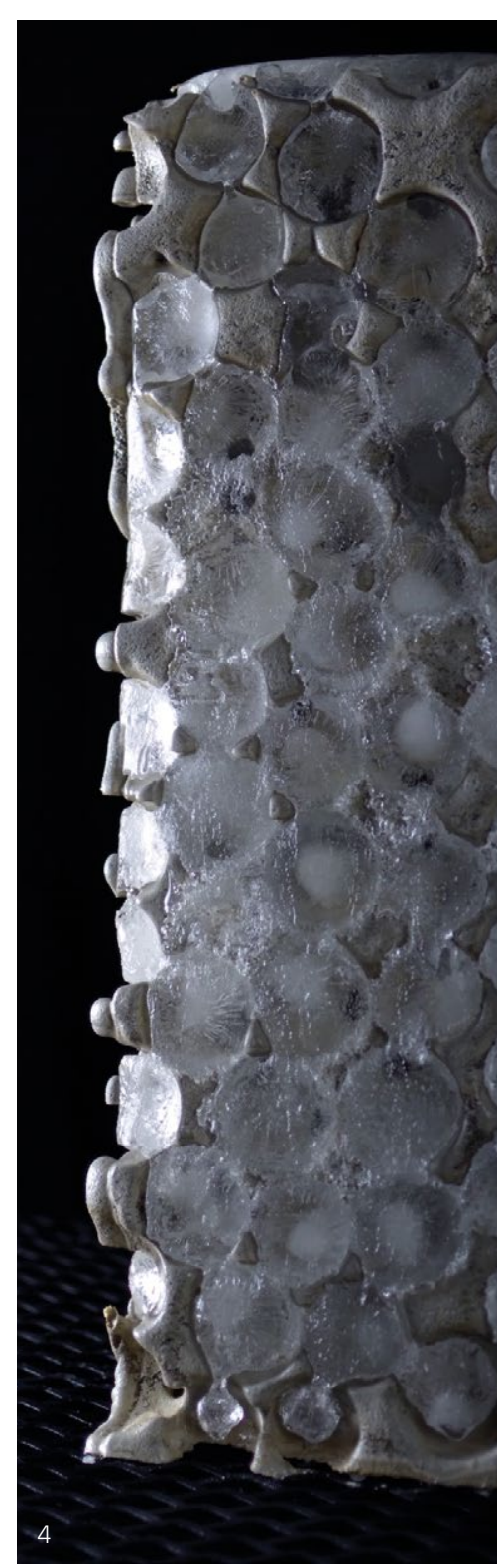

research into methods of using ice as the formwork material for concrete manufacturing (Sitnikov 2019, Sitnikov et al. 2019). Hsingle-use EPS formwork is substituted with ice formwork, the production of the same concrete element would result in drastically different embodied energy values. Instead of $20 \mathrm{MJ} / \mathrm{kg}$ of concrete made with EPS, the ice formwork process would add only $10 \mathrm{MJ} / \mathrm{kg}$ of concrete if, for example, it used an artificial refrigeration system during summer. At the same time, it will eliminate all solid waste and automate the process, since the ice formwork is self-demoulding and water used in the production of ice can be indefinitely reused.

\section{Mesoscale Trabecular} Structured Concrete

The prototypes featured in this chapter showcase spatial patterns that can be imposed on concrete, abandoning any use of petrochemicals in the fabrication process. Breaking away from the 'solid' image of conventional concrete, the technique of using ice as the formwork material enables the production of mesoscale spatial structures in concrete which would be impossible to manufacture with existing formwork materials. While opening doors for new design explorations, such a spatial structure of concrete is, in principle, a new. composite material. The concrete used in this cast belongs to the class of high-performance concrete, with compressive strength over $100 \mathrm{MPa}$ and density of $2400 \mathrm{~kg} / \mathrm{m}^{3}$. However, when formed into such a structure, its bulk volumetric density drops to $820 \mathrm{~kg} / \mathrm{m}^{3}$, maintaining its surface density at very high levels. The variety of programmable functions for ice formwork is vast across environmental design, programmable lighting conditions, acoustics, ventilation, insulation and structural-design weightsaving applications. An existing precedent of simila material organisation in architectural design can be found in one of OMA's projects. In 2002 the office was commissioned to design a new Prada teal stre in Beverly Hills, LA. The design team crossed disciplinary boundaries and ventured into material programming and physical prototyping, developing an industrial fabrication

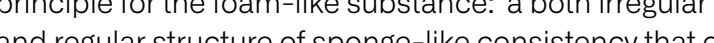
and regular structure of sponge-like consistency that can be cast in stages from hard to soft and from transparent to opaque' (OMA, 2002). Chemically, the sponge was made of polyurethane cast in silcone moulds to form a random yet carefully-controlled volumetric pattern. The material itself is relatively expensive, so the foam did not enter mass-use. In fact the OMA FOAM walls of the Prada store are enclosed in glass cases, just like exhibits in a museum, highlighting that this is essentially a
full-scale prototype of a building material.

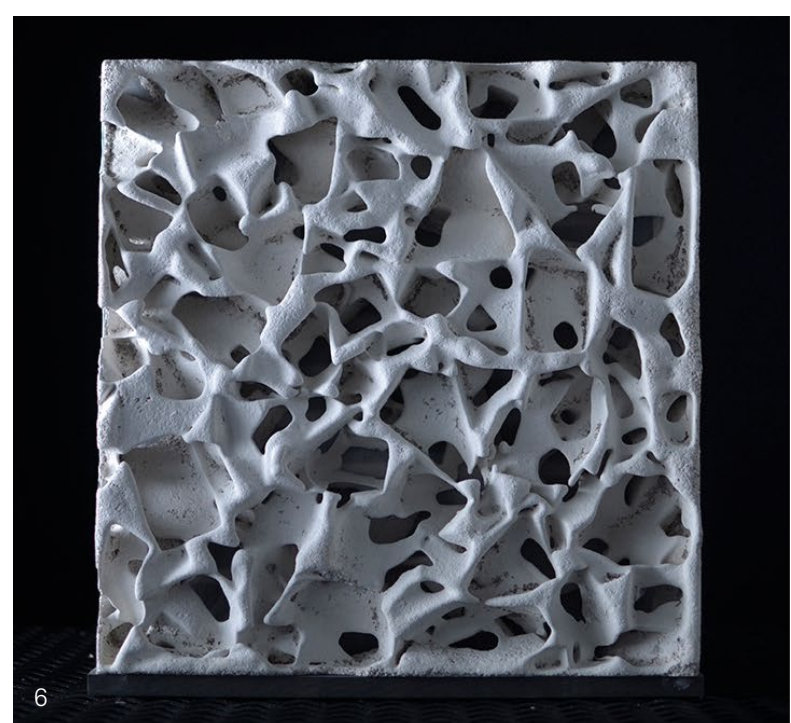

Yet the material configuration of ice-based concrete casting produces a similar effect at much lower costs. Moreover, borrowing a concept from Sylvia Lavin that refers to a geometrical language translated from one material into another, that is 'wood and wood recollected' (Lavin, 1992), the trabecular concrete structure can be seen as a recollection of OMA-FOAM vision. That is, the phenomenon of OMA-FOAM, once an artificiallysynthesised visionary object, today finds a natural way of materialising through a sustainable configuration of concrete and ice. In fact, the method of recollecting synthetic materials in a natural medium could serve as a reliable vehicle on the way toward sustainable living. By taking a step away from the dogmas inherited from an earlier social and economic epochs, and questioning the foundations of material processes, this research investigation has led to potentially rewarding design investigation has led to potentially rewarding design like this should be made a least in architecture, steps like this shold be made aw environmentally and socially fair technologies.

4. Demoulding/defrosting of a HPFRC pillar prototype.

5. A prototype of FOAM by OMA at the
demoulding stage. Courtesy of OMA photo: Phil Meech.

6. Light permeability of trabecular concrete structure (irregular crushed ice aggregate

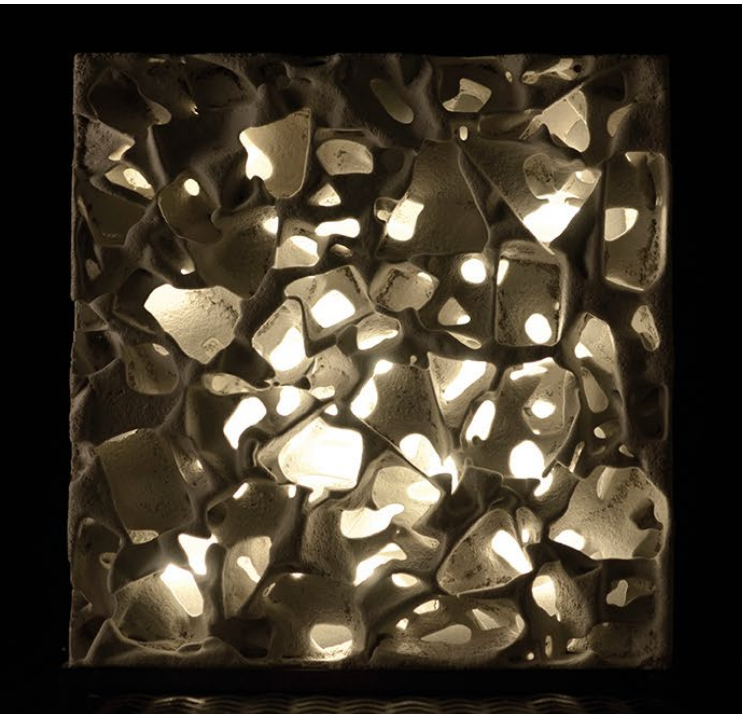

Bibliography

De Frond, H.L., van Sebille, E., Parnis, J.M. Diamond, M.L., Mallos, N., Kingsbury, T. and Rochman, C.M., 2018, 'Estimating the Mass
of Chemicals Associated with Ocean Plastic Pollution to Inform Mitigation Efforts' in Integrated Environmental Assessment
$\&$ Management, Vol. 15, No. 4, p.596-606

Hammond, P.G. and Jones, C., 2011, And Energy (ICE)': The Inventory of Carbon Engineering, University of Bath \& BSRIA http:// whw.circularecology.com/embodied-energyXhy-ay2cbzll (accessed 13 January 2020).

Kovacic, I., Reisinger, J. and Honic, M., 2018 Operational Energy for a Passive Housing Block in Austria' in Renewable \& Sustainable
Energy Reviews, Vol. 82, Part 2, p.1774-1786. Lavin, S., 1992, Quatremère de Quincy
and the Invention of a Modern Language
of Architecture, Cambridge (MA), MIT Press, p. 111 .

Li, A., 2019, 'All That Is Solid' (exhibition annotation).https://space-p11.com/ all-that-is-solid (accessed 4 October 2019). 'Canadian Low-Energy Housing. National' Energy Context, and a Case Study of a Demonstration House with Focus on its Technology for the Bult Environment No. 4, p.651-668.

OMA, Prada Sponge (project). https:///oma.ew projects/pr.
2019).

Wallace-Wells, D., 2019, The Uninhabitable Bandom House / Tim Duggan Books. 
Research Summary

Bend\&Block: A Passive

Form-Giving Strategy

Efilena Baseta

Industrial partners: Blumer Lehmann AG, Foster + Partners

Academic Institution: Institute of Architecture, University of Applied Arts Vienna
Adaptable architecture has become a trend in the digital era as a sustainable response to the rapidly changing environment and fluctuating user preferences. Shapeadaptation, in particular, constitutes a key characteristic in 'smart' shading systems, multipurpose spaces and the like. The majority of such systems entail kinetic facades, which change their permeability, or photovoltaic panels, are, however, limited in scale, despite the sustainable solutions they offer, as they require a lot of energy to perform and are structurally challenging.

This research seeks to identify innovative solutions to develop large-scale, shape-adaptable structures.

The research methodology is based on empirical The research metho evidence and know colge, via direct and indirect hypothesis, verified by existing laws and tested by physical and disital experiments. In this context physical and digltal experiments. In this context, scales led to the invention of a passive, scalable, scales led to the bending active system of controlled deformations. that was chevelo is the key characteristic of the syste tested through physical experiments with various protolypes. Digtal fabrication techniquesweremacro-scale, while these physical models wereused as an analytical tool to venify the functionality or the system. The experimental data that was collected was analysed and compared with the data from digttal and physical simulations. The digital models served as an exploratory tool that not only explained the structural performance of the physical models but also generated new ideas which adapt their orientation. Shape-adaptive systems The study of transformable mechanisms of various that employed to createprotypes ona micro-, meso-and for further exploration. Finally, conclusions were drawn regarding the performance of the system, by combining the findings from both the digital and the physical experiments.

Given that this work sought to develop a novel construction system, collaboration with leading-edge of a transformable roof structure were carried out, together with Blumer Lehmann AG, using a realistic framework based on academic research. Fabrication was conducted with a Hundegger K3 - an industrial milling machine optimised for the rapid machining of long, straight timber beams. The industrial fabrication of the system indicates its potential to be massproduced and to find applications in macro-scale $(>10 \mathrm{~m})$ structures. A further collaboration, with Foster

The active-bending system of controlled deformations explored in this research is known as Bend\&Block. The structural behaviour of this system relies on the The structural behaviour of this system relies on the joinery detalls between consecutive layers of multilayered structural parts. In contrast with traditional, mechanically laminated beams, small transversal gaps the role of a gometrical swich between two states flat and the and gradually close during bending. In the Hat state, the element is flexible and susceptible to deformations. Once the gaps are closed, the stiffness of the system. increases instantaneously and blocks at a specific form. The two-stage stifness, embedded in the construction details of the system, enables controllable deflections when loads are applied.

To conclude, Bend\&Block is a passively-activated form-giving process, with applications in transformable industrial partners was crucial. The design and fabrication + Partners, enabled 3D-printed transformable plates micro-scale $(<1 \mathrm{~m})$ to be exp are inserted betwe the lays. These gaps perform
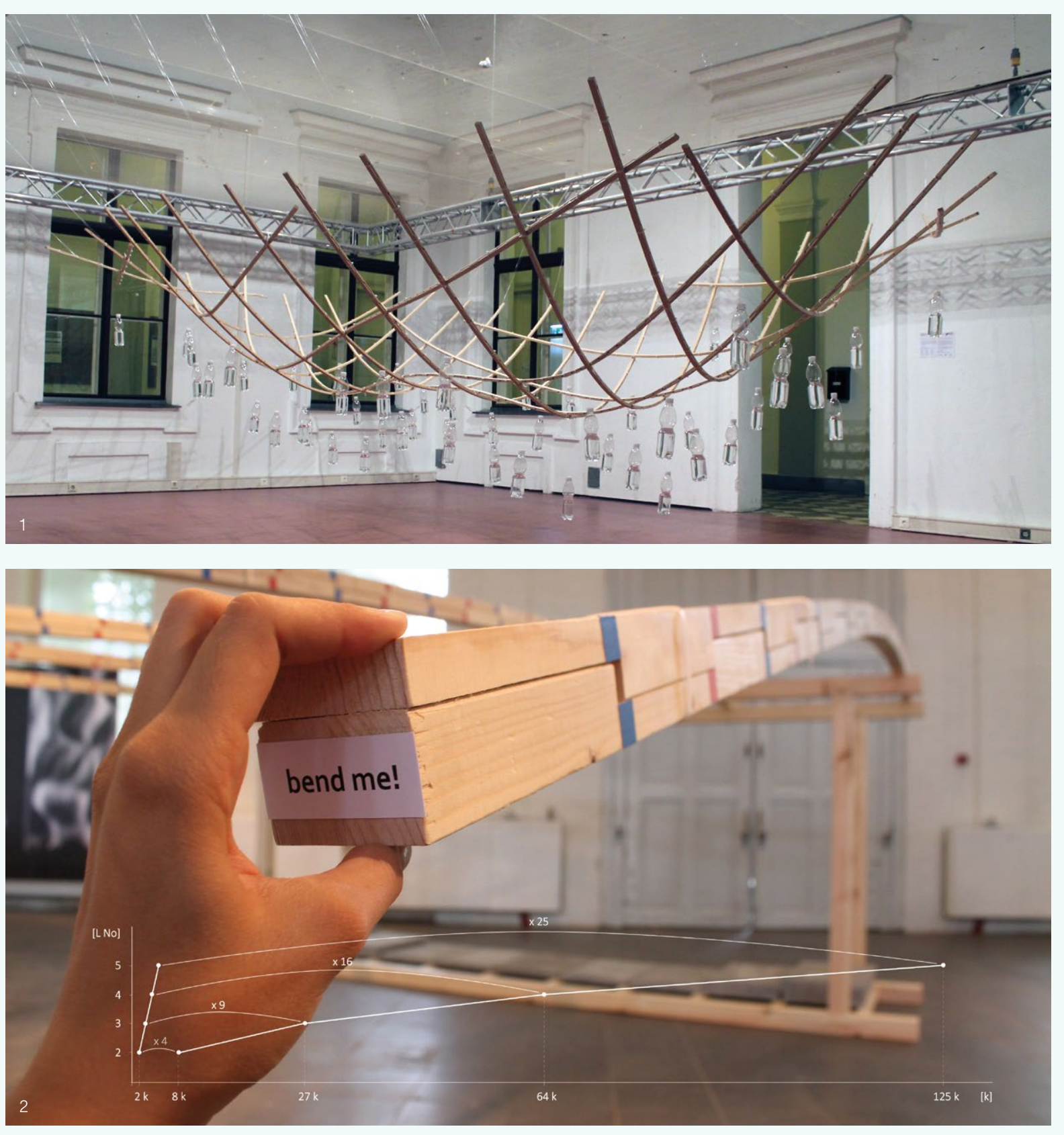

active-bending gridshells and plates, such as

Acknowledgements

shape-adaptable roofs, bridges and façade elements. The analysis and evaluation of the structural behaviour of various prototypes and demonstrators, at different scales, have proven the functionality of the system. Supplementary physical and digital experiments, however, need to be conducted to scale-up and industrialise the system discussed here.

The digital fabrication of the mesoscalar timber prototypes would not have been possible without the support of Angewandte Robotic University of Applied And Vienna, within the framy Laboratory of the Design and Full-Scale Fabrication seminars 2017 and 2018, led by Andrei Gheorghe.

1. Double-curved gridshell structure which consists of flat fabricated
double-layered laths with embedded shear blocks This system can ind application in transformable roofs activated by water/snow

bads. Photo: Efilena Baseta

2. A double-layered beam of the Bend\&Block system. The graph illustrates the stiffness jump from the flat state to the bend state in 
130

\section{Research Summary \\ Adaptive Robotic Carving}

Giulio Brugnaro

Industrial Partners: Bjarke Ingels Group, Rippmann Oesterle Knauss GmbH

Academic Institution: The Bartlett School of Architecture, UCL

In recent decades, digital fabrication technologies have become increasingly available, yet manufacturin knowledge is rarely integrated within the established workflows of design practices. Materialisation processes are regarded as the last stage of design-to-manufacturin workflows, where materials are considered passive receivers of a previously-generated ideal form, stored in a digital model. Such linear progression from the design intention to its materialisation necessarily limits the feedback between different stages of the process, forcing designers to engage with only a limited range of standard manufacturing methods and materials. which often leads to wasteful and inefficient solutions. The complex interaction of non-standard tools, such as chisels and carving gouges, with the heterogeneous properties of timber, cannot be ignored within robotic manufacturing as it substantially affects the resulting carved geometry. The central challenge addressed in

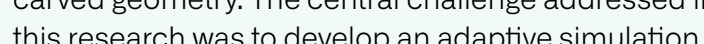
model that could bridge the diritally-prescibed design intention and the outcome of the carving proced desig The research proutcome of the carving process. The research mpoposed a series of mehods to capture, transfer, augment and integrate manufacturing knowledge at an ealy stage of the design process through the collection of real-world fabrication data, using different sensor devices and machine leaning models to achieve an accurate prediction of carving geometries, informed by materia behaviours. The acquisition of fabrication to was structured by a series of recording sessions to store, in a library of datasets, the combination of fabrication parameters and respective operation outcomes generated by different material properties (e.g. grain structure, density, direction), wood species and carving tools. The collected datasets can be used whose main learning objective is to predict the carved ometry generated by a user-defined robotic toolpath and a series of fabrication parameters.

Collaborations with two industry partners - ROK Architects, Zurich, and BIG, Copenhagen - provided the opportunity to apply the methods we had devised into established workflows and develop a catalogue of design explorations for a wide range of applications, from furniture to building components of larger assemblies. The curation of the training process, by a team of designers, represents the keystone of the design workflow, as the selection of relevant materia affordances and fabrication parameters directly determines the range of solutions later available in the digital design exploration. In this way, the trained system represents a package of knowledge that can be integrated within an interface to digitally evaluate multiple, otherwise unavailable, design solutions informed by tools and material properties, before moving to the
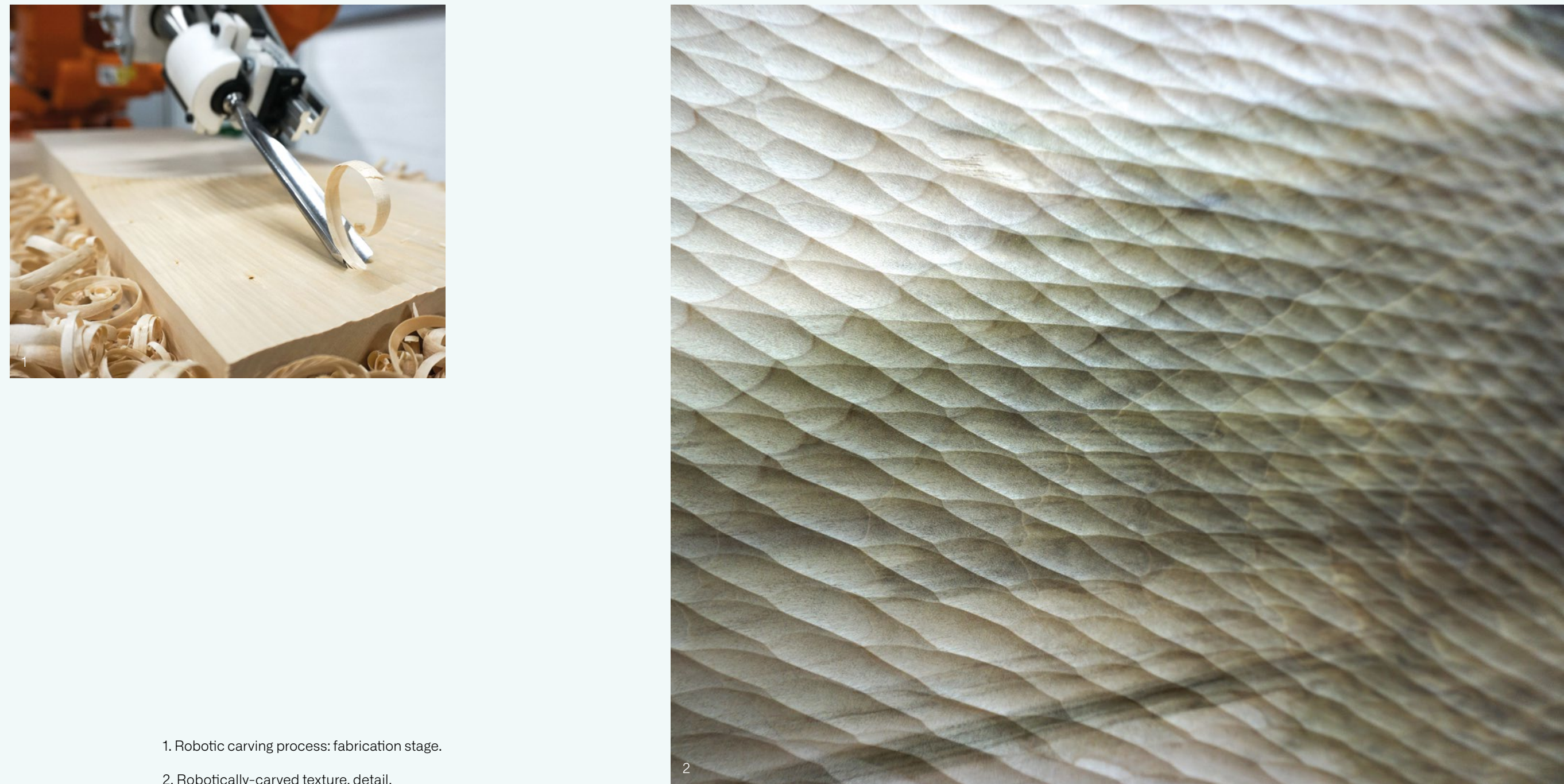

production stage. Once robotically fabricated, a deviation analysis with the respective simulations, to assess the predictive abilities of the system. The results successfully demonstrate the ability of the trained networks to accurately model the outcome environment by a series of fabrication parameters. The impact of the research lies in devising a series of robotic training methods that allow for flexible extensio of the range of subtractive manufacturing processes available to designers. Novel design opportunities are explored that support decision-making procedures, based on an accurate simulation of non-standard operations on timber. The integration of manufacturing knowledge at the early design stage allows for a custom design-to-manufacture workflow to be established, informed by continual feedback, that encourages a fruitful dialogue between designers and manufacturers. the selected carved geometries are compared in of carving operations, defined in the digital design 


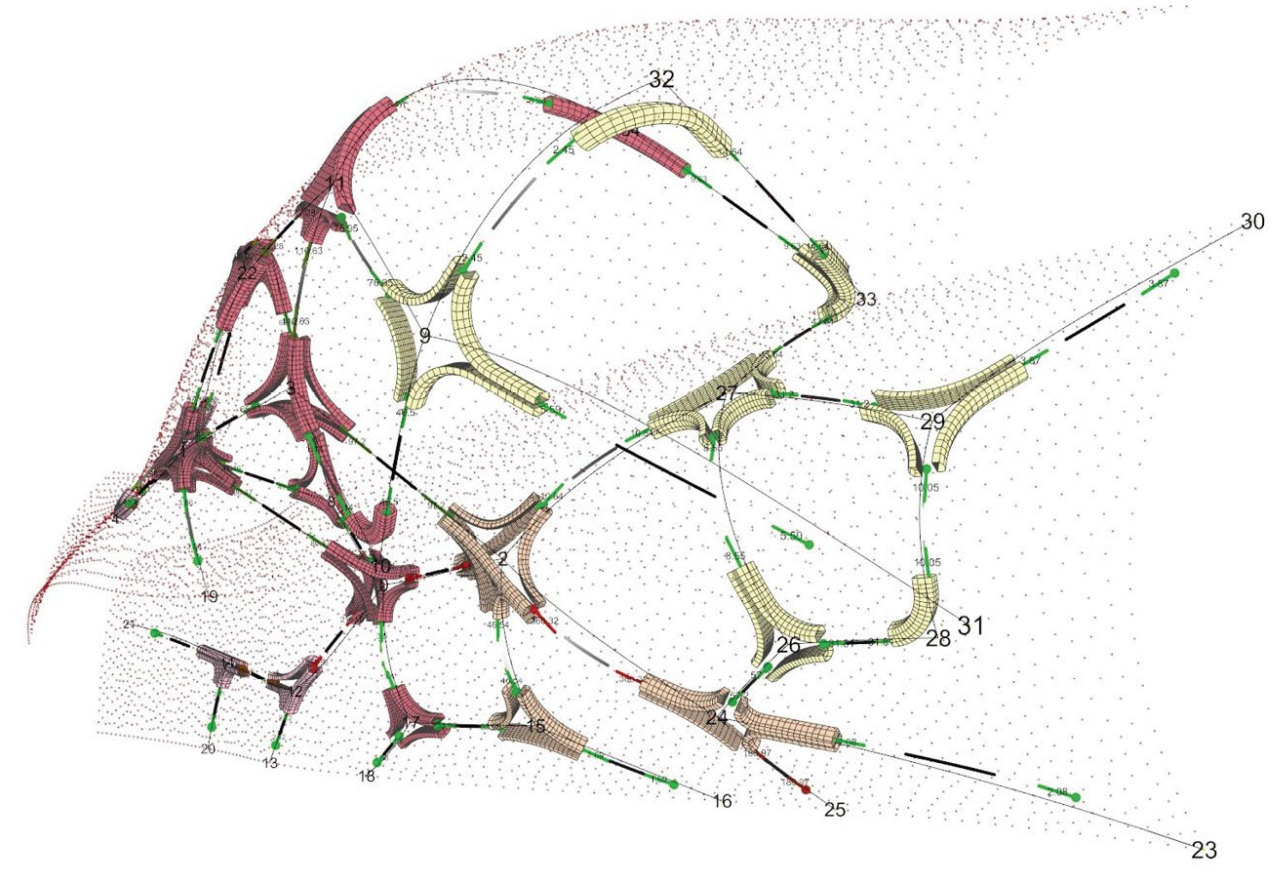

successful design process. In some cases, the chosen tools, although readily deployed, reinforce bad design script-creation, or do not support graduated transition from simple computational concepts through to the more advanced solutions in a code environment. It is also difficult to reconcile differences within teams, between VP environments and a more formalised and generalised code environment, and successfully enable advanced computer programmers or software engineers to collaborate meaningfully with less experienced practitioners, in a standard VP environment.

Therefore, to address these challenges, a transparent, extensible and modular design and collaboration system was developed, on both the conceptual and the technica level. The first stage of this project presented the management of non-linear disital workflows the intricate datasets at late stages in the design process of complex architectural projects. The second stase proposed alternative concepts, tools and methodo to clarify and improve the existing proceses used in the to CCC industry. The e e illustrated in the third stage by AEC industry. These are llustrated in the third stage by means of different empirical experiments grounded in the existing everyday practices of BuroHappold partners of the Innochain research network and supervisors of the author.

The experine author. The experiments make use of a diverse range of interdisciplinary concepts borrowed from computer science and data visualisation: schema-based wrich

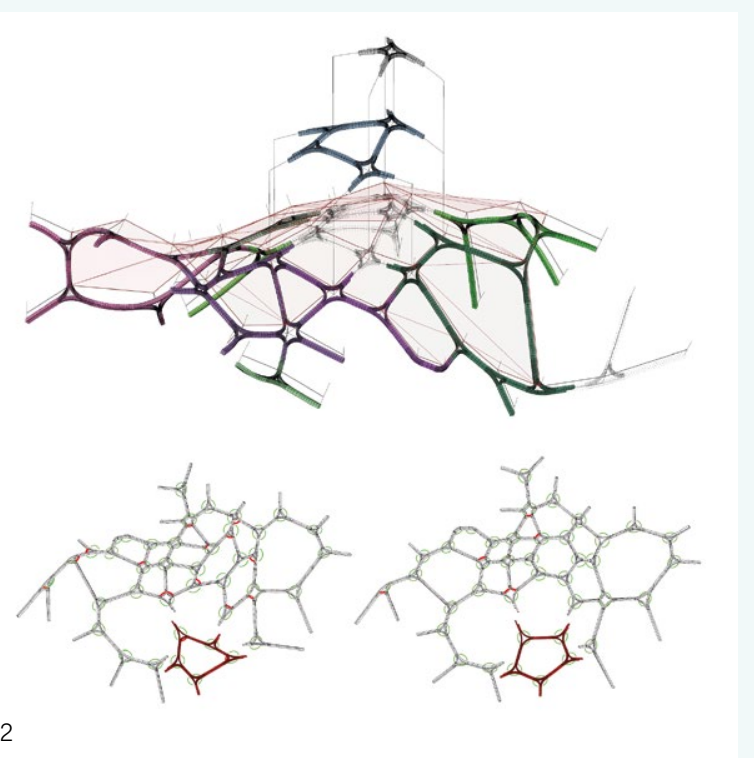

abject Model (BHoM) - a computational design, industry - and Speckle - an extensible design and AEC data-communication protocol and platform, initiated by Dimitrie Stefanescu within the Innochain research network (see also p.62-67 and p.80-81 of this volume). The research concludes by reflecting on the experiments developed so far, extrapolating their potentials and veculating on a broader theoretical framemork the enables co-creation and mass participation.

\section{Bibliography}

Scheurer, F., 2012, 'From Thinking to Modeling to Building' in Marble, s. (ed.), Digitial Workfflows in Architecture: Design-Assembly-

Speckle: Open Digital Infrastructure for Designing, Making and

The Buildings and Habitats Object Model: Sustainable Code
at Scale, 2018. https://bhom.xyz (accessed 7 February 2020)

van der Heijden, R., Levelle E. and Riese, M., 2015, 'Parametric Building Information Generation for Design and Construction in Computational Ecologies: Design in the Anthropocene:
Proceedings of the 35 th Annual Conference of the Association for
Computer Aided Design in Architecture (ACADIA), Cincinnati, Ohio http://papers.cumincad.org/data/works/att/acadia15_417.pd
Deutsch, R., 2017, Convergence: The Redesign of Design Derating the Built Environm
Topological mapping: spatial branching.

2. Topological mapping: projection-based modelling search interfaces used to ease and enable the assemb

$\begin{array}{ll}\text { particularly the widely adopted VP environments, do not } & \text { visualisation and querying of complex datasets at late } \\ \text { natively or explicitly encourage collaborative design } & \text { stages in the design process. These experiments rely on }\end{array}$ two in the design process. These experiments rely on behaviours, suchy encourage collaborative design 


\section{Research Summary}

Ice Formwork: Challenging

the Sustainable Production of Non-Regular Geometry in Concrete

Vasily Sitnikov

Industrial Partners: Buro Happold

Academic Institution: Royal Institute of Technology (KTH), Stockholm

The goal of the research is to provide a vision of innovative, environmental and efficient developments for both the design and manufacturing processes of precast cement-based components. A survey of conventional construction technologies, industrial standards, and latest developments in digital fabrication and the material science of concrete provided a list of initial criteria for innovative concrete design.

First, the survey showed that precast concrete production is preferable in comparison to in-situ-cast concrete production. The combination of controlled and equipped environment, skilled labour and the stable logistics associated with precast concrete all contribute to a better use of time material and energy. Second the survey identified the potential of recently-developed high-performance concrete (HPC) in reducing the weight of constructions, while providing the same leve of structural capacity Lighter constructions deliver savings on transportation and raw materials (Sitnikov, 2017). Third, the use of non-ferrous fibre reinforceme presented significant benefits over traditional steet presented significant benefits over traditional steel reb corrosion and requires less manual labour.

Design Features

In collaboration with engineers from BuroHappold Engineering in London, the internal structural principle of a fibre-reinforced precast HPC element was researched. If applied as a rain screen on a façade, such an element should span at least $3 m$ to be viable for uses spanning Hoor-to-floor heights. While aiming at a significantly reduced mass, the target thickness was identified as
$3-4 \mathrm{~cm}$. Considering the wind load and possible impact, and the absence of rebar reinforcement, the element would require an integrated ribbed grid at the back to maintain its structural continuty. Due to its slender form, such a design would have a very narrow dimensional tolerance and, if cast, would require a two-componen formwork: a mould and a counter-mould.

Materials and Process

To maximise the formal potential of this design, the production method needed to be able to cope with a maximum amount of geometric variability and element-uniqueness. One conventional fabrication method, suitable for production of such a design, uses CNC-milled moulds of a solid and low-density material, such as Expanded Polystyrene (EPS). The efficiency and versatility of this approash have been proven in practice, including Neuer Zollhoff by Frank Gehry (Kolarevic, 2003) and Fjordenhus by Studio Other Spaces (Sendergard and Feringa, 2017). Despite its merits, howard and Feringa, results in large volumes of non-recyclable waste and therefore, requires improvement. With the and, therefore, requires inprovement. With the aim of achieving a more efficient fabrication process

In the first phase of development, a frost-resistant design of high-performance concrete was created. In collaboration with two research institutes, a concrete cond coration with two resch designed (Sitnikov and Sitnikn, 2018) In the was designed (Sitnikov and Sitnikov, 2018). In the second phase, numerous experments were performed to test the CNC-machining of ice, the rheological properties of HPC, autonomous demoulding through natural ice-thawing and production of low-defect ice blocks (see Bibliography for video of the process).

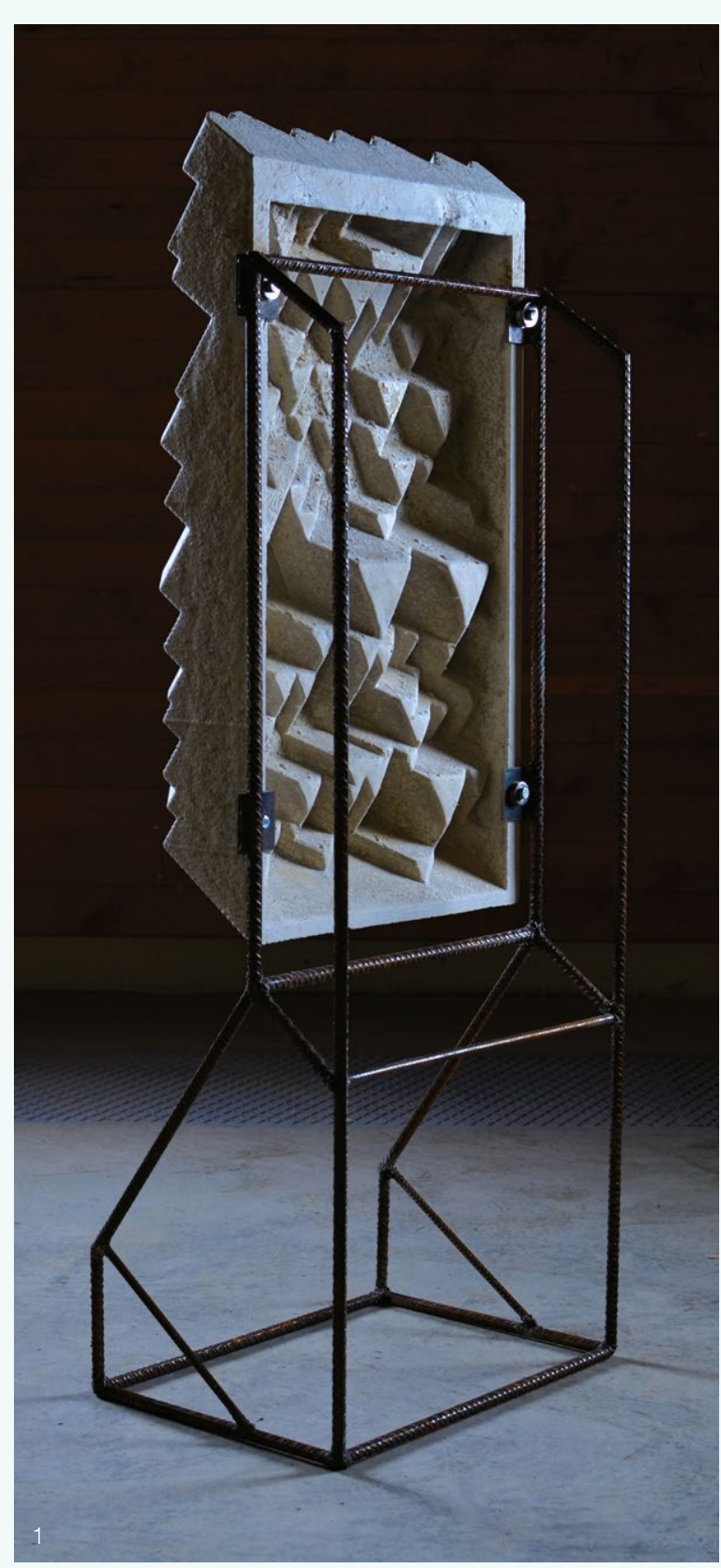

The prototypes illustrate the quality of the concrete casts and lend themselves to complex geometry independent from manual labour. Since water can be thawed and frozen an infinite number of times, this production method can function in the form of a closed loop, relying only on electricity supply. Moreover, preliminary assessments have shown that the energy consumption of ice production is outweighed by the overall energy savings (Sitnikov, 2019).

Bibliography

Kolarevic B., 2003, Architecture in the Digital Age: Design and Manufacturing, Hoboken, NJ, Taylor \& Francis Limited.

KTH School of Architecture, 2018, "Ice Formwork: An Ice-Based CAM Concept for Precast Cor

Sitnikov, V., 2017, Ilce Formwork for Ultra-High Performance Concrete: Simulation of Ice Melting Deformations' in de Rycke, K., Gengnagel, C., Baverel, 0., Burry, J., Mueller, C... Nguyen, M.M., Rahm, Modelling Symposium, Paris Singapore, Springer, p.395-406.

Sitnikov, V. and Sitnikov, I., 2018, 'Kinetics of UHPC Strength Gain at Subfreezing Temperatures: SP-326 Durability and Sustainability of

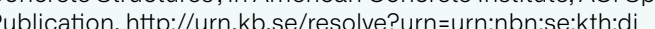
Va-247458 (accessed 8 February 2020).

Sondergaard, A. and Feringa, J., 2017, 'Scaling Architectural

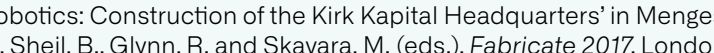
UCL Press, p.264-27.

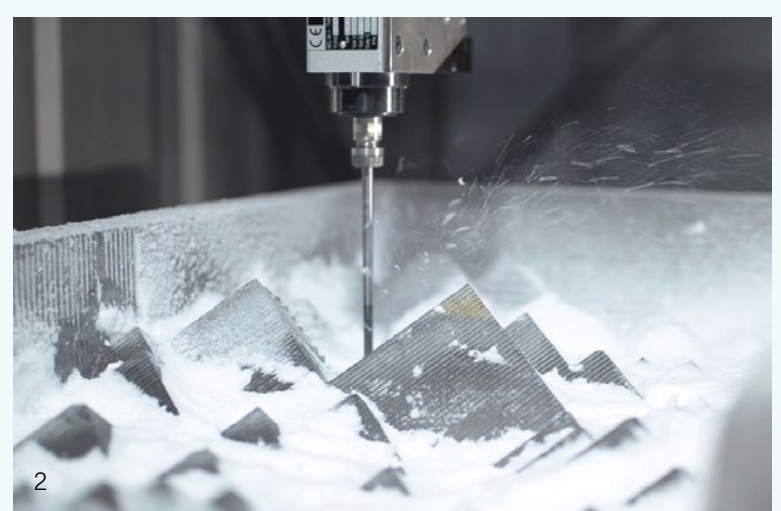
lightweight High-Performance Fibreacade, formed with an ice c) rain-screen 2. CNC milling the ice mould using a $6 \mathrm{~mm}$ 
The development of the digital tools and workflows or CFW virtual prototyping was performed with an experiment-based methodology, where promising ideas that emerged from the initial study phase were tested - first digitally, under simplified conditions, and then physically, during collaborative large-scale projects and research-specific smaller prototypes. This work stage initially focused on using pre-existing, open-access to and libraries, selecting those with potental selevance the winding method, then testing them as described. Early on in the work, it became clear that some custom tools would be needed, leading to a series of studies into the fundamentals of physics simulation for thin, fibre-like elements.

\section{Research Partners}

The research benefited greatly from both academic and industry partners. Within the University of Stuttgart, the collaborative work on coreless winding at The Institu of Building Structures and Structural Design (ITKE) was performed in partnership with colleagues from the Institute for Computational Design and Construction, led by Professor Achim Menges. The Innochain network brought in three industry partners over the course of the project: Foster + Partners provided the viewpoint of a possible end-user; S-Form provided initial guidance on materials and typical composite-processing techniques: and FibR provided an industrial partner for the fabrication f coreless-wound parts (in collaboration with which the o Innochain prototypes were produced).

\section{Research Outcomes and Applications}

Two large-scale filament-wound installations were produced in collaboration with other research projects, alongside two research-specific prototypes. The Elytra Filament Pavilion, a $200 \mathrm{~m}^{2}$ composite canopy, was installed in the V\&A Museum, London, in May 2016 (Koslowski et al., 2017); and the ICD/ITKE Research Pavilion 2016/2017 (Solly et al., 2019), a 12m-long composite cantilever, was installed on the University of Stuttgart campus in April 2017.

The Fibre Beam Prototype, a 3.6m-long composite beam, was created to test layout optimisation strategies for a fibre-wound beam, and was exhibited at the Innochain Barcelona Colloquium in 2018. The Fibre Façade Prototype, an installation consisting of eight unique glazing-support frames, wound on a single reconfigurable form, tested several digital design tools created during the research, and was exhibited at the final Innochain exhibition in August 2018.

Through the creation of these physical pieces, a prototype fibre-winding toolchain was developed to support the steps from concept to fabrication. Most of the investigations were made into fibre-winding physics simulation, and this work is being continued to produce a digital design tool for future projects.

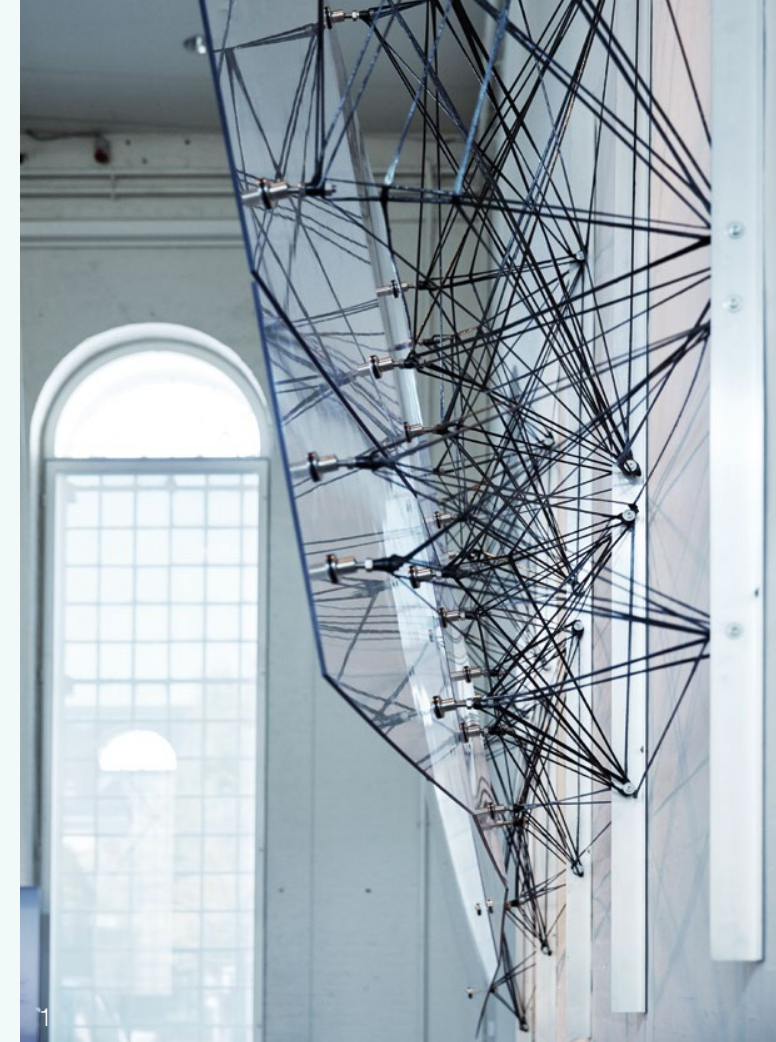

1. Fibre façade prototype at the Innochain Exhibition, Copenhagen. Photo: Anders

2. Fibre beam prototype created for the Innochain
ITKEEFFibR.
Bibliograp

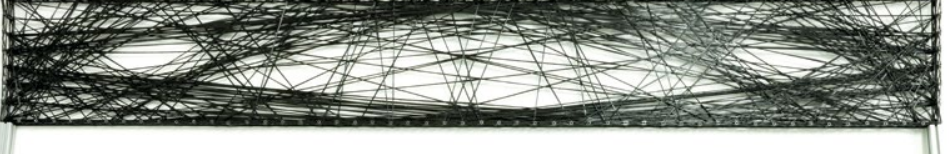

Schwinn, T., La Magna, R., Reichert, S.,
Waimer, F., Knippers, J., Menges, A., 2013 'Prototyping Biomimetic Structure for Architecture: The Confure Architecture: The Conference Papers,
London, Building Centre Trust, p. $224-244$. Solly, J., Früh, N., Saffarian, S., Aldinger, L., Design of a Lattice Composite Cantilever' in Structures, Vol. 18, Amsterdam, Elsevier

Koslowski, V., Solly, J. and Knippers, J., 2017 Structural Design Methods of Componen Based Lattice Composites for the Elytra (eds.), Proceedings of the IASS Annual Symposium 2017, Interfaces: architecture. engineering.science, Hamburg, HafenCity

\begin{abstract}
as material solidification is required during fabrication, Coreless Filament Winding (CFW) provides a high-speed alternative. In this approach, uncured fibre bundles are sequentially wrapped around reusable, reconfigurable frames, which are then cured. Invented at the University of Stuttgart (Schwinn, et al., 2013), the method is under continual development In addition, through several buit projects, a user-friendly set of winding-simulation tools to assist the design process and enable wider adoption of the method have been developed.

Research Methods

As the project was embedded within a wider ongoing research area at the University of Stuttgart, the research methods employed were informed by a three-year plan involving the development of two large-scale installations fabricated by means of the CFW method. The first majo phase of work comprised of a state-of-the-art review, in which research question-formation was delivered through a research-by-fabrication process. Physically realising a project alongside experienced researchers in the field provided access to significant backgroun knowledge on the fabrication system. Furthermore, involvement in the design development and engineering of these projects gave direct experience of specific CFW design problems and the opportunity to consider which nes could be solved using virtual prototyping tools.
\end{abstract}




\section{Further Perspectives \\ Data in Design Practice}

Sean Hanna

The Bartlett School of Architecture

For many of the projects discussed in this volume, the most fundamental unit of their design has begun to shift from material or representation to data, and so our emphasis on information and transaction has become increasingly relevant. Architects need data, as our design problems reach unprecedented scales and complexities. As more varieties of data are made available, in exponentially increasing quantities, new and improved methods must be sought to visualise, understand and make adequate use of it. The methods of data processing appropriate to other domains may not be appropriate to architecture and concern is shiftin from the practical and technical problems of how data is processed to the more complex issue of how it fits in with design practice.

Optimisation is a traditional and well-understood use of data, in which a given design is incrementally improved toward a specific soal, and an explicit, quantifiable definition of a problem is essential It is often given by a parametric model, which expresses the design outco para function of specific numerical inputs, and many as a function of spectic numprical ine de and many of the dominat trends in archica design. Design is not all optimisation, however, a design. Design is notalloptimisation, however, and much of the discussion surounding the work at the has focused on the ple data plays in the less clerlyhas focused on the role data plays in the less clearlydefined aspects of design practice: early-stage design

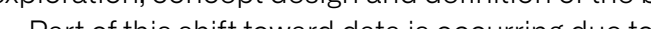
echnological advances, particularly faster computation speed and the algorithmic improvement that accompanies it. Computational fluid dynamics (CFD) methods, such as those developed by Angelos Chronis igs 1 \& 2; see also p.76-77 of this volume), yield faster analyses of design proposals that give the user immediate, real-time feedback on proposed changes. Where analysis was once a distinct activity from the act of design, it is now an integral part. The familiar colourindexed visualisations that represent CFD, structura simulations and similar analyses have long been used by designers to examine relevant data, but the move to make them a part of early-stage sketch modelling and interaction allows one to see phenomena that are otherwise invisible. The novelty of this expanded sense of vision, coupled with the capacity for machine learning and related technologies in processing the output produced by these methods, underscores the need for a more mature understanding of the designer's use of such data. Part of the challenge identified can be understoot in light of the apparent opposition between intuition and explicit quantitative methods. The former is often how designers work but is a black box in terms of our lack of understanding and our inability to communicate intuitive thoughts. The latter, in which most da resides, provides the basis for legal in which ments resp the basis for legal requirements, responsibility and describing the project to others. These domains may divide designers collaborating on a project. One obstacle to overome in cosign practice is the between the higher-levelview or the architect [and] the Cher-levelvew or the expert [n a Part of the chal

Part the challenge is due to the type of data itself, and its limitations. Traditional means of data-processing assume that we are able to understand what the data represents - the physics behind structural calculations or the volume of traffic - even if there is a large amount of data available. In the age of big data, where both the be mined with pattern recognition and machine learning

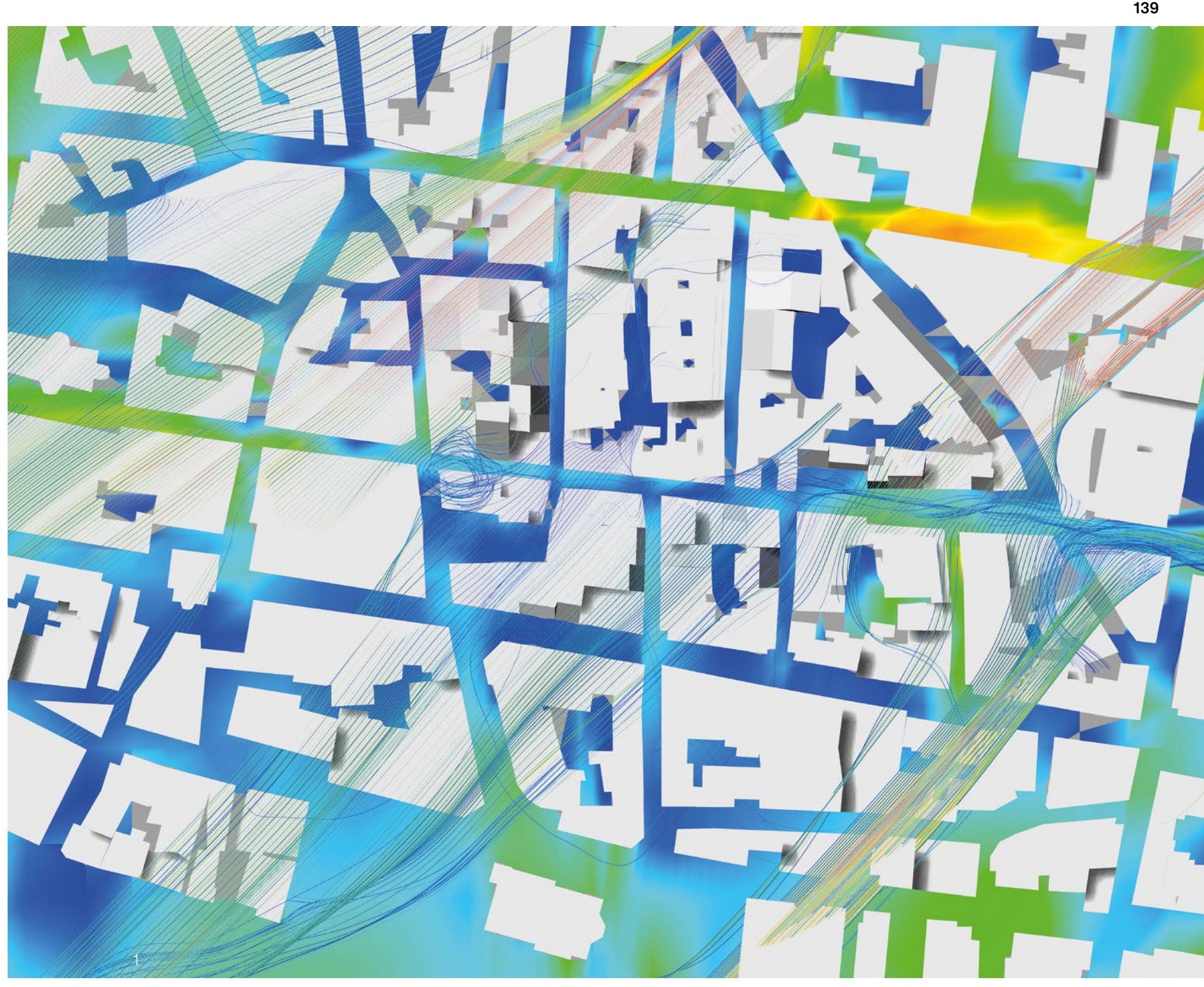

algorithms to be useful, the designer may not be able to understand the meaning of the numbers, even in principle. This raises a new kind of question: 'Do we need to?' 'Does the neural network need to?'

Zeynep Aksöz's research on the Innochain project (summarised in p.50-55 and p.74 of this volume), which incorporated machine learing into structural optimisation and multi-criteria searches, illustrutes that there are at least two differentstrategies for humancore are least two diferent strategles for humancomputer interaction. The first, in the context of a structural bullang façade project, uses the designer's own preferences as the data, collected through a process in which the user selects preferred solutions from generated examples based on their own intuition or aesthetic judgement. A support vector machine 


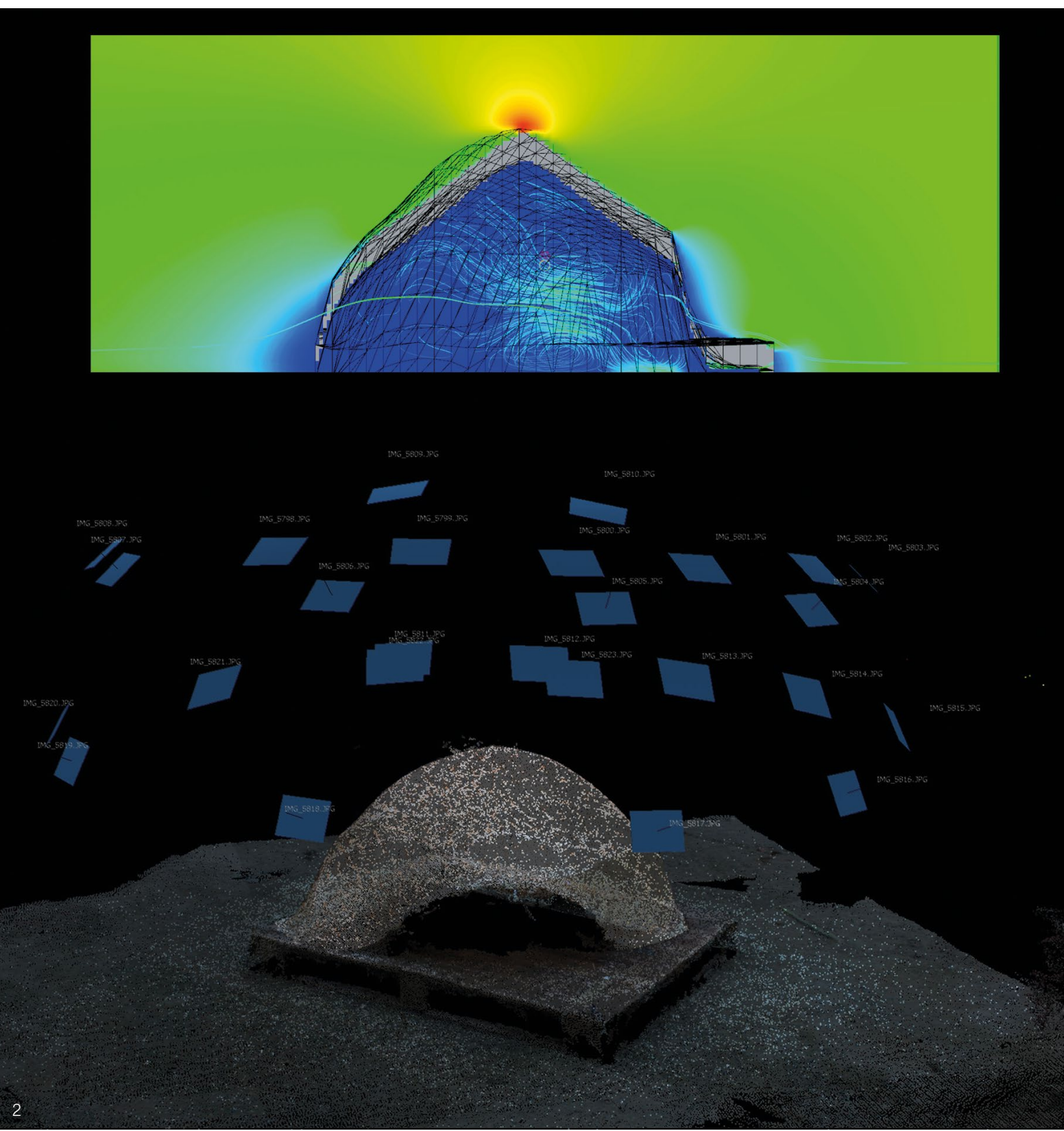

2. Simulation and analysis in the CAD mode can be compared with as-built scan

3. CityFunction software, Henrik Malm and
Petra Jenning FOIAB Architects.

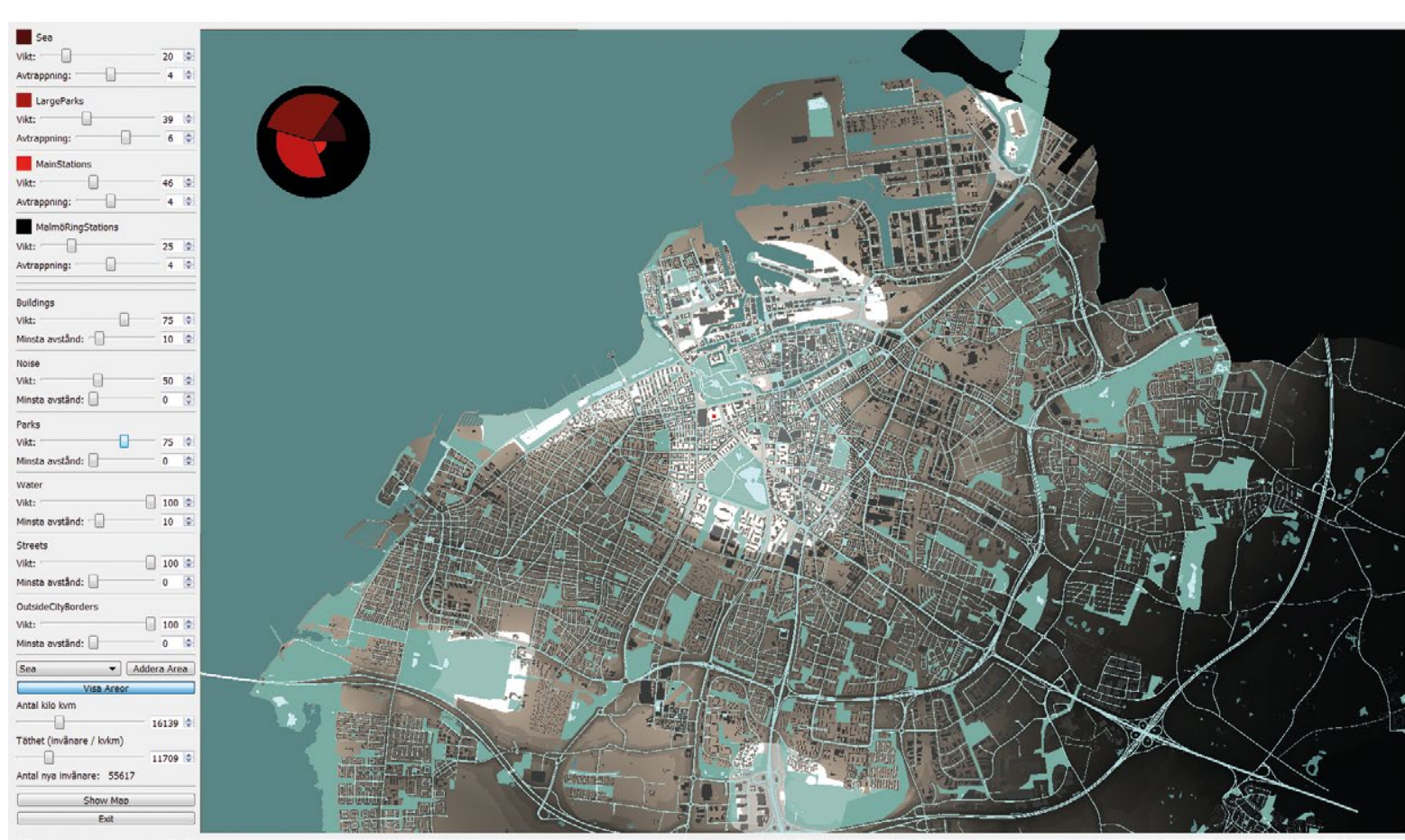

the designer in further optimisation. A contrasting approach has the designer maintain global control over the project, while the computer makes only loca corrections to improve the structural geometry. Both approaches involve collaborative efforts between use and computer, and their use in practice suggests that, at least for early-stage exploration of design options, the architect's overall sense of the project at the highest level is what is valued most. The architect wants a clear picture that can be provided by the machine, and clear picture that can be provided by the machine, and only In some projects, the obstacle is the limit of the designer's high-level intuition and data is used to devercome this. Urban planning is a good used to of large-scale complexity: the paradigmatic 'wicked problem' Henrik Malm and Petra Jenning of FOAAB problem. Hen visualis tion tool that directly displays the geographica visualisation tool hat diectly displays the geographical distibution of imm andity, time-sensitive sensor data, such as pollution and traftic, and second-ordercalculations, such as distance to parks and commuting the. Because these sources are numerous, the City Fiction tool presents a combination of raw data in the form of a user-de

connotations for optimisation, the concerns that have been raised in projects of this scale have less to do with finding an optimum solution than with our level of certainty that the data are stable over long timespans. By visually presenting patterns otherwise spread too far across space or time to be seen, the expert pattern-recognition skills of the designer's proposed on the back of such a tool might be of the kind equipped to deal with the city of 2050 , its real value being the ability to define different fitness functions, to test multiple scenarios for sensitivity to change and roustnes, and so to allow us to comprehend a range of possibilities.

If there is a tentative consensus on the use of data lesign practice across the range of projects and methods currently in use, it is that the levels of a proiect must be handled individually; there is no clear rou to optimise a project to a given dataset. In most cases, it is ackno project to a given dataset. In most cases, to ack with the globat direction of a project, whilen comp is well suted to hand of computeris well sulted to handing pe details, particularly Wive project will use a strategy incorporatis muthiple given project willuse astrategy incorporating multiple layers of cha, each a forming the design wa diferent methods and, ultimately, combering themeasurable brain may effectively be brought to bear. The strategies 

Rewired Engineering:

The Impact of Customisation and Interoperability on Design

Edoardo Tibuzzi

AKT II, London

Once upon a time the conquering of physical or territorial realm was the new frontier. But to conquer sordid, ugly commercialism in this machine age ... this conquest is now 'the new Frontier'. Only by growing a healthy aesthetic in the Soul of our polyglot people can we win this victory.

Frank Lloyd Wright, When Democracy Builds

Introduction

The aim of this text is to look under the hood of a practice that has, in one way or another, pioneered the digital transformation of the architecture, engineering and construction (AEC) industry, and to explore how collaboration and use of advanced modelling and analysis tools have changed the design outputs. It also explores the important role of research in practice and academi The digital revolution has had a huge impact on most aspects of our society, and in recent years, it has also begun to invest in the AEC industry. This revolution has brought two main outcomes: a digitalisation of the has various aspects of design and analysing comparing and finding a pects 20 -odd years ago in the eary cays of AKT II structur engine (sill called Adams Kar Taylor back then), engineers (still called Adams Kara Taylor back hen), the digital revolution was just begining. Our vision, as structural engineers, was clear. to establish a group able to pioneer change and inestigate the potental impact of the articial, digital 'machine', and so, p.art was created. The singular remit of this cross-disciplinary group - which consisted of engineers, architects, computer scientists and parametric designers was, and stil is, to explore and capitalise on new opportunities via technological and software
development, through in-depth research into new

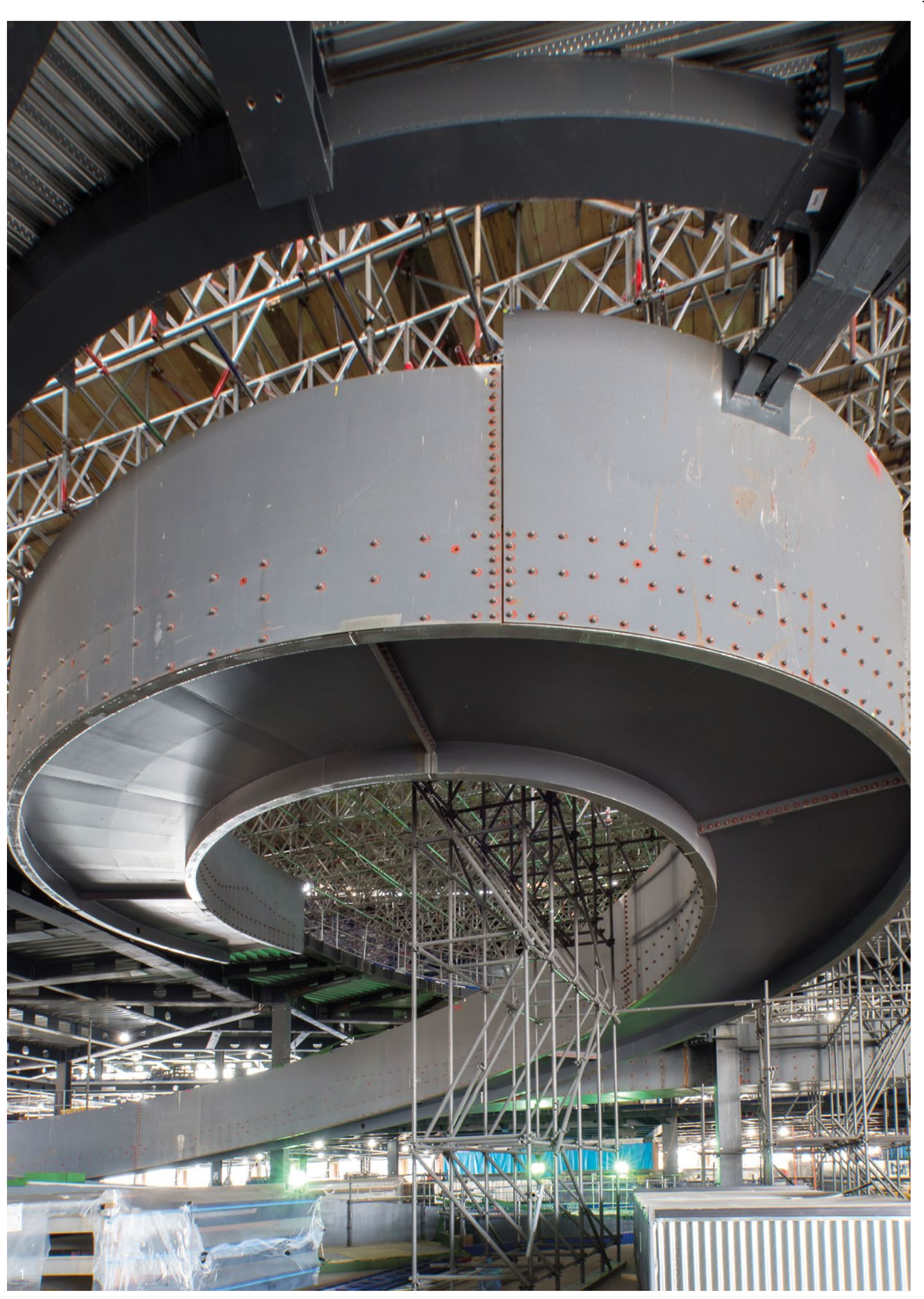




\section{Bloomberg Headquarters, London}

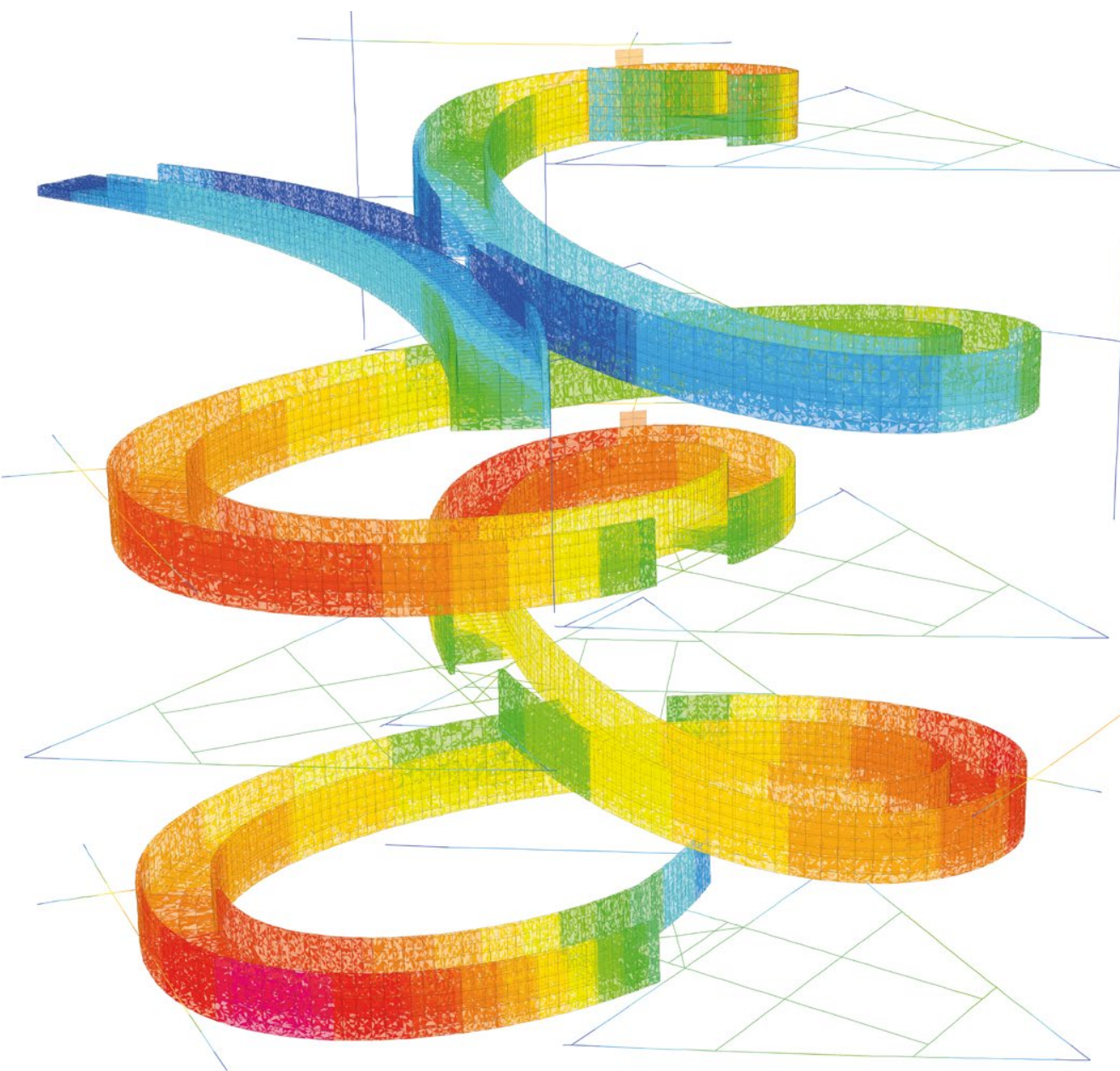

materials, construction and fabrication techniques, as well as new digital tool sets and software interfaces that improve coordination between the different partners in design and construction teams.

The technology that we were using at the beginning generated, implicitly or explicitly, all sorts of forms

through democratic parameters. The first generation of digital design was born and 'parametric design' was the dighe used to describe it Our focus then intensified looking in to how to join up the structurat engineers looking into 'aw in join up ' This res of 'taming gravity' with digital fabrication. This resuled h This resuled in interoperabilty probems beingeering and contrict constructor. The problem of enabling interoperability differgh a soltware interface is still with us today, the only diference being the brute force of computation that has given us even more ways to break it down. Interesting the problem was not to solve collaboration (real or imaginary) with software, but to enable it with caution, the danger being that collaborative design could produce banal and trivial outputs.
We had the privilege to collaborate with Foster + Partners on the design of Bloomberg's new European headquarters. Set within the heart of the main office building, there is a unique bronze-clad stepped ramp, a continuous $3 \mathrm{D}$ loop that is as much a piece of art as it is a key aspect of movement through the building. All arrivals are taken by lift to the 'Pantry' - a double-height entrance space on the sixth floor - from which they use the ramp to filter down to their floors. The ramp has been designed and proportioned as a place of meeting and connection, allowing people to have impromptu conversations with colleagues, while not impeding the flow of people. On plan, the ramp is hypotrochoidal, meaning that it follows a line traced by a fixed point within a circle, as the circle rotates within another circle. Rising from level two to eight, the ramp is $1.8 \mathrm{~m}$ wide between balustrades and spans up to $30 \mathrm{~m}$ between floors; the elliptical oculus within the surrounding floor plates rotates $120^{\circ}$ on each floor to follow the curve of the ramp and form connections. Constructed as a structural monocoque, where the chassis is integral with the body, rather like ship's hull or a bridge, the ramp was prefabricated in large interlocking segments, staggered and bolted as a kit of parts, but ultimately forming a smooth continuous structure. The floor at level seven is cut out to create the double-height Pantry volume which sits between levels six and eight; this means that the ramp is not connected to the floor plate here as it is on other levels. Access was resolved by inserting a sloping link-bridge that connects to the ramp and floor edge. Both ramp and link-bridge are structurally coupled and act as twin structures supported by two of the main central columns that terminate at this interface. The structural performance or the ramp was assessed for a series of combinations of design parameters.

At the design stage, Foster + Partners and AKT II At he deign stage, Foster + Parthers and AKTII The whol design was controlled through a singul. Thewrithm, in algorthm, interfacing which allow for an and simulation packe assessment of the valious forces driving the geometry limit, we needed a more in - depth and this of the imal limit, we needed a more pus de of human-induced vibration. The analysis results were launched and retrieved from the same algorithm, allowing us to inform the architectural design and shape the ramp to improve problematic areas or to save material, where possible.

Design is largely collaborative, and, without a clear definition of targets, it can result in homogenisation of an output where all disciplines are equal. From parametric design of the early stages to post-digital and beyond, we are asking ourselves: 'Where are we now? and 'What
Systems found in the natural world can perform computations up to a maximal ('universal') level of computational power, and most systems do, in fact attain this maximal level of computational power. Consequently, most systems are computationally equivalent. For example, the workings of the human brain or the evolution of weather systems can, in principle, compute the same things as a compute. Computation is therefore simply a question of translating inputs and outputs from one system to another (Wolfram, 2002).

Essentially, whenever one sees behaviour that is not obviously simple, in any system, it can be thought of as corresponding to a computation of equivalent sophistication.

Swedish philosopher Nick Bostrom argues that at least one of the following propositions is true: 1. The human species is very likely to become extinc before reaching a 'posthuman' stage; 2 . Any posthuman civilisation is extremely unlikely to run a significant number of simulations of its evolutionary history (or variations thereof); 3 . We are almost certainly living in a computer simulation. It follows that the belief that there is a significant chance that we shall one day become posthumans who run ancestorsimulations is false, unless we are currently living in a simulation (Bostrom, 2003).

The two theories from Wolfram and Bostrom are reaffirming the role and power of computational design in both the real and the simulated realms, but it is important to remember that a conflict remains between aesthetic, structural and technological efficiency and value. So, for a practice like us, it is imperative to acknowledge that technology is driving us to a position that is binary. Without soul tools only give a sterile answer, and if the question is wrong, so too will be the answer.

The thought that technology as truth absolves mora The tibility is also dancorous. It removes the need for agency and accountability. and most of all, puts creativity in danger of extinction. Tools are not innocent, and pervasive analytics can create dysfunctionality as people act on such insights because they are beatifully presented, but they can be entiely mish arpeautifyly present but her presenter design stages, to bidge digital tols allowing our design stages, to bridge distal tools, allowing our end This interface was used in the following project.

\section{The Serpentine Pavilion, London}

For The Serpentine Galleries' annual event in London's Kensington Gardens, we provided structural engineerin services for the design of the superstructure of BIG's 2016 Pavilion, envisioned as an 'unzipped' Wall or glass- 


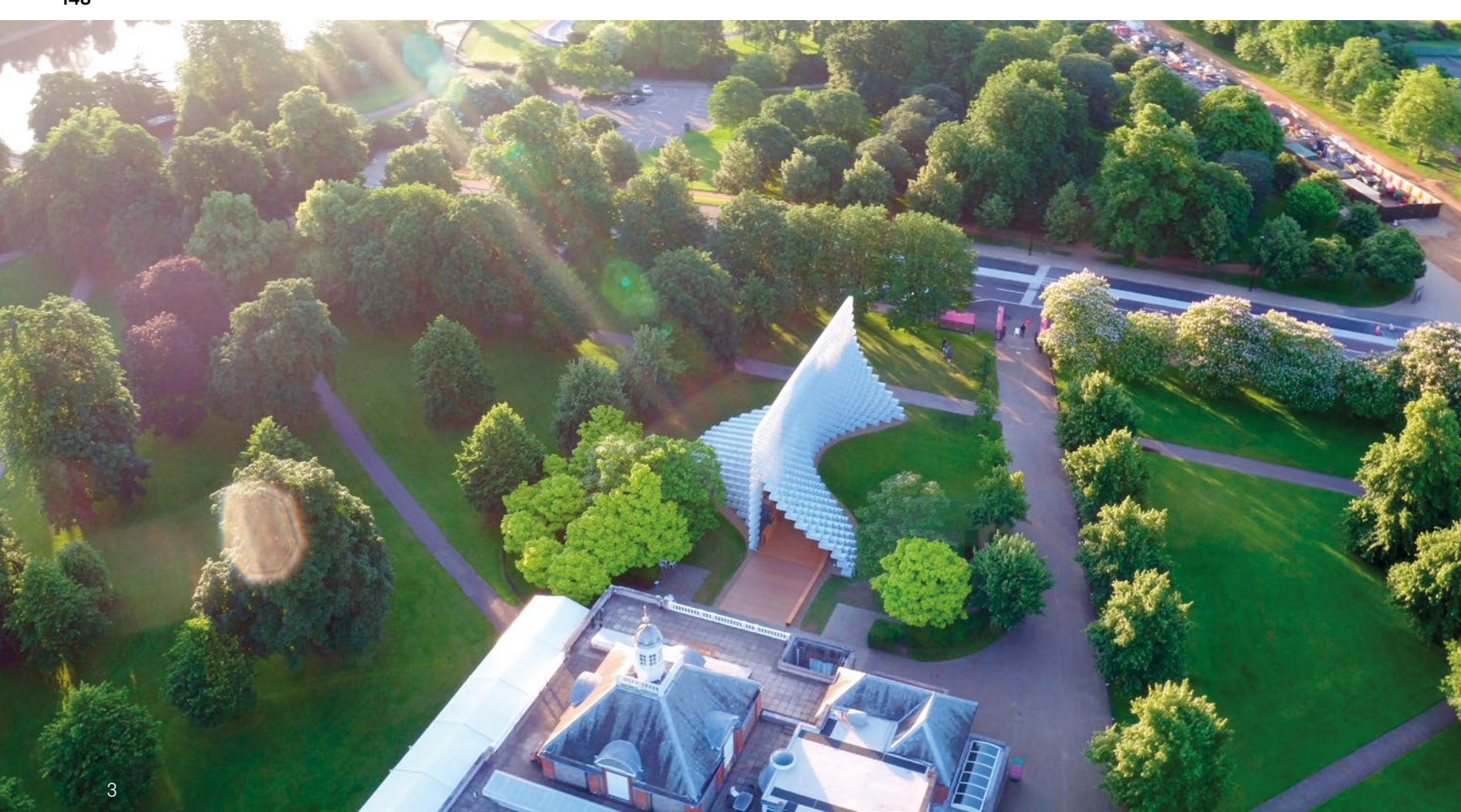

comprised two surfaces, which began as separate curved sinusoidal walls at ground level and rose to merge than three months. In addition to these time pressures, budgetary constraints required material quantities to as a straight horizols line at an eve an $14 \mathrm{~m}$ above be reduced, as far as possible, without compromising ground. The surfaces were formed from a series of the ambition of the design. For these reasons, the BIG and AKT II design teams chose to boxes, each measuring $500 \mathrm{~mm} \times 400 \mathrm{~mm}$. The length of and each box was such that they overlapped their neighbours geom sufficiently to create an enclosure, while there was also enabled the rapidid for structural purposes. The transition from two separate the undenlying sid sarare grids at differents halves to a single surface at the apex was achieved by as well as more complicated pin-wheel and reciproca arranging the boxes in alternate chequerboard patterns arrangements for the boxes. For whe antion, the design arranging the boxes in alternate chequerboard palterns arrangements for ho boxes. For each option, the design on either searlo roved mixture to enhance the materials ingitis these were then joined by aluminium con igdt the arching action of the pavilon were transferred between the bicks by pun -pull forces in the connecting bolts, along he overlapping edges of the boxes. In the longludinal direction, horizontal wind loads were transferred through a combination of shell and Vierendeel action. The boxes were stepped such that people could sit or climb on the lowest rows, which was considered in the loading. To realise such a large and structurally complex building, it was necessary to go from concept design fully coordinated production information in less values such as the individul box hight and with to macro diment between adjacent boxes, overall wall heights, length between adjacent boxes, overall wall heights, lengths and sine wave proportions - and interrogate the resultin for fixings and so onant At every foterial volume, number of fixings and so on. At every iteration, these metrics were passed ar inconerion In conclusion, our industry is preparing to face an important shift in the controlling forces of design, where data will play a fundamental role in rewriting codified approaches and performance-based choices. New roles and skills will, therefore, become fundamental in the design process to maintain a balance between optimal and functional, optimised and harmonic,

4
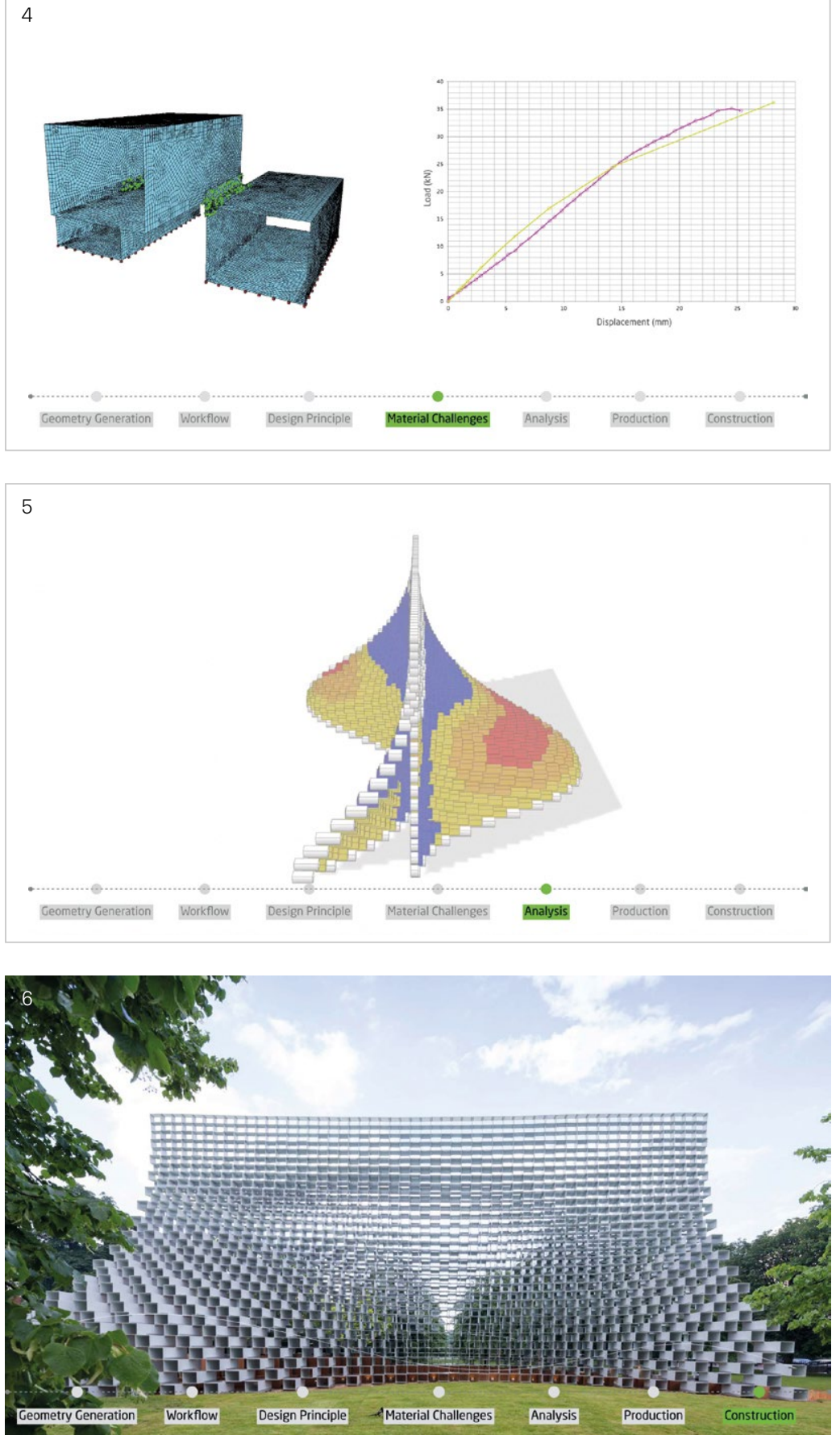

3. Drone photo of completed BIG
Serpentine Pavilion. O AKTII.

4. Load/deformation comparison between
FEA Model and Physical test. () AKTII.

5. Visualisation of forces acting on box

6. Front photo of completed pavillion. ๑ AKTI.
Bibliography

Bostrom, N., 2003, 'Are You Living in a Computer Simulation?' in Philosophical
Quarterly, Vol. 53 , No. 211, p.243-255.

Kara, H. and Bosia, D., 2016, Design

Engineering Refocused, London, John Wiley

Theman The 2016 Serpentine Pavilion. A case Stud in Large-Scale GFRP Structural Design and and Skavara, M. (eds.). Fabricate Rethinking Design and Construction,
London, UCL Press, p.138-145.

Rabagliati, J., Janssen, J., Tibuzzi, E., De Paoli, Rabagliati, J.,Janssen, J., Tibuzzi, E., De Paoli,
F., Casson, P. and Maddock, R., 2018,
Bloombers Ramo Codllaborative Workflows 'Bloomberg Ramp: Collaborative Workflows,
Sharing Data and Design Logics' in De Rycke, K., Gengnagel, C., Baverel, O., Burry, Thomsen, M.R. (eds.), Humanizing Digital Reality, Design Modelling Symposium Paris Pte Ltd., p.153-166.

Wolfram, S., 2002, A New Kind of Science, Wright, FL., 1945, When Democracy Builds,
Chicago, University of Chicago Press. 


\section{Drone Spraying on Light}

\section{Formwork for Mud Shells}

\section{Stephanie Chaltiel}

MuDD Architects

Maite Bravo

BCIT Vancouver

Diederik Veenendaal

Summum Engineering

Gavin Sayers

AKT II

\section{Introduction}

Recent developments in robotic fabrication have led to the rapid emergence of novel methods of additive manufacturing, using either extrusion or spraying of wet and dry concrete mixes, also known as 'shotcrete' which has a long history (US Army Corps, 1993) and is widely. used today in diverse construction applications, with variety of formworks.

'Bioshotcrete' refers to experimental construction techniques that combine additive manufacturing with mortars made of natural materials instead of concrete, facilitating the construction of architectural envelopes and vaulted structures referred-to as mud shells. These techniques involve the drone spraying of raw clay, sand and fibre mixes (i.e. 'biomortars') and are currently under development by a multidisciplinary team of engineers and architects. A drone is fitted with a hose that sprays successive deposition of biomortars over light formwork (Bravo and Chaltiel, 2017).

Some of the many advantages of using bioshotcrete include: avoiding the use of heavy machinery onsite; reducing the nocity reducing the necessity for labour intensine bespoke In this text a brif history of the emergence In this text, a bief history of the energence and development of bioshotcrete is presented. Two case reques a for diferst types of light formu fabication from the reful formu the careful formulation of biomortars and their correct deposition sequencing. Finally, possible future scenarios for the implementation of this technique are outlined.

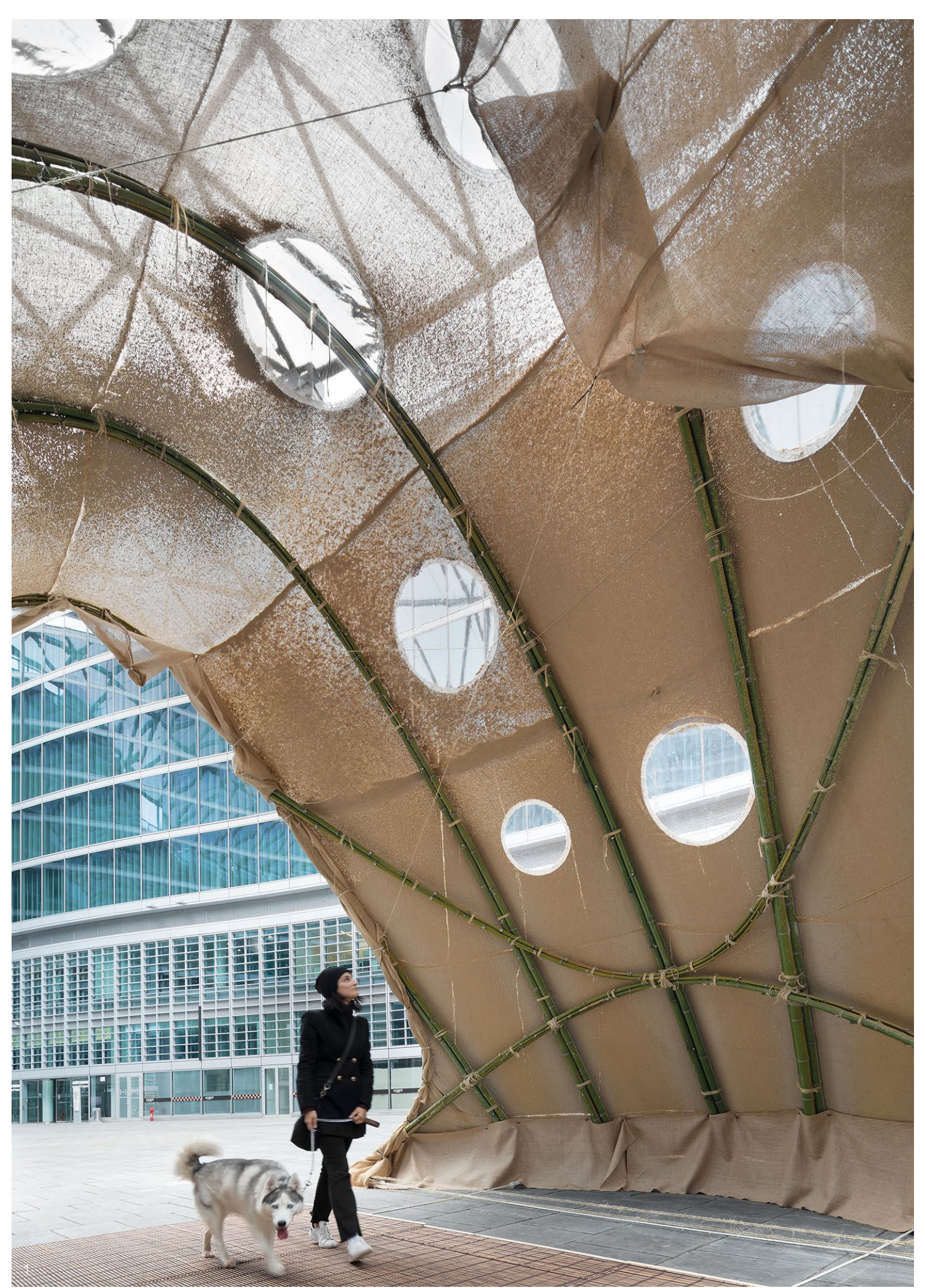


The proposed technique for the fabrication of monolithic earthen shells with drone spraying is based on a precise protocol.

The process starts with material formulation preparation and sequencing (Bravo and Chaltiel, 2017) including the selection of suitable material mixes. The material has been under development since 2012, in
collaboration with the CRAterre laboratory and Wilfred Carazas, a leading expert in raw earth construction. For drone depositions, ongoing experiments started in December 2017 conducted with RcTakeOff, which developed a bespoke drone for bioshotcrete. These experiments yielded an improved system whereby the drone was fitted with an Euromair hose, connected to a powerful pump on the ground.

\section{Light Formwork}

Several types of lightweight formworks, suitable for drone spraying, have been explored in built projects since 2018: - an inflatable formwork for a dome, inspired by the Bini-dome system and other pneumatic formworks (Van Hennik and Houtman, 2008),

- a geodesic frame with dry jute bags attached, inspired by the Concrete Canvas product (Chen et al., 2016), and

- a set of bamboo arches with fabric stretched across, inspired by the Ctesiphon system by James Waller and other fabric formworks (Veenendaal, 2016).

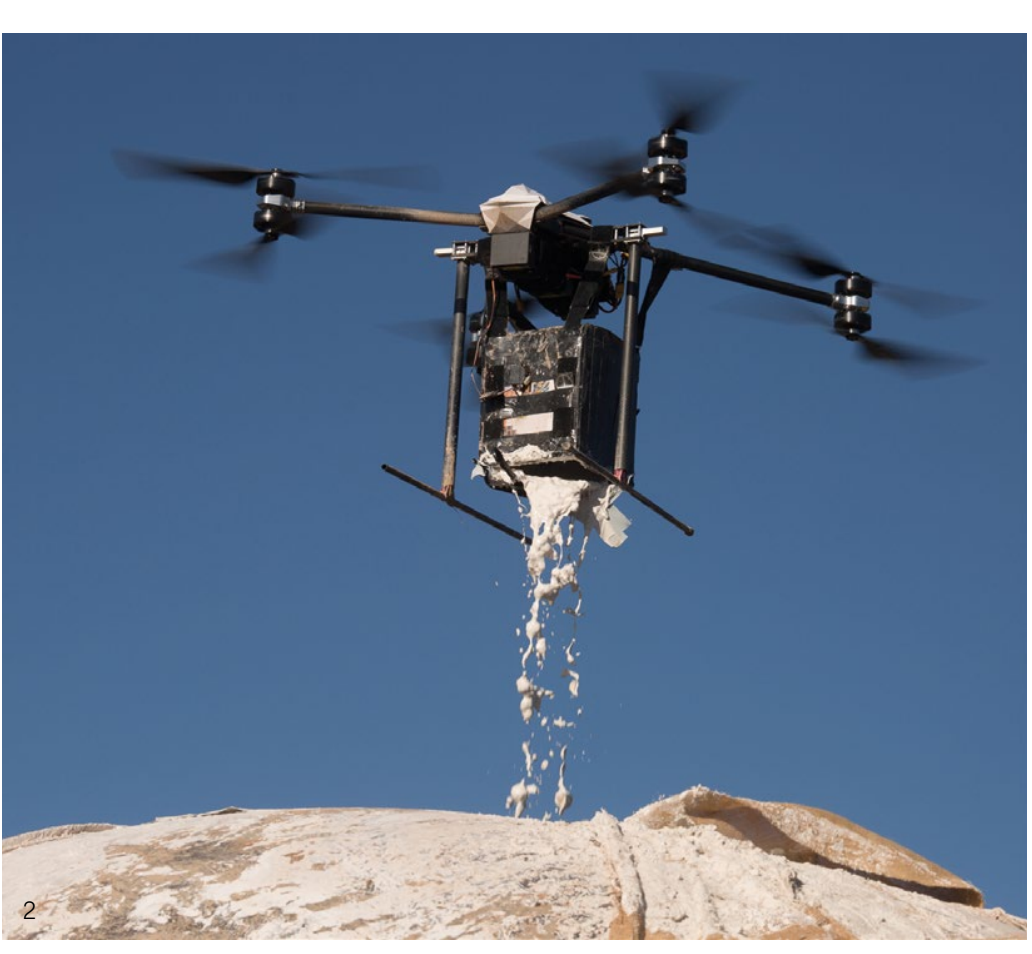

2. Drone depositing a coat over a large inflatable dome. Barcelona Drone Ce
2018. Photo: Frederic Carmona.

3. Mud shell geodesic design where the entrance is reinforced with two poles. The
five top triangles of the geodesic dome are made of plastic sheets to protect the
Titilit tests at the Barcelona Drone Center explored the possibility of using a drone to undertake several trips to coat a large inflatable dome (fig.2), but encountered challenges in terms of process-related inaccuracies final structure (Šamec et al., 2018) and, especially, stability (Bravo and Chaltiel, 2018).

To address these issues, two possible solutions wer

Case Studies

Two case studies implemented distinctive techniques allowing fast, onsite formwork assembly and an easy

. diameter. It investigated drone spraying with various laye secured onto a geodesic frame. Terramia, the second case study, is composed of three vaulted structures spraying of locally sourced biomortars with different Mud Shell London Design Festival 2018 The Mud Shell project by MuDD Architects was built for Anthe South Bank, identied for formwork. a prefabricated geodesic wood

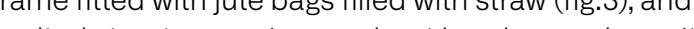
stretched fabric (figs. $1 \& 6$ ). of biomortars on dry, prefabricated insulation modules

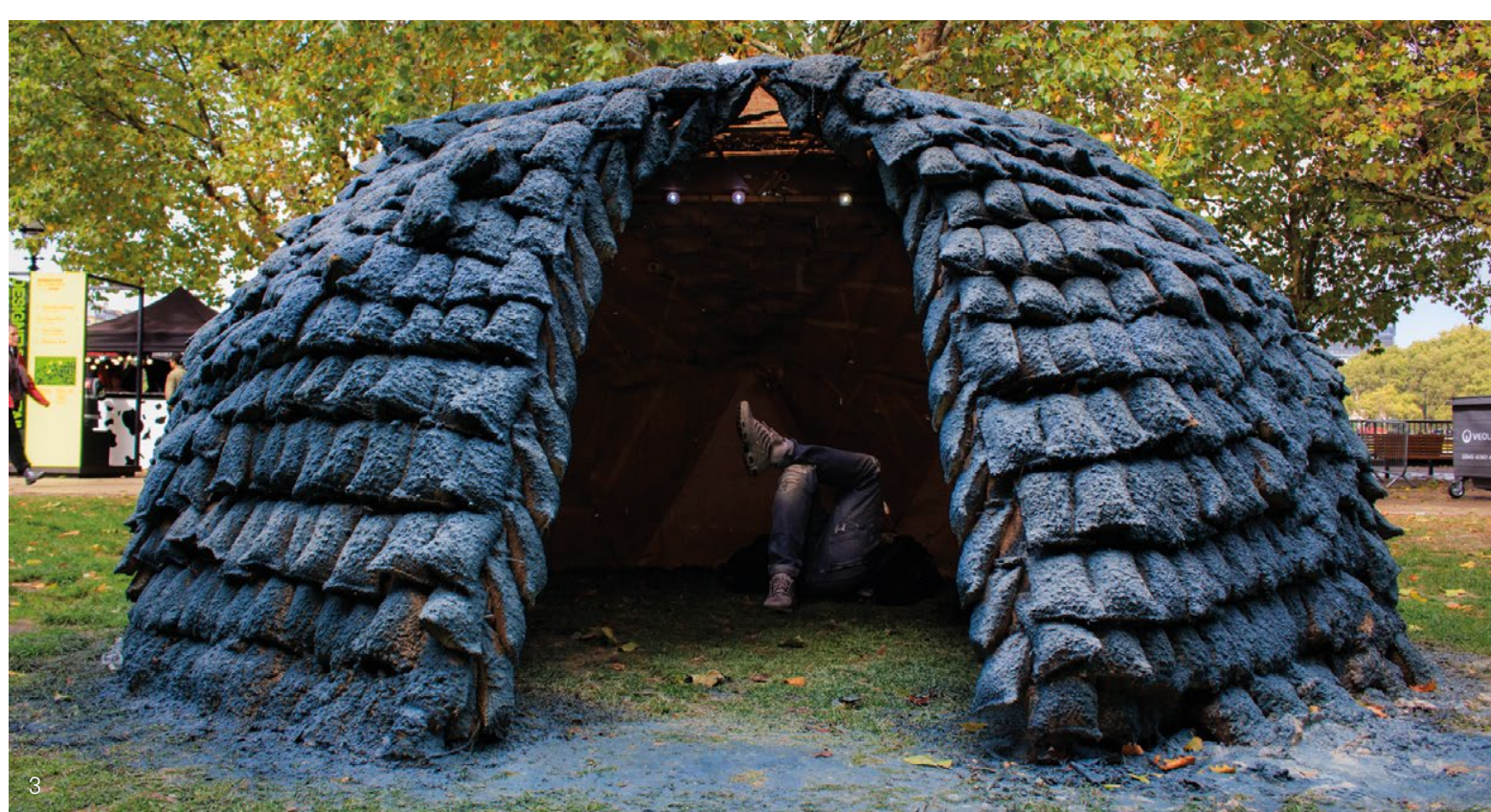

where the drone spray sessions were organised as live demonstrations for the public.

The construction process started with the assembly of a pre-made geodesic wooden frame composed of 35 triangles with 3D-printed PLA connectors complete with locking caps fitted on the inner side for reinforcement. Some 1,750 prefabricated, light-insulating, $15 \mathrm{~cm} \times 20 \mathrm{~cm}$ jute fabric modules were secured to act as lost formwork, filled with $3 \mathrm{~cm}$ straws positioned in rows with a $5 \mathrm{~cm}$ minimum overlap.

The spray phase featured a drone, fitted with a $20 \mathrm{~m}$ hose connected to a Euromair pump, which allowed the pilot and co-pilot to coat the structure in a uniform way without repositioning any of the machines or tools, as hose was flexible enough to turn around the structus The construction process was undertaken within a meshed space so that itcould be classified as an indoor project which only requires health and safety approval project which only requires heath an safety approval The drow therefor spraying inside aresticted are The dronewas, th which 70 desrees, so as to coat the entires of between 0 dome. The -amp Euromair Pumpalowed the war dome. The 40 -amp Euromair Pump allowed the water hom to be ecctonically inut, providing a consistently homogeneous mortar and material grains not exceeding Imm. A 5mm-diameter nozzle was used for the first layer, and a $1 \mathrm{~cm}$-diameter nozzle for the final layers, at Huctuating distances of between $10 \mathrm{~cm}$ and $100 \mathrm{~cm}$ from the surface. The speeds of deposition were between $50 \mathrm{~cm}$ and $100 \mathrm{~cm}$ per second, allowing a flow of matter of 8 litres per second. The materials used for the mort spray included Claytec earth and linen-fibre ready- depositions - six layers of $1 \mathrm{~cm}$ - completed the process. Due to windy and wet weather conditions, the drying me in-between each drone spray session was about three hours. Stirring time, drone speed and type of ozzle were all crucia fo

The shell was based on an earlier built prototype of similar characteristics and size that the same fabrication team built at the Domaine de Boisbuchet in August 2018. This helped to refine the design to avoid damage from rain exposure by covering the top five isosceles triangles with waterproofed transparent sheets and by reinforcin the entry with two inclined poles, defining a smaller open area (fig.3).

The prefabricated dry modules (1m side triangular frames fitted with 50 jute bags filled with straw for insulation) proved efficient in terms of acoustics and wind protection and allowed a fast assembly onsite. The filling of each jute bag by hand proved to be labourintensive, however, and they would need to be produced in an industrial manner if implemented at a larger scale.

\section{Terramia Milan Design Week, 2019}

Terramia was designed and built by MuDD Architects with Summum engineering and AKT II in collaboration with CanyaViva, at the Regione Lombardia government headquarters.

This project proposes the construction of vaulted tructures with lightweight, stay-in-place formwork composed of bamboo arches and a tailored fabric surface, which is later drone-sprayed with clay mortar. (and form-finding sought to rationalise and optimise the competition-winning forms into geometries 


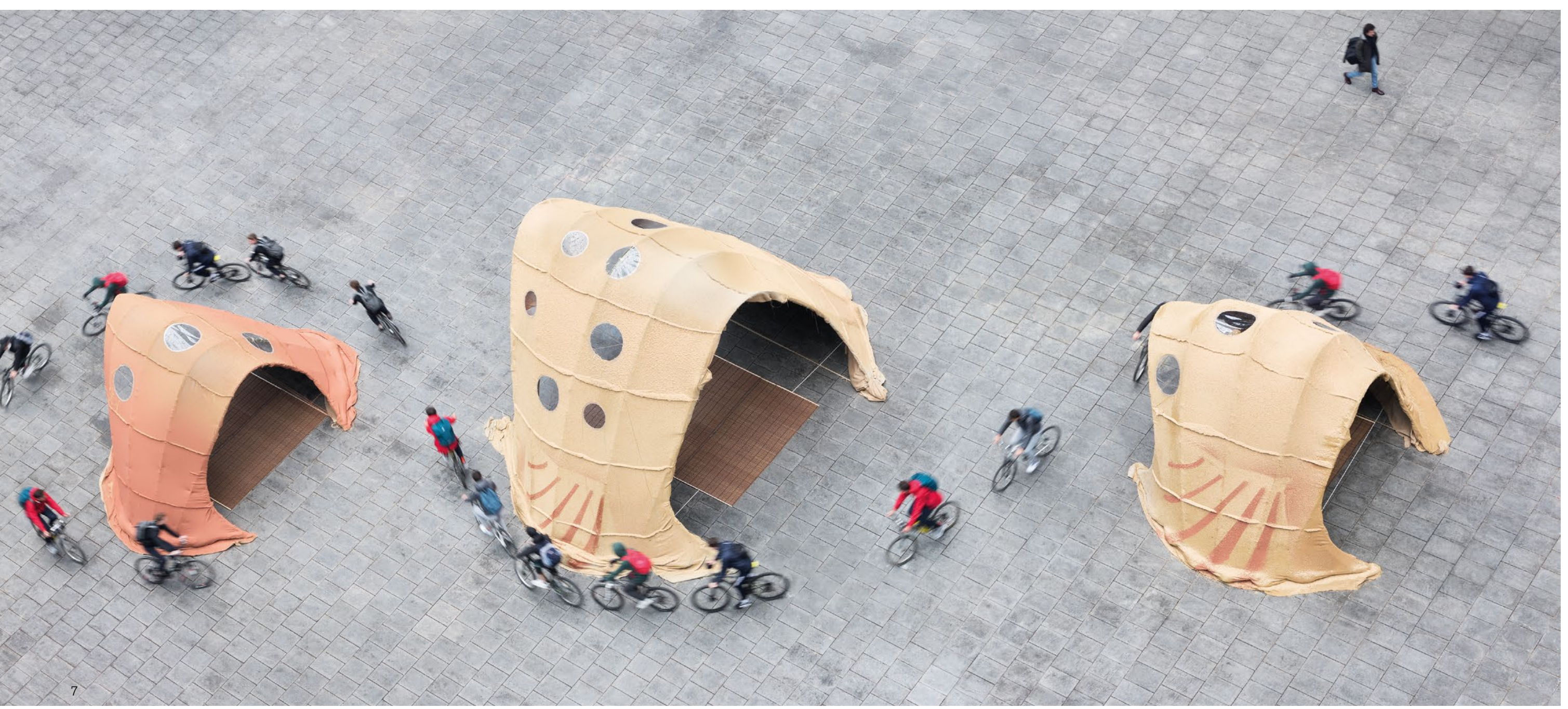

that the drone is fitted with the spraying hose attached that the pump on the ground, but also supplied with electricity by a power cable attanstanty supplied with elctricity by a thered and no tethered along scenarios should be explo

An important project. An important development of the drone-aided construction he authors are proposing for future projects ion the a sach the behind a light mesh for international drone light Tompliance with drone spraying in dense city centres. These large-scale fabrication strategles using locallysourced mortars and light equipment onsite (the drone high-quaty fins of luggage) enable affordable and high-quality finishing of the façade including large-scale ornamentation using a stencil CNC cut to fit the façade such foished.

Bravo, M. and Chaltiel, S., 2017, Monolithic Earthen Shells Digital Fabrication: Hybrid Workflow. The

Bravo, M. and Chaltiel S, 2017 'Paste Matter 3D Printing in Monolithic Shells Fabrication Methods' in Joao de Oliveira, M. and Crespo, F (eds.), Kine[SISIJtem A Architectural Matter June 19th-20th, 2017, Lisbon, to Architectural
Portugal, p.10-18

Bravo, M., Chaltiel, S. and Carazas, W., 2018, Temes de Disseny, No. 34, p.80-91.

Chaltiel, S. and Bravo, M., 2017, DISCIPLINES \& Conference of the Association for Computer Aided Design in Architecture (ACADIA), Cambridge, MA 2-4 November, 2017, p.94-99 'Monolithic Earthen Shells

Chaltiel, S., Bravo, M., Goessens, S., Latteur, P., Mansoun, M. and Ahmad, I., 2018, Dry and Liquid Clay Structural Design' in Proceedinos of International Association for Shell Structures, Form and Force Conference, Boston, July, 2018. https:///dial.uclouvain. February 2020)

Hui, L., Chen, H., Liu, L., Zhang, F., Han, F., Lv, T., Zhang, W. and Yang, Y., 2016, Application Desigh of Concre Geotextiles and Geomembranes, Vol. 44, No. 4. p.557-567.

Kromoser, B. and Huber, P., 2016, 'Pneumatic Formwork Systems in Structural Engineering', in
Advances in Material Science and Engineering, special edition, article ID 4724036.

Šamec, E., Srivastava, A. and Chaltiel, S., 2019, Proceedings of the International Conference on Sustainable Materials, Systems and Structures
(SMSS2019): Challenges in Design and Manasement of Structures (conference paper), Rovinj, Croatia. Tibuzzi, E. and Dayan, M., 2017, Multi-Performative UCL Press, p. 280-285.

US Army Corps of Engineers, 1993, 'Standard Practice Shotcrete Applications, New York, CED Engineerins. Online course documentation. https://www. cedengineering.com/courses/introduction-to-

an Hennik, P. and Houtman, R., 2008، 'Pneumatic Formwork for Irregular Curved Thin Shells' in Onate, B. and Kroplin, H. (eds.), Textiles, Composites and p.99-116.

Veenendaal, D., 2017, ‘Design and Form Finding of Flexibly For
p.105-159. 


\section{Industrialising Concrete \\ 3D Printing: Three Case Studies}

Nadja Gaudillière

XtreeE, Rungis and Laboratoire GSA, École Nationale Supérieure d'Architecture Paris

Justin Dirrenberger

XtreeE, Rungis and Laboratoire PIMM, Arts et Métiers-ParisTech

Romain Duballe

XtreeE, Rungis and Laboratoire Navier, Champs-sur-Marne

Charles Bouyssou, Alban Mallet, Philippe Roux and Mahriz Zaker

XtreeE, Rungis

\section{Introduction}

Experimentation with 3D-printed concrete in architecture has flourished over the past decade and, consequently, so too has the development of industrial-grade manufacturing processes. 3D printing is part of a wave of robotic construction technologies developed with the 'digital turn' in architecture (Carpo, 2012) and, with it, the exploration of the possibilities offered by digital fabrication tools (Gramazio et al., 2015). Robotic technologies for construction sites have been designed since since the mid-20th century, but essentially consisted in the beginning of specialised machines accomplishing a single, repetitive task. Recent research has focused on more versatile machines, such as six-axis robotic arms, to perform a great diversity of tasks. This new approach to robotic construction processes, as well as the 'digital and informational turn' accompanying it makes way for a potentially drastic renewal of the construction industry and its methods. The introduction of these new robotic manufacturin The intraction technologis in th early stages, wh hu her challonges neding to be overcome to reach full h the must be studied inght of the potentialties of concrete 3D printing and other robotic pabication processes, to ensure implementation in the construction industy in

This paper gives a brief analysis of the current advancement of concrete 3D-printing technologies and the challenges facing researchers and companies, alongside three case studies of applications of concrete 3D printing developed by XtreeE. These applications have been developed in partnership with various

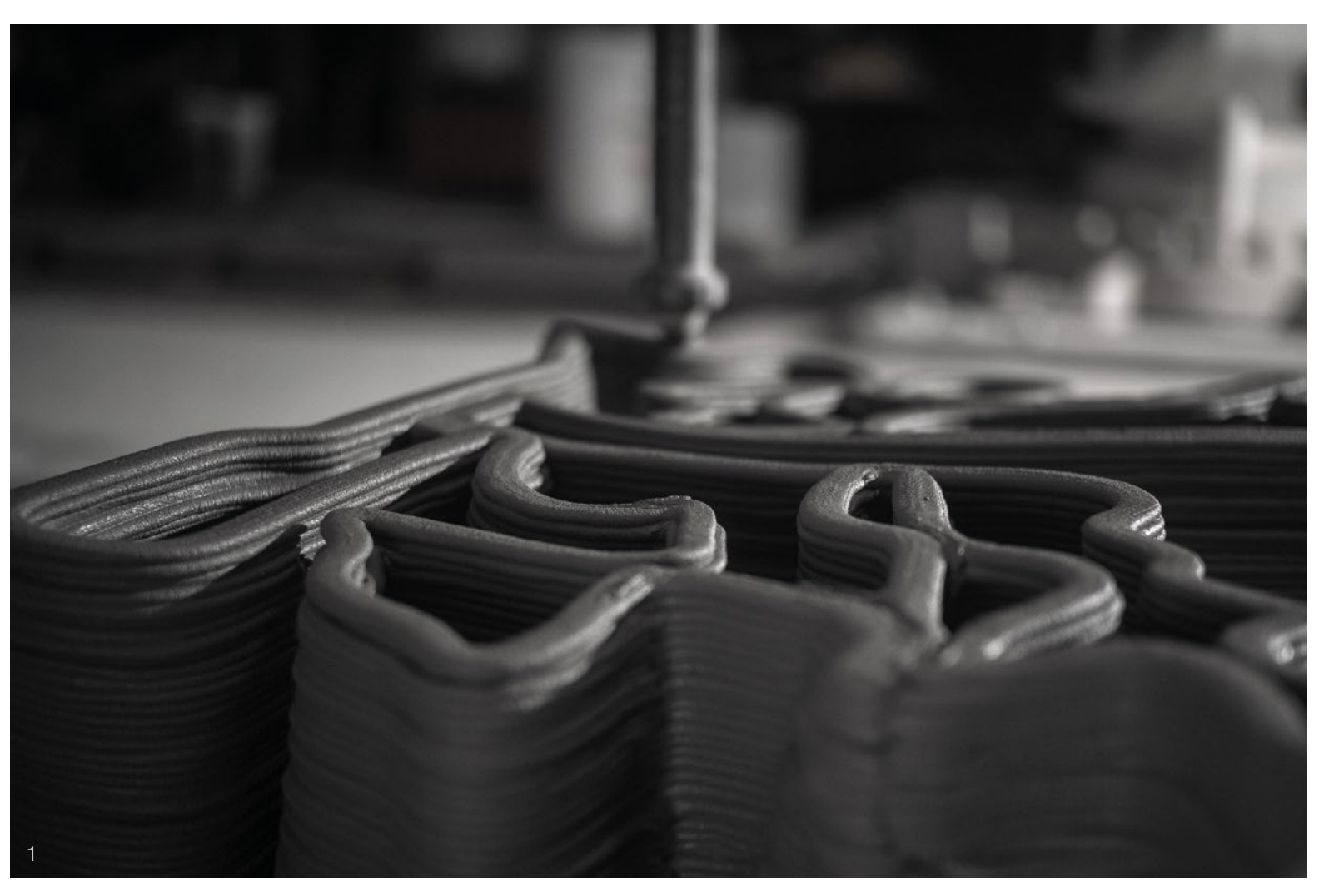

and have led to the manufacturing of several products as part of completed construction projects. Given the practical dimension of these case studies, they provide complementary information to academic research that can be used for further reflection on the industrialisation of such technologies.

Current Advancements in

Concrete 3D-Printing Processes

\section{Practical Aspects}

The first manufacturing systems developed for concrete 3D printing - by precursors such as J. Pegna (Pegn, 1997): Contour Cresting (Khoshnevis, 2004 Loughboroug (Cnversity (Buswet al, 2007); on a variety of manufacturing systems, for both the on a varlety of mand theturing printing nozzle and the motion system. Areview of devalous 3D-printing manum in Althou so far can be found in Duballet et al., 2017. Although the technologles discussed in this paper are often referred to as 'concrete 3D printing, a more accurate description is 'cementious materials $3 \mathrm{D}$ printing. The development of various cementilious material formulations has accompanied the development of the first 3D-printing manufacturing systems, with each research group developing a specific formulation tallored to its needs and system characteristics. In recentyears, The first manufacturing systens developed for as cement manufacturers have become interested in the topic, several ready-to-use 3D concrete printing (3DCP) mixes have been developed and are now marketed. Various 3DCP mixes are available, but the materials used of existing mixes and rheological properties With the development of $3 \mathrm{DCP}$ manufacturing systems and adequate mixes, numerous applications have also been studied and developed 3D printing has been experimented with at the scal of larse buildings and architectonic elements such as columns, stairs and floors (Gaudilière et al, 2018 and Rippmann et 2017). Products dereter for public both on product design and new structurat possibed A detaled revew of possible bulding syst possibilies. A detained retew of poss be buld ing systems and 2017.

\section{Challenges}

Prototypes and projects built over the past few years have demonstrated both the viability and potential of 3DCP technologies. Several of these examples have been built by 3D-printing companies in partnership with both cement manufacturers and construction companies, demonstrating the all have the same general rheological properties. A review for public works indoor and urban fur inture, and build 

research on 3DCP processes and the conditions of production of projects points to challenges that mus be tackled for industrial-grade 3DCP to fully mature and develop.

Beyond existing technical challenges, a review of which can be found in Buswell et al., 2018, the issues still to be confronted are, in the main, linked to longestablished habits in the construction industry and the inertia that can be encountered in large industries. Despite the pervasion of new technologies, most of these digital tools have been adopted for the design stage and management of building sites. The 'digital turn' is based on the notion of 'non-standard production' (Cache, 1998), whereas the construction industry relies on 'standard production'. A major paradigmatic shift must, therefore, happen for robotic construction processes to be fully adopted. While this change is under way - as the construction industry is, in part, divided up into many small business structures it has not yet been fully assimilated. Furthermore, this evolution must be accompanied by the creation of new (currently non-existent) regulations, particularly for 3DCP. Such regulations will facilitate the adoption of digital technologies by all stakeholders in the industry, including insurance providers.

Where existing research has demonstrated the usefulness of 3DCP in areas such as material savings (Rippmann et al., 2017 and Duballet et al., 2018) and its potential to reduce the environmental cost of building methods, the assessment of the impact of robotic technologies remains a major challenge. Confrontin these challenges will enable $3 \mathrm{DCP}$ potentials to blossom. Industrial developments by prominent companies hint the potential for greater productivity in the construction industry, which has been a notoriously problematic issue in recent decades. Finally, beyond the possibility of dealing with current construction and environmental challenges, experimenting with the use of $3 \mathrm{D}$ printing in architectural design could lead to the development of new formal and structural languages in the discipline.

\section{XtreeE Case Studies}

The XtreeE Printing System

The printing system developed by XtreeE, with which the case studies presented in this chapter were produced, is composed of four distinct parts: a computer supervising the 3D model and toolpath; a mixing unit for the 3DCP mix; a monitoring system for the dosage of the mix and additives, and the printing head developed by XtreeE, mounted on a six-axis robotic arm. Further details on the process and system can be found in Gosselin et al., 2016.

\section{Maritime Engineering: Artificial Reef}

The first case study focuses on design and fabrication methods employed by a multidisciplinary team, involving architects, additive manufacturing specialists, generative designers and marine engineers to produce a $1 \mathrm{~m}^{3}$ artificial reef, shown in fig.2. In February 2018, the reef was immersed in the Calanques National Park in France, as part of the REXCOR research project. The aim of REXCOR is the restoration of the rocky shoals of the Cortiou cove, into which Marseille city sewage has been discharged since 1896, impacting on the marine environment

With the aim of restoring a lost ecological habitat, this biomimetic porous reef mimics one of the richest environments in the Mediterranean Sea,

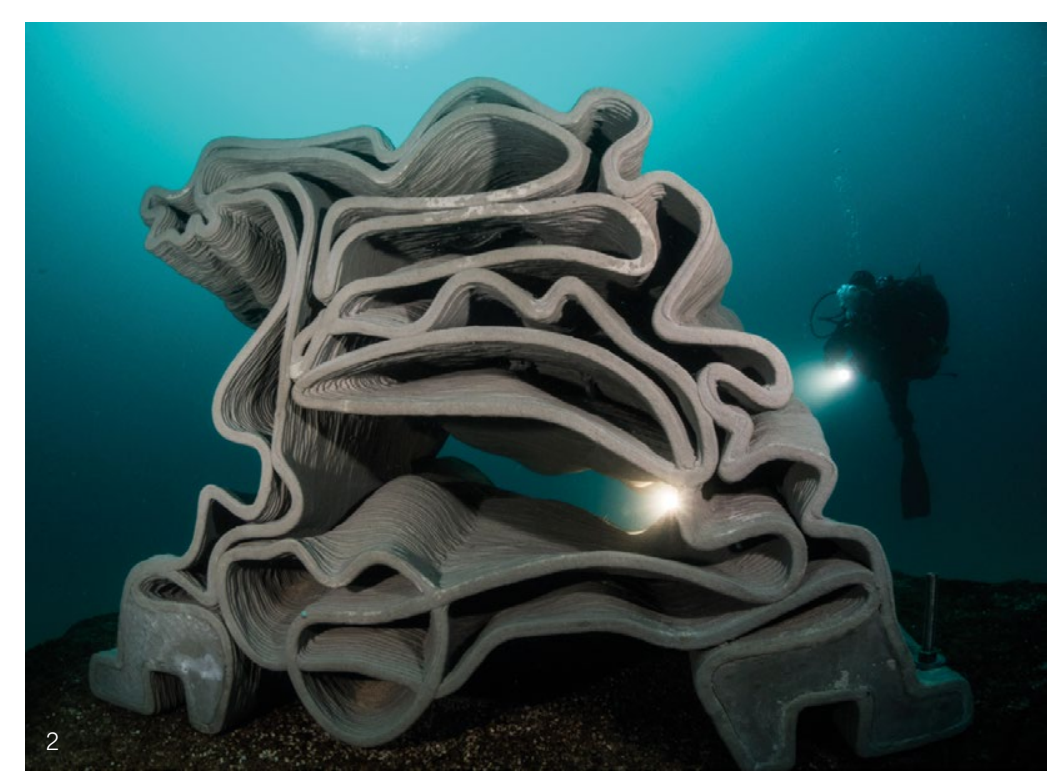

2. The artificial reef just after immersion. 3. Stormwater collector installation.

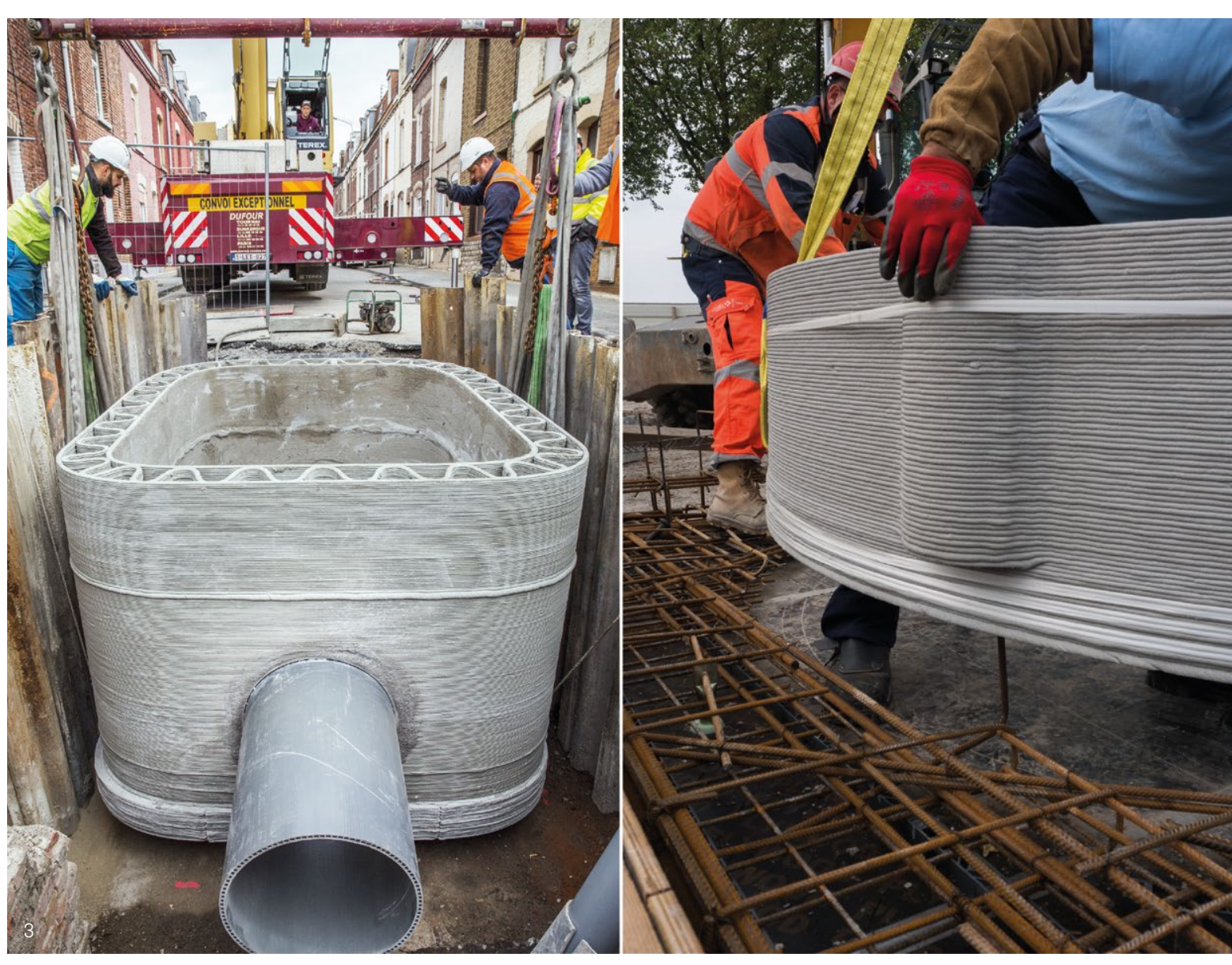

the Coralligenous habitat. This marine habitatmade of a structurally complex and dense biogenic substrate - took hundreds of years to form and is known to be a shelter for thousands of species, including fish, crustacea, coral algae molluscs, etc. Artificial concrete reefs are traditionally produced by assembling concrete slabs, drastically reducing the complexity of the avcre shabs, drand with it marine life devexment $3 \mathrm{D}$ printing allows for freater possibilities, enabling marine ensineers allows forgear wo maine to design complex talor-made multiplesca cavitien, un processes but critical to
life in the targeted area.

A specific design method for the reef was developed, with the printing system's constraints - including with the printing system's constraints - includin a maximal slope angle and hecontinuous How of concrete - and he biomimetic constraints originating from the targeted ecological habitat in mind. The prototype was printed sideways and consisted of a continuous, intersecting sheet of concrete, which considered the constraints while maximising the diversity of sizes and shapes for the cavities. On this occasion, an innovative work klow had been implemente with their differing areas of expertise needing to be put together to create the final geometry of the reef.

Although the immersion of the artificial reef presented here is recent, with regard to marine colonisation, traces of adoption by several species from the area can already be observed, confirming the otential of 3DCP technologies for future ecological engineering and restoration projects.

Public Works: Stormwater Collectors The second case study is a series of $3 \mathrm{D}$-printed Thermwater collectors for the cities of Lille and Roubaix in France in 2017 and 2018 (fig.3). These collectors are part installed onsite, addressing specific public works' needs. The idea for the collectors originated in a workshop that brought together public-works company Point.P TP and XtreeE to study the potentials of 3DCP. This series of prototypes and their use in real-life situations has underlined the potential of 3DCP in public works.

The first collector prototype was 3D printed and installed in partnership with Point.P TP, followed by three more, built according to the same manufacturing of a larger series of prototypes produced by XtreeE and 

road. The recourse to 3D printing enabled a drastic reduction in onsite intervention - two days, instead of the usual two weeks - thus reducing inconvenience and traftic disruption. The initial design was improved upon the following three collectors, with the development of a lifting system that consumed less material. The device developed for this was also used to protect the existing subterranean grid made of ancient brick vaults. As these were susceptible to damage by a standard intervention, the use of $3 \mathrm{D}$ printing enabled a connection detail to be manufactured that preserved the vaults, despite their structural weakness.

These prototypes provided an opportunity to develop a work Hiow integrating several design teams and building sites. The collectors themselves represent an interesting experiment that integrates $3 \mathrm{D}$ concrete printing into standard industrial workflows and methods; for the fabrication of the collectors, the team applied a mixed manufacturing technique based on a cast and printed reinforced concrete slab. Further description of the project and the mixed manufacturing technique can be found in Gaudillière et al., 2019a.

Architectonic Elements: Freeform Truss Pillar The production of architectonic elements raises many issues regarding $3 \mathrm{DCP}$ processes. Several research teams and companies are developing technologies with the goal of increasing the scale of the printing area, in order to produce increasingly larger buildings in a single print. Others favour prefabrication, often onsite as is the case in the pillar presented here. Although advocates of the use of $3 \mathrm{D}$ printing in construction often highlight the idea that it could lead to the disappearance of connections for building parts, this may be a of 3DCP and other manufacturing methods appears to be industry, whereas advocating for continuous, very large-scale 3D printing might prevent this. Developin smaller-scale architectonic elements, therefore is perhaps the optimal way to mix methods, both for the fabriction of the The pillor (fig A) is a $4 \mathrm{~m}$-hish collumn madeThe pillar (fic) is a am-high colum made of an in foncrete. The decision to castconcre was made constuction at the time. This resulted, as shown construction at the time. in Table 1 , in an augmentation or the amount of matter used in the pillar. A detalled description of the manufacturing method is avallable in Gaudilitiere et al., 2018, and in Buswell et al., 2018. The work How was similar to those in building projects and was, therefore, much more linear. The decision to resort to $3 \mathrm{D}$ printing was made much later than in the two other case studies presented here.

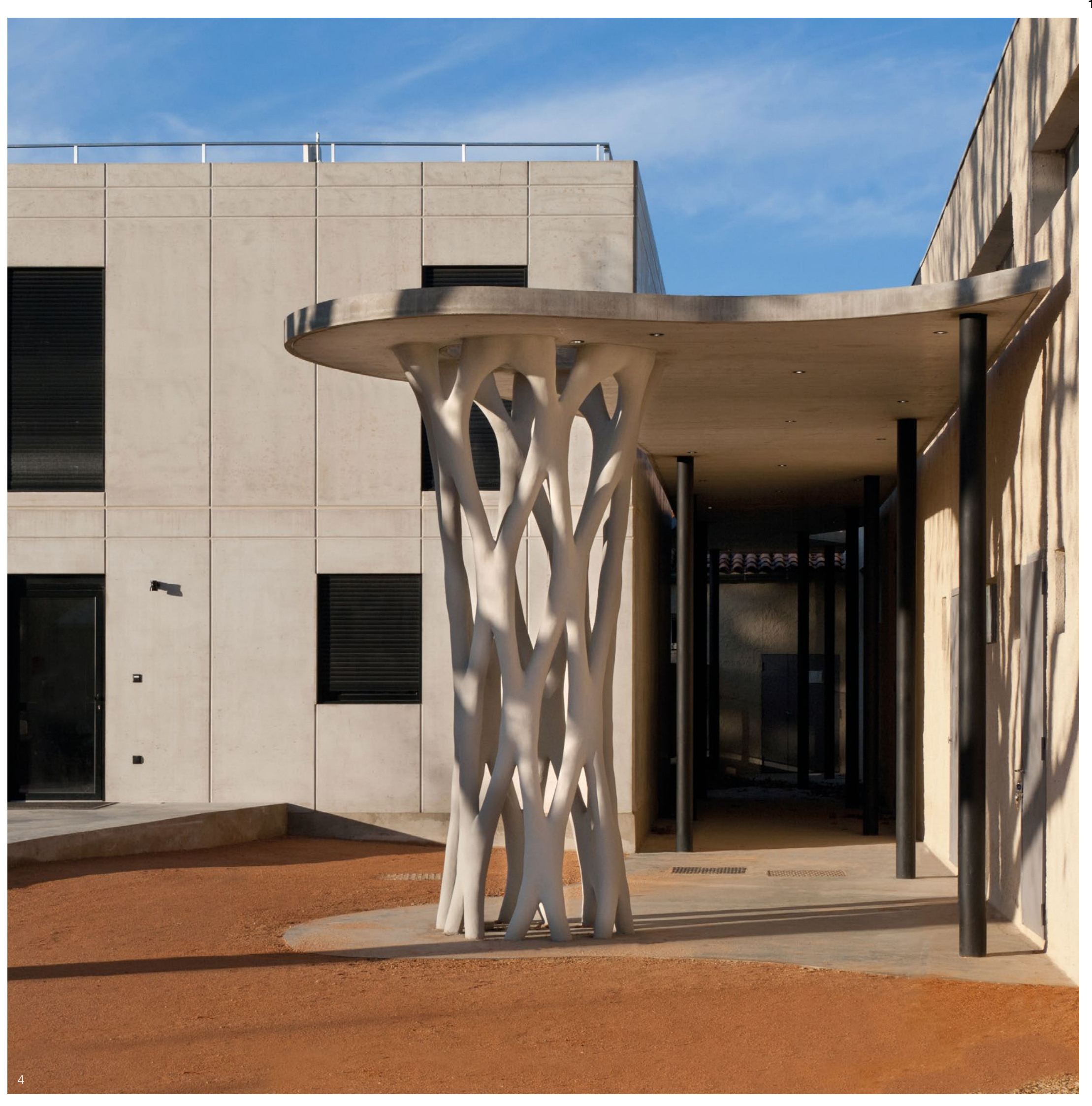

163 


\section{Project}

Artificial Reef

\section{Production Time (\%)}

\section{Material Consumption (\%)}

Collectors

Pillar

Urban Furniture

Average

Table 1. Production time and matter

consumption for projects in compariso
to traditional production methods.

\section{Production Times and}

\section{Material Consumption}

A study has been conducted to compare production time and materiat consumption for 3D-printed objects and for traditionally-bult equivalents. Objects compared include the three case studies presented in this chapter, as well as prototypes for urban furniture. Details can be found in Gaudillière et al. 2019b, while results are given in Table 1.

\section{Conclusion}

This paper presents three projects developed by the company XtreeE, in different contexts, as well as information on the different participants involved, manufacturing techniques employed, production time and material consumption. Through the description of these three case sudies, this paperattempts to re the experience in the field to existing research and challenges identified in the development of $3 \mathrm{DCP}$, for a better understanding of possible industrialisation routes. Case studies, such as the ones presented in this paper, highlight several important factors for the development of environmental design guidelines. Case studies stress the need for regulation and for a fine-tuned control of optimisation processes, accompanied by said design guidelines. The workflows presented here advocate for he combination of 3 DCP to already pre-existing techniques and for close collaboration between traditional stakeholders and new players, prompted by the development of 3D printing.

By combining the expertise of stakeholders in the industry and the mastery of robotic technologies developed by researchers, such as 3DCP, design guidelines could be established that minimise the of productivity and efficiency could be reached, alongside significant improvements in the quality of the built environment. negative impact of construction activities. New levels
The XtreeE team would like to thank the partners that have helped make the projects presented in this paper posst Stormwater collectors: PointP TP. La Sade Artificial reef: Seaboost. Krypton pillar: EZCT Architecture \& Desig D concept.

Bibliography

Buswell, R.A., Soar, R., Gibb, A. and Thorpe, A., 2007, "Freeform Construction: Megacale Rapid Manulacturing for

Construction, Vol. 16, p.224-231.

Buswell, R.A., Leal de Silva, W.Z., Jones, S.Z. Concrete Extrusion: A Roadmap for Research' in Cement and Concrer
Research, Vol. 107 (May 2018).

Cache, B., 1998, 'Objectile: Poursuite de Philosophie par d'autres Moyens?' in Rue Descartes, No. 20, p.149-15\%.

Carno, M. (ed.). 2012. The Digital Turn in Architecture 1992-2012: AD Reader,

Cesarettig $\mathrm{G}$. and Pambaguian, L., 2014, 'Building Components for an Outpost on the Lunar Soil by Means of a Novel 3D Printing
Technology' in Acta Astronaut Vol p. $430-450$.

Duballet, R., Baverel, O. and Dirrenberger, Construction, Vol. 83, p.247-258.

Duballet, R., Baverel, $\mathrm{O}$. and Dirrenberger, $J$ Robotic Mortar Extrusion' in Structures, Vol. 8, p.41-47.

Gaudillière, N., Duballet, R., Bouyssou, C., Mallet, A., Roux, P., Zakeri, M. and Dirrenberger, J., 2018, 'Large-Scale Additive Manufacturing of Ultra-High-Performan Truss-Shaped Pillars' in Willmann P., Hutter, M., Byrne, K. and Schork, T. (eds.) Robotic Fabrication in Architecture, Art and D.459-472,

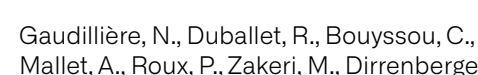
J., 2019a, 'Building Applic Forimworms Obtaned through Large-Scale (a) Nazari, A. and Nematollahi, B (eds.). 30 Concrete Printing Technology, Oxford, Concrworth-

Gaudillière, N Duballet, R, Bouyssou, C. Mallet, A., Roux, Ph., Zakeri, M. and Dirrenberger, ,., $2019 \mathrm{~b}$, 'Bénéfices temps d'impression $3 \mathrm{D}$ béton pour des applications dans le domaine de la construction' (conference paper from Construction. Quelleation Adualitive pour la 7-18 January 2019, Champs-sur-Marne, France).

Gosselin, C. Duballet R. Roux P. Gaudillière, N., Dirrenberger, J. and Morel, $P$. 2016, "Large-Scale 3D Printing of UltraHigh-Performance Concrete: A New Builders' in Materials and Design, Vol. 100, p.102-109.

Gramazio, F., Kohler, M. and Willman, J, Change Architecture, Zurich, Switzerland, Park Books.

Khoshnevis, B., 2004, 'Automated Construction by Contour Crafting-Related Aubotics and Information Technologies' in Pegna, J., 1997, Exploratory Investigation of Solid Freeform Construction' in Automation Rippmann, M., Liew,A. and Block, P., 2017, 'Structural 3D Printed Floor' in Yuan, P.F., Menges, A. and Leach, N. (eds.), Digital

Roussel, N., 2018, 'Rheological Requirements for Printable Concretes' in Cement and
Concrete Research, Vol. 107, p.76-85. 
Integration of Material and Fabrication Affordances within the Design Workflow

Giulio Brugnaro, Sean Hanna, Peter Scully and Bob Sheil

The Bartlett School of Architecture, UCL

Silvan Oesterle

Rippmann Oesterle Knauss GmbH (ROK)

\section{Introduction}

In current design practices, the lack of feedback between the different steps of linear design processes forces designers to engage with only a limited range of standard materials and manufacturing techniques, leading to wasteful and inefficient solutions. Focusing on robotic carving with timber, this paper investigates the extent to which the early-stage integration of material knowledge as part of design-to-manufacture workflows makes it possible to explore novel, previously unavailable, design solutions informed by the fabrication process.

\section{Background}

The integration of digital fabrication technologies within design practices is challenging the separation between designing and making in current production workflows (Koralevic, 2008: Carpo, 2011). A novel sensibility tows materia and tool technologies has become centar part of the architectural discours (Detanda 2002; Menses, 2015), where designers (De asked to envision perfer, 20 in performance-driven processes bridging the digital and physical realms, rather than focusing static forms (Gramazio and Kohler, 2008). Simulation tools and robotic fabrication technologies are regarded as enabling frameworks to establish feedback loops driving the design and making of artefacts (Maxwell and Pigram, 2012).

The development of sensor devices to record and reconstruct manufacturing tasks has made it possible to adopt machine learning models, such as Artificial Neural Networks (ANNs), that are able to synthesise and integrate knowledge to support decision-making, based on the live material and process data (Lu, 1990;

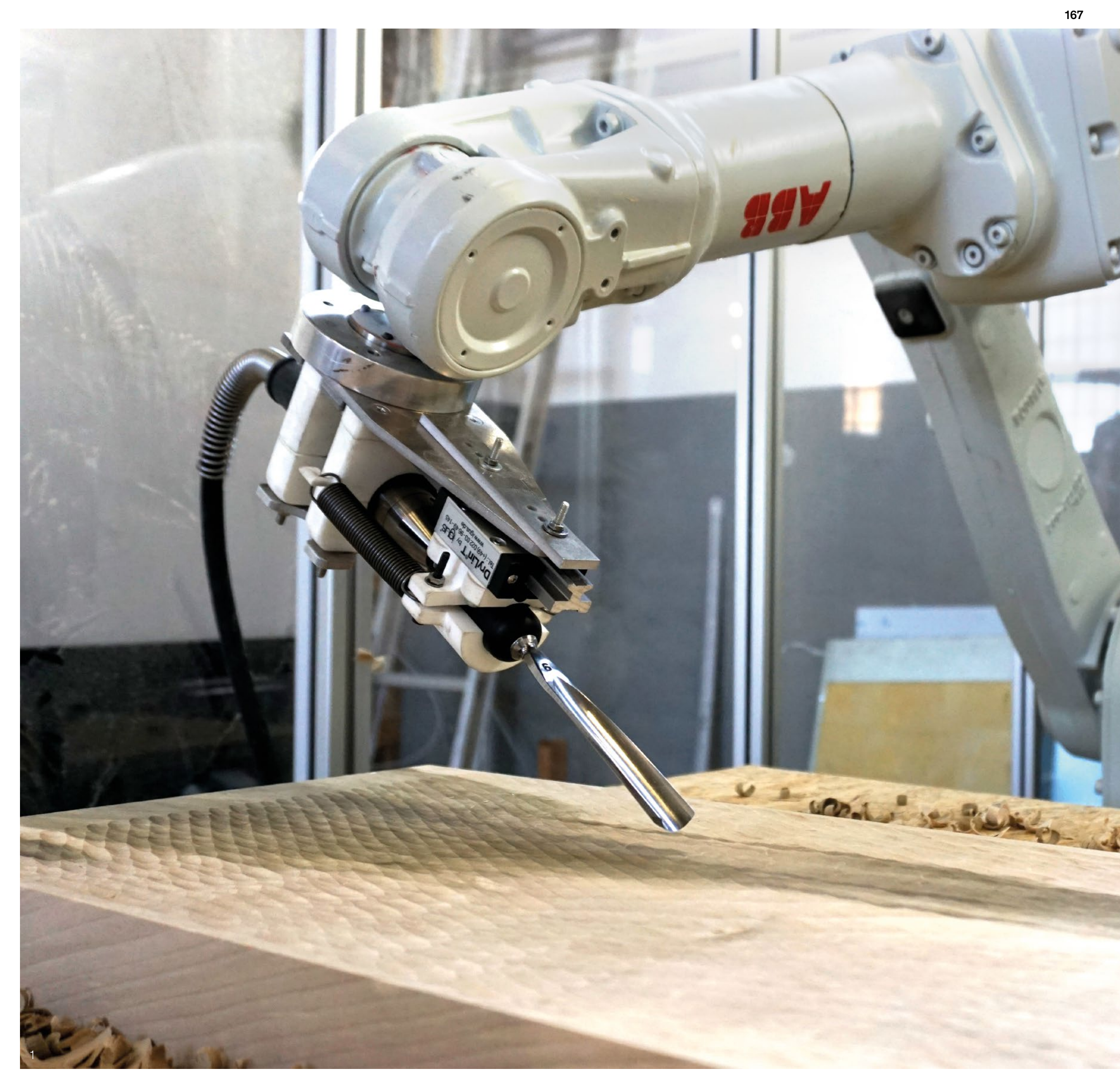



(n) parameters to be optimised in relation to the physical output of the task, increasing the overall efficiency of the production process (Tsai et al., 1999). The encapsulation of such knowledge into a transferable package offers the opportunity to integrate with a design interface, allowing for exploration of design opportunitie (Hanna, 2007; Tamke et al, 2018).

\section{Methods}

This research used real-world fabrication data, collected by human experts and autonomous robotic sessions, to derive a more accurate geometrical prediction of carvin operations on timber. To achieve this, the methods developed present a series of training procedures for a robotic fabrication system, where the instrumental and material knowledge of skilled human craftspeople is captured, transferred, robotically augmented and finally integrated into an interface that makes this knowledge available to designers.

The training process is based on a cycle of three main stages:

Recording: The acquisition of fabrication data is structured around a series of carving sessions to collect into a dataset the combination of fabrication parameters driving the carving operation (i.e. tool/ surface angle, tool/grain direction angle, force feedback, input cut length, input cut depth) and pair them with their respective outcomes measured as 'actual length', 'width', 'depth of the cut' and 'total removal volume'. Such information is captured, in real-time and retrospectively using an array of motion capture (MOCAP) cameras to track the position and orientation of the carving tools and 3D-photogrammetric techniques to reconstruct in a hishly detaled mesh geometry the result of the carving operations.

2. Learning: The collected datasets are used to train supervised machicted datase ts a used to train a supervised machine learning model, or ANN, whose main learning objectivis to predict the geometric defined toolpeth and the series of fablic anserperamed tool path and he series of fabrication parameters descibed above, as well as generating a

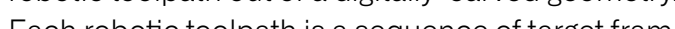
Each robotic toolpath is a sequence of target fram which defice the position and orientation of the carving gouge along the cut. Given a sequence of target frames, the trained ANN predicts, at each frame, the geometric oulput parameters of the cut (length, width, depth), considering the influence of material properties determined by the wood

the resulting angle of the carving operation in relation to the grain direction.

3. Fabrication: The trained ANN represents a packag of instrumental knowledge that can be transferred, reused, extended and, most importantly, integrated within an interface to digitally evaluate multiple design solutions informed by tools and material properties before moving to the production stage.

The training workflow should not be considered a linear progression from the recording to the fabrication stage but rather as a knowledge platform that can be remodelled over several cycles with new fabrication data, trained to improve its prediction performance and applied to various design tasks.

\section{Industry Experiments}

Collaborations with two industry partners - ROK Architects and BIG - provided the opportunity to apply these methods into established workflows and develop a catalogue of design explorations for a wide range of applications, from furniture to building components of larger assemblies.

The secondment at BIG took place concurrently with the installation of two industrial robotic arms in the office space. This enabled the team of designers to directly engage with the training workflow for robotic carving operations through an extensive series of experiments exploring the potential of integrating fabrication and material aspects at an early stage of the design process. During the training, designers started by selecting. three substantially different wood species (Lime, Tulip and Oak) for both their aesthetic qualities and mechanical properties. The second focus of the investigation was on the interaction of a set of differen carving tools with the material properties, such as srain density and directionality of the selected wood species. Each wood species dataset counted between 430 and 460 robotic carving operations. Each training board (300 mm $\times 400 \mathrm{~mm}$ ) counted between 32 and $36 \mathrm{cuts}$ (300 m $\times 400$ mm) counted between 32 and 36 cuts 列 most time-consuming part.

sor data collected during the recording sessions, was integrated into a

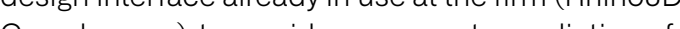
Grashopper), to provide an accurate prediction of the carving operations and explore multiple material solutions in terms of patterns and moving to the production stage.

For each fabricaled panel, several digital designs were explored through the ANN-based simulation of the outcome geometry. The tree-like structure of the investigations allowed the design to advance through
2. An example of the 'what-if' scenarios analysed during the design of carved pan introduced by fabrication and material
Houten, 2006) presenting, at each stage, a comparison of the effect generated by a specific fabrication or material parameter on the overall design as inherited from the previous stage (fig.2). Such explorations ent the analysis of geometric pattern variations, wood and specific fabrication parameters (e.g. tool/surface angle) which would significantly affect the resulting length, depth and width of the cuts composing the overall pattern.

Once robotically fabricated, the selected carved geometries were compared using a deviation analysis with the respective simulation to assess the predictive abilities of the system (fig.4).

The secondment at ROK set out to further test the machine learning based design tool the associated workflow and the robotic-carving process (fig.5) through the design of a piece of furniture for a gallery space, where a composition of carved boards created a series f platforms to display small items (fig.6).

The demonstrator was used to address challenges such as the balance between top-down decisions and features emerging bottom-up from properties of the material, using the simulation framework to visualise unexpected results and to adjust the fabrication parameters to match the prescribed design intentions and requirements. The ANN networks necessary for the simulation were trained using Lime - a light-coloured For each of these fabrication steps, the trained ANN 


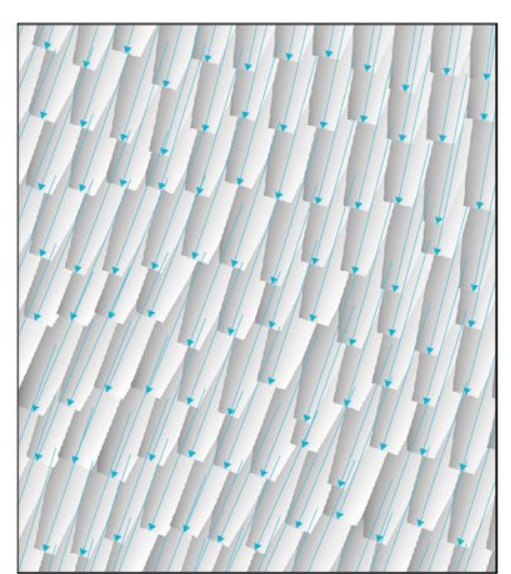

\section{Wood Species: Lime}

Wood Species:
Grain: $30^{\circ}$
Tool: $G 920$

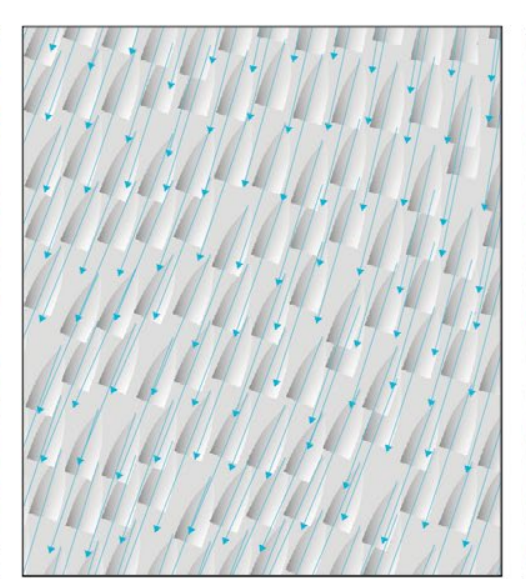

Wood Species: Oak
Grain: $30^{\circ}$
Tool: 6920

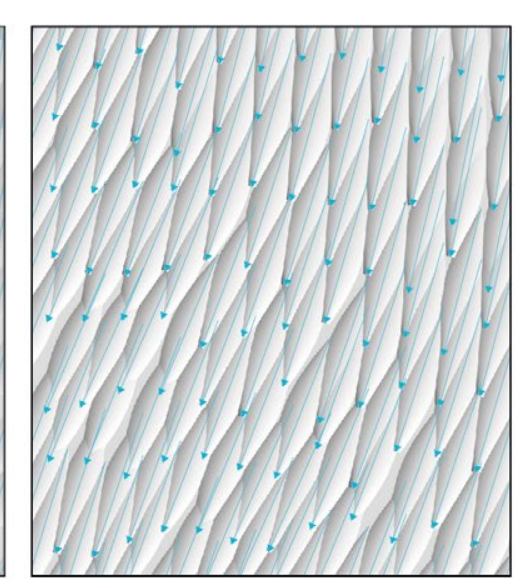

Wood Species: Tulip
Grain: $30^{\circ}$

Grain: $30^{\circ}$
Tool: 6920

3

generated a simulation of the carving outcome that evaluated, at each step, the impact of the design choices before production. Each carved board, while following a similar design logic, presented local individual features and variations in the pattern arrangements of parallel flutes due to the wood grain behaviour on the control of input design parameters. As in the previous case study, each carved board was reconstructed digitally postfabrication using photogrammetry and compared to the ANN-based prediction, generating a gradient-based mapping of the deviation between the two This made it possible to validate the tool and assess the impact of its application as core elements of the digital design process (fig.7).

Results

The catalogue of material evidence and digital experiments, performed during the secondments, suggests that the curation of the training process suggests that the curation of the training process
represents the keystone of the entire design workflow. Following the selection of wood species, the search domain defined, at an early stage, relevant material domain defined, at an earlystage, relevant material that dies, caning tools and rabication parameters that direolly deting The later digital design stage.

enarios, driven by tools and materal propertes, would not be possible using purely digital geometric considerations, rather than a collection of real-world fabrication data. The evaluation of the impact of choices, such as the selection of a specific wood species, therefore, allows a series of unlocked and supports a better-informed decisionmaking process

While the prediction of single carving operations is accurate to within a fraction of millimetres, the analysis of complex fabricated patterns shows a higher deviation between the ANN-based prediction and the

photogrammetric reconstruction of the carved board. The main reason for this is, likely, due to the combined effect of overlapping cuts whereby mechanical

conditions are generated that are not present in the single-cut configuration. Nevertheless, the ANN-based simulation showed an overall high level of accuracy in its predictions, proving its ability to correctly model the its predictions, pr different materials and fabrication model the impact of different materials and fabrication afford

on the geometric outcome of carving operations. scenario does not represent, necessarily, the end of the design process but rather, becomes the starting point design process but, rather, becomes the starting point upon the initial fabricated evidence. Following tre-like upon the inilal fabricated evidionce. Following a tree-l structure, the design-to-fabrication process is rarely revised especill if new man erly stage can always be The sim The sia material-aware solutions ahead of the fabrication stage; however, this needs to be carefully balanced with the more conventional top-down, geometry-driven design approach. The tools developed here allow the original design intention to be achieved through the optimisation of individual fabrication parameters or, alternatively, a more open-ended trajectory where material and a
3. Comparison of the influence of three
different wood species (Lime, Oak, Tulip) on the same digital input geometry.

4. Deviation analysis between a portion ANN-based simulation.

5. The robotically-carved boards follow parameters and material behaviours. local variations given both by inputdesite

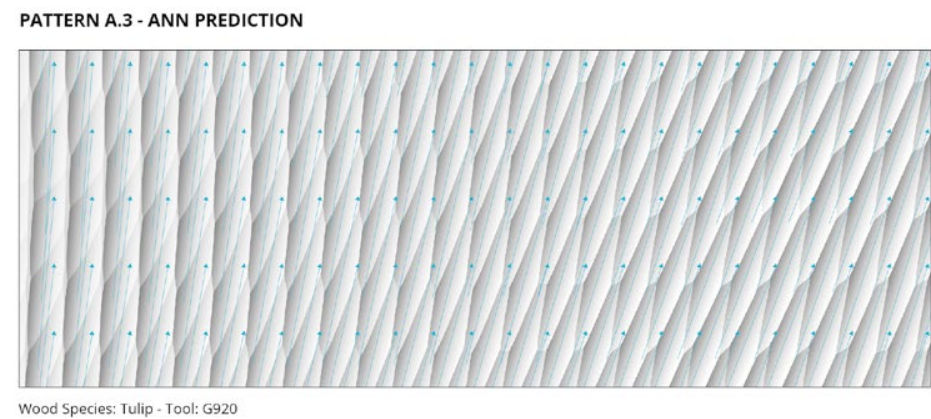

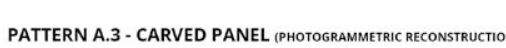
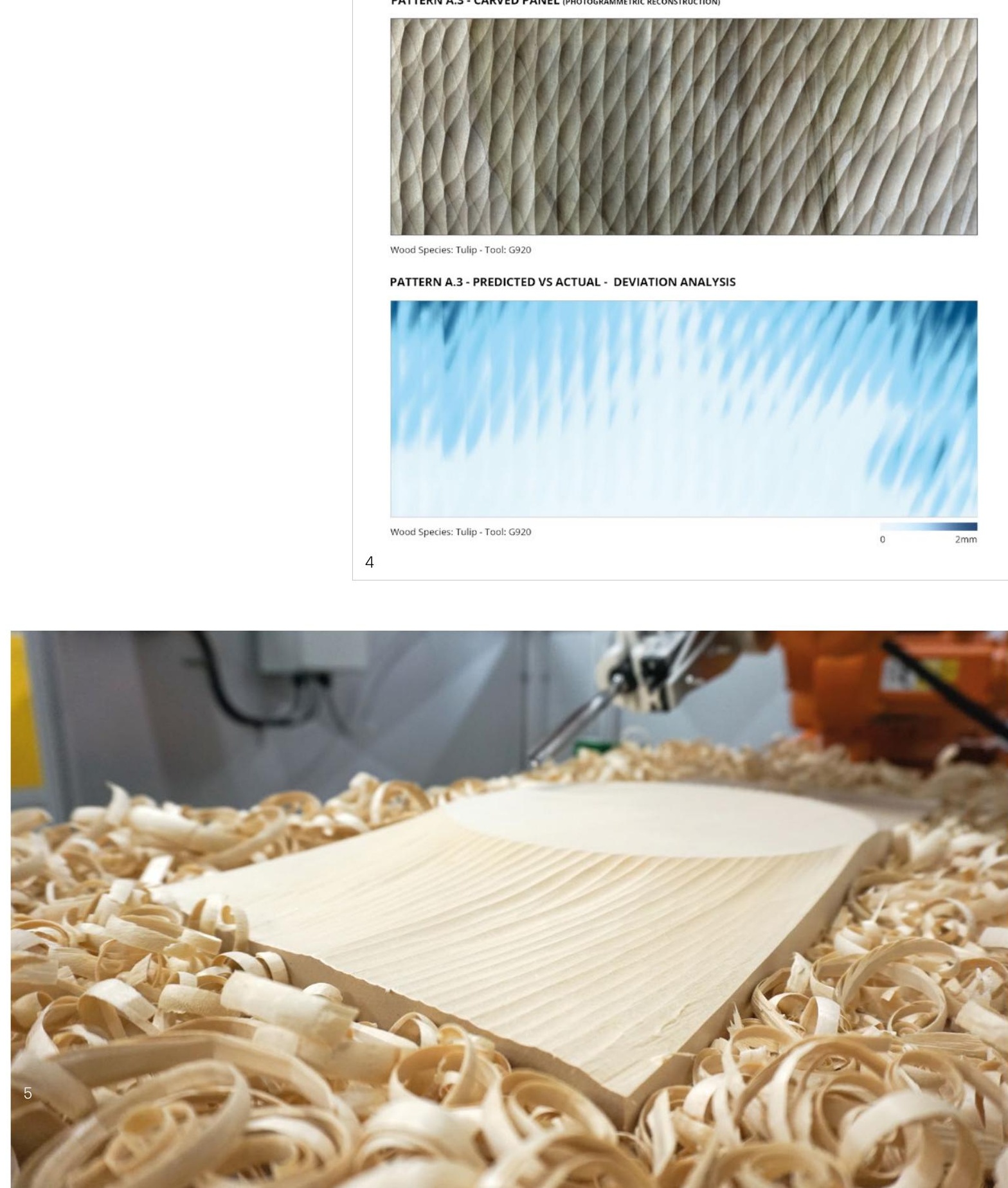


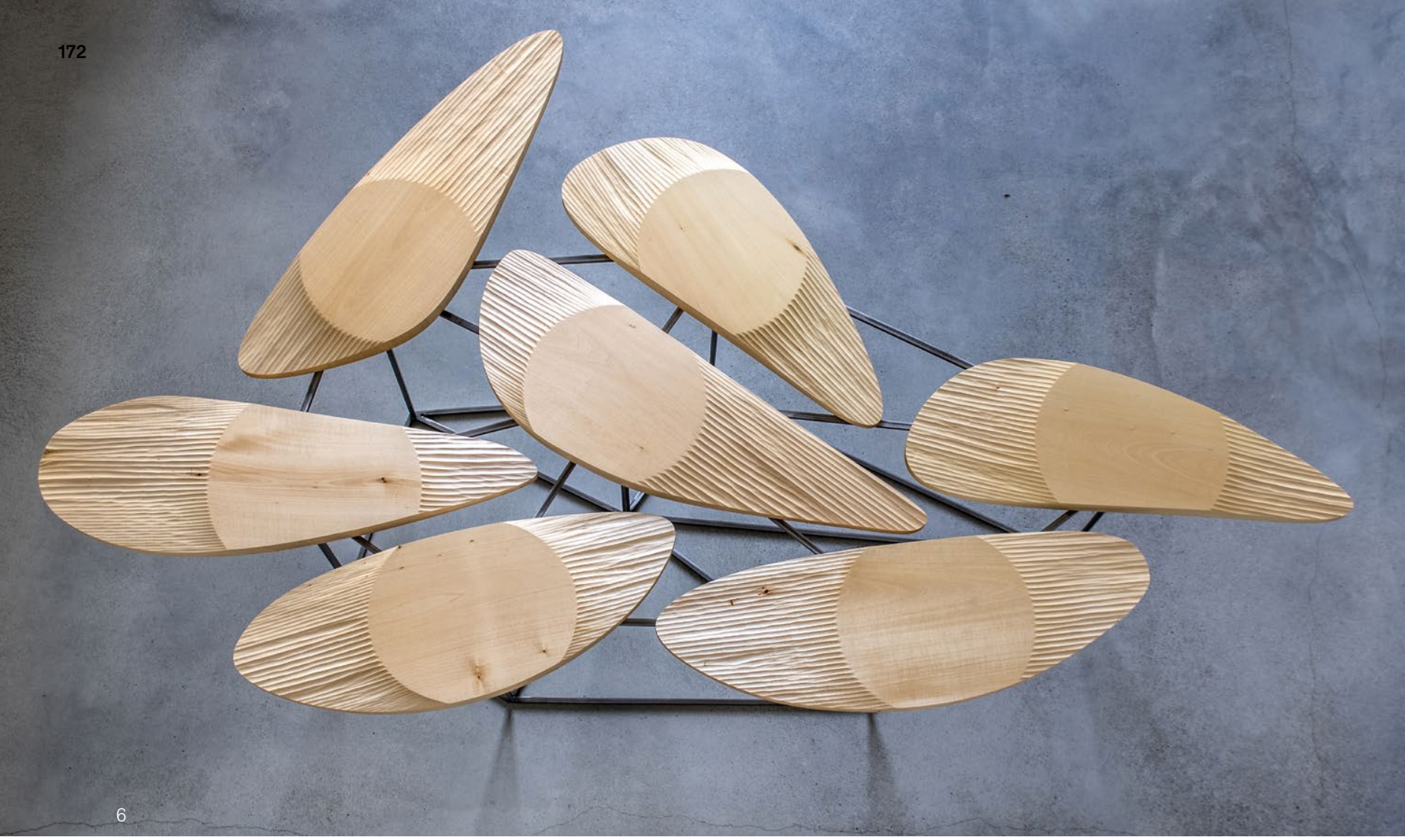

\section{Conclusions}

The main contribution of this research is to the field of applied machine learning strategies, bridging roboticmanufacturing environments and digital-design

interfaces. Although the research strategically focused on one very specific application - robotic carving with timber - the methods developed have the potentia to be applied to a wider variety of non-trivial robotic manufacturing tasks that require dexterity and a high-level understanding of the process.

Previous research has explored machine learning models being applied to optimise robotic tasks within an industrial context. The novelty of this research lies in applying similar established methods within the workflow of creative practices to augment and support the

\section{abilities of designers.}

The successful development of a series of methods to collect, process and encapsulate manufacturing knowledge and its application within a design environment demonstrates the benefits of interacting with fabrication tools and material affordances early on, to make better-informed design decisions.

From the perspective of designers, the access to packages of instrumental knowledge enables manufacturing techniques to be extended, as the trained networks significantly increase accuracy in the prediction and simulation of non-standard processes. Designers willing to engage with the curation of the training process have the opportunity to create custom-designed manufacturing workflows, validated by feedback data and statistical models. For companies, the research demonstrates the advantages of packaring knowledge making it available to all the stakeholders involved in the design-to-manufacturing workflow, to ensure fruitful communication from the outset and he avoid costly mistakes at a later stage.

BOARD
$\begin{gathered}\text { Fabricated Board } \\ \text { Photogrammetri } \\ \text { Reconstruction }\end{gathered}$

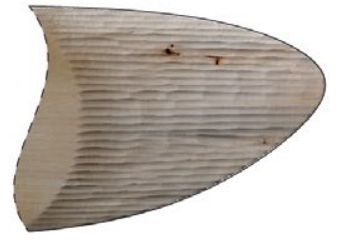

BOARDC
Deviation
Analysis

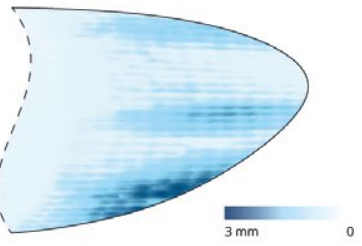

Robotic Carving
Toolpaths

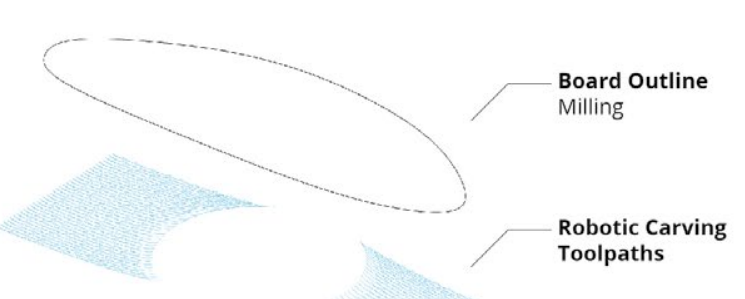

ANN-based
Simulation

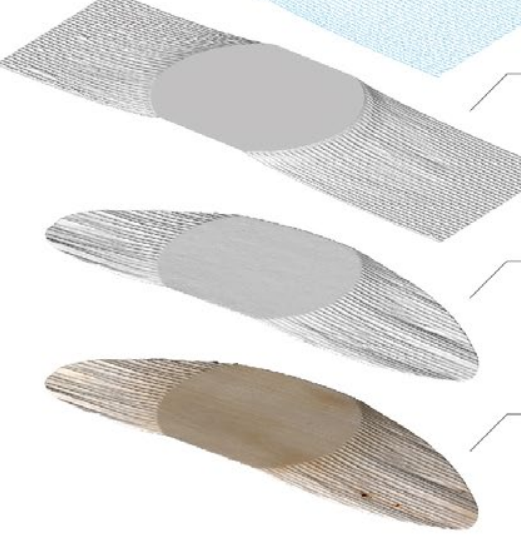

Fabricated Pane
Photogrammetrii

Fabricated Panel
Photogrammetric

Photogrammet
Reconstruction

Reconstruction
+Texture
6. The demonstrator, named Kizamu

of a series of boards used to display

small objects in a gallery.

7. The ANN-based simulation illustrates

the impact of design decisions, in relation
to the properties of the wood, and inform
the robotic fabrication accordingly
Bibliography

Carpo, M., 2011, The Alphabet and the
Algorithm, Cambridge, MA, MIT Press.

DeLanda, M., 2002, 'Philosophies of Design: J. (ed.), Verb 1 (Architecture Boogazine): Authorship and Information, Madrid,

Gramazio, F. and Kohler, M., 2008, Digital Materiality in Architecture, Baden,

Hanna, S., 2007, Inductive Machine Learning of Optimal Modular Structures: Estimating Solutions Using Support Vector Engineering Design, Analysis and Manufacturing, Vol. 21, No. 1, p.351-366.

Kolarevic, B. and Klinger, K.R., 2008, Rethinking Design and Makin in Architecture, New York, Routledge. Lu, S. C-Y., 1990, "Machine Learning Approaches to Knowledge Synthesis Engineering Automation' in Computers

van Luttervelt, C.A., Childs, T.H.C., Jawahir, I.S., Klocke, F., Future Trends in Modelling of Machining
Operations' in CIRP Annals, Vol., 47, No. 2, p.587-626.

Maxwell, I. and Pigram, D., 2012, 'In the Cause of Architecture: Traversing Design
and Making in Log, Vol. 25, p.31-40.

Menges, A. (ed.), 2015, Material Synthesis: Fusing the Physical and the Computational
(Architectural Design Series), London, Wiley.

Tamke, M., Nicholas, P. and Zwierzycki, M., Design: Practices and Infrastructure' in International Journal of Architectural

Tsai, Y.H., Chen, J.C. and Lou, S.J., 1999 'An In-process Surface Recognition System Based On Neural Networks In End Milling Cutting Operations' in International Journal
of Machine Tools and Manufacture, Vol. 39 No. 4, p.583-605.

Vaneker, T.H.J. and Van Houten, F.J.A.M Working Method in Product Design'

Working Method in Product Design' in 


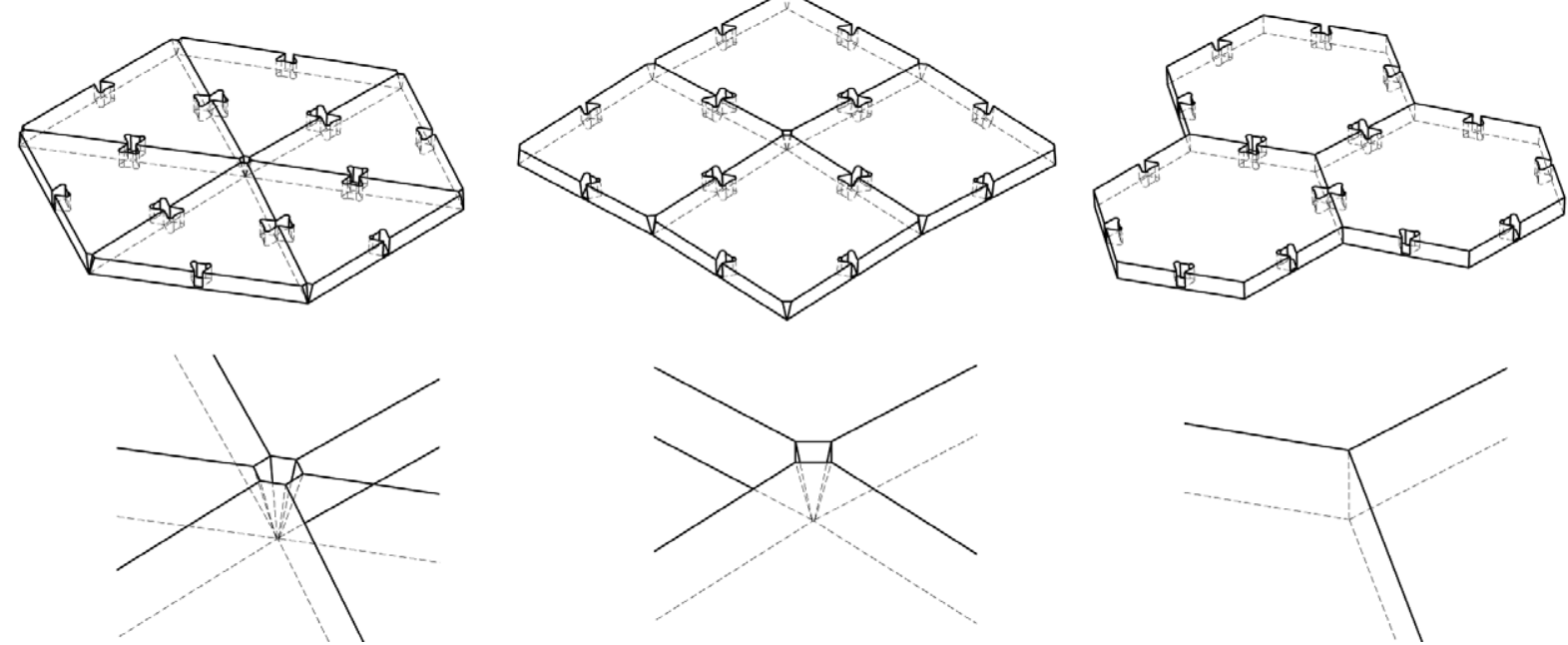

2

shows a comparison of three-, four- and six-edged plates. In this case, the number of edges meeting at the node is indirectly proportional to the number of sides of the components. From a manufacturing point of view, meshes in which a maximum of three edges meet at each node (so-called 'trivalent networks') present advantageous properties. In addition to the favourable structural properties of trivalent networks (Kübler, 2014), it is always possible to construct a mesh offset by the thickness of the CLT plate without errors (fig 2 bottom right). For other meshes, this is only possible in specia cases (parallel networks). Nevertheless, even in meshes that ( that are nathes affet by the thickness of the plate. The diagram shows how in these cases, cone-shaped cut-outs are added at the nodes On the underside, the surface always remains closed, but from a building physics point of riew, the complosely closed knoild physics poin of view the com the tivis pont mesh is ideal. In thecase of theconeshaped cut outs, he resulting cav

Apart from design considerations, another aspect in the choice of the facet-shape is its influence on the tota edge length. With a constant plate size of $1 \mathrm{~m}^{2}$, a companson of the three diferent facet shapes shows face to facet-shape. For the four edged panels, the edges are about 5\% longer, while for the three-edged panels they are about $15 \%$ longer. This determines the number of production time.
Fabrication and Assembly

The 59 components for the Klagenfurt exhibition stand were cut on a five-axis CNC system with a 9kw spindle. The convex outer shape of all of the components offers a major advantage in production, in that the entire cut of the slightly sloping edges can be made with a saw blade. This is not feasible for components with concave corners on the outer contour, which must instead be cut with the end mill, significantly increasing the time required. The cut-outs for the X-fix connectors were made with a milling cutter in five passes. To prevent the plates from moving as they normally would (vertically to the shell) during assembly of the elements, two recesses for Lamello connectors were milled, per edge, using a hand machine. For this first prototype the total production time per component was 20 minutes. With a higher spindle power, the number of in-feeds during milling could be reduced, irnificantly shortening the total production time. roduction time.

The construction confirmed the main advantage of the Timberdome system principle: within just a few important that no costly and time-consuming important that no costly and time-consuming substructure, which is namy required for freerorm panel structures, was required here. The assembly comprised two steps. First, the two prefabricated halves int the shell were assembled using 131 connectors, taking into account the size of the vehicle which would be used were assembled using 17 additional connectors. Another advantage was the simple and quick disassembly of the hours, the exhibition stand's 59 components were to transport them to the site. Then, onsite, the two parts
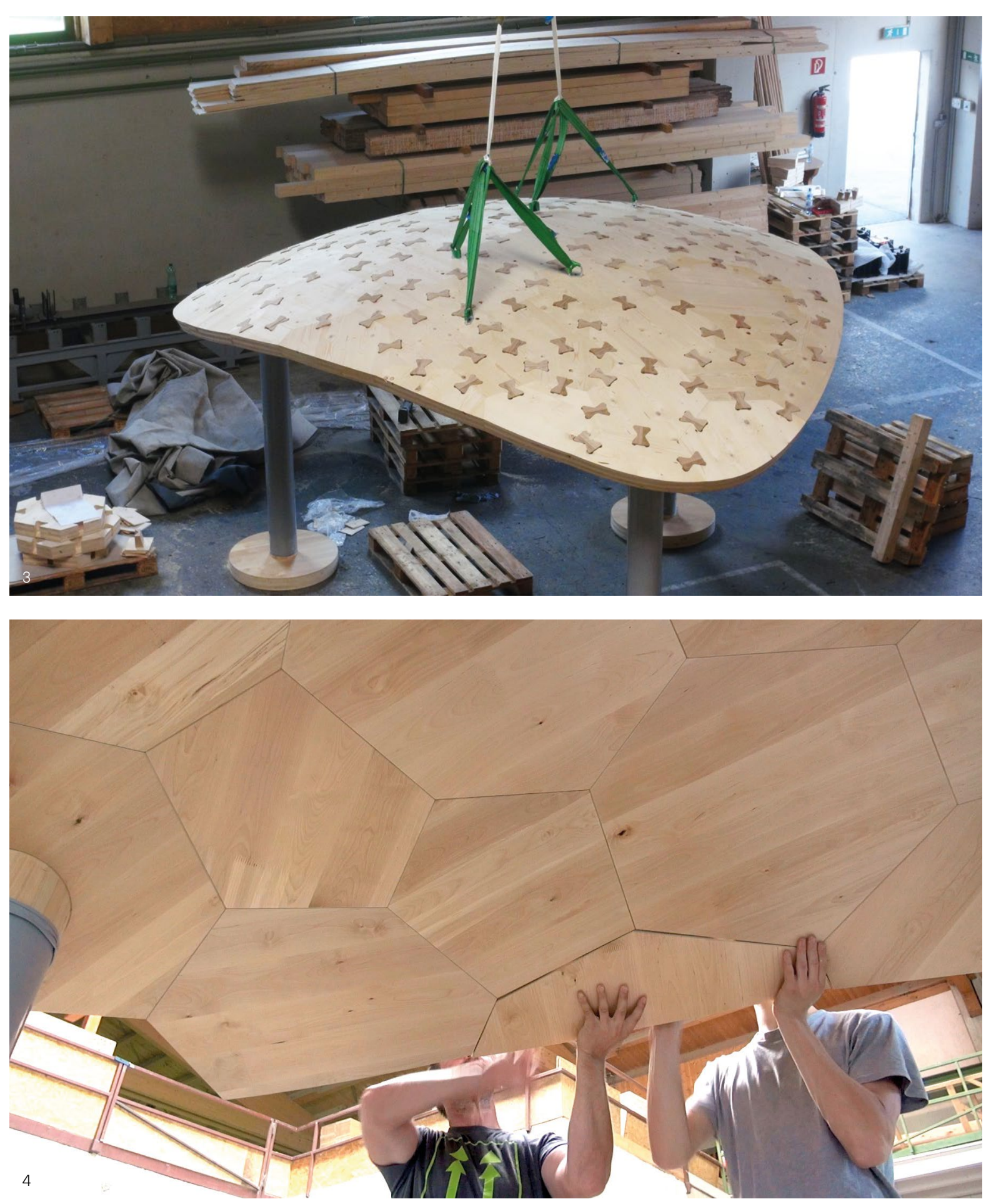

2. Comparison of three-edged, four-edged and six-edged plates.

Enlargement of the node detail.

3. 59 components fully assembled.

4. Assembly of components during test setup and interior view of 
The aim of our structural analysis was to show which spans could be realised with the X-fix Timberdome construction system, considering particularly

unfavourable loads under snowy or windy conditions. First, the load assumptions, as well as the static systems used for the calculation, were discussed. The calculation for the spherical shell were carried out on trusses as well as systems with orthotropic plates, taking into account the mechanical compliance of the connections. For our structural analysis with the Finite Elements Software RFEM, we used a dome geometry with a span of $15 \mathrm{~m}$, a height of $3 \mathrm{~m}$ and a surface area of $187 \mathrm{~m}^{2}$. The riseto-span ratio was 1:5. The uniform curvature of the dome lent itself to homogenous division into regular hexagona elements. This is important, because the 384 inner edges were each joined with one X-fix $\mathrm{C}$ connector. Increasing the number of connectors per edge is only possible in relatively large steps. To avoid weak points in the overall system, we kept the variance of the edge lengths low $(\varnothing 715 \mathrm{~mm})$.

For the subdivision of the surface, first we used an algorithm that evenly filled the doubly-curved surface with circles. We chose $1,300 \mathrm{~mm}$ as the diameter of these circles in order to optimally use the working space of the CNC cutting machine. We then created a polygon mesh based on the centre points of these circles, using the Delaunay triangulation method. This resulted in a network with equilateral triangles. Next, we generated a dual network, in which the centres of the areas in the triangle mesh formed the nodes of the dual network. This created a hybrid, trivalent polyon mesh, with 141 facets and five-, six- and seven-sided shapes.

The most unfavourable load case and the resulting deformations were calculated on the basis of the aforementioned characteristic values. Due to the load applied, the shell indented on the left and bulged out on the right. The small deformations (maximum $4.7 \mathrm{~mm}$ ) suggested there was a high overall stability of the construction system. Lons-term deformations, which can arise due to the creep behaviour of wood, as well cas vielding in the supports, were not taken into account as this modet and require werther tests. Likew in this model and require further tests. Likewise, the by the local application of force in the coupling model. system, however, are primarily the tensile and bending system, however, ae primally the tensile and bending the help of the static join betweentac replacement system. Load-bearing generally ch the side faces of CLT plate elements angles metal plate connectors were not an option either.

\section{Conclusion}

The Timberdome system is a new assembly technique for segmental shells, made from CLT. The greatest advantage of the system is its very simple, fast and precise assembly. When constructing the first prototype, two larger components were prefabricated, each with an area of $8 \mathrm{~m}^{2}$, so only a few connectors needed to be joined onsite. No substructure or formwork was needed for the construction. From a design perspective, the system allows for a variety of applications. Openings for lighting and ventilation are easy to implement in the planar plates, in contrast to concrete and brick shells. The desired shell geometry can be subdivided with different facet shapes, e.g. with three-, four- or six-edged plates. Trivalent meshes offer advantages from a structural, manufacturing and building physics perspective. The total edge length is the lowest for six-sided plates, which influences the required number of connectors and the manufacturing cost. Our structural investigations show that, based on the construction system described here and using $100 \mathrm{~mm}$-thick spruce wood CLT plates, a self-supporting shell structure with a span of $15 \mathrm{~m}$ can be realised. CLT is the ideal material with which to produce the structure, which is primarily subjected to pressure. The load capacity of the pressure surfaces is high, and a positive effect on the load-carrying behaviour is that each positive effect on the parter As a result, the concentric pressures and tension take-up and deformations are reduced. The system's redundancy - such as for the failure of connectors and diverted force transfer - is a topic ripe for future and diverted force thas for even larger spans than that attain the this proct be achieved through an optimisation of the shell geometry, thicker plates and different plate materials.

5. Geometry of the FEM test dome: span area $187 \mathrm{~m}^{2}$. Number of plates: $141 ;$; $\oslash$ p plate size: $1.33 m^{2}$; inner edges/ $X$ - in.

6. Bending moments at the couplings a.
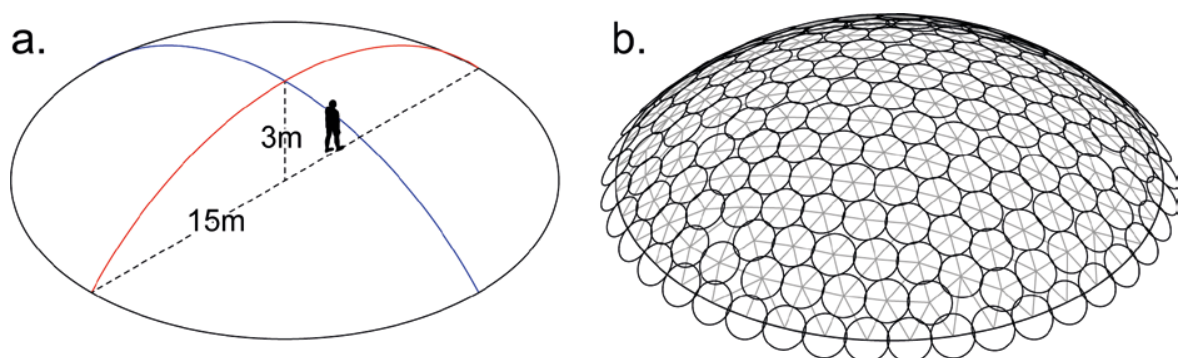

c.

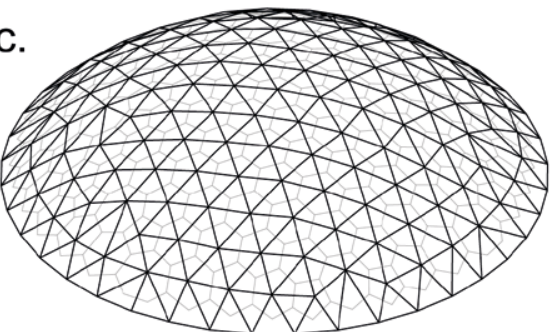

d.

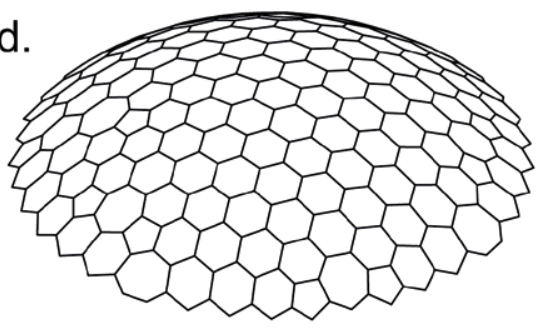

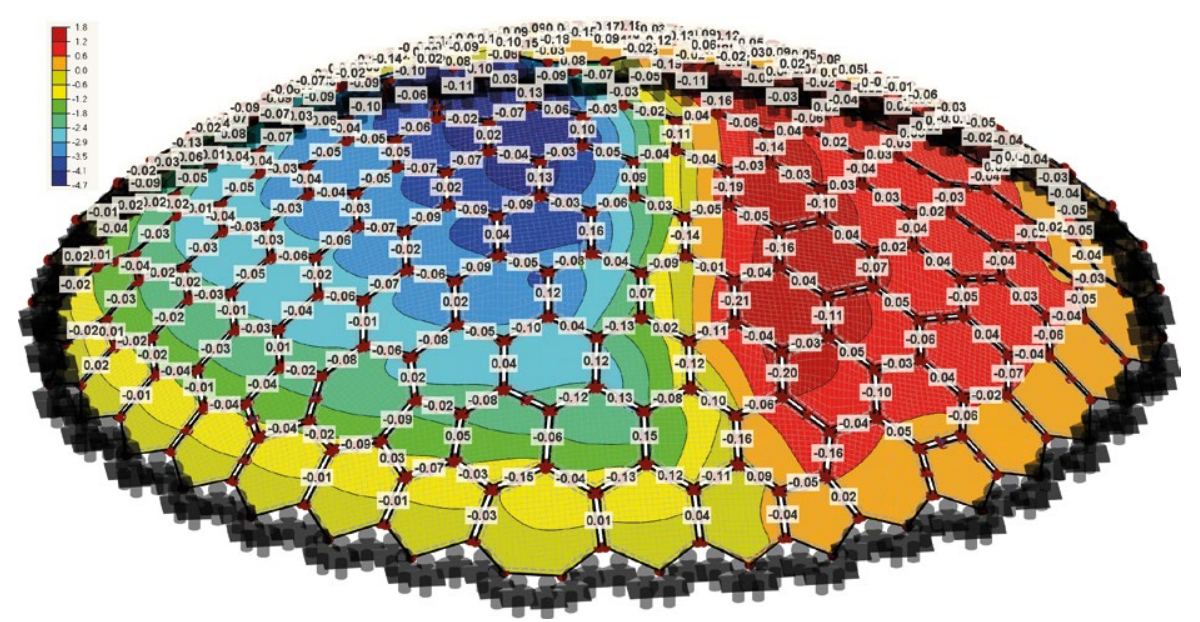

Acknowledgements

This research was supported by X-Fix GmbH, HOKU OG CNC Fertigung and
Hasslacher Norica Timber GmbH.

Bibliography

Bagger, A., 2010, 'Plate Shell Structures of Glass: Studies Leading to Guidelines for Structural Design', Ph.D. Thesis, Technic

Krieg, O.D., Schwinn, T., Menges, A. Jian-Min, L. and Knippers, J., 2015 Shells' in Advances in Architecturat

Geometry 2014 (conference paper)
Kübler, W., 2014, 'Das Neue Elefantenhaus $\operatorname{im} Z$ Zoo

Li, J.M. and Knippers, J., 2015, 'Segmental Timber Plate Shell for the Landesgartenschau Exhibition Hall in Journal of Space Structures, Vol. 30, No. 2 p.123-139.

Manahl, M. and Wiltsche, A. 2012 "“Kobra" aus Brettsperrholz: Neue Methoden zur Realisierung von Freiformfflächen aus ebenen Elementen an Prototyp erprobt;
Robeller, C., Helm, V., Thoma, A., Gramazio,

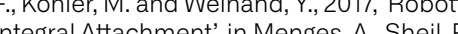
Glynn, R., Skavara, M., FABRICATE: Rethinking Design and Construction, 2017, Robeller, C., Konaković, M., Dedijer, M., Layered Timber Plate Shell', in Internation Journal of Space

Scheurer, F., 2008, "Digitaler Holzbau: (1) www.forum-holzbau.com/pdf/ihf08_forum scheurer_f.pdf (accessed 9 February 2020) 


\section{Robots for Skill Digitisation}

Johannes Braumann

Creative Robotics UfG Linz, Robots in Architecture

\section{Robots in Architecture}

In the past decade, robotic fabrication has rapidly become a relevant field of research within architecture, and is now increasingly investigated for commercial applications by both innovative startups and establishe companies. A key step toward that development has been the evolving mindset that logic and algorithms can also constitute design. While high-end architects have been using these strategies for many years, accessible visual programming tools like Generative Components, Explicit History and Grasshopper have opened up parametric and generative design to a much wider ran of users. The resulting ecosystem in which developers create plugins and add-ons for these environments has extended the software's scope beyond architecture the combination of generative design with robot simulation and code ceneration has enabled the automated and efficient fabrication of elements with small lot sizes.

Innovation for the Skilled Crafts and Trades

This area of small-scale fabrication with a high degree of customisation was the exclusive domain of the skilled of customisation was the exclusive domain of the skilled
crafts and trades for centuries, but has been increasingly crafts and trades for centuries, but has been increasis Robotics are considered a promising industry through which we can bring fabrication back into Europe. This which we can bring fabrication back into Europe. This take charge of fabrication, but that crafts and trades have the possibility to innovate and modernise by adapting with their deep knowledge of processes and materials.
However, automation and digitisation have mostly and have been medium- and large-scale companies, and trades. Some professions have already entirely disappeared, and traditional knowledge is being lost. The reason for the lack of digitisation is not primarily the cost of hardware, but the need for specialised software to control specified tasks. Cross-disciplinary efforts have also been hindered by the lack of clear task definitions, as skills are mostly passed down orally. We therefore consider digitisation and robotics in combination with accessible software not just as an efficiency-enhancing tool for craftspersons, but also as a way to capture and preserve craft knowledge and cultural heritage.

Previous Research

As part of the AROSU (Artistic Robotic Surface Processing of Stone) research project funded by the European Union - in collaboration with several partner institutions - we have investigated the mal partner istution process of stone structuring. As there is hardly any partner TU Dortmund had to carefully measure the partner TU Dortmung has to carefuly measure the poces roces, as well as the spatial movement measurem (Sten hagen et al, 2016), with high end measurement equipment. This analysis data was then used to in form the design of customised dobotic tool as well as the robot's parametric tool path layout, providing a live visualisation of the predicted groove made by the tool, the process time and approximate wear. Grasshopper was chosen as the interface for that project due to its immediate feedback and usability, with our in-house developed software KUKAlpre providing
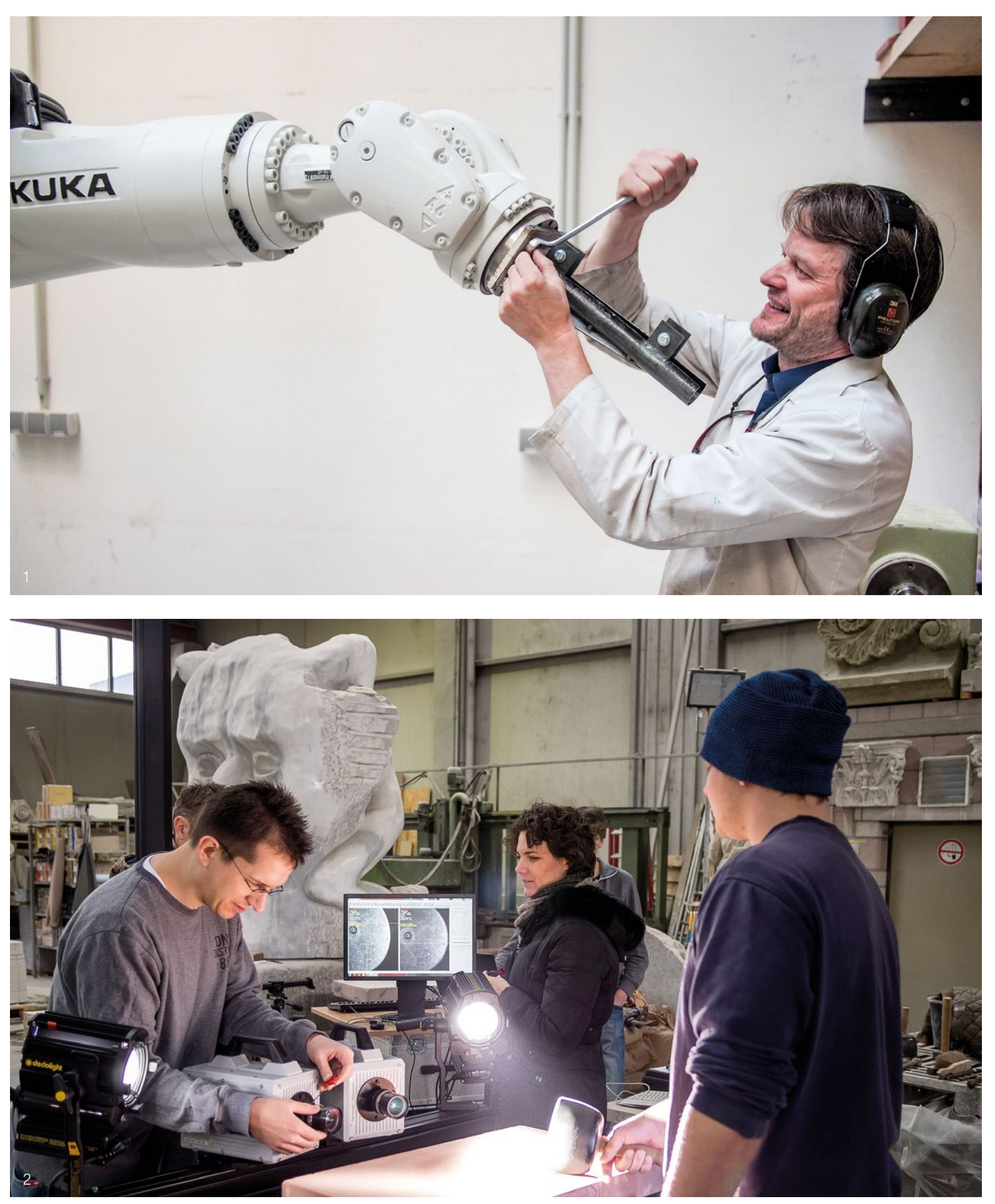

Research into robotic craft processes

2. Research into robotic craft processes. 

sical robot.

Through the Robotic Woodcraft research project (Hornung and Braumann, 2015), we introduced a robotic arm into the previous (mostly manual) carpentry workshop at the University of Applied Arts Vienna. During the three-year project runtime, the robot was used for numerous high-profile projects, both artistic and commercial in scope (figs $1 \& 2$ ), educating numerous students in robotic fabrication and enabling them to realise elaborate designs. While the establish makers provided crucial feedback, and by the end of the project could advise on reachability and other machinic constraints in robotics projects, the availabilty of highly skilled computational designers prevented them from actually taking charge of developing their processes and they remained in project management roles instead.

While both projects achieved their goals and realised a significant knowledge transfer between makers and roboticists, there was still a strong division between the disciplines, with the latter professionals providing tools and interfaces to the makers, which they could then utilise for their own purposes. This process will not change in the immediate future, as it makes sense that people specialising in certain fields can gain a deeper understanding than generalists. It is important, therefore, to provide interfaces with more flexibility and openness, so that the craftspeople cannot only adjust specific parameters but can turn their craft knowledge into code and robotic processes.

An important step in that direction has been the development of accessible parametric programmins software over the past ten years, which built upon the Grasshoppervisual programming environment while most other robotics software at the time was either standalone or integrated into Computer Aided Manufacturing (CAM) software. This made it largely application-independent enabling the user to create toolpaths for milling 3D printing weaving etc with the same software tool Today our software KUKAlpre (Braumann al, 2011) is used at over a hundred universities as well as by innovative, industry-load universiles as well as by innovative, industry-leading companies in

We believe that having accessible tools and interfaces is crucial in fostering innovation, and that innovation can best happen from within. In architecture, we are now seeing companies that automate manual technologies, such as ROB Technologies with bricklaying (Bonwetsch et al., 2016); Odico with fabricating formwork (Sendergaard and Feringa, 2016); and $m \times 3 D$ with turning regular welding into an additive construction process. These companies demonstrate that there is demand for such processes, and that smaller companies can have an
While smaller fields like the skilled crafts may not turn over a total volume approaching the trillions of Euros of the construction industy, her is also significanty less competition and small companies can complety revolutionise a field with innovative processes.

\section{Research Environment}

The global goal of making robots accessible to the creative industry is pursued by the Association for Robots in Architecture. The goal of the research department Creative Robotics (CR) at UfG Linz in Austria, however, is to have an impact on a regional scale (Braumann et al., 2017).

A key challenge for collaborations between universities and small companies or workshops is to make the initial contact, as only a few small companies actively approach academic institutions with research proposals. The

reasons for that are manifold, from concerns regarding costs to preconceptions regarding the actual usability of academic research. Most importantly, they may not even be aware of the potential of technologies such as robotics within their field. For that reason, we are working closely with two local partners. Firstly, the Ars Electronica Center in Linz, Austria, is one of the leading institutions in the field of digital arts, and as the 'museum of the future' is one of the city's main attractions with nearly 200,000 visitors every year. One focus of their work is to make technology and science attractive to young people, while also engaging their parents. There is a $\mathrm{CR}$ exhibition every year on the ground floor of the AEC inviting internationally renowned institutions such as the Institute for Computational Design (ICD) of the University of Stuttgart, the Centre for Information Technology and Architecture (CITA), Individualised Production at RWTH Aachen University, the Institute for Advanced Architecture of Catalonia (IAAC) and others to showcase their work. These e (IAAC) and often inspired visitors to share their ideas with us on how robots may be used within their own fields.

The second local partner is the Grand Garage $3000 \mathrm{~m} 2$ ' 'innoution workshop' funded by the non-profit Future Wings Foundation, recenty setup (a) creative companies. The Grand Gara is equip and creative companies. The Grand Garage is equipped 3D printers, five-axis CNC machines, metas and SLS 3D priters, five-axis CNC machines, measuning devices, etc. for companies that want pe form research and product developmentwithout having to purchase those public-private partnership, CR was provided with a large public-private partnership, CR was provided with a large lab-space within the Grand Garage, in exchange for knowledge transfer and inspiration for its robotics ab

Through these and other efforts, we have been able otherwise not have approached a research institution and have realised a number of prototypical projects, described in the following case studies. These range from small but innovative craft businesses that crea high-end products for end-customers, to a smal enterprise that is among just a few companies worldwide capable of performing high-accuracy polishing for the automotive industry. Finally, we explore an experiment non-commercial project that 1:1 matches motioncaptured movement to a robot.

\section{Case Study: Saddle Buildin}

Niedersüß is one of the last saddle-making companies in Austria. A small company of around 25 employees, it ships most of its high-quality products to internationa customers, but also supplies well-known nationa institutions like the Spanish Riding School in Vienna. Niedersüß approached us with the idea of investigating the potential for digitalisation and automation, as qualified staff were increasingly hard to find, especially when their tasks included repetitive and injury-prone work. As part of a preliminary study, we analysed their internal production workflows regarding the potentia of digitisation and robotics (fig.3).
After collecting data onsite, we came to the collusion that machines could be used for several purposes, from the CNC fabrication of the saddle core to the trimming of foam and leather-cutting. While machines can increase productivity, customers do, however, prefer a handmade leather finish on their saddles, machine-made products are perceived to have less worth and would consequently be lower in cost. The production would not, however, increase significantly as only so many saddles can be finished by the makers once the cores are milled by a machine. At the same time, automating the entire workflow does not align with the goals and image of the company. Niedersuß consequently decided to invest in robotic arms rather than a CNC machine, as these provided them with the flexibility to introduce a single new and affordable machine to the saddle-making process that is not limited to a single part of the workflow. Just by changing the robot's tools, it can perform 3D milling with a spindle one day and cut leather with an ultrasonic cutter the next. Introducing such a setup would usually require them to hire a robot integrator for configuring the machine, and possibly a software company to create the software, but they were able to set up a used $150 \mathrm{~kg}$
KISSE

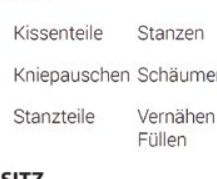

Sattellauum Schăun

Sattelistzetile Stanzen

Sattelbaum Zurichter

Satteltaschen Stanzen
vernahen

Gurtstrippen Stanzen 


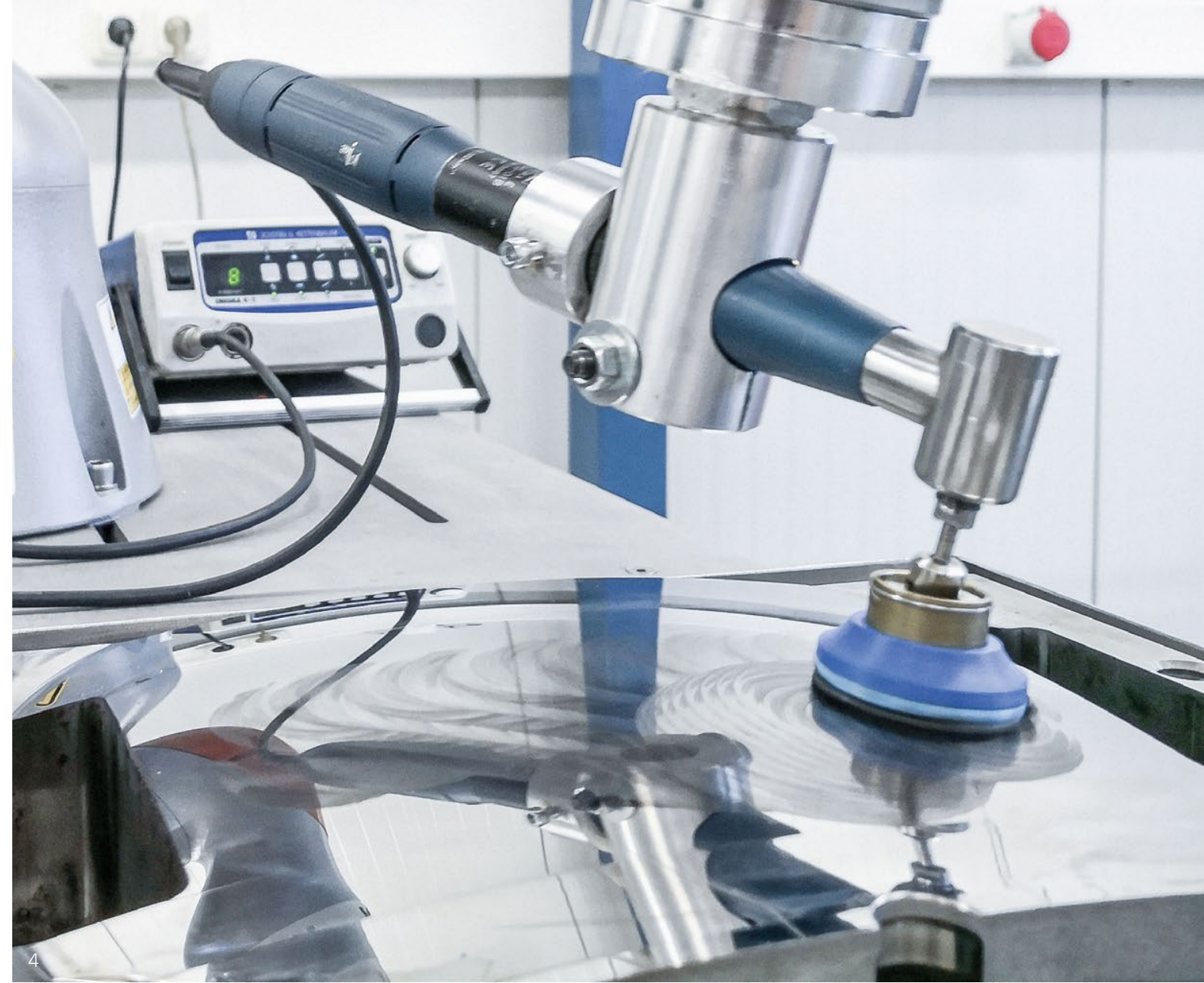

payload robot by themselves and control its processes using Grasshopper and KUKAlprc. By limiting the initial investment, they do not have to decide between machinic and manual labour but can gradually introduce the robot and continually educate their staff so that more repetitive processes may eventually be taken over.

As part of this research project, we developed an

initial, parametric model of the saddle-code geometry, making it possible to adjust it according to the individu requirements of the rider and animal. Previously, an aluminium mould had to be fabricated for every saddle, limited to just a few pre-set sizes, similar to the commercial fashion industry. Now, a standard offset model is cast in PU foam using an in-house foaming machine, so that the robot does not need to cut the model out of a single block of foam but instead removes only a few centimetres of material.

Case Study: Metal Polishing Using a Collaborative Robot A limitation of most current industrial robots is that they do not have a 'soft touch', and instead move to their programmed position without any regard to external forces. While this is a desired behaviour for many robotic applications, it also limits their applicability for processes that involve material tolerances or soft materials. Forcetorque sensors can be added to these machines, but they are comparably hard to integrate as their output needs to be calibrated, filtered and processed in order to be usable. With the LBR iiwa, KUKA developed a robot with integrated torque sensors in each axis. These sensors were primarily put there to make the robot safe to work around, as it would feel resistance not just at the flange but at all robotic joints, and be able to react to those stimuli.

We were approached by a surface-finishing company in Upper Austria that polishes CNC-milled metal parts for the automotive industry to micrometre smoothness. To do so, a polishing tool needs to be moved with a constant force in a resular way, so that material is removed evenly. Each polishing extends over many perses, which have to be different from oach other passes, which ho per this high degree of complexity that cannot easily performed by standard CNC machines and CAM software, he company has so far used machines Whil theasuing, not for the polishing process itself. Well doc process of using a robotic arm for polishing is well documented in literature (e.g. Tam et al., 1999) and actively used in industry, existing ready-to-use applications did not fulit the requirements of the company, as it wanted to direclly replicate the existing proven process without having to acqu chemicals (fig.4).

A large order by an automotive customer had the company approach us to look into developing a polish process for a freeformed surface, within a compact timeframe of a few months. As such, we decided that not be sufficient to integrate force sens or a reliable manner. Instead, we looked to the LBR iiwa as a platform with the necessary force sensitivity already mbedded within its software and hardware. Due to this integration, we were able to rapidly deploy a prototype with a robot-mounted manual tool and a constant

control force applied in negative $Z$ (vertical) direction. Thanks to the liwa's ease of use, the main challenge was, therefore, not so much in the hardware as in the software. We had to identify an ideally low number of parameters for the path planning that would change the process in a meaningful way, while ensuring that each part could be fabricated. Ultimately, just the polishing direction and three parameters affecting the layout of the spiralling tool path were chosen. A particular challenge was the handling of the edge condition, where we had to find a way for the tool to remain on top of the polished surface - as the control force would otherwise cause it to drop - while keeping the spiralling path as even as possible.

Initial testing showed that the LBR Iiwa was only able to blend motions with a point distance of $4 \mathrm{~mm}$ or more, so that very dense toolpaths caused the robot to stutter and move in a non-regular and non-repetitive way. We were able to solve that by splitting the spiral toolpath into a much smaller series of circular movements. Interestingly, the total size of the XML file generated by KUKAlprc did not seem to have a measurable impact on the robot's performance - only local density proved to be problematic.

The company has since integrated the robot into its workflow, having it mostly process regular freeform surfaces, so that its workers can focus on complex surfaces where the effort of programming and testing would exceed the benefit of the machine. As an added benefit, the collaborative LBR iiwa robot also allows the company to reduce the complexity of the safety installation, offsetting its higher price compared to other machines.

\section{Case Study: Direct Skill Transfer}

The Direct Skill Transfer project differs from the other case studies in that it is not a commercial research project but constitutes art-based research in

Ars Electronica FutureLab.

(the permanent exhibition at the Ars

Electronica Center, the exhibition concept planned for a robot installation playing a marionette (figs $5 \& 6$ ). In practice, that proved to be a significant challenge, as robots are usually programmed in relation to geometric space and speed but never to time. A robot may move to a position with a programmed speed, and it may move synchronously to another machine, but we cannot programme it to be a a given position at a given 
186
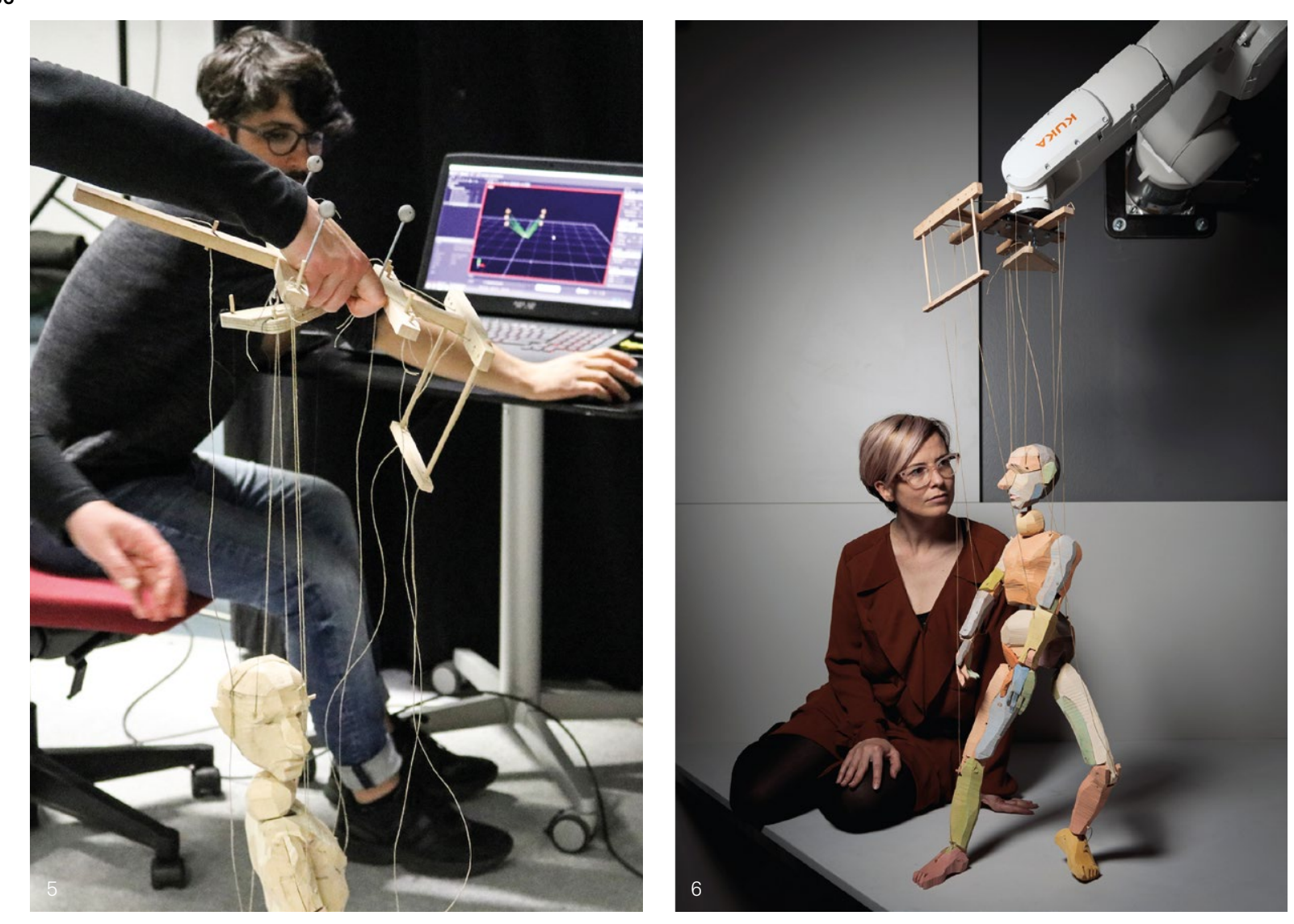

timeframe of two months, we decided to directly capture the performance of a professional puppeteer and transfer it to the robot. Using an in-house OptiTrack motion capture system, three markers were placed on the puppet's cross-bar, generating a text file that consisted of each marker's position in 3D space, as well as the precise timestamp when that position was captured. We then interpolated the data to receive one position every millisecond, rather than at the tracking system's $100 \mathrm{~Hz}$. Using KUKAlprc, the robot and toolpath were placed on the virtual stage and using its inverse kinematics solver, we cal sulted the axis position for each millisecond and wrote it into a file.

On the robot, we installed the KUKA.RSI (Realtime On the robot, we installed the KUKA.RSI (Realtme Sensor lo controller, requing an offet position at the cycle time of four millseconds. Relably sending a data package every few $\mathrm{mill}$. conds cannot be achieved with a regular Whndows PC, so we programmed a Beck hof PLC to read the previously-generated file and stream it with perfect timing to the robot, which would then duplicate the puppeteer's movement. As there are few professional puppeteers practising these days, we do not consider making the craft more readily available to the public.

\section{Conclusion: Skill Digitisation}

Based on our experience with these and other projects we see three steps in regards to skill digitisation from human to machine:

- Imitation

- Augmentation

- Autonomy

Industrial applications usually focus on imitation, where we take a given process and try to perform it with a robot rather than a person. While some processes are easy to imitate, other crafts such as puppeteering are much

more difficult.

Our main interest lies in the second step, augmentation, where we look into the value that a machine can contribute to a process that goes beyond what is possible on a human scale. For example, with the AROSU project we were able to generate non-regular chiselling patterns, while for the saddle project we enabled highly customisable saddle cores at nearly the same efficiency as identical cores. The third step, autonomy, is significantly harder to achieve as it requires the machine to learn and improve by itself. While machine learning is making huge strides at the moment, the main challenge is the quality of data required by the machine, e.g. the exact force of the chiselling movement or the 3D data of the impact, and processing that large amount of data in a meaningful way. As such, the greatest potential in developing 'augmentation' processes is to not just duplicate manual labour but find alternative ways to offer new products through machinic processes. To enable even better customisation we are working on new software solutions that enable a direct and o streaming of complex toolpaths to the robot, as well as porting to new platforms, from Autodesk Dynamo to The Cloud, where it can be integrated into innovative workflows that directly link the customer's input parameters with robotic fabrication.

It is hoped that robots combined with accessible

ftware interfaces can have a similar imp software intertaces can have a similar impact within the sklled crats and carchitecture, leading to innovations that benefithe entire community
Acknowledgements

ducted in collaboration Whith multiple partners and institutions. Bamberger Natursteinmerksistero Automation, TU Dortmund, Labo Gibson Tools, and Robots in Architecture. Contributors to the saddle project were with Maria Smigielska contributing to the metal polishing case study. The DST project team consisted of Amir Bastan Peter Freudling Stefan Mittelböotics, Erwin Reitböck from the FutureLab, and Katharina Halus as puppeteer. We are grateful for the support of

Bibliography

Bonwetsch, T., Willmann, J., Gramazio, F. Towards a New Paradigm of the Automatic, in Worre Foged, I. (ed.) 2016, Bricks /

Braumann, J. and Brell-Cokcan, S., 2011, Parametric Robot Control: Integrated CAD of ACADIA 2011 Conference, Banff.

Braumann, J., Brell-Cokcan, S. and Stumm, and Witt, A (eds) 2017 Totics, in Daas, M Architecture, Novato CA, ORO Editions.

Hornung, P. and Braumann, J., 2015, 'Robotic Woodcraft',

Steinhagen, G., Braumann, J., Brüninghaus (K) Robotic Artistic Stone Surface Production: RoblArch 2016: Robotic Fabrication in Architecture, Art and Design, Sydney. doi:
10.1007/978-3-319-26378-6.99

Sondergaard, A. and Feringa, J., 2016, Robotic Hot-Blade Culting, Roblarch 2016 Design, Sydney. doi: 10.1007/978-3-31926378-6_11

Tam, H., Chi-hang Lui, O. and C.K. Mok,

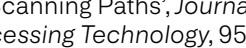


Research Summary

Building with Earth and Drones

Stephanie Chaltiel

Industrial Partners: Cloud 9, Rippmann Oesterle Knauss GmbH

Academic Institution: Institute for Advanced Architecture of Catalonia (IAAC)

Universitat Politècnica de Catalunya

Bioshotcrete is a new technology based on dronespraying clay mixes onto light formworks, enabling the development of onsite fabrication protocols at large scale. Bioshotcrete is being developed through the Innochain network by Stephanie Chaltiel and a multidisciplinary team of architects, engineers and drone experts to offer highly sustainable and affordable architectural envelopes at the intersection between shotcrete, wattle and daub. The drone spray eases the deposition of mortars in high or difficult-to-coat areas - including extreme geographies such as cliffs - without the need for labour-intensive, bespoke scaffoldings. An embedded Artificial Intelligence system - already being developed by machine learning experts - controls crack recognition, temperature mapping variable thickness and also, automated flights. It is predicted that such techniques will in the future support the development of affordabi ber possibilities. Furthermore, the unique ergonomics of the custom-made Bioshotcrete drone fitted with an adjusted Euromar pump and nozzles enablecarefully formulated

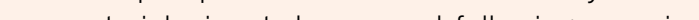
raw sequence of to borition whered foch mortar and drying Several projects were designed and constucted as Several projects were designed and constructed as prototypes at pavilion scale, hivestigating, in great detail, 'mud shells' or freeform, self-standing, compressiononly vault structures and the techniques required to implement drone-spraying construction protocols. As a continuation of the initial prototypes, research and physical tests are being undertaken to help improve existing façade refurbishments, providing drone-spraying of both wet and dry matter, and using local raw materials to provide high-inertia insulation and affordable finishings.

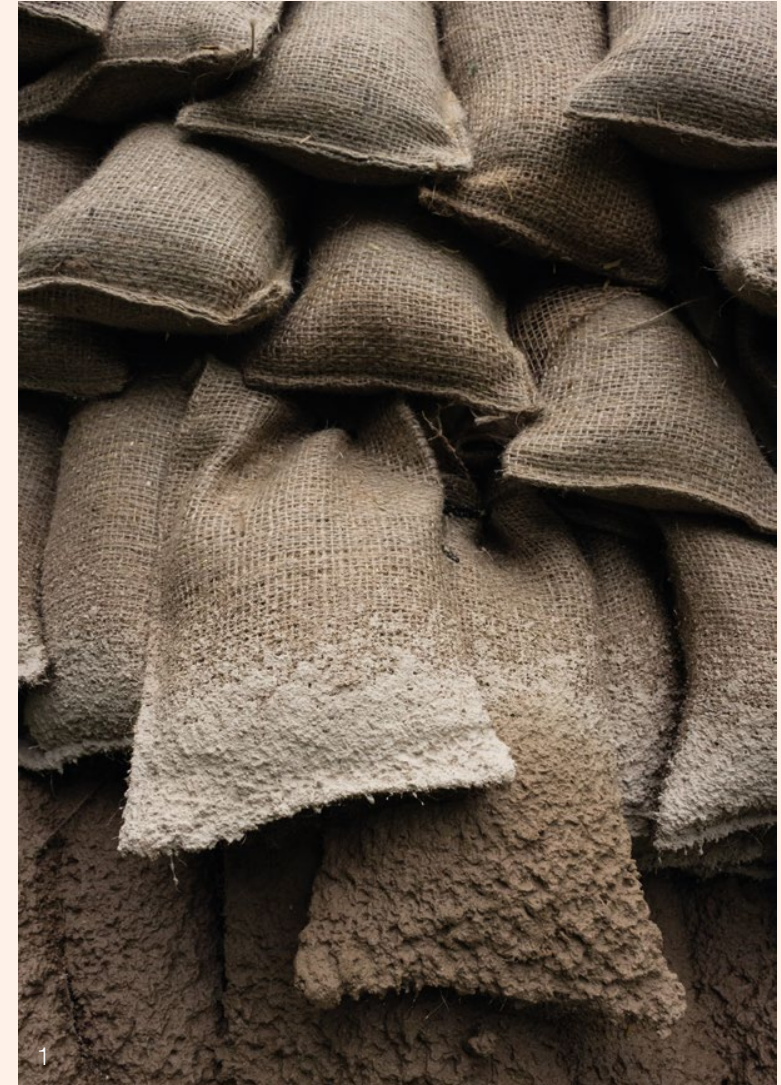

1.Jute bags filled with straw are arranged in a geodesic dome Mud Shell project, London Design Festival 2018 .

2. 'Future Earthen Dwellers' workshop (2019) at Domeaine
du Boisbuchet, where the drone spray technique was first implemented in a built project.

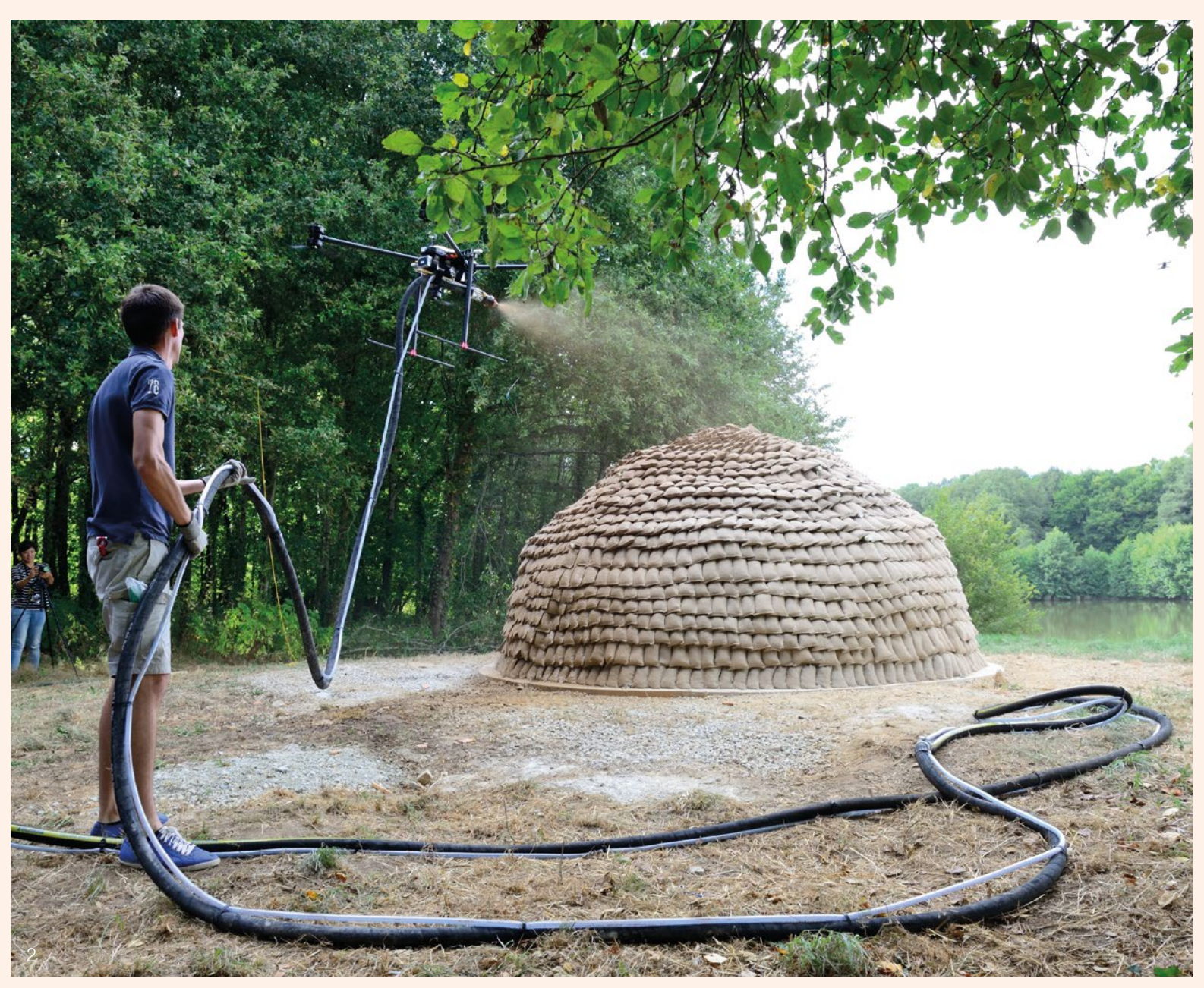

the construction of five built prototypes, ranging from 2 to $5 \mathrm{~m}$ high. An initial test was undertaken at the Barcelona Drone Center where a large inflatable dome was coated Drone Center, where a large inflatable dome was coated and cured unth sutice A DIY sprayer was fabricated for this incipient remo A DIY which the dron performed various tips. The mud done performed various trips. UK - mere subsequently shaped according to and the UK - were subsequ Centers findings. The tech when the drone wa spraying pump-hose that allowe a strong and constant Fur of material to be projected anto the light formworks. Boist Boisbuchet, London Design Festival and KADKinterior $5 \mathrm{~m}$ wall) featured prefabricated, light, dry modules arranged and fitted onto a prefabricated geodesic frame, to be rone sprayed with a smooth mortar at a later stage. Such manufacturing strategies were designed to achieve very high inertia and indoor comfort using ligh straw modules - jute bags of $15 \mathrm{~cm} \times 20 \mathrm{~cm}$, fled with one person. Blockage in the spraying phase was avoided by formulating mixes that contained only fine grains of clay and sand and thin linen fibres, which break in the spraying pump after five minutes of stiring time. The mud shells perform very well in terms of sound and wind protection. Precise numbers in such performances are being mapped and archived for future improvements, with potential for the drone spray to achieve constant not developments in the construction of large housing prototypes of $8 \mathrm{~m}$ high - designed and engineed with CanyaViva, AKT II and Summum Engineering (Salon del Mobile Milano, 2019) - and also refurbishments existing facades, where dry matter can be drone-sprayed onto wet coatings for good adhesion. This approach ends itself to fabrication of envelope insulation that does not rely on labour-intensive prefabricated panels. Tested at a height of up to $30 \mathrm{~m}$ in the drone-spray phase, uch protocols mark a turning point in the shotcrete industry and its use of sustainable, unbaked materials
in dense urban centres.
These positive emergent aspects have shaped recent 


\section{Research Summary}

Information Modelling for Assembly Planning, Sequencing and Optimisation

\section{Ayoub Lharchi}

Industrial Partners: Blumer Lehmann AG, Design-to-Production GmbH

Academic Institution: Centre for Information Technology and Architecture (CITA),

the Royal Danish Academy of Fine Arts, Schools of Architecture, Design and Conservation

While digital tools support architects, engineers and constructors in almost all aspects of design and manufacturing, their role in planning and design for the assembly of buildings remains an unexplored area. This ongoing research aims to bridge this gap by developing a general framework for the design and planning of assembly sequences within the architectural realm. Specifically, the project focuses on timber construction and aims to lay the foundations of a novel computational approach to Design for Assembly (DfA). The approach would facilitate assembly decisions in the early desion phases using integrated digital assembly models.

As a first step, this project analysed existing DfA principles in other disciplines, such as mechanical engineering and product design, and speculated on their translation to an architectural scale. By combining computer-science techniques and design practices from other disciplines, it proposed scheme through which professionls can describe, analyse and communicate assembly information. A set of computational methods and tools then provided a complete simultion of the assembly procedure and Assisted Assembly Planning (AAP) workflows, which detect any problems or clashes.

The project was carried out in close collaboration with two industrial partners with significant expertise in architectural practice, structural engineering and industrial timber manufacturing: Design-to-Production (D2P) - a design consulting firm with extensive experience in complex timber projects, which was able to contextualise the research methods and

identify real-case challenges within the timber industry - and Blumer Lehmann, a manufacturer specialised in timber construction, which brought in-depth materia and fabrication insights to the project and is embedding the outputs into existing real-world projects. The expected outcomes of this work are already beginning to bear fruit. For example, it will make a contribution to considerations of assembly in architectural design and how it can be enabled in the early phases and translated into a set of recommendations and guidelines for DfA in an architectural context. Furthermore, it presents a novel approach to information modelling in which assembly takes a central role along with other traditional embedded data. This approach has led to the development of a general framework and digital model specifications. The framework, entitled 'Assembly Information Modelling' is implemented as central digital model containing the architectural design of the structure, construction details 3D representation assembly sequences issues man assem

The research is investigating pevera ap lications, plication in various ficlds, to demonstrate the potential of proposin approch: during the assembly rrocedure second autone during the assembly procedure; second, automatic robotic assembly, whereby the assembly model is stetched to exp, whe is capacity to new applications, such as industrial obots for digtol fabication; thich collaborative assembly design, whereby cloud-AEC platforms are used to provide a common working platorm, and, finally, machine (ntion.
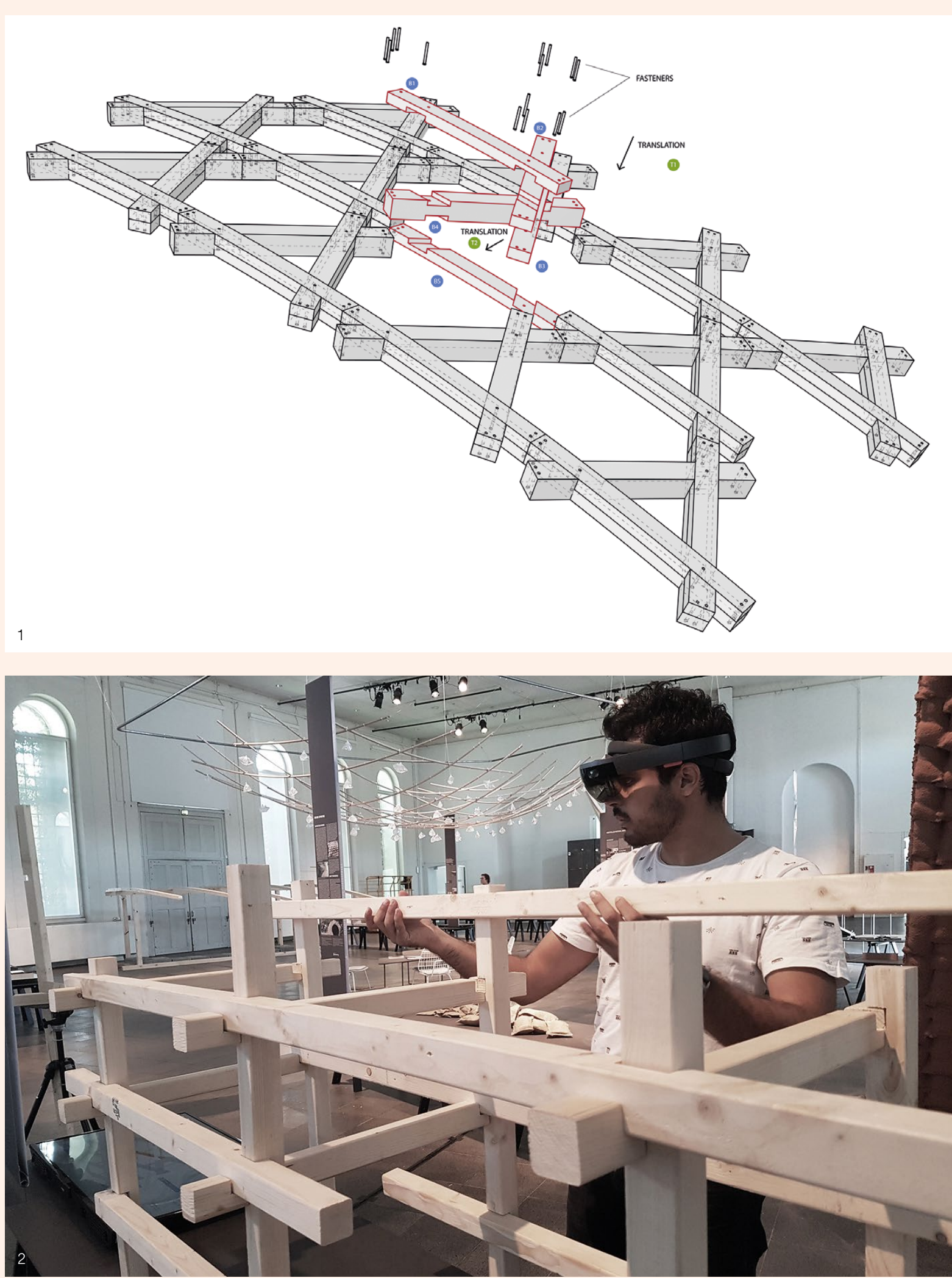

1. The model is enriched with assembly instructions and sequencing. 2. Assembly Information Modelling (AIM)
enables the assisted assembly of complex
structures without previoustraining 

for Automotive Design

Arthur Prior

Industrial Partner: Foster + Partners

Academic Institution: The Bartlett School of Architecture, UCL

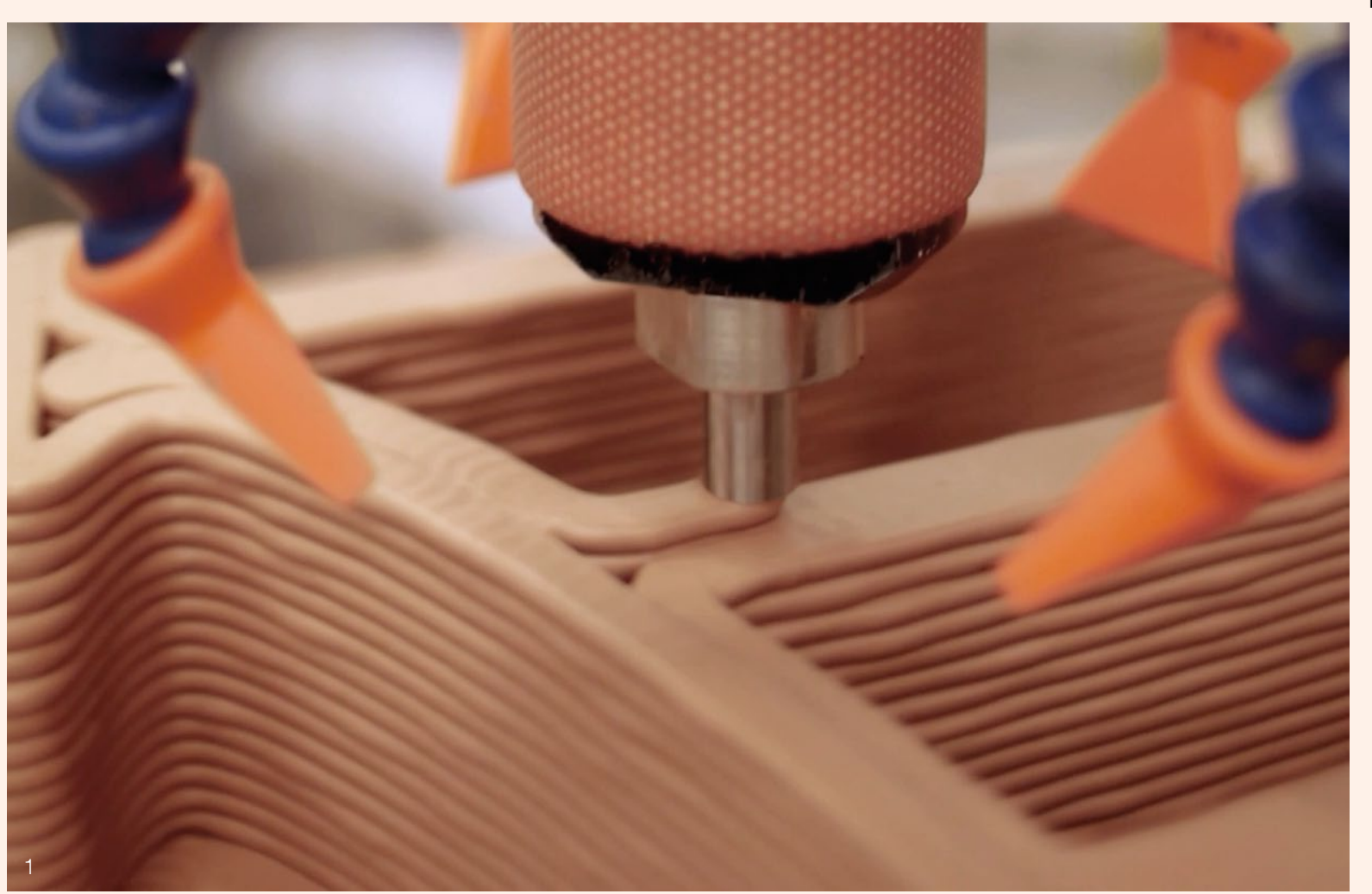

Clay modelling plays a crucial role in automotive design. It is widely acknowledged that physical sculpting results in high-quality surfaces that the end-user can see and touch. Horizontal arm-milling machines are standard clay models. To date, however, clay application remains an entirely manual process.

During model preparation, 750-900l of heated clay is applied manually to a Styrofoam armature.

This physically demanding and repetitive process

takes up to 120 hours, during which employers must balance productivity with Occupational Safety and Health (OSH) compliance such as assessment of repetitive tasks, manual-handling operation

regulations, and so on

Overcompensation occurs when trying to judge the correct thickness of clay to apply, resulting in 15\% material waste after modols are milled-out. Ford is the only automaker known to recycle clay (10\% annually).

. styling takes a minimum of 12 months before 'design 20,000 hours modelling the Ford F-150 Raptor.

There are over 200 design studios internationally: $48 \%$ in Europe, $34 \%$ in Asia, $15 \%$ in North America and 2\% in South America. Studios are particularly concentrated in areas such as Bavaria, Baden-Württemberg, California, Coventry, Turin and Shanghai. These studios consume lot of clay: $90,700 \mathrm{~kg}$ per year at the Ford Product Development Center in Dearborn (United States) and 80,000kg peryear at the Mercedes Benz Advanced Design Studio in Sindelfingen (Germany). Collectively, the industry uses 3,000 tonnes of clay per year, which is enough to create 2,400 models.

In this context, the potential for efficiency gains is significant. Seeking alternatives, automakers began investing in CGl and VR/AR/MR technologies from the 1990s.' Despite major advances, car designers have remained faithful to clay. Life-sized models are considered the most direct way of communicating design information. However, while automakers are increasingly incorporating Additive Manufacturing (AM) into design workflows, conventional additive materials (e.g. photopolymers) cannot be reshaped retrospectively, making design changes less direct and spontaneous compared to clay. The use of AM is generally limited to small-scale interior components (such as airto small-scale interior components (such as airconditioning vents and door handles). Modelling clays There are currently no solutions in the marketpla for applying clay automatically. This three-year project for applying clay automatically. This thee-year project responded to this noed by developing a solution th

manufacturing. Liauid Deposition Modelling (LDM) technology exploits the phase-change properties of modelling clays, allowing complex freeform surfaces to be printed quickly and eficiently, layer by layer. This offers a pragmatic way for designers to both utilse AM

- Provides a solution for automatically applying clay quickly and accurately.

- Makes 'lights-out' (overnight) production possible by reducing time to market.

des a way for employers to avoid manualhandling operations that involve a risk of injury.

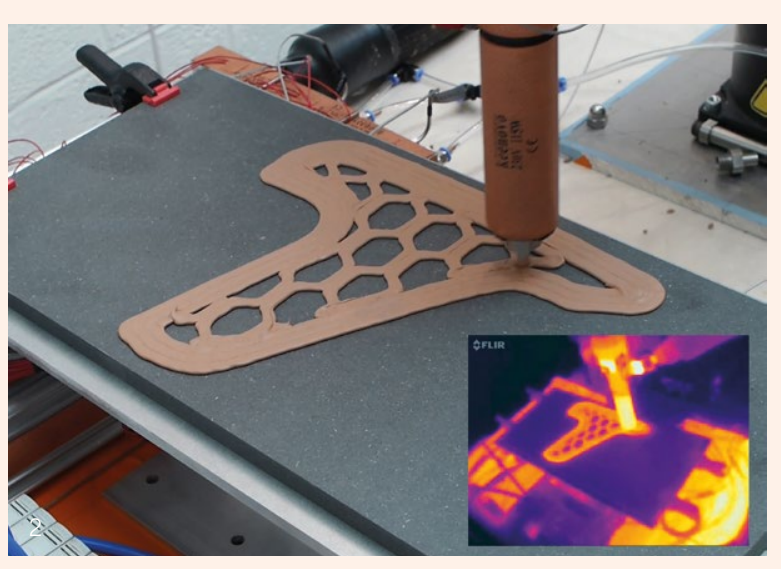

- Reduces material waste from $>15 \%$ to $5 \%$.

- Promotes the continued role of physical sculpting in automotive design.

- Interfaces with existing downstream workflows and studio equipment (e.g. milling).

- Enables design studios to further extend their engagement with digital manufacturing.

This innovation has so far been demonstrated via a small-scale proof-of-concept machine (Technology Readiness Level 3 , as per European Commission definition). A new project is currently under way in Germany to develop this technology for full-sized vehicle prototyping in a studio environment.

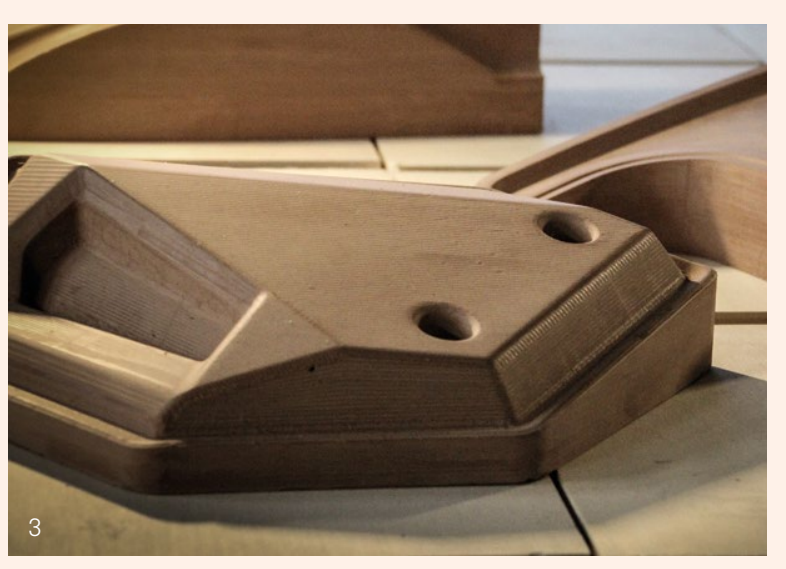

Note

1. CGI: computer-generated imagery VR: virtual reality MR: mixed reality 


\section{Research Summary}

Flectofold: From Academic Research to Architectural Application

\section{Saman Saffarian}

Industrial Partners: str.ucture GmbH, S-Form GmbH

Academic Institution: Institute of Building Structures and Structural Design (ITKE)

at the University of Stuttgart
The aim of this project was to devise a lightweight, energyconscious, materially intelligent and economically feasible solution for climate-adaptive building envelopes. (bio-inspired) product called Flectofold, which is a modular shading system capable of controlling the penetration rate of solar radiation through the building approximately $1 \mathrm{~m}^{2}$ and has an integrated pneumatic bending onto the body of the materially-graduated Fibre-Reinforced-Polymer (FRP) laminate and as Flectofold is economical in terms of fabrication and transportation, thanks to its modularity and its capability to be flat-packed. It is also lightweight and materially to be liat-pack the is to intellgentition and is efficient in terms of maintenR due to its reliance on material compliance rather than mechanical hinges. In terms of operation, it is enegyefficient thanks to the low air presure (approximgtely e.04-0.06 bar) required, and due to its capacity to store 0.04-0.06 bar required, and due to its capacity to store

\section{Research Methods}

The initial concept of the Flectofold module was created by a large collaborative team of researchers, and resulted in a proof-of-concept prototype (Körner et al., 2018). consecutive full-scale demonstrators that integrated all technologies into an architecturally detailed solution (Saffarian et al, 2019). This was crucial, not only for (Scientian et al., 2019). This was crucial, not only for These aspirations materialised in the form of a bionic envelope. Each Flectofold module covers an area of cushion. Once pressurised, the cushion induces elastic a result, generates movement and transformation. the potential resticed, and due to ts cap The Flectofold module was further developed into two contexts, but also for eventual implementation in the building and construction industry.

Three parallel strands of development were needed to move from the successful concept to a credible building-scale demonstrator piece: 1 . Development of the Flectofold laminates through a precise investigation of fibre deployment (lay-up) strategies, and constant monitoring of the resulting kinetic performance. This strand aimed to increase scale and movement efficiency, optimise cyclic performance and reduce material fatigue; 2 . Development of a suitable support system to secure the correct alignment of Flectofold modules with the underlying guiding seometry, incorporating a tilting mechanism to adjust the axial rotation of individual modules for optimal and symmetrical surface coverage, and to implement a pneumatic tubing-management system to enhance the clarity of systematic assembly and maintenance; 3. Development of a fully controllable pneumatic actuation system capable of driving each module in an individual or synchronised fashion, based on active us incer user This strand rogracis This strand requid and software (web-bas

\section{Research Partners}

A large group of industry and academic partners contributed to the development of this project. Most notably, the successful implementation of the materially graduated lamination process was achieved through an active collaboration with the Institute for Textile and
Fiber Technologies (ITFT) at the University of Stuttgart
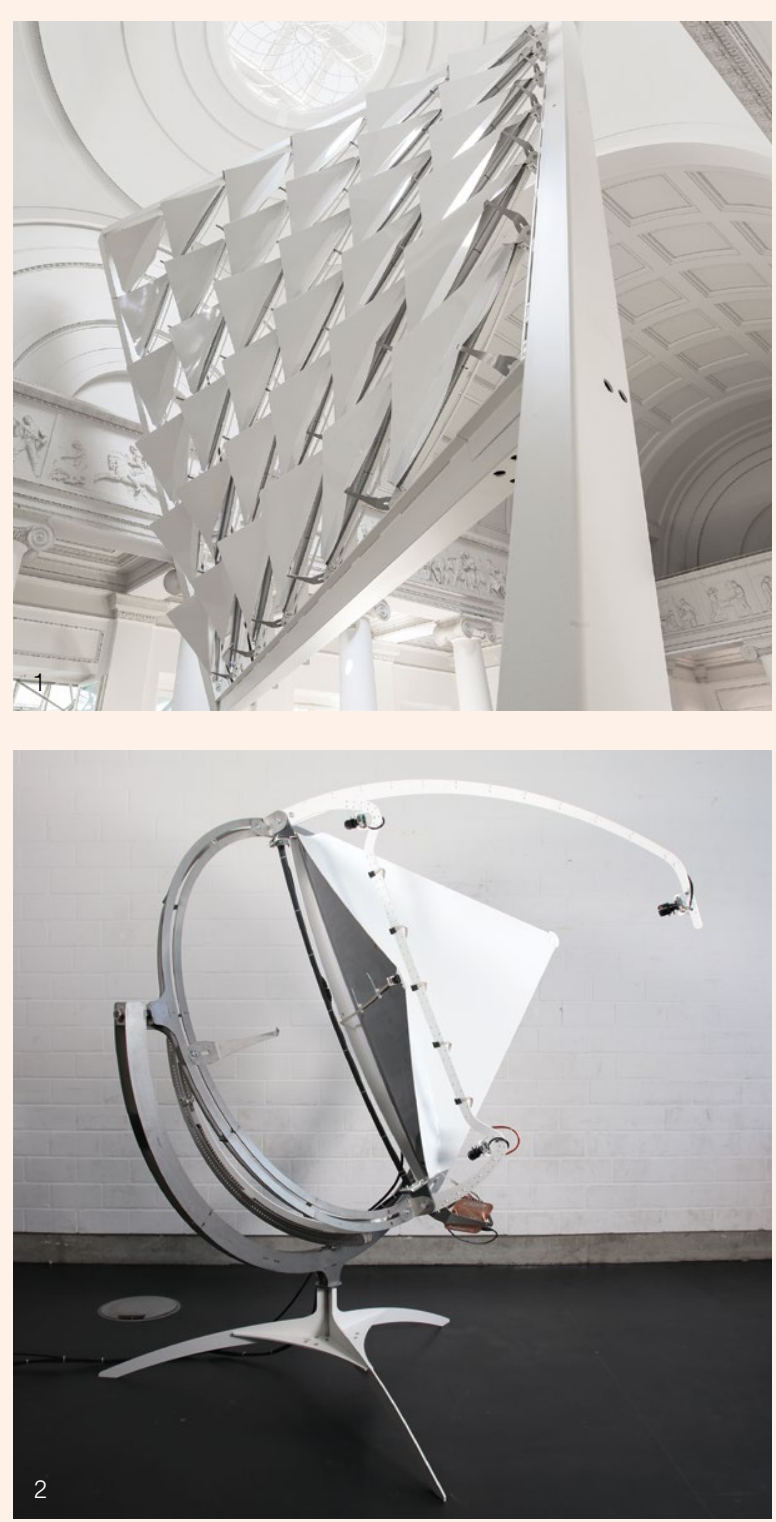

and a mutually beneficial partnership with C-Con. The pneumatic actuation and control system evolved over the course of the project into an advanced solution through an iterative prototyping process, in close collaboration with the Institute for Control Engineering of Machine Tools and Manufacturing Units (ISW) substructure required the active engagement of local steel manufacturers Roleff GmbH and Grözinger GmbH, alongside engineering colleagues at the Institute of Building Structures and Structural Design (ITKE).

Research Outcomes and Applications

During the course of the project, many proof-of-concept prototypes, probes and assemblies were designed and protonyes probes and assembles wered consequent development steps and to propel the research forward. Interim (milestone) results were showcased at various events, among which the followin cho Architektur at the Natural History Museum, Stuttgartwhich wich presented the technology to the public, using the demonstrator as a testing ground for monitoring and assessment of the long-term kinetic performance of Design for a New Materiatice Futures. Bullding Design for a New Material Age at The Royal Danish Academy of Fine Arts, Copenhagen. This latter event exhibited a revised version of the Flectofold

demonstrator as a full-scale architectural mockup together with an enhanced kinetic performancemonitoring device that collected in-depth motiontracking data, using multiple sensors and cameras, on the kinetic performance of a single Flectofold data will be utilised to enhance the design and inform the materialisation strategy of the next generation of materially-graduated FRP laminates for elastic-kinetic modules on climate-adaptive building envelopes in the near future.

\section{Bibliography}

Körner, A., Born, L., Mader, A.,Sachse, R., Saffarian, S., Westermeier, A., Poppinga, S., Bischoff, M., Gresser, G.T., Milwich, M., Speck,T. Device for Complex Free Form Famades' in Smart Materials and

Saffarian, S., Born, L., Korner, A., Mader, A., Westermeier, A., Poppinga, S., Milwich, M., Gresser, G.T., Speck, T. and Knippers, J., Facade Shading Device', in Knippers, J., Schmid, U. and Speck, T.
(eds.), 2019, Biomimetics for Architecture: Learning from Nature (first edition), Basel, Birkhauser Verlag GmbH, p.42-51. of the University of Stuttgart. The development of the module over the course of the exhibition. The collected
1. Flectofold Demonstrator I at Baubionik biologie beflügelt archititektur exhibition,
Natural History Museum, Stuttgart, 2017.

2. Flectofold kinetic performance monitoring device at Practice Futures:
Building Design for a New Material As

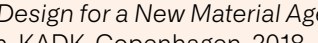




\section{Research Summary \\ Concrete Deposition: Choreographing Flow}

\section{Helena Westerlind}

Industrial Partner: White Arkitekter AB

Academic Institution: Royal Institute of Technology (KTH), Stockholm

This project investigates how additive manufacturing, supported by digital work Hlows, can inform new mater strategies for concrete in architecture. The additive process is explored to develop concrete as a medium that performs, as opposed to merely reproducing form. For this purpose, concrete deposition signifies fundamental departure from formwork-based techniques. The formal articulation of concrete-flow is no longer shaped by the constraint and control imposed by a static mould, but by the programmable motion performed by a numerically-controlled machine, turning lines into material traces. Similar to weaving. lines come together to form a surface, and in the notation of architecture, the role of the line shifts from representing the perimeter of form to constituting the path along which the material preforms. Beyond the mere stacking of material this project asks how the controlled interweaving of lines can be programmed to introduce structure and variation into a previously uniform and monolithic material.

In extrusion-based additive manufacturing an object is typically materialised through the incremen

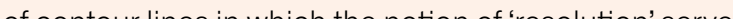
as a quantitative measure of the level of detallowich the physical object corresponds to its digital counterpart. When the additive process is scaled up, the layere structure becomes an increasingly visible feature of the surface quality of the finished object as the level of resolution typically associated with so-called $3 \mathrm{D}$ printing is not achievable within a realistic timeframe. Therefore, instead of the simple stacking of contour lines, this project set out to expand the tectonic potential of the additive process by investigating the relationship between the localgeometry of the line itself and the
The project began with studying the correlation between robotic motion and material behaviour. Formal features, such as overhang and bridging, were tested in relation to the rheological properties of various concrete mixes. The project then focused on incorporating these findings into the numerical control of continuous depositions of material. By exploring variable stacked configurations of selected line types (oscillating, looped, alternating loop), motion patterns were developed that presented new means

of controlling the density, porosity and surface articulation of the material. The ability to further control the parameters of the line in terms of frequency and amplitude made it possible to locally adapt patterns according to specific requirements. After having shown the feasibility of the approach, the project moved on to expand the selection of possible line types by weaving new lines from control points. In addition to the standard subdivision of a surface geometry into contour lines: a further discretisation of the line introduced a finite

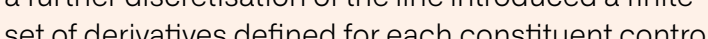
seint. From this set of points, a numerical sequence was used to reconfigure the line in an automated process that generated 6501 new line types from

The approach developed within this project demonstrates the need to re-evaluate the norm and conceptions that so far have defined concrete as a primanly monolithic matenial. By choreographing the path of deposition, variable material structures with adranced marial performance.
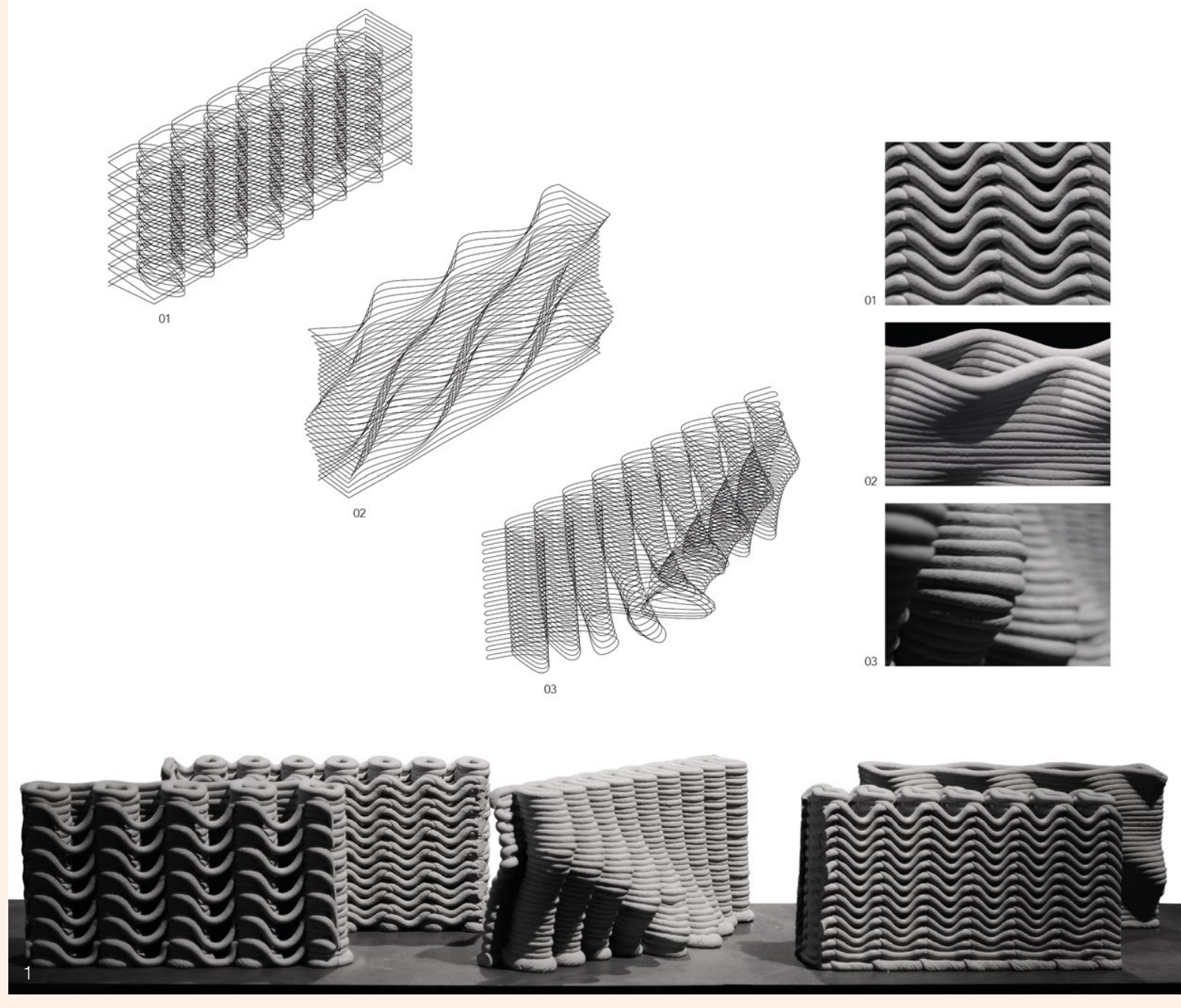
Further Perspectives

Performative Materials

and Systems, and Additive

Manufacturing Futures

Johannes Braumann

Creative Robotics UfG Linz, Robots in Architecture

Isak Worre Foged

Aalborg University

\section{Materials}

All materials perform. The level of performance is tied to objectives, processing systems and context. Water can be a fluid thermal reservoir at $15^{\circ} \mathrm{C}$; a gas-like cooler at $40^{\circ} \mathrm{C}$; or a solid casting block at $-5^{\circ} \mathrm{C}$. When subjected to environmental changes, the material undergoes a transformation of properties, which fundamentally alters its capacity to perform.

Clay, meanwhile, is a natural material found a few metres below ground. As a raw material it costs little to extract and, therefore, is economically performative. Clay is formed into bricks, fired at around $1,000^{\circ} \mathrm{C}$ to create a structural element with high compressive strength, thermal capacity, ability to sustain weathering and an articulated colouration. The firing process has a high-energy cost that from a material-processing perspective, renders clay bricks a low performance material Bricks are transported to a construction site and there they find their final position in the brickwork. The heavy weight of bricks - at $2100 \mathrm{~kg} / \mathrm{m}^{3}$ - prodwork a high transort-energycost, which equates to puces a high transort energ This aspect is, of course, related to the distance between raw-clay site, brickyard and construction site, which raw-clay site, brickyard and construction site, which then adds a materials onigin isto the equation of its buildings stand for centuries, if not millennia, significantly buildings stand for centuries, if not millennia, significantly
reducing the embodied annual energy cost of firing and performance of such buildings. Depending on the performance of such buildings. Depending on the brickwork design and constellation with oher materials, clay bricks embody thermal and humidity-regulating capacities, visual and tactile qualities and extraordinary

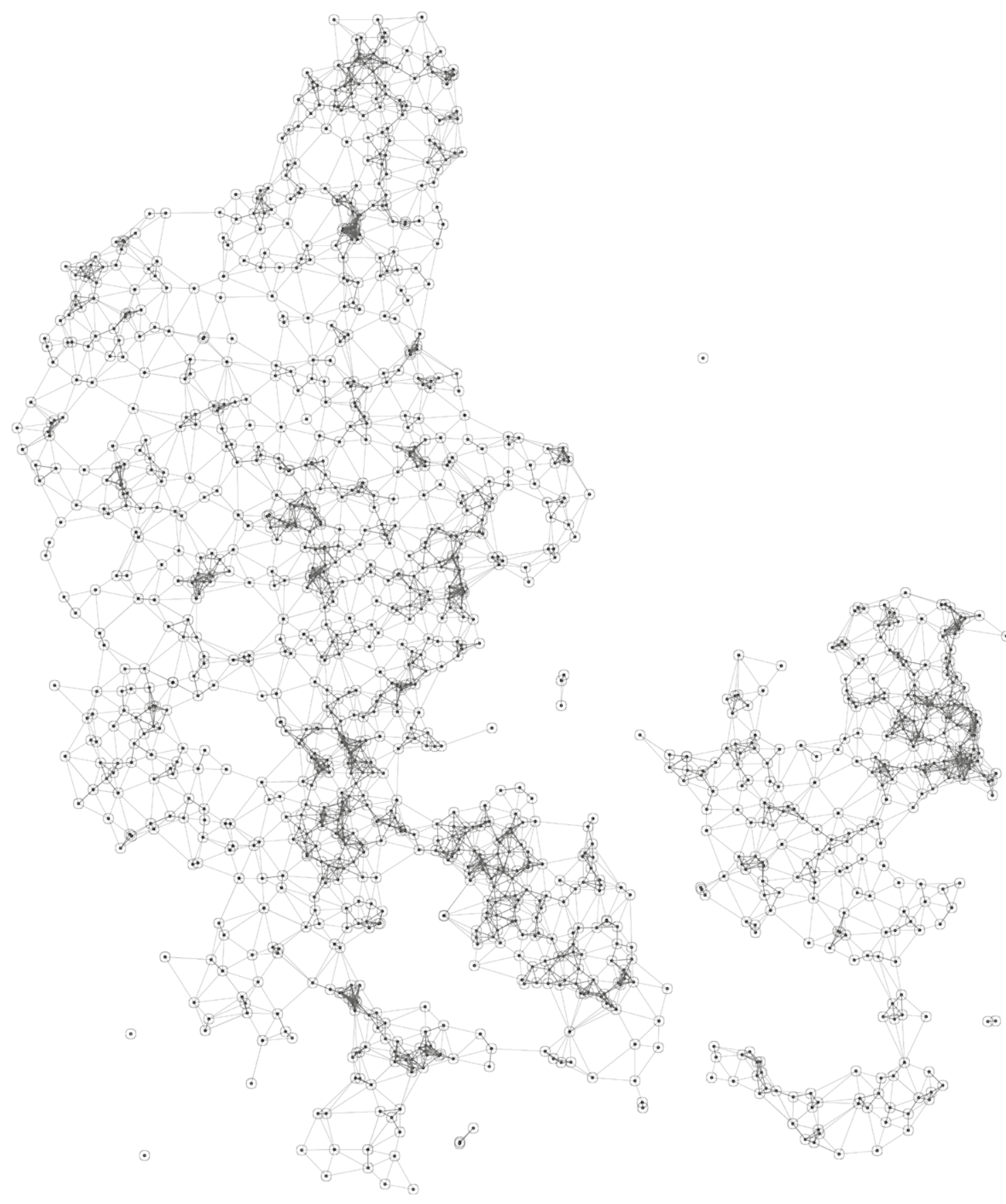




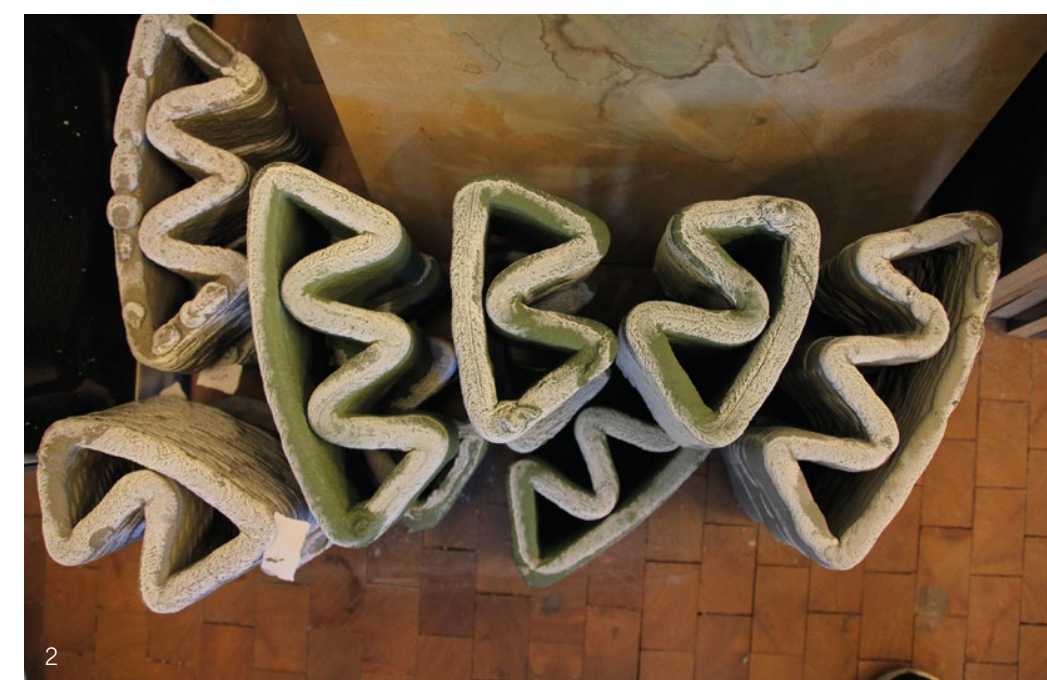

2. Robotically additive-manufactured
bricks for the Thermal Tower Project (2018) based on developing thermally-designed concrete composite made through additive MB Jensen (2018).

3. Robotically additive-manufactured Termal Tower Project (2018) in the Utzon thermally-designed concrete composite. Photo: IW Foged.

environmental and compositional advantages that, in turn, support the design and making of architectures that are treasured and maintained.

Concrete is an ancient material invented by the Romans, then forgotten, and later re-engineered in modern society. It is a composite of cement (as binder), an aggregate and water, with the ability to perform state-transition from fluid to solid through the chemical reaction of hydration. Similar to clay, cement is developed by heat processing or calcination, where the kiln is heated to $1,450^{\circ} \mathrm{C}$. Hence, from a performance metric of embodied energy, concrete shares the same problems as clay bricks. An engineered composite, concrete's structural and thermal performances can be designed and configured by inducing air to create internal pockets, which alter its density, thermal conductivity and struckura which alter its density, thermal conductivity and structy capacities. Air, therefore, becomes a performative ingedient of the composite. Similarly, when a few grams changes its thermal properties, depending on the changes its thermal properties, depending on the are maintained. When water is added and mixed with are maintained. Wha water wh ad sed and mixed with

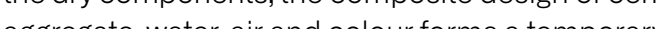
aggregate, water,

For robot-based layered additive manufacturing, the specific cement type and water conentenabling the specific cement type and water content enabling the fluid state must be procisely designed to pass easily through pumps and tubes. Aggregates must be smal and must not clusterifthe speed of material How changes, and the fuid material must be sollid enough to allow addive layers to be added successively before the final hydration process solidifies the material When all dynamic processes of the material composite interactions have taken place, the final material and form enter a combined articulated material performance. This material-form state is designed through a series of integrated processes between molecular material properties, processing techniques from kiln to robot, and the developed making hardware.

\section{Systems}

Turning a material into a product demands different types of processing, ranging from traditional craft methods such as chiselling, sawing or casting to highly automated cyber-physical systems, where production processes stretch from the digital realm into the physical space. These machinic systems enable higher productivity and efficiency at a potentially lower total cost compared to manual craftsmanship, but also greatly increase the complexity. Processes that were previously handled by a single maker may now require a team of professionals, from programmers who work with Computer-Aided Manufacturing software to application engineers, machine operators and quality assurance staff. The main costs therefore no longer relate so much to the mo costs the (astructure.

For a long time, this move toward cyber-physical systems formed a significant gap between the individual makers who relied on their own mastery of tools and malerials, and industrialised fabrication, which depends on highly accurate and expensive machinery. We can observe acchange, however, as machines become finen financial sense. That process is diven by expiring funclamentalpatents (such as in the case of 3 D printing), greater demand, growing digital literacy among potential users, and a resulting interest in

Machines are commonplace in industry, such as in the timber industry, where Computer-Numeric
Control (CNC) machines can even be found in smaller

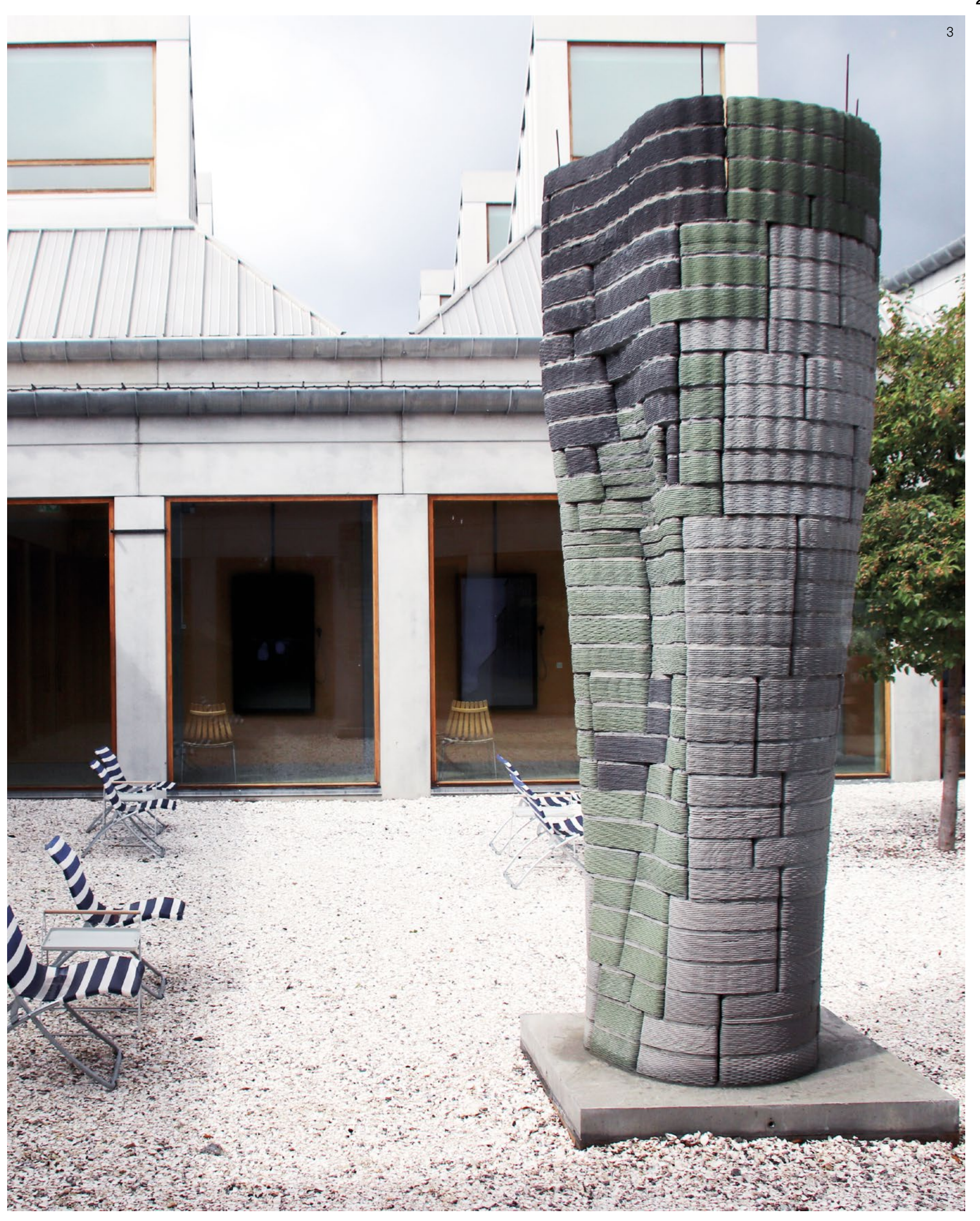


workshops. At the same time, we can observe the increasing proliferation of industrial robots, not only in experimentation-ficndy environments, such as fients thu or startups, but also within traditional fields that previously relied on manual making. The advantage of robotic arms, Compared to regular CNC machines, is that they can be equipped with a wide variety of tools to perform myriad tasks. While the timber industry's requirements are mostly fulfilled by subtractive processes that are readily available in industry, the crafts are looking for ways to transfer their manual knowledge to machines, requiring the development of new hardware and software. So-called 'cobots' (collaborative robots) offer entirely new approaches to skill digitisation, combining a robot's accuracy and speed with safety and control over the applied forces.

New machinic possibilities previously reserved for large-scale businesses and research institutions are now opening up new fields of investigation for smaller companies. Within additive manufacturing, many startups are developing potentially disruptive innovations in their respective fields, such as 3D-printed concrete in architecture, as demonstrated by XtreeE in France and Mobbot in Switzerland. While the technologies underlying these evolving processes have been around for many years, it took until recently for them to enter the market and provide services. What is particularly interesting about these developments is that they are coming from within the architectural design community, rather than from engineering. The significant challenges that these innovators are facing are not just technological but also regulatory, as the standards differentiate between regular and 3D-printed concrete. To solve these problems, the community will need to collectively engage with regulatory bodies.

\section{Futures}

While the aforementioned materials may be considered somewhat old-fashioned, especially compared to more exotic material composites, they represent a significan part of the primary building fabric and are studied in numerous ongoing architectural research projects for additive manufacturing. The design and control of the material phase-state condition (being solid, liquid or gaseous) is central to the making process, which mean that architects must understand and compose not only a material's end-state, but also its transformation processes and changing properties. Both concrete and clay are becoming favoured material substrates for additive manufacturing, precisely because they embed material performances from fluid to solid states. As familiar and tangible materials, they point to how we might describe and develop material performances for future manufacturing processes in architecture, and how these are tied to our abilities to understand complex dynamic material systems across time domains.

This future-orientated endeavour is helped by current technological developments, where machinic systems are no longer exclusive to high-end industry, but are also available to small enterprises, which account for the bulk of GDP in most countries. Innovations in making these machines accessible and affordable are, in turn, enabling startups to develop disruptive technologies, especially in the crafts field, where there is a constant demand for skilled labour. These technologies allow users to combine their in-depth knowledge of materials and manual processes with the advantages of machines - thus, not replacing labour but creating entirely new products and services. Most importantly, this innovation can happen within high-wage countries, keeping manufacturing within the region minimising brain drain and reducing emissions from long-range transport.

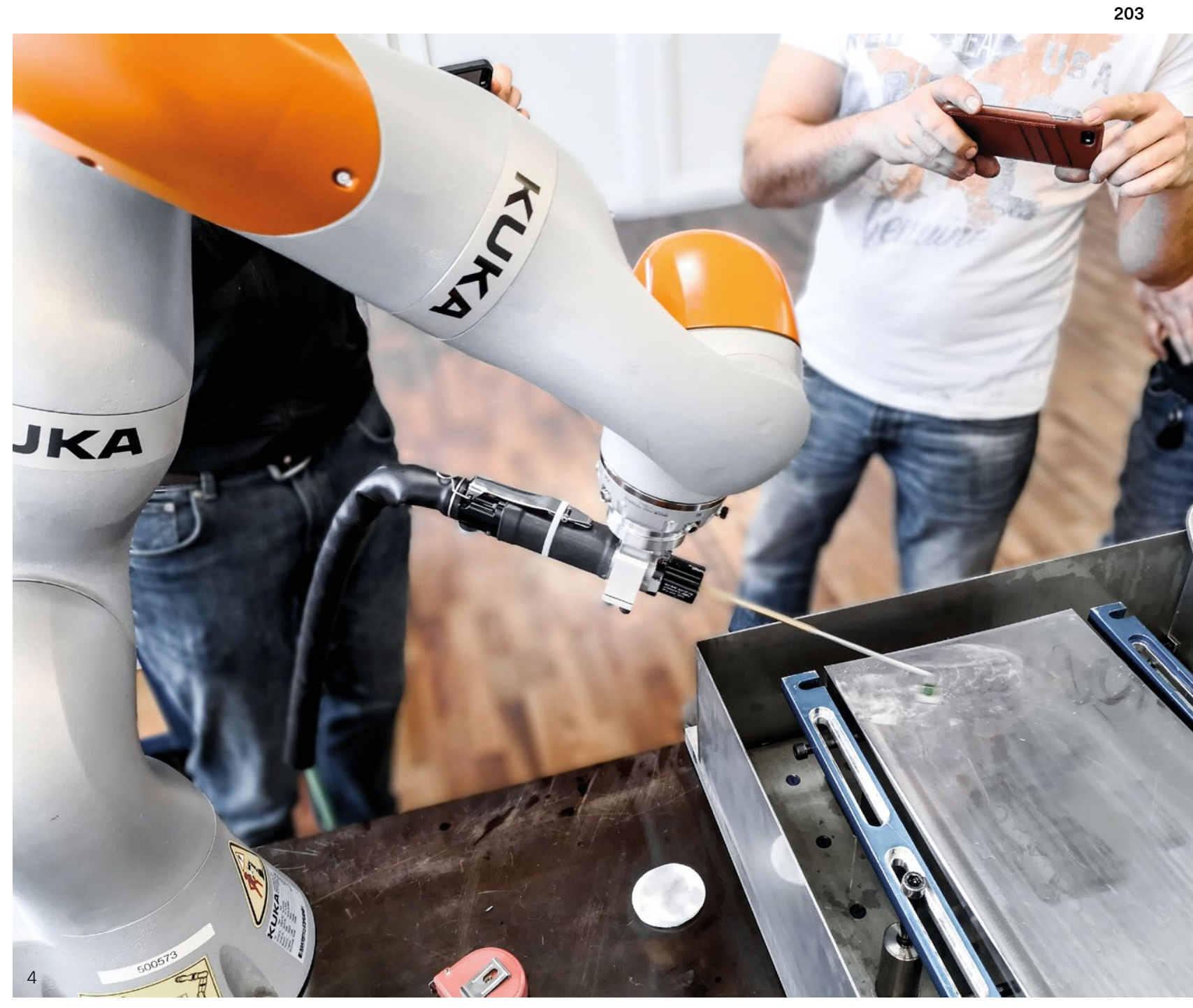




\section{Innochain: External Perspectives}

\section{Visiting Scientists:}

Mark Burry

ity of Technology, Australia

Christoph Gengnagel

Berlin University of the Arts

In dialogue with:

Anja Jonkhans

Institute of Architecture, University of Applied Arts, Vienna

Anja Jonkhans (AJ): I would like to start by asking what is the role of the Visiting Scientists and how were you engaged in Innochain?

Mark Burry (MB): To respond to presentations with our reflections on how new enquiries correlate to established research in the field, how emerging and emergent papers, presentations, exhibitions, conferences, etc. span both the science and art of the work.

AJ: You attended two Innochain colloquia some months apart, which gave you the opportunity to observe and measure rates of progress. varying approaches, and different combinations of academic and industry partne.

MB: Yes, there seemed to be a variety. Some candidates were embedded within ongoing group research projects, others were operating with greater levels of freedom, and some seemed to be seeking more constraint. Likewise, commitment from industry varied, where typically engagement relied on a key contact with a deep personal interest So one of the key challenges for Innochain, and for future similar collaborations is to key challe kind fo normation in erms of expectations of how a candide should be superised Located across five EU countries, this was inevitably a steep and complex challenge.

Christoph Gengnagel (CG): I can support this comment, because if I'm comparing the Innochain constellation with a PhD programme that we are running at the same cone at the Unvivsity of the Arts in Berlin, then I think there are several addilionalchallenges. One of the specific issues in Innochain was the very different approach of the international academic environments to executing a PhD in the UK, Austria, Spain, Germany or Denmark. Forme hese differences became, at a certain point, a greater challenge than the expected frictions between industry and academia. Here, the completely different ideas about time and speed in academic and professional practices were an issue, although, on the other hand, the importance of a project like Innochain lies in

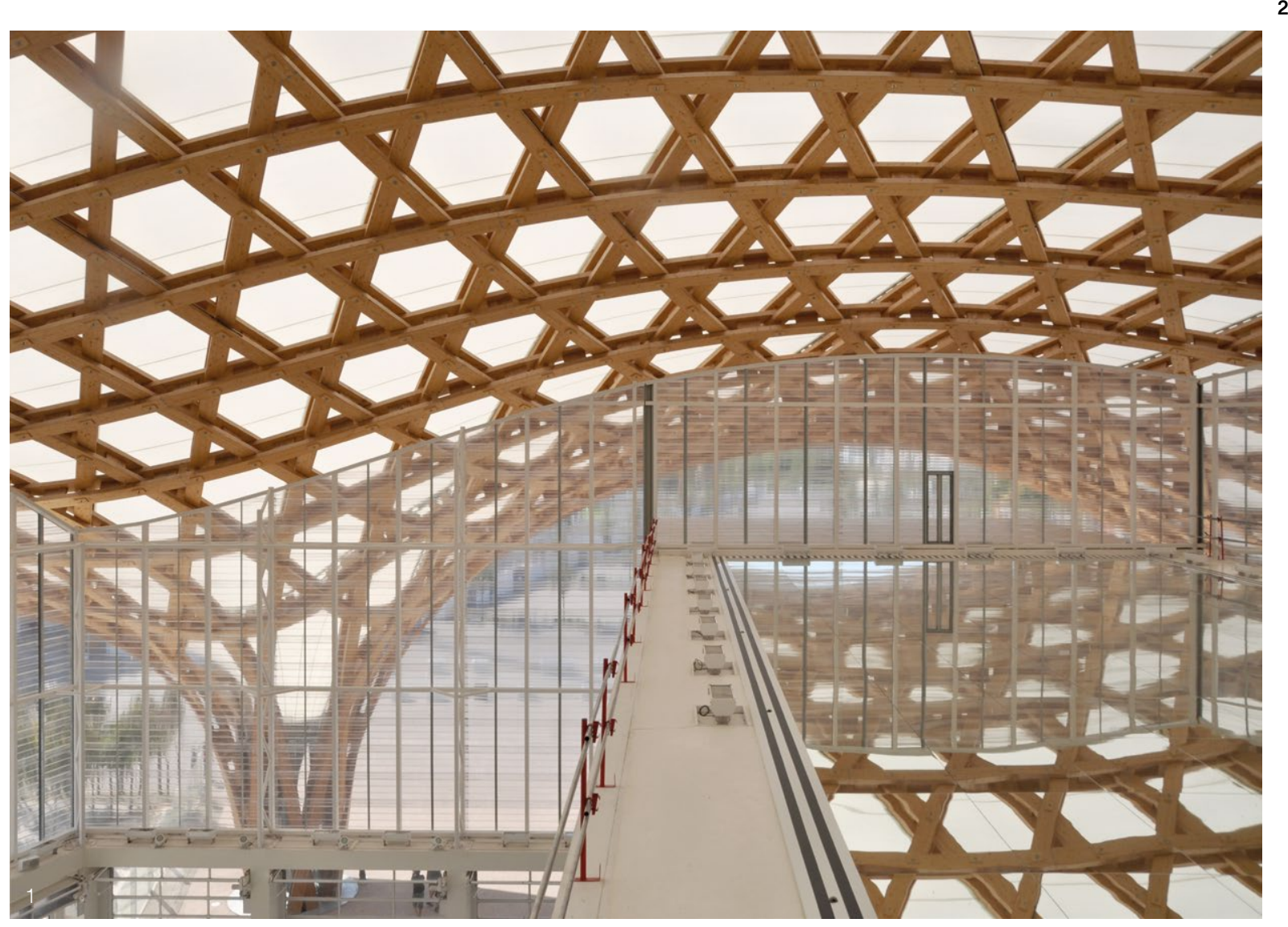

and professional developments. I took part in three colloquia, one in Copenhagen one in Vienna, and one in Berlin. In all colloquia these aspects arose.

AJ: Industrial cooperation with academia is al ways crucial, and I think mostly depends on personal relationships as well. How would you define the relevance of the academic-industry partnership?

MB: The ideal scenario for me is one we've created in Australia called the 'embedded practice' model and it has received Australian Research Counci funding. The participating architectural and ensineering practices had to funding. The participating architectural and engineering practices had to cover on thid of the costs and the government two third, which meant take the opportunity lightly.

The second thing that we did, which I think is really important for us, was to seek candidates who had already been working within practice for at least frive years, and in a situation where they had reached a plateau, a situation from where hey could see a massive loss of opportunity in the practice that they were in and an opportunity to make a difference. In other words, candidates who saw their PhD as a means to experiment on new ways of working within the practice.

Practice experience engenders knowledge on constraints that are

1. Centre Pompidou Metz. Architects: Sesign-to-Production and Blumer
Destication to that question is: how well did you get those things to happen? Industry or practice partners are mentors. Academics are supervisors. Each role must 


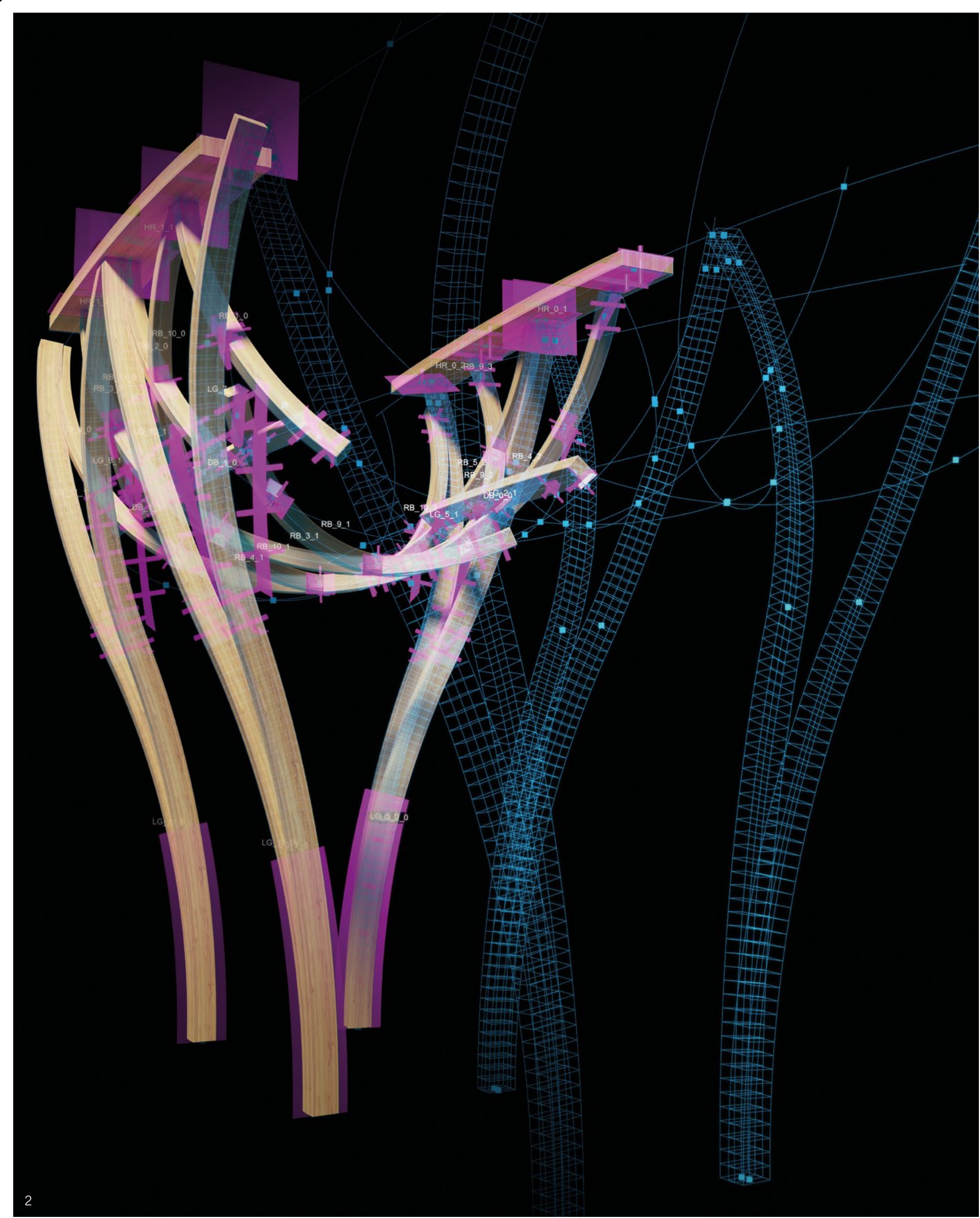

CG: Innochain has this configuration. The supervisors are from the academic you expressed it: ths from the side of the industry parters. But l see ll as you expressed it: the commitment of the industrial partner and their agenda must be clear. Looking back, I would say that smaller companies, with very to unexpected situations in the research praxis. In addition, they are highly to unexpected situations in the research praxis. In addition, they are highly
motivated to use and support the opportunity of in-house research and motivated to use and support the opportunity of in-house research and development, because normally they can't afford any investment in this kin of project on his scale. So, my recommendation would be to make such programmes more open and more attractive to small enterprises. This could be one of the findings of Innochain.

AJ: How would you define the urgency for collaborative design research with industry in the field of digital fabrication?

CG: For small-to-medium enterprises (SMEs) there's urgency because for them it's, as I said, very difficult to do or afford in-house research. Huge companies have better equipment and resources - sometimes better than small universities have, to be honest. Many large companies are also very tocused on selling established products. We don't have enough huge companies that invest in research for building processes. Too many are focused on selling materials and products. What we call the building industry today is mainly an industry of material production. There's only a very small amount of innovation going on at a large scale.

MB: We've had probably 30 years of insignificant productivity gains in the building industry compared with manufacturing, which is why it's a difficutt sector to find external funding from unless there's a climate of fear to milk, such as the digital isolation of practice at the turn of this century, when practices became aware that there were things they could be doing better, software that they could be accessing to give them competitive advantage, but they couldn't afford to take the risk to buy a full license. In this context, Innochain was perfect because candidates, embedded in practice as researchers, had access to cutting-edge technology and a network of peers in the same boat.

AJ: Do you think we can already identify the potential consequences of Innochain research?

MB: You'd have to do a matrix that charts what were the differences they were seeking to make, what they set out to do and what they achieved, once the PhDs are finished.

CG: Some industry partners are already quite happy. The Speckle project, for example, developed at UCL with Henn in Berlin is a successful case that got a tangible result, where the partner got a lot of interesting new ideas to improve their own work.

MB: So, what they would claim is that their moral high ground is that they facilitated a game-changing software environment? Innochain it wouldn't have happened.

CG: Yes, and the other point is, for the industry partners, I think they're using this because it was developed in dialogue with them. In Germany, I think we 

means there's only a very small number of architectural practices that are really trying to use

ad this asenda by funding afford ble housing and using this as a platform for innovation, because then you get everybody - engineers, architects, economists, builders and sociologists - all working together investigating how we transform fundamental operations. Such vital shifts in thinking and practice are not going to come from the market, that's for sure.

\section{MB: It's universal.}

CG: So for the partners it was a real boost for internal discussion: is this really productive, is this helpful? So, I think for them it's positive, and they wouldn't have had this level of access to the tool and to discussions around the tool if they hadn't been part of the development team.

MB: That's a very good point, when the researcher becomes an agent of change in the office in terms of their work and practice, it's a great outcome.

AJ: Innochain is aiming to establish a new paradigm for design research for construction, can this be achieved?

CG: I was struggling with this question because, in some contexts, we still have a huge discussion about what design research means. We have yet to understand the term.

MB. My answer is that design research is tautology, because if design doesn't have any research in it then you're not innovating, you're repeating or you're copying or you're mimicking. So you can't possibly design something as a response to an idea or question unless you do the research. What I would say from Innochain is that it was yet another fantastic opportunity to prove that design is research. There's three ways of looking at it as far as I'm aware.

You can do research about design and that's the stuff I find incredibly tedious - the researchers who sort of look at designers and seek to explain to everybody else what designers do, and they come up with terms like 'design thinking'. For me it's like 'music thinking' or 'surgery thinking'. Then there's design for research, which is like 'l'm going to design a new cup, so l'm going to look at cups in history and see what I need to know about cups and why we need new cups. But then there's design through research, which is "'m designing a cup and I'm treating that process as research. As I design the cup I'm going to reflect on what I'm doing and I'm going to use that reflection to improve not only the design of the cup that ''m undertaking but all my design processes and therefore my designs in the future'.

In this sense, PhDs are excellent opportunities for designers because hey are formalising the research aspect of design. Innochain really they are succeededing investigation and a new understanding is articulated in their thesis that would totherwise have been achieved if they hadn't the the work.

\section{AJ: Last question: If there's an Innochain 2, what should it address?}

CG: There's a real urgency for the building industry to reduce its $\mathrm{CO}^{2}$ emissions and consumption of resources. We are far too late with all this and we're part wo very conservative discipline. So for Innochain 2 or 2.0, whateveryou would call his, it should have a strong focus on the crucial contemporary challenges in the production of the built environment. One aim, for me, would be to focus on increasing diversity in construction methodo and in architectura design for specific local and regional contexts: 


\section{Biographies}

Zeynep Aksöz is a partner in the Vienna-based design research studio OpenFields. In her ongoing research, teaching and practice, Aksöz explores generative desig processes in the collaboration between human and machine intelligence, in particular, design as an emergent process, $\mathrm{co}$-authored by human and non-human agents. Aksöz was a Marie Curie fellow and Early Stage Researcher (ESR) in the Innochain Europe Training Network. As a result of this fellowship she has a technical doctorate from University of Applied Arts Vienna, that focuses on applications of $\mathrm{Al}$ and Machin Learning in the Early Design Phase.

Efilena Baseta is an architect and engineer whose interests lie in exploring material behaviours, both physically and digitally, in order to create innovative structures and optimise construction processes. She studied at the National Technical University of Athens the Institute for Advanced Architecture of Catalonia (IAAC) in Barcelona. In 2019, as an Innochain ESR, she received her technical doctorate with distinction from the University of Applied Arts Vienna. Baseta is an architect at Coop Himmelb(l)au; she is also a cofounding partner of Noumen - , she is also a cofounding partner of Noumena - a multidisciplinary practice that focuses on digitalising designs and sroduction methods, and merging computational strategles with advanced manufacturing techniques. construction exhibitions and has led vallous educa programmes inemationally. In 2014 she receducatio 'In 'Innovative Stction was aw Autodesk. 2019 mereceived a scholarsh in for the

Klaus Bollinger studied Civil Engineering at the Technical University Darmstadt and taught at Dortmund University. Since 1994 he has been Professor for Structural Engineering at the School of Architecture, University of Applied Arts Vienna, since 2000 he has been guest professor at the Stadelschule in Frankfurt. In $1983 \mathrm{Kl}$ Bollinger and Manfred Grohmann established the practice Bollinger + Grohmann, now located in Fran
am Main, Vienna, Paris, Oslo and Melbourne, with around 100 employees. The office provides a complete range of structural design services for clients and projects worldwide, and have collaborated successfully with numerous internationally recognised architects. The scope of their work includes building structures, façade design and building performance for commercial, retail and exhibition facilities as well as classic civil

engineering structures such as bridges, roofs and towers.

Charles Bouyssou is an architect and graduate of ENSA Paris - Malaquais (2015). He has worked for several years on architectural robotics and holds a strong IT background. Within XtreeE, Bouyssou focuses on the design, testing and commissioning of large-scale robotic systems for architectural applications.

Mads Brath Jensen is a PhD fellow at the Department of Architecture, Design and Media Technology at Aalborg University. Brath Jensen's research focus is on design methods and procedures, and how they can establish methods and procedures, and how they can establish a fabrication, with an emphasis on interactive, real-time human-material-robot processes. Brath Jensen teaches computational architecture, parametric design and rapid-prototyping technologies. His research-based and rapid-prototyping technologies. His research-based teaching has resulted in a series of pavilions showcasin findin in simulation, and fabrication techniques.

Johannes Braumann is professor for Creative Robotics at UfG Linz, leading an interdisciplinary team of researchers exploring robots as an interface between the digital and physical world. As co-founder of the Association for Robots in Architecture, Braumann is tightly linked with both the robotics and design communities. He is the main developer of the accessible robot simulation and programming too KUKAlprc, which is today used by more than 100 universities and 50 companies worldwide.

\section{Maite Bravo is an architect, educator and researcher} studying concepts and design methodologies
emerging from the use of digital design and its 
immersion into architectural praxis, with emphasis on robotic fabrication techniques and built shellstructures. She holds degrees from the University of Chile, IAAC, Universitat Politècnica de Catalunya, including a PhD with a 'Cum Laude' distinction from the latter. Her academic experience includes several universities including IAAC, $\mathrm{LCl}$, and $\mathrm{UIC}$ in Barcelona; Strathclyde; and ETH Zurich, among many others. She is currently senior faculty at the British Columbia Institute of Technology, Canada. Her professional experience includes several architectural firms in Chile, GBL Architects in Canada, Barcelona Municipality and Barcelona Regional Agency, B3 architects, and MuDD Architects. She has received several awards for her built and research work (including the prestigious MIT ACADIA 2017 research award, and a Dezeen Awards 'highly commended' mention), for ongoing collaborations with Stephanie Chaltiel on Robotic Fabrication for Monolithic Earthen Shells.

Giulio Brugnaro is an architect, designer and researcher working in the field of robotic fabrication for architectural production. He is currently a PhD candidate and Marie Curie Fellow at The Bartlett School of Architecture UCL as part of Innochain. His research focuses on developing adaptive robotic fabrication processes and sensing methods that designion to engage with materi behaviours and tool affordances to explore novel design opporturities. alfordances to exp a nerign Research Tutor on The Burtett's Design for Manufacture MA Trch programe.

\section{Stephanie Chaltiel is a French architect working with} digital fabrication and natural materials. She began her career in Mexico and French Guyana, building houses by hand and promoting local cultures. After working for Bernard Tschumi in New York on the International Center for the Americas, OMA for the Monaco Hotel and Zaha Hadid for the construction phase of Les Pierres Vives in Montpellier, she started her own practice, MuDD Architects, in 2019. Her award-Winning projects include Mud Shellin Lond and Terramia in Milan. They marry cutting-edge technology with raw materials and have been presented and exhibited worldwide, receiving the MIT ACADIA 2017 research award, a Dezeen Award 'highly commended' mention and being listed in the ICON Design 100 in 2019. Chaltiel has taught at SUTD Singapore, University of Westminster in London, The Architectural Association in London, Ravensbourne College in London, at the University of Brighton and more recently at Elisava Barcelona. As an Innochain Marie Curie ESR, she developed drone-spray

technology for sustainable architectures.

Angelos Chronis is head of the City Intelligence Lab at the Austrian Institute of Technology (AIT) in Vienna and an Innochain Marie Curie Fellow at IAAC in Barcelona. In addition, he has taught at IAAC, UCL, TU Graz and IUAV University of Venice. Chronis is the general chair of Symposium on Simulation for Architecture and Urban Design (SimAUD) and a member of its steering committee; he is actively involved in scientific committees internationally. He has worked across many fields including renerative design artificial intelligence, virtual and augmented reality and interactive installations. His main research interest lies in the integration of simulation, optimisation and in the drive in the design and fabrication process, with a focus on computational fluid dynamics.

Kenn Clausen is an architect and computational designer at GXN, the research and innovation unit at 3XN Architects. His research and interests range from parametric modelling and robotic fabrication to workflow trategies and geom per the team leading the digltal design group, working to fuse architecture and inovation by utllising a data-driven approach at all stages of projects. He works with applied research across scales, from large scale building projects to expernental prototypes. His current archlectural work includes the design and development of parametric models, geometry optimisation and work Hlow strategies for the Olympic House in Lausanne, the New Fish Market in Sydney and the SAP Garden sports arena in Munich. Denmark and UNStudio in Amsterdam.
Justin Dirrenberger is Associate Professor of Materials Science \& Engineering at Conservatoire National des Arts et Métiers in Paris. He holds a PhD in materials engineering from Ecole Nationale Superieure des Mines de Paris. His research is focused on architectural materials, additive manufacturing and multi-scale modelling. Dirrenberger contributes to various research and development programmes at XtreeE.

Moritz Dörstelmann is a registered architect and managing partner of FibR GmbH, a specialist company for computational design and robotic fabrication of bespoke fibre-composite structures, which enables the exploration of a novel design and construction repertoire for expressive high-performance lightweight structures. His work on digital fabrication technology provides socially relevant solutions for resource-efficient manufacturing and architectural construction, and explores integrative computational design methods as interface and catalyst for interdisciplinary collaboration. Dörstelmann developed the digital design and robotic fabrication strategies that underpin his work over seven years of research at the University of Stuttort years of research at the University of Stuttgart, Harverc Technologies at the Technical University of Munich.

Romain Duballet is a structural engineer, graduate of the Ecole Nationale des Ponts et Chaussés and ENSA Pais Malaquais. He has been working for several years on the Mation of topological He Hings ide his work at XtreeE, Duballet is currently pursuing a PhD in Hcole Nationale des Ponts et Chaussées and Ecole Nationale Supérieure des Arts et Métiers.

Al Fisher is a technical director and Head of Based in London, he helps orchestrate code

Based in London, he helps orchestrate code
development across a decentralised global network of contributors. Encouraging a diversity of skills and of contributors. Encouraging a diversity of skills and
experience - across engineering, computer science, experience - across engineering, computer science,
architecture, construction and design - the aim is to architecture, construction and design - the aim is to a globally accessible open coding framework. Fisher's background is in structural design computation, with a Master's and PhD in Civil and Architectural Engineering from the University of Bath. He has focused on performance-driven design and optimisation, applying these principles to projects such as the Louvre Abu Dhabi and the London Olympic Stadium Transformation. Most recently his research has focused on solving wider wicked-type problems and the subjective performance of co-authored distributed systems. He has a particular interest in the cultural and technological challenges of networked humanto-human and human-to-machine collaboration.

Gaudillière is an architect and a graduate of ENSA Paris - Malaquais (2016). She has been studying the environmental impact of additive manufacturing and its conditions of implementation in the contemporary construction context. She currently contributes to the supervision of XtreeE's prospective architectural and design projects.

Christoph Gengnagel studied structural engineering at the Bauhausuniversität Weimar and architecture at the Technical University of Munich; he has been working as a structural ensineer since 1993. He was a founding partner of the engineering office $k$ ingenieure in Munich and since 2013 has been a consulting partner with Bollinger + Grohmann Ingenieure. In 2005 he completed his PhD in Mobile Membrane Stuctures a the TU Munich. He was appointed as professor in the lepartment for structural design and engineering at Berlin Univer for stuctural design and engineering at the herld Arts a Arts, Copenhagen. Gengnagel's research focuses on computer-aided structural design and the development internationally.

Sean Hanna is Reader in Space and Adaptive Architectures at The Bartlett School of Architecture, and a member of the UCL Space Syntax Laboratory, ecognised as one of the UK's highest-performing research groups in the field of architecture and the built environment in consecutive UK Research Assessment 
Exercises. His research is primarily in developing computational methods for dealing with complexity in design and the built environment, including the comparative modelling of space, and the use of machine learning and optimisation techniques for the design and fabrication of structures. It is often conducted in close collaboration with leading architects, engineers, artist and technology producers. He has contributed to more than 100 academic publications addressing the fields of spatial modelling, machine intelligence, collaborative creativity, among others, and his work has been featured in the non-academic press, including The Architects' Journal and The Economis

Anja Jonkhans is an architect working and teaching at the University of Applied Arts in Vienna. She received her Part 2 diploma and Master's degree in architecture from The Bartlett School of Architecture, UCL. Having worked for, amongst others, Alsop Architects and Grimshaw Architects in London, she was foundin partner of Spacelab with Peter Cook, Colin Fournier and Niels Jonkhans for the Kunsthaus Graz Museum. Before teaching at the Angewandte she held a position as an assistant professor at the TU Graz between 2001 and 2004. Her teashing and research subjects 2001 and field of building construction and drafting techniques.

Ulrika Karlsson is an architect and founding member of the practices Brrum and Servo in Stockholm. She has a specific interest in the role of architectura She has a specific inter in the role of architectura representations and their transto information Korsson is Professor of Architectural and KTH School of Architecture Sto o k A chitecture at KTHSchool of Architecture, Stockholm and a guest prossor at Stchos at Konstfack, Stockholm, The Bartlet, UCL, and UCLA, Los Angeles. She received her Architecture degree from Columbia University, New York, and her Landscape Architecture degree from the Swedish University of Agricultural Sciences. She has contributed to numerous journals, curated exhibitions and exhibited internationally. KTH School of Architecture, through Karlsson, was an academic partner forl Inochain. In for the project Interiors Matter: A Live Interior, from the Swedish Research Council.

Jan Knippers is a structural engineer and since 2000 has been Head of the Institute for Building Structures and Structural Design (ITKE) at the University of Stuttgart. His interest is in innovative and resource-efficient structures at the intersection of research and development and practice. In 2001 Knippers co-founded Knippers Helbig Advanced Engineering, with offices in Stuttgart, Berlin and New York. Key projects are the EXPO Axis for Shanghai in 2010, the Thematic Pavilion at EXPO 2012 in Yeosu, South Korea, and the Gallery at the Staatsoper Berlin, in 2017. In 2018 he founded Jan Knippers Ingenieure, to give personal attention to innovative projects, from concept to completion From 2014 to 2019, Jan Knippers was coordinator of the collaborative research centre TRR141 Biological Design and Integrative Structures between the Universities of Stuttgart, Tübingen and Freiburg. Since 2019 he has been Deputy Director of the Cluster of Excellence in Integrative Computational Design and Construction, and Vice-Rector for Research of the University of Stuttgart.

Ayoub Lharchi is an architect and computational designer, interested in complex geometriestion designer, interested moomplex geometries and has worked internationally, from Germany to the USA, and is currently a PhD candidate and the USA, and is currently a PhD candidate and research associate, as part of Innochain, at the Centre for Inthrition Technology and Architecture (CITA), at the Royal Danish Academy of Fine Arts, Schools of Archis recture, Design and Conservation in Copenhagen. .

\section{Sean Lineham is a structural engineer at Arup's} Copenhagen office. Lineham's research focus is the implementation of technologies, tools and processes that will aid design and foster creativity. He has worked on projects internationally with Arup, from stadiums to art installations. Lineham's recent work includes Camp ark and the Japan Pavilion for Expo 2020 Dubai.
Alban Mallet is an architect and graduate of ENSA Paris - Malaquais (2015). He has been working for severa years on additive manufacturing processes and bio-sourced materials. At XtreeE, Mallet develops $3 \mathrm{D}$ printing processes for concrete, clay and polymers. He also contributes to the design of innovative largescale additive manufacturing systems.

Mathilde Marengo is an Australian-French-Italian architect whose research focuses on the contemporay urban phenomenon, its integration with technology, and its implications on the future of our planet. Within today's critical environmental, social and economic framework she investigates the responsibility of designers in answering these challenges through circular and metabolic design. She holds a PhD in Urbanism and is Head of Studies, faculty and PhD Supervisor in IAAC's Advanced Architecture Group, an interdisciplinary research group investigating emerging technologies of information, interaction and manufacturing for the design and transformation of the cities, buildings and public spaces. Working within the group's agenda of redefining the paradigm of design education in the information and experience age, Marengo carries research, design and experimentation with innovative eseacational formats based on holistic, multidisciplive educational formats bised on holistic, multidisciplinary materialistion, as well as through EU-funded projects including Innochain. Areti Markopoulou is a Greek architect, researcher and urban technologist working at the intersection A architecture and digttaltechnoles. She is the Academic Director at IAAC, where she also leads Advanced ArchitectureGroup. Her research and practice seek to redefine architecture as a performative "body 'beyond traditional notions of static materiality. approximate data, or standardised manufacturing. Markopoulou is co-founder of StudioP52 and co-editor of Urban Next, a global network focused on rethinking architecture through the contemporary urban milieu. She is the project coordinator of a number of funded European Research Projects on topics including urban egeneration though data science, circular design and construction and multidisciplinary educational models in the digital age. Markopoulou has also curated international exhibitions such as Future Arena and On Site Robotics (Building Barcelona Construma 2019 and 2017), Print Matter (In3dustry 2016), HyperCity (Shenzhen Bi-city Biennale, 2015) and MyVeryOwnCity (World Bank, BR Barcelona, 2011), while her work has been featured in exhibitions worldwide.

Morten Norman Lund has worked as a computationa esigner, architect and project manager at GXN since 2011, mainly working on research that focuses on material, sustainability and digital technology, as well as architectural projects. Norman Lund has been a part of GXN's digital fabrication project, Digital Factory, and is currently part of 3XN's work to integrate computational design research into the company's practice and workflows. Current architecture projects include the Cube, Berlin; Schüco Headquarters, Bielefeld; and Duale Hochschule Stuttgart.

Sivan Oesterle is an architect and researcher with special expertise in the field of computational design and digital fabrication. In 2010 he co-founded ROK an architectural office in Zurich. ROK's work focuses the development of integrated design processes at the intersection of design computation, ensineeringand digital fabrication. After graduating from ETH Zurich in 2007, he joined the Faculty of Architecture at ETHch in conducted research with Professors Gramazio \& Kohle. 2013 hewas a guest lecturer at the Acadeny o Fine. Ar Ar presents regulary at schools and conderences including the Architectural Association CITA and SmartGeluding Whilst at ETH Zurich, he received the Global Holm Whilstat ETHZunich, he received the Global Holcim

Anke Pasold is an associate professor at the Material Design Lab, Copenhagen School of Design and Technology, where she is developing the technologica side of the lab and structuring new educational programmes. Pasold's academic and research focus 
methodologies and fabrication processes by means of experimental prototypes. Educated as both an architect and a cross-disciplinary designer, Pasold is co-founder of the research-based architectural studio, AREA, in Copenhagen, with Isak Worre Foged. The studio explores academic activities, material properties, design methods, generative systems and fabrication techniques.

Paul Poinet is currently a Research Fellow in web-based collaboration at The Bartlett School of Construction and Project Management, and Design Tutor at The Bartlett School of Architecture, UCL. Poinet is working within a consortium of partners - Arup, Speckle Works, BuroHappold Engineering, 3D Repo, Rhomberg Sersa Rail Group, HOK and Atkins - on the Innovate-UK funded project AEC Delta Mobility, which aims to increase productivity, performance and quality in the construction industry by streamlining data exchange, in an open-source manner, early on in the design process. Previously, Poinet was an Innochain ESR and PhD Fellow at CITA, collaborating with Design-to-Production and BuroHappold Engineering.

Arthur Prior is a specialist in digital manufacturing. He received a Marie Skłodowska-Curie scholarship for research in additive manufacturing at $\mathrm{UCL}$ as part of esearch in a diventyarthor works a development ensineer for Staedtler Mars $\mathrm{GmbH}$ in Germany and also as a senior teaching fellow on The Germany and also as a seniorteaching fellow on The Bartelt's Design for Mn Previous Arth for Man facture MArch progrian for the Madrid-based company Factum Arte, renown for the international contemporary artists.

Line Rahbek is a senior architect at Dorte Mandrup Architects in Copenhagen. Rahbek specialises in complex modelling and works on the studio's inetrically advanced and large projects, mplementing Building Information Modelling (BIM) strategies and Information and Communications Technology (ICT) management. Over the past 14 including Zaha Hadid Architects, London; Gottlieb Paludan Architects, Copenhagen; and Cox Architecture, Melbourne, on projects including mixed-use high-rise buildings and rail transit. Rahbek has also taught at The Royal Danish Academy of Fine Arts, Copenhagen, and at RMIT University, Melbourne; and has been an invited guest critic at the Architectural Association and Centra Saint Martins in London.

Mette Ramsgaard Thomsen is Professor of Digital Technologies at CITA, which she founded in 2005 at the Royal Danish Academy of Fine Arts, Schools of Architecture, Design and Conservation. Her research examines how computation is changing the material cultures of architecture. By investigating advanced computer modelling, digital fabrication and material specification in projects including Predicitve Response, Complex Modelling and Innochain, she has been central in the forming of an international research field examining advanced modelling method that integrate simulation and predict material behaviour. In 2016 she was awarded the Elite Research Prize for outstanding researchers under 45 years of international excellence by the Danish Ministry for Higher Education and Science. In 2018 she was appointed Generand for the. UI 2023 world congress 'Sustainable Rut Christopher Robeller leads the Digital Timber Kaiserslautern Robeller previously werked of the Nation. Rober Fabrication Zurich IBOIS Labor in Research Digit Fabrication, Zurich, IBOIS Laboratory for Timber Constructions EPF, Lausaboratory the Timber of Computational Design (ICD) at the University of Stuttgart. Robeller's award-winning research on havative tmber structures, design for assembly and digtal fabrication is widely published and has been implemented in experimental structures, including the X-fix Timberdome pavilion in 2018. Recent buildings include the timber-folded plate structure for the Theatre Vidy-Lausanne
Philippe Roux is an engineer and graduate of Ecole and industrial engineering, as well as graduate of the University of New South Wales (Australia) in structural engineering, as well as ENSA Paris Malaquais (2016). Roux focuses on the optimisation of $3 \mathrm{D}$ printed concrete structures and on helping XtreeE clients to operate large-scale 3D printing systems.

onas Runberger is an architect, research and educator, ocusing on emergent technologies applied in practice. He heads Dsearch, and development team for computational design at White Arkitekter, where his team has been involved in over 80 Swedish and international architectural projects, as well as research projects. He is also Artistic Professor of Digital Design at Chalmers Department of Architecture and Civil Engineering in Gothenburg, heading the Architecture and Computation research group, and will be a research leader for architectural and structural design in the forthcoming Digital Twin Cities Research Center. Runberger was an industry representative in the Innochain project, with the ole of integrating the research of $\mathrm{PhD}$ candidates into role of integrating the research include the Royal Institute of Technology from which he also holds a PhD (2012) the Architecturam which School of Architecture and the ETH Zurich. Saman Saffarian is an architectural designer, technologist and researcher, and is currently the Associate Dean of Science and Research at the Faculty of Arts and Archtccture at the Technical University of Liberec. As part of the pursuing a PhD indigitally and materially-informed Strun at the Instifute for Bullding Structures and Structural Design (TKE), University of Stuttgart. His research focuses on design development and manufacturing of climate-adaptive bulling envelopes and the potential of material gradient fibre-reinforced polymer (FRP) for kinetic architectural applications. Previously, Saffarian worked as a lead designer for Zaha Hadid Architects in London, delivering concept design for projects and competitions of various scales and
Architects Computation and Design group (ZHAICODE), the development and fabrication of a number of experimental and researchbased installations and exhibitions.

Gavin Sayers is a structural engineer specialising in lightweight and tensile structures. He studied Civil Engineering at City University in London and subsequently gained an MBA from the University of Bath. He started his professional career with Tensys Ltd, developing in-house software and providing form-finding and patterning consulting services to the tensile industry. Upon leaving Tensys he continued as a specialist engineer at David Dexter Associates, helping to realise a portfolio of projects including stadia, tensile structures, aviaries and extensive ETFE projects worldwide. Sayers has recently joined AKTII as a Directo within their parametric applied research (p.Art) team where he continues to be fortunate enough to work alongside talented individuals in an exciting sector of the engineering field.

Fabian Scheurer is co-founder of Design-to-Production and leads the company's office in Zurich. He graduated from the Technical University of Munich with a diploma in computer science and architecture and gathered profession experience developer and new media consultant In 2002 he joined . Design (CAAD) group at the ETHZurich, where he De-founded Design-to-Production as a where he co founded Design-to-Production as a research group fabriction. At the nd fabrica comed up with architect Arnold Walz and became a enginmers and fabrcatos in the digita production of complex design.

Peter Scully is Technical Director of B-made,

The Bartlett Manufacturing and Design Exchange. He has worked in bespoke manufacturing for 25 years, developing a special focus on design at the interface between the disciplines, and has run companies that 
and artistic works. Scully has worked with architects, engineers and artists, deploying holistic project stakeholder understanding throughout the full process to curate workflows toward buildable outcomes. He has contributed to a range of built projects internationally, deploying procedural and tacit knowledge within design.

Bob Sheil is Professor in Architecture and Design through Production at The Bartlett School of Architec UCL, and has been Director of School since 2014. $\mathrm{He}$ is the author of multiple book chapters, refereed papers, and articles on design, making and technology. He has co-designed and built six artefacts/built works, and his work has been exhibited internationally on eleven occasions. He has edited seven books, including three issues of Architectural Design: Design through Making (2005), Protoarchitecture (2008), and High Definition: Negotiating Zero Tolerance (2014), an AD Reader, Manufacturing the Bespoke published in 2012. and 55/02: A sixteen*(makers) Monograph (also 2012). $\mathrm{He}$ is a Co-Founder of the FABRICATE conference and book for which he was $\mathrm{Co}-\mathrm{Chair}$ and $\mathrm{CO}$-Editor in 2011 (London) and 2017 (Stuttgart) and Co-Editor in 2020 (London).

Vasily Sitnikov is an architect and Innochain PhD researcher at KTH School of Architecture, Stockholm. Sitnikov's research concerns architectural tochnolm. sthecifically the advancement of precast concrete des, and production in consideration of contemporary environmental concerns and the development of dig fabrication. Sitnikov previously worked in a material fabication. Sitnkou previously worked hamaterial science laboratory in Moscow, where he honed his

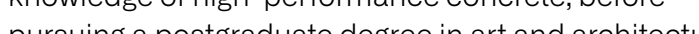
pursuing a postgraduate degree in art and architecture at Stadelschule, Frankfurtam Main. He then worked with Berlin-based artistand architect, Tomas Saraceno, on

Evy L. M. Slabbinck is a freelancer focusing on project development for Design-to-Production in Zurich. Until recently, she was a research associate and tutor
Design at the University of Stuttgart. Slabbinck gained her professional experience in various international practices, including Bollinger + Grohmann and Teuffe Engineering Consultancy, where she worked as a membrane engineer and computational specialist on several international projects. Her interests lie in structural and parametric design, form-finding and bending-active tensile structures. She has published and presented her work at international conferences and in journals, including the International Association for Shell and Spatial Structures (IASS) and the International Association for Bridge and Structural Engineering (IABSE). Slabbinck's PhD research, as part of Innochain, focuses on multiple states of equilibrium for bending-active (tensile) structures, in collaboration with Foster + Partners

James Solly is a Director at Format Engineers and a teaching fellow at The Bartlett School of Architecture and Architectural Design MEng programmes. James previously worked at Ramboll UK and BuroHappold Engineering before taking an academic role at the University of Stuttgart between 2015 and 2018. At the UTKE under Professor Jan Knippers, he was a member TTKE, under Professor Jan Knippers, he was a mem of the Innochain network: his research focused on the development of structural design methods for structures. He returned to London in fibre lattice structures. Hic returned to London in late 2018 where current professional roles.

Dimitrie Stefanescu is the founder of Speckle. He works as a senior software developer at Arup, Heworks as a senior soltware developer at Arup. where hever open source and proprietary the ro digtal design automation, data collection and computation globally across the firm and for Sp broader AEC industry, based on the open Speckle core. Previously, he was a Marie Curie Fellow at The Bartlett School of Architecture, as part of the Innochain network, whe hejniclue as partor the his research into digital design communication.
Hanno Stehling is partner and head of software development at the digital building process consultancy Design-to-Production in Zurich, where he leads a team of architects and programmers developing digital tools for CAD-CAM workflows and general data management for both in-house and external use. He graduated with a diploma in architecture from University of Kassel, Germany. He has a background in computer programming and gradually focused his studies onto the intersection between architecture and computer science, joining a research group about parametric design led by Prof. Manfred Grohmann of Bollinger + Grohmann and Oliver Tessmann (now Professor of Digital Design at TU Darmstadt). After graduating. he worked as a freelance programmer and as computational designer for renowned architects including Bernhard Franken, before joining Design-toProduction in 2009.

Kåre Stokholm Poulsgaard works with applied design research in architecture and is Head of Innovation GXN. He is interested in research and innovation in the built environment, specifically the way that digital technology impacts human wellbeing, work and learnin and what this means for design strategy. Alongside this, and what this means for design strategy. Alongside this, he is studying for a DPhil at the University of Oxford on how digtaltech nologies mpacthun and the organisation of work.

Kai Strehlke is working on the interface between digital data and CNC manufacturing of large-scale digtal data and $\mathrm{CNC}$ manufacturing of large-scale 2016 has been 2016 he has been teaching digital manufacturing at the Bern University of Applied Sciences. In 2015 he was invited by the Technical University Graz in Austria as a Guest Professor at the Institute of Architecture and Media. Between 2005 and 2015 he built up and led the Department of Digital Technologles at the architectural office Herzog \& de Meuron in Basel. He integrated a digital workshop in the office with different CNC technologies and with his team supported various projects on geometric to 2004 Strehlke researched and lectured at the chair of CAAD at the Swiss Federal School of Technology in Zurich and submitted his PhD with the theme of The Digital Ornament in Architecture, its Generation, Production and Application with Computer-

Controlled Technologies'.

Tom Svilans is an architectural designer and researcher based at CITA. His research as part of the Innochain network focuses on the use of emerging technologies to reveal new design potentials in freeform timber structures, and how this can lead to new practices formed around a deeper engagement with material behaviours. As part of this research, Svilans collaborates with Swiss timber contractor Blumer Lehmann AG and multidisciplinary architectural practice White Arkitekter AB. This research has been presented and published internationally in a variety of forums and peer-reviewed publications. As an educator, Svilans is a Senior

Teaching Fellow on the Design for Manufacture MArch programme at The Bartlett School of Architecture, UCL and runs fabrication-based student workshops at other institutes in the EU.

Martin Tamke is Associate Professor at CITA. He is pursuing design-led research into the interface and implications of computational design and its materialisation. He joined the newly-founded CITA in 2006 and shaped its design-based research practice. Projects on new is design-based research practice. Projects on new design and fabrication tools forwood fabrica practice engaged with bespoke moris and behaviour. . ils current work is characterised by strong 列 focus on Machine Learning and $3 \mathrm{D}$ sensing, structura engineering, with a focus on simulation and ult ralight hybrid structures, and material science, wh th a focus on bespoke computational knit. Currently he is involved in the European research projects Innochain and axskallerate, the Danish project Predictive Response 
Diederik Veenendaal is a structural engineer and parametric designer, working on lightweight structure and sustainable construction. He studied Civil Engineering at the University of Technology in Delft, and was a visiting student at the Louisiana State University (LSU) Hurricane Center, working on floatin structures and buoyant foundations. He completed his Master's thesis on the topic of evolutionary optimisation of fabric-formed beams. He started his professional career at Witteveen+Bos engineering consultants in the Netherlands, working on the groundfreezing of the downtown stations of the North/South subway line in Amsterdam and the structural design for the largest tensioned-membrane roof in the Netherlands, De Sches. He then joined the Block Research Group at the Institute of Technology in Architecture at ETH Zurich, Switzerland. While carrying out his doctoral research on flexibly formed concrete shell structures, he also co-edited the book Shell Structures for Architecture (Routledge, 2014), and coordinated the NEST HiLo project. He successfully defended his doctoral thesis in 2017 while also founding Summum Engineering in Rotterdam.

Valentin Viezens is a structural engineer. During and after his bachelor's degree, he worked in several engineering offices and was responsible for the planning of structures and construction details. He also worked as a teaching and constror structural anassis and eNurembers. In the laborato for stucturalengineering Nund building inforbotics, he gaist initial experience with experimental tests, 3D p measure solutions for constuctive and achitectural problem in solutions for constructive and paritectural problems in

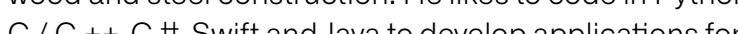
Wind $\mathrm{C}$, Swht and Java to develop applications for Windows, IOS and Android. He is currentlyworking as a research assistant on the development of plugins for DTC research group a

Helena Westerlind is an architect and $\mathrm{PhD}$ candidate in the technology research division at KTH School of Archichnogy research division at KTH School of strategies in additive manufacturing where she specifically addresses the relationship between robotic motion and material structures by applying principles of knitting in concrete deposition. As part of her interest in the history and prehistory of concrete, she is the initiator of the research project The Liquid Stone Cookbook, which traces entanglements between the human and the lithic from geological beginnings through historical recipes of artificial stone. She holds a degree in architecture from the Architectural Association, London and previously worked with Factum Arte in Madrid.

sak Worre Foged is an Associate Professor at Aalborg University, where he leads research on adaptive architecture. Worre Foged's primary research objective is the ongoing formulation of a theoretical, methodological and operational framework for environmental tectonics, approached through environmental morphogenetic design methods and models, and adaptive, environmentally-sensitive physical systems. Worre Foged is co-founder, with Anke Pasold, of AREA, Copenhagen.

Philip F. Yuan is Professor at the College of Architecture and Urban Planning (CAUP), Tongji University: Visiting Professor 2019 at Massachusetts Institute of Technology (MIT); and Thomas Jefferson Visiting Professor 2019 at University of Virginia. His research mainly focuse 2019 at field of performance-based tectonics in focuses on the fild of polotic fabrication. Yuan has published more well as robotic fabication. Yuan has published more weth English and Chisese His research and projects have

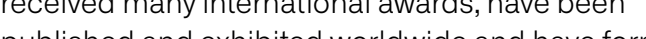
parts of several renoud museum collections, parts of severa renown

Mahriz Zakeri is an architect and graduate of ENSA Paris - Malaquais (2015). She also holds a bachelor of science . Engineering. AtXtreeE, Zakerifocuses on the

systems and on corresponding material science issues.

\section{Additional images}

Cover image: Digital production model of the MBridge prototype by Tom Svilans, Innochain Early-Stage Researcher and PhD Candidate, CITA.

p.2: Prototyping canopy components for the Elytra Filament Pavilion, developed in collaboration with James Solly. @ ICD/ITKE, University of Stuttgart. p.8: Stephanie Chaltiel presenting at the Innochain Project Mid-Review, at IAAC Valldaura Labs, 2017. Photo: Clara Orozco.

p.40: Innochain researchers Tom Svilans, Zeynep Aksöz and Dimitrie Stefanescu at Innochai 'Future Wood' workshop, Copenhagen, 2017. p.90: Efilena Baseta presenting at the Innochain Mid-Review, at IAAC valldaura Labs, 2017.

Photo: Clara Orozco.

p.142: Drone spraying by Stephanie Chaltiel. Photo captured by drone camera at Practice Futures - Building Design for a new Material Age exhibition, KADK, 2018

202. Hanif Kar of AKT II presenting at the Innochain Conference 2018, 'Expanding Information Modelling for a New Material Age'. Photo: Philip Ørneborg.

\section{Acknowledgements}

There are many people to thank for their help in putting this book together. from the EU commissioners who supported the Innochain grant application, to staff at each of the academic institutions and industry partner organisations who assisted in administering research development, events, content, communications, trave and accommodation. The complexities of this task were handled with unerring elegance, efficiency, cooperation, partnership and friendship by all. We are grateful to Lara Speicher and the team at UCL Press for their support and guidance. Finally, the editors wish to thank Laura Cherry, our project editor, for her immense dedication, patience, and care - qualities that have made this book all the more valuable and enjoyable to read and share. 
Design Transactions

Editors

Sheil, Mette Ramsgaard Thomsen

Project Editor

Design
Patrick M

Printing
Albe De Coker, Antwerp, Belgium

Copyeditors

Phebe Adler and Amanda Dale

First published in 2020 by

University College London

Gower Street
London WC1E 6BT

Available to download free: www.uclpress.co.uk

Text $\odot$ The Bartlett School of Architecture and the authors
Images $\odot$ The Bartlett School of Architecture and the authors

ACIP cota

A CIP catalogue record
The British Library.

This book is published under a Creative Commons 4.0 International license (CC BY 4.0). This license allows you to share, copy, distribute and transmit the work; to adapt the work and to make commercial use of the work providing atthbution is made to the authors (but not in any way that suggests that they endorse you or your use of
work). Atribution should include the following information:

Sheil, R.. Thomsen, M.R., Tamke, M., Hanna, S., (eds.). 2020. hestps://doi.org/10.14324/111.9781787355026

Further details about CC BY licenses are available at
http://Creativecommons.org/licenses/

fillicommons.org/licenses/

Any third-party material in this book is published under the book's Creative Commons license unless indicated otherwise in the credif line to the material. If you would like to reuse any third-party materia
not covered by the book's Creative Commons license you will neid to obtain permission directly from the copyright holder.

ISBN: 978-1-78735-489-0 (Hbk.)

DOl: https://doi.org/10.14324/111.9781787355026

${ }^{\star}$ UCLPRESS

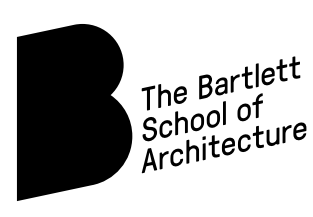

AIOCh

THE BARTLETT

The Innochain project received funding
from the European Union's Horizon 2020 on's Horizon 2020 under the Marie Skłodowska-Curie

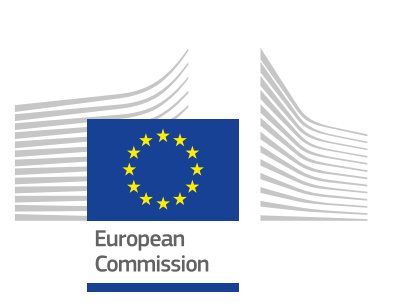

inrochrin
The Innochain Network (2015-2020)

was a shared research training

environment, examining how

advances in digital design tools

challenge building culture, enabling

sustainable, informed and materially

smart design solutions. The network

aimed to train a new generation of

interdisciplinary researchers with

a strong industry focus that can
effect real changes in the way

we think, design and build our

physical environment.

The Innochain project received

funding from the European Union's

Horizon 2020 research and innovation

programme under the Marie

Skłodowska-Curie grant agreement.

Early-Stage Researchers

Zeynep Aksöz

Efilena Baseta

Giulio Brugnaro

Stephanie Chaltiel

Angelos Chronis

Ayoub Lharchi

Arthur Prior

Saman Saffarian

Vasily Sitnikov

Evy Slabbinck

James Solly

Dimitrie Stefanescu

Tom Svilans

Helena Westerlind
Academic Institutions

ntre for Information Technology

and Architecture (CITA),

The Royal Danish Academy of

Fine Arts, Schools of Architecture,

Design and Conservation

(organising institution)
The Bartlett School of Architecture, UCL

Institute for Advanced Architecture of Catalonia (IAAC), Universitat Politècnica De Catalunya

IoA Institute of Architecture, The

University of Applied Arts, Vienna

Institute of Building Structures

and Structural Design (ITKE),

University of Stuttgart

KTH Royal Institute of Technology, Stockholm

Industry Partners

Bjarke Ingels Group (BIC)

Blumer-Lehmann AG

BuroHappold Engineering

Cloud 9 Architects

Design-to-Production

FibR GmbH

Foster + Partners

HENN Architecture

Robert McNeel \& Associates

ROK Architects

S-Form

Smith Innovation

stru.cture

White Arkitekter AB 


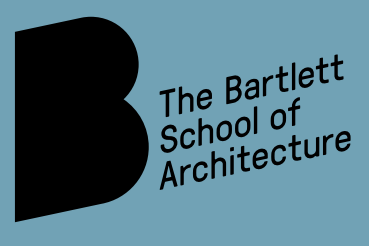

AIICII

THE BARTLETT

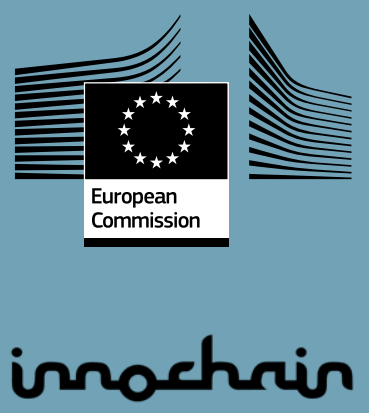

„UCLPRESS

Free open access versions available from www.ucl.ac.uk/ucl-press
Design Transactions: Rethinking Information Modelling for a New Material Age presents emerging research in design for architecture, engineering, and construction that challenges established building cultures and systems. Material is gathered from participants in Innochain, an EU-funded Horizon 2020 Training Network involving a consortium of six leading European architectural and engineering-focused institutions and their industry partners, and from participants in 'Expanding Information Modelling For A New Material Age', an international conference held at the Danish Architecture Centre in September 2018.

The book offers insight into new sustainable and materially smart design solutions with a strong focus on changing the way the industry thinks, designs, and builds our physical environment. Divided into sections exploring communication, simulation and materialisation, Design Transactions explores digital and physical prototyping and testing that challenges the traditional linear construction methods of incremental refinement. This novel research investigates the 'digital chain' between phases as an opportunity for extended interdisciplinary design collaboration. The book features work from all 15 early-stage researchers supported by Innochain, alongside chapters from world-leading industry collaborators and academics.

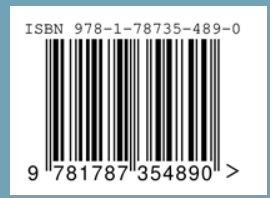

